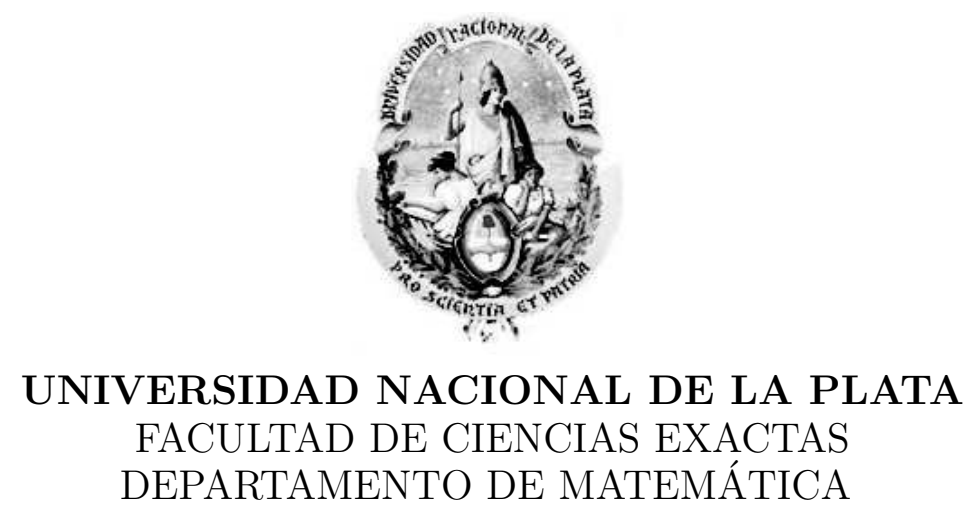

Tesis presentada para optar al título de Doctor en Ciencias de la Facultad de Cs. Exactas

\title{
PROBLEMAS DE APROXIMACIÓN EN ESPACIOS CON MÉTRICA INDEFINIDA
}

\author{
Francisco D. Martínez Pería
}

Directora: Dra. Alejandra L. Maestripieri

Codirector: Dr. Demetrio Stojanoff 

A mis amores, Belén y Malena. 



\section{Índice general}

1. Preliminares $\quad 27$

1.1. Teoría de operadores actuando sobre un espacio de Hilbert . . . . . . . . . . . . . 27

1.1.1. Factorización de Douglas y descomposición polar . . . . . . . . . . . . 27

1.1.2. Operadores densamente definidos . . . . . . . . . . . . . . . 31

1.1.3. Ángulos entre subespacios cerrados de un espacio de Hilbert . . . . . . . . 32

1.1.4. Inversas generalizadas . . . . . . . . . . . . . . . . . . . 34

1.2. Espacios vectoriales con métrica indefinida . . . . . . . . . . 36

1.2.1. Subespacios descomponibles . . . . . . . . . . . . . . . 38

1.2.2. Espacios de Krein y descomposiciones canónicas . . . . . . . . . . . . . . 40

1.2.3. Operadores sobre espacios de Krein . . . . . . . . . . . . . . . . . . . 43

1.2.4. Subespacios uniformemente definidos de un espacio de Krein . . . . . . . 46

1.2.5. Geometría en espacios de Krein . . . . . . . . . . . . . . . . . . . . . 49

2. Proyecciones autoadjuntas respecto a una métrica indefinida $\quad 55$

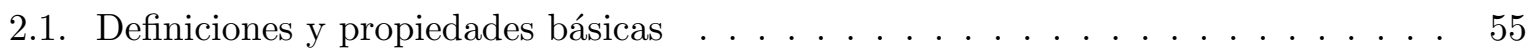

2.1.1. El operador de Gram inducido por un operador autoadjunto . . . . . . 58

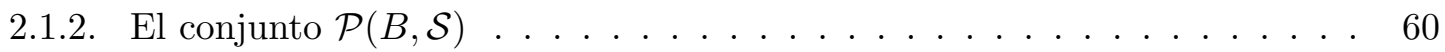

2.1.3. Representación matricial inducida por un subespacio compatible . . . . . 62

2.2. Condiciones necesarias y suficientes para la compatibilidad . . . . . . . . . 63

2.2.1. Descomposición de rangos de operadores . . . . . . . . . . . . . . . 65

2.2.2. Compatibilidad y ángulos entre subespacios . . . . . . . . . . . . 66

2.2.3. Proyecciones $B$-autoadjuntas para operadores de rango cerrado . . . . . . 68

2.3. Descomposición de proyecciones $B$-autoadjuntas $\ldots \ldots \ldots \ldots$. . . . 70

2.3.1. Proyecciones $B$-contractivas y $B$-expansivas . . . . . . . . . . . . . . 73

2.3.2. El fibrado de descomposiciones canónicas de un espacio de Krein . . . . . 75

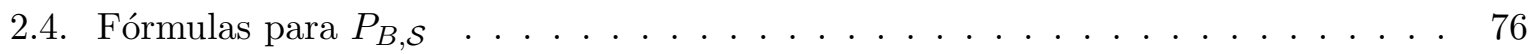

3. Complementos de Schur en espacios de Hilbert $\quad 83$

3.1. Complemento de Schur de operadores positivos . . . . . . . . . . . . . . 83

3.2. Complemento de Schur de operadores autoadjuntos . . . . . . . . . . . . . 87

3.2.1. Extremalidad del complemento de Schur a subespacios $B$-definidos . . . . 89

3.2.2. El complemento de Schur a una suma de subespacios . . . . . . . . . . . . 91

3.2.3. Una representación minimax respecto a subespacios $B$-indefinidos . . . . 93

3.3. Una fórmula para el complemento de Schur . . . . . . . . . . . . . . . . . 94 
4. Complementos de Schur en espacios de Krein

4.1. Propiedades extremales para subespacios $J$-definidos . . . . . . . . . . . . . . . . 101

4.2. Complementos de Schur de operadores $J$-positivos en espacios de Krein . . . . 105

5. Cuadrados mínimos indefinidos

5.1. El problema de cuadrados mínimos indefinidos . . . . . . . . . . . . . . 110

5.1.1. La ecuación normal . . . . . . . . . . . . . . . . . . . . 112

5.1.2. El conjunto de $B$-soluciones de cuadrados mínimos . . . . . . . . . . . . . 115

5.1.3. Minimizando en el conjunto de $B$-LSS de una ecuación lineal . . . . . . 116

5.2. Inversas generalizadas pesadas . . . . . . . . . . . . . . . 119

5.3. Una solución minimax para la ecuación $C x=y \ldots \ldots \ldots \ldots \ldots$ 


\section{Introducción}

La teoría de operadores lineales en espacios con métrica indefinida aparece por primera vez en el artículo "Hermitian operators in spaces with an indefinite metric" [62] de L. S. Pontryagin, publicado en el año 1944; si bien anteriormente algunos físicos teóricos se habían topado con estos espacios, este trabajo marca la aparición de una nueva rama del análisis funcional en la década de 1940. Cabe destacar que nos referimos a espacios de dimensión infinita, ya que las transformaciones lineales en espacios (de dimensión finita) con métrica indefinida han sido estudiadas desde fines del siglo XIX por G. F. Frobenius, entre otros.

El trabajo de Pontryagin fue continuado, principalmente, por M. G. Krein e I. S. Iokhvidov. Ellos axiomatizaron la teoría de espacios complejos con una métrica indefinida - a los que denominaron espacios $\Pi_{\kappa}$ - en base al enfoque de Pontryagin, considerando varios problemas referidos a la geometría de tales espacios y obteniendo una serie de resultados sobre los mismos. Además, Krein estudió los espacios $\Pi_{\kappa}$ en conexión con la transformada de Lorentz y la teoría de curvas en espacios de Lobachevskiy de dimensión infinita. Por su parte, Iokhvidov sugirió la aplicación de la transformada de Cayley-Neyman al estudio de las relaciones entre diferentes clases de operadores en $\Pi_{\kappa}$. Todo este material fue recopilado primero en la tesis de I. S. Iokhvidov y posteriormente en un extenso artículo escrito conjuntamente por Iokhvidov y Krein [44]. En 1959, los mismos autores publicaron una secuela de dicho artículo [45] incluyendo varias aplicaciones, entre ellas una al problema indefinido de momentos.

En esta época, la teoría comenzó a profundizarse y extenderse. En Finlandia, independientemente de los matemáticos soviéticos, R. Nevanlinna, E. Pesonen e I. S. Louhivaara comenzaron a estudiar problemas relacionados con una métrica indefinida. Poco tiempo después, el alemán G. Langer se sumó a este tipo de investigaciones, basándose tanto en los trabajos de los soviéticos como en los de los fineses.

En 1962 Yu. P. Ginzburg e Iokvidov publicaron, en forma conjunta, el primer tratado sobre geometría de espacios con métrica indefinida (de dimensión infinita) [35]. Este trabajo contenía resultados clásicos y, en parte, algunos de los resultados obtenidos a comienzos de los años ' 60 . En esta década se realizaron grandes avances para la teoría, descubriéndose nuevas aplicaciones, por ejemplo, a sistemas de ecuaciones diferenciales parabólicos e hiperbólicos disipativos (Philips), a oscilaciones de amplitud restringida en sistemas elásticos de dimensión infinita (Krein, Langer), a sistemas canónicos de ecuaciones diferenciales (Krein, Yakubovich, Derguzov), a la teoría de representación de grupos (Naymark), etc. Para el final de este período, J. Bognár [9] publicó el primer libro enteramente dedicado a espacios con métrica indefinida.

En los últimos años esta teoría ha sido redescubierta, provocando un nuevo y creciente interés en el desarrollo de estos temas. A continuación, describiremos los problemas que nos ocuparán en esta tesis. 


\section{Proyecciones autoadjuntas en espacios con métrica indefinida}

Desde los inicios de la teoría de operadores y el análisis matricial, las proyecciones han cumplido un papel principal en la solución de problemas de aproximación y optimización. Recientemente, las proyecciones han sido utilizadas como una herramienta central en las más variadas áreas de la matemática, la estadística y la ingeniería. Por ejemplo, y sólo por mencionar algunas, pueden enumerarse aplicaciones en procesamiento de señales, teoría de muestreo, wavelets, marcos, teoría de la información, métodos iterativos en álgebra lineal numérica, regresión lineal, ecuaciones integrales y computación paralela (en [20] puede encontrarse una extensa lista de trabajos sobre estas aplicaciones).

Dado un espacio de Hilbert $(\mathcal{H},\langle\rangle$,$) - complejo, separable y de dimensión infinita - sea$ $L(\mathcal{H})$ el álgebra de operadores lineales acotados sobre $\mathcal{H}$ y consideremos el conjunto $\mathcal{Q}$ formado por las proyecciones (lineales y acotadas) definidas sobre $\mathcal{H}$. Recordemos que, una proyección $Q \in \mathcal{Q}$ es un operador idempotente definido sobre $\mathcal{H}$, que proyecta los vectores de $\mathcal{H}$ sobre su rango $R(Q)$ a lo largo de su núcleo $N(Q)$, de acuerdo con la descomposición en suma directa

$$
\mathcal{H}=R(Q) \dot{+} N(Q) .
$$

De la teoría elemental de espacios de Hilbert sabemos que, dado un subespacio cerrado $\mathcal{S}$ de $\mathcal{H}$, para cada $y \in \mathcal{H}$ existe un único vector $x_{0} \in \mathcal{S}$ tal que $y-x_{0} \perp x$ para todo $x \in \mathcal{S}$, i.e. $y-x_{0} \in \mathcal{S}^{\perp}$. Luego, el operador $P_{\mathcal{S}} \in L(\mathcal{H})$ definido como $P_{\mathcal{S}}(y)=x_{0}$ resulta ser la (única) proyección autoadjunta sobre $\mathcal{S}$. También es común llamarla "la proyección ortogonal sobre $\mathcal{S}$ " ya que, en este caso, los subespacios $R\left(P_{\mathcal{S}}\right)=\mathcal{S}$ y $N\left(P_{\mathcal{S}}\right)=\mathcal{S}^{\perp}$ son ortogonales. Notemos que esta noción depende del concepto de ortogonalidad, y por consiguiente, del producto interno (o de la métrica indefinida) del espacio $\mathcal{H}$.

Ahora, dado un operador autoadjunto $B \in L(\mathcal{H})$, consideremos la métrica indefinida $\langle,\rangle_{B}$ inducida por $B$ :

$$
\langle x, y\rangle_{B}=\langle B x, y\rangle, \quad x, y \in \mathcal{H} .
$$

Tenemos entonces que $\mathcal{H}$, dotado con esta forma sesquilineal, resulta un espacio con métrica indefinida. En primer lugar, dado un subespacio cerrado $\mathcal{S}$ de $\mathcal{H}$, nos interesa caracterizar aquellas situaciones en las que existen proyecciones (con rango $\mathcal{S}$ ) que resulten $B$-autoadjuntas, es decir, autoadjuntas para la métrica indefinida $\langle,\rangle_{B}$. No es difícil comprobar que un operador $T \in L(\mathcal{H})$ es $B$-autoadjunto si y sólo si $B T=T^{*} B$, por lo que estudiaremos bajo qué condiciones el conjunto

$$
\mathcal{P}(B, \mathcal{S})=\left\{Q \in \mathcal{Q}: R(Q)=\mathcal{S}, B Q=Q^{*} B\right\}
$$

es no vacío; en dicho caso diremos que el $\operatorname{par}(B, \mathcal{S})$ es compatible. En particular, presentaremos condiciones necesarias y suficientes para la compatibilidad, parametrizaciones del conjunto $\mathcal{P}(B, \mathcal{S})$ y fórmulas para las proyecciones de este conjunto.

Este problema está motivado por una serie de trabajos de G. Corach, A. Maestripieri y D. Stojanoff, [19], [20], [21], en los que se considera la existencia de pares $(A, \mathcal{S})$ compatibles en el caso en que $A \in L(\mathcal{H})^{+}$, el cono de operadores semidefinidos positivos de $L(\mathcal{H})$.

A pesar de la similitud entre los planteos de ambos problemas, las técnicas utilizadas en cada caso son completamente diferentes. Los trabajos [19], [20] y [21] se basan en la geometría y la teoría de operadores sobre espacios de Hilbert, mientras que el problema que planteamos resolver necesitará de herramientas de la teoría de operadores sobre espacios con métrica indefinida. Para ilustrar estas diferencias, notemos que si $A \in L(\mathcal{H})^{+}$es inversible entonces existe una única 
proyección $A$-autoadjunta sobre $\mathcal{S}$, ya que $\langle,\rangle_{A}$ es un producto interno equivalente a $\langle$,$\rangle en$ el siguiente sentido: existen constantes $\alpha, \beta>0$ tales que

$$
\alpha\|x\| \leq\|x\|_{A} \leq \beta\|x\|, \quad \text { para todo } x \in \mathcal{H},
$$

siendo $\|x\|_{A}=\left\|A^{1 / 2} x\right\|=\langle A x, x\rangle^{1 / 2}$ la norma definida por el producto interno $\langle,\rangle_{A}$. Además, si $\mathcal{S}$ es un subespacio de dimensión finita, siempre existe una proyección $A$-autoadjunta sobre $\mathcal{S}$, aún cuando $A$ no es inversible (ver [19, Teorema 6.2]). Sin embargo, las afirmaciones anteriores dejan de ser ciertas en el caso indefinido, por ejemplo, si consideramos el espacio de Hilbert $\mathcal{H}=\mathbb{C}^{2}$, el subespacio $\mathcal{S}=\left\{(x, y) \in \mathbb{C}^{2}: y=-x\right\}$ y el operador autoadjunto

$$
J=\left(\begin{array}{cc}
1 & 0 \\
0 & -1
\end{array}\right)
$$

el par $(J, \mathcal{S})$ no es compatible. Notemos que en el ejemplo anterior el operador $J$ es una simetría, es decir, $J=J^{*}=J^{-1}$. En este caso, $\left(\mathcal{H},\langle,\rangle_{J}\right)$ es un espacio de Krein. La existencia de proyecciones autoadjuntas en estos espacios ha sido investigada en detalle y puede consultarse en la literatura clásica del tema (ver [9], [43], [4]).

Por otra parte, es bien sabido que una proyección $Q \in L(\mathcal{H})$ es ortogonal si y sólo si es una contracción, i.e.

$$
\langle Q x, x\rangle \leq\langle x, x\rangle, \quad \text { para todo } x \in \mathcal{H} .
$$

Este hecho puede generalizarse a proyecciones $A$-autoadjuntas, cuando $A$ es semidefinido positivo. En este caso, G. Corach et al. [19] probaron que una proyección $Q$ es $A$-autoadjunta si y sólo si es una $A$-contracción, i.e.

$$
\langle Q x, x\rangle_{A} \leq\langle x, x\rangle_{A}, \quad \text { para todo } x \in \mathcal{H},
$$

o equivalentemente, $Q^{*} A Q \leq A$.

En cambio, si alteramos el producto interno de $\mathcal{H}$ con un operador autoadjunto $B \in L(\mathcal{H})$, la afirmación anterior deja de ser cierta. De hecho, S. Hassi y K. Nordström [38] probaron que una proyección $Q \in L(\mathcal{H})$ es $B$-contractiva si y sólo si $Q$ es $B$-autoadjunta y $N(Q)$ es un subespacio $B$-no negativo de $\mathcal{H}$ (i. e. $\langle x, x\rangle_{B} \geq 0$ para todo $x \in N(Q)$ ). Es por este motivo que no sólo consideraremos proyecciones $B$-contractivas sino también proyecciones $B$-expansivas (aquellas que satisfacen $Q^{*} B Q \geq B$ ) y $B$-isométricas (las que cumplen $Q^{*} B Q=B$ ).

Notemos que estas son tres familias de proyecciones $B$-autoadjuntas, pero de ninguna manera forman una partición de dicho conjunto. Sin embargo, si $(B, \mathcal{S})$ es compatible, mostraremos que cualquier proyección $Q \in \mathcal{P}(B, \mathcal{S})$ puede factorizarse en términos de una proyección $B$ contractiva, una $B$-expansiva y una $B$-isométrica. Además, esta factorización es única si suponemos que los núcleos de estas proyecciones son dos a dos ortogonales.

\section{Complementos de Schur de operadores autoadjuntos}

El complemento de Schur de una matriz de bloques es un concepto clásico del análisis matricial. Aunque fue introducido formalmente por I. Schur [68] en 1917, existen evidencias de esta noción en trabajos realizados por J. J. Sylvester y P. S. Laplace a fines del siglo XIX. Supongamos dada una matriz $A$ de $(p+q) \times(p+q)$ de la forma

$$
A=\left(\begin{array}{ll}
a & b \\
c & d
\end{array}\right)
$$


donde $a, b, c, d$ son, respectivamente, matrices de $p \times p, p \times q, q \times p$ y $q \times q$. Si la matriz $a$ es invertible, el complemento de Schur de la matriz A (respecto al bloque a) es la matriz de $q \times q$ definida por

$$
A_{/ a}=d-c a^{-1} b
$$

El complemento de Schur aparece naturalmente como una posible generalización del método de eliminación gaussiano para resolver sistemas de ecuaciones lineales.

En 1974, D. Carlson, E. Haynsworth y T. Markham [13] propusieron una generalización del complemento de Schur para matrices arbitrarias: dada una matriz $A$ de $m \times n$ representada como en la Ec. (1) con $a, b, c$ y $d$, respectivamente, matrices de $k \times k, k \times(n-k),(m-k) \times k$ y $(m-k) \times(n-k)$, el complemento de Schur de la matriz $A$ (respecto al bloque $a$ ) es la matriz de $(m-k) \times(n-k)$ definida por

$$
A_{/ a}=d-c a^{\dagger} b,
$$

siendo $a^{\dagger}$ la inversa generalizada de Moore-Penrose de la matriz $a$.

Paralelamente, W. N. Anderson Jr. [2] descubrió una nueva manifestación del complemento de Schur. Dada una matriz (semidefinida) positiva $A$ de $(p+q) \times(p+q)$ escrita por bloques (considerando la descomposición ortogonal $\mathbb{C}^{p+q}=\mathbb{C}^{p} \oplus \mathbb{C}^{q}$ ) como en la Ec. (1), Anderson probó la existencia del máximo (respecto al orden usual entre matrices autoadjuntas) del conjunto

$$
\mathcal{M}\left(A, \mathbb{C}^{q}\right)=\left\{X \in \mathbb{C}^{p+q}: 0 \leq X \leq A, R(X) \subseteq \mathbb{C}^{q}\right\}
$$

al que denotó $A_{/ \mathbb{C}^{p}}$ y denominó "operador cortocircuito" de $A a \mathbb{C}^{p}$. La motivación para estudiar esta operación, así como también su nombre, provienen de la teoría de circuitos eléctricos: dado un circuito puramente resistivo de $n=p+q$ puertos, cuya matriz de impedancia $A$ es (semidefinida) positiva, si los primeros $p$ puertos (es decir, los correspondientes al bloque $a$ ) son cortocircuitados entonces el voltaje es forzado a permanecer en el subespacio ortogonal - ya que no puede haber voltaje a lo largo de un circuito en corto - y es fácil ver que la nueva matriz de impedancia es

$$
A_{/ \mathbb{C}^{p}}=\left(\begin{array}{cc}
0 & 0 \\
0 & c-b^{*} a^{\dagger} b
\end{array}\right)
$$

Anderson notó además que la matriz de impedancia es positiva sólo para circuitos resistivos. Para poder estudiar circuitos con componentes reactivos, es necesario extender la definición de operador cortocircuito a matrices que no sean necesariamente positivas.

Posteriormente, T. Ando [5] propuso una nueva generalización del complemento de Schur de matrices, ligada a la noción de operador cortocircuito. Si $A$ es una matriz compleja de $n \times n$ y $\mathcal{S}$ es un subespacio de $\mathbb{C}^{n}$, la matriz $A$ es $\mathcal{S}$-complementable si existen matrices $M_{r}$ y $M_{l}$ de $n \times n$ tales que

$$
P M_{r}=M_{r}, \quad M_{l} P=M_{l}, \quad P A M_{r}=P A \quad \text { y } \quad M_{l} A P=A P,
$$

siendo $P$ la proyección ortogonal sobre $\mathcal{S}$. Ando notó que la matriz $A M_{r}$ no depende de la elección de $M_{r}$ y $M_{l}$ y definió la compresión de Schur de $A$ a $\mathcal{S}$ como $A_{\mathcal{S}}=A M_{r}$ y el complemento de Schur de $A$ a $\mathcal{S}$ como $A_{/ \mathcal{S}}=A-A M_{r}$. En particular, si $A$ es semidefinida positiva y $\mathcal{S}$ es el subespacio generado por los primeros $k$ vectores de la base canónica de $\mathbb{C}^{n}$, la descomposición en bloques del complemento de Schur de $A$ a $\mathcal{S}$ es la dada en la Ec. (2), es decir, esta definición extiende la noción clásica de complemento de Schur. 
En 1975, Anderson y G. E. Trapp [3] extendieron la noción de operador cortocircuito (ó complemento de Schur) a espacios de Hilbert. Dados un operador $A \in L(\mathcal{H})^{+}$y un subespacio cerrado $\mathcal{S}$ del espacio de Hilbert $\mathcal{H}$, el complemento de Schur de $A$ a $\mathcal{S}$ es

$$
A_{/ \mathcal{S}}=\operatorname{máx}_{\leq}\left\{X \in L(\mathcal{H})^{+}: X \leq A, R(X) \subseteq \mathcal{S}^{\perp}\right\} .
$$

Vale destacar que la existencia del máximo (respecto al orden usual entre operadores autoadjuntos) del conjunto

$$
\mathcal{M}\left(A, \mathcal{S}^{\perp}\right)=\left\{X \in L(\mathcal{H})^{+}: X \leq A, R(X) \subseteq \mathcal{S}^{\perp}\right\}
$$

fue probada por M. G. Krein [51] en 1947, quien utilizó este operador distinguido en su teoría de extensión de operadores simétricos.

Notemos que la definición de complementabilidad propuesta por Ando puede aplicarse no sólo a matrices sino también a operadores (acotados) actuando entre espacios de Hilbert. Más aún, en [22] G. Corach et al. probaron que, dados un operador autoadjunto $B \in L(\mathcal{H})$ y un subespacio cerrado $\mathcal{S}$ de $\mathcal{H}$, el operador $B$ es $\mathcal{S}$-complementable si y sólo si el par $(B, \mathcal{S})$ es compatible. Además, en este caso resulta que

$$
B_{/ \mathcal{S}}=B(I-Q),
$$

siendo $Q$ cualquier proyección en $\mathcal{P}(B, \mathcal{S})$.

Por su parte, P. Massey y D. Stojanoff [54] probaron que, si $\mathcal{S}$ es un subespacio $B$-definido de $\mathcal{H}$, el complemento de Schur de $B$ a $\mathcal{S}$ puede caracterizarse como un extremo en un conjunto de operadores autoadjuntos similar al de la Ec. (3). Utilizando la descomposición de proyecciones $B$-autoadjuntas mencionada anteriormente, mostraremos que, dado un subespacio $B$-indefinido $\mathcal{S}$ de $\mathcal{H}$, el complemento de Schur de $B$ a $\mathcal{S}$ admite una representación del tipo minimax, es decir, el operador $B / \mathcal{S}$ es el mínimo entre el conjunto de máximos obtenidos al variar adecuadamente cierto conjunto de operadores.

Por otra parte, en 1992, E. L. Pekarev [59] probó la siguiente fórmula para el complemento de Schur de un operador $A \in L(\mathcal{H})^{+}:$si llamamos $\mathcal{M}$ a la clausura del subespacio $A^{1 / 2}(\mathcal{S})$ entonces

$$
A_{/ \mathcal{S}}=A^{1 / 2} P_{\mathcal{M}^{\perp}} A^{1 / 2},
$$

siendo $P_{\mathcal{M}^{\perp}}$ la proyección ortogonal sobre el subespacio $\mathcal{M}^{\perp}$.

Si $B \in L(\mathcal{H})$ es un operador autoadjunto y su descomposición polar es $B=J|B|$, con $|B| \in L(\mathcal{H})^{+}$y $J=J^{*}=J^{-1}$, suponiendo que el par $(B, \mathcal{S})$ es compatible probaremos que

$$
B / \mathcal{S}=J|B|^{1 / 2} P_{J(\mathcal{M}) \perp / / \mathcal{M}}|B|^{1 / 2},
$$

siendo $\mathcal{M}$ la clausura del subespacio $|B|^{1 / 2}(\mathcal{S})$. Es necesario aclarar en este punto que, aún cuando el par $(B, \mathcal{S})$ es compatible, la proyección $P_{J(\mathcal{M})^{\perp} / / \mathcal{M}}$ que aparece más arriba es un operador densamente definido. Sin embargo, en este caso la composición $P_{J(\mathcal{M})^{\perp} / / \mathcal{M}}|B|^{1 / 2}$ permanece acotada.

Además, mostraremos que si el par $(B, \mathcal{S})$ no es compatible pero la composición anterior define un operador acotado, la fórmula propuesta en la Ec. (5) puede utilizarse para extender la definición del complemento de Schur de operadores autoadjuntos, manteniendo las propiedades fundamentales del mismo. 


\section{Complementos de Schur en espacios de Krein}

Dado un espacio de Hilbert $(\mathcal{H},\langle\rangle$,$) , un operador J \in L(\mathcal{H})$ es una simetría si es unitario y autoadjunto, es decir, $J=J^{*}=J^{-1}$. Estos operadores merecen particular atención, ya que el espacio con métrica indefinida $\left(\mathcal{H},\langle,\rangle_{J}\right)$ asociado a una simetría $J \in L(\mathcal{H})$ resulta ser un espacio de Krein. Esta familia de espacios es la más pequeña que contiene a los espacios de Hilbert, a los antiespacios de espacios de Hilbert, y es cerrada al considerar sumas directas ortogonales. Por este motivo es, tal vez, la clase de espacios con métrica indefinida más profundamente estudiados.

En este contexto, el teorema de Bognár-Kramli [10] asegura que, si $A \in L(\mathcal{H})$ es un operador $J$-autoadjunto, entonces existe un espacio de Krein $\mathcal{K}$ y un operador inyectivo $D \in L(\mathcal{K}, \mathcal{H})$ tal que

$$
A=D D^{\#},
$$

donde $D^{\#} \in L(\mathcal{K}, \mathcal{H})$ denota al operador $J$-adjunto de $D$. Sin embargo, esta descomposición puede no ser única (para más detalles, ver los Preliminares o el trabajo de J. Rovnyak [65]). Diremos que un operador $J$-autoadjunto $A \in L(\mathcal{H})$ tiene la propiedad de factorización única (PFU) si, para cualquier par de descomposiciones $A=D_{i} D_{i}^{\#}$ con $D_{i} \in L\left(\mathcal{K}_{i}, \mathcal{H}\right)(i=1,2)$ como la mencionada anteriormente, existe un isomorfismo $U \in L\left(\mathcal{K}_{1}, \mathcal{K}_{2}\right)$ tal que $D_{1}=D_{2} U$.

Motivados por la fórmula (4) de Pekarev, proponemos la siguiente definición para el complemento de Schur de un operador $J$-autoadjunto actuando en un espacio de Krein: dado un operador $J$-autoadjunto $A$ (con PFU) actuando en un espacio de Krein $\mathcal{H}$ y un subespacio cerrado $\mathcal{S}$ de $\mathcal{H}$, definimos el complemento de Schur de $A$ a $\mathcal{S}$ como

$$
A_{/[\mathcal{S}]}=D P_{\mathcal{M}[\perp] / / \mathcal{M}} D^{\#},
$$

siendo $D \in L(\mathcal{K}, \mathcal{K})$ con $N(D)=\{0\}$ tal que $A=D D^{\#}$ y $\mathcal{M}$ la clausura del subespacio $D^{\#}(\mathcal{S})$ de $\mathcal{K}$. Es importante mencionar aquí que, para poder asegurar que la composición anterior define un operador acotado, debemos hacer una suposición adicional sobre el subespacio $\mathcal{M}$. De hecho, supondremos que $\mathcal{M}$ es un subespacio de Krein de $\mathcal{K}$, i.e. un subespacio tal que

$$
\mathcal{K}=\mathcal{M}+\mathcal{M}^{[\perp]},
$$

donde $\mathcal{M}^{[\perp]}$ representa al subespacio $J$-ortogonal a $\mathcal{M}$. Esta hipótesis nos permitirá además caracterizar el rango y el núcleo del complemento de Schur. También, si el subespacio $\mathcal{M}$ es $J$-definido, nos permitirá representar a $B /[\mathcal{S}]$ como el máximo (o mínimo) de un conjunto de operadores similar al de la Ec. (3).

Un caso particular, para el cual bien merece la pena definir y estudiar el complemento de Schur, es el conjunto de los operadores $J$-positivos sobre un espacio de Krein $\mathcal{H}$. Un operador $A \in L(\mathcal{H})$ se dice $J$-positivo si

$$
\langle J A x, x\rangle \geq 0 \quad \text { para todo } x \in \mathcal{H} \text {. }
$$

Esta familia de operadores cumple la PFU y, al igual que los operadores (semidefinido) positivos sobre un espacio de Hilbert, forman un cono. Notemos además que, si $A \in L(\mathcal{H})$ es un operador $J$ positivo (con descomposición de Bognár-Kramli $A=D D^{\#}$ ) y $\mathcal{S}$ es cualquier subespacio cerrado de $\mathcal{H}, \mathcal{M}$ es un subespacio $J$-no negativo de $\mathcal{K}$. Todo esto parece evidenciar entonces que ésta es la clase de operadores - entre aquellas donde puede definirse - en la cual el comportamiento del complemento de Schur está más proximamente emparentado con el del operador cortocircuito (de operadores positivos actuando en un espacio de Hilbert) definido por Anderson y Trapp. 


\section{Problemas de cuadrados mínimos indefinidos}

Dados dos espacios de Hilbert $\mathcal{H}$ y $\mathcal{K}$, un operador $C \in L(\mathcal{H}, \mathcal{K})$ y un vector $y \in \mathcal{K}$, sabemos que la ecuación $C x=y$ tiene solución si y sólo si $y \in R(C)$. En este caso, existe una única solución de la ecuación en el subespacio ortogonal al núcleo de $C$. Más aún, si $x_{0} \in N(C)^{\perp}$ es dicha solución, el conjunto de soluciones de la ecuación $C x=y$ es la variedad afín $x_{0}+N(C)$. Además,

$$
\left\|x_{0}\right\|=\min \{\|x\|: C x=y\},
$$

es decir, $x_{0}$ es la solución de $C x=y$ de norma mínima.

Más generalmente, si $y \in \mathcal{K}$ es un vector arbitrario, consideremos el problema de minimizar la magnitud $\|C x-y\|$ entre los vectores $x \in \mathcal{H}$, es decir, hallar los $u \in \mathcal{H}$ tales que

$$
\|C u-y\|=\min \{\|C x-y\|: x \in \mathcal{H}\} .
$$

Un elemento $u \in \mathcal{H}$ se dice solución de cuadrados mínimos (LSS, por "least squares solution") de la ecuación $C x=y$ si cumple con la identidad de la Ec (6).

Es fácil ver que $u$ es una LSS de $C x=y$ si y sólo si $C u=P_{\overline{R(C)}} y$, donde $P_{\overline{R(C)}}$ es la proyección ortogonal sobre la clausura de $R(C)$. Es decir, la ecuación $C x=y$ admite alguna solución de cuadrados mínimos si y sólo si $y \in R(C)+R(C)^{\perp}$. Por lo tanto, $C x=y$ tiene una LSS para todo $y \in \mathcal{K}$ si y sólo si $C$ tiene rango cerrado (para más detalles, ver la recopilación de M. Z. Nashed [56]).

Si $C \in L(\mathcal{H}, \mathcal{K})$ tiene rango cerrado, consideremos el conjunto $\mathcal{S}_{y}$ formado por las LSS de $C x=y$. Puede probarse que existe un único $u_{y} \in N(C)^{\perp}$ tal que $\mathcal{S}_{y}=u_{y}+N(C)$ y además

$$
\left\|u_{y}\right\|=\min \left\{\|u\|: u \in S_{y}\right\},
$$

es decir, $u_{y}$ es una LSS de $C x=y$ de norma mínima. Más aún, $u_{y}$ es el único elemento de $S_{y}$ con esta propiedad.

Por lo tanto, dado $C \in L(\mathcal{H}, \mathcal{K})$ de rango cerrado, puede definirse un operador $C^{\dagger}: \mathcal{K} \rightarrow \mathcal{H}$ mediante

$$
C^{\dagger} y=u_{y}, \quad y \in \mathcal{K},
$$

siendo $u_{y}$ la única LSS de $C x=y$ de norma mínima. En la década de 1920, E. H. Moore [55] probó que, dada una matriz $A$ (real o compleja), existe una única solución del sistema

$$
A X=P_{R(A)}, \quad X A=P_{R(X)} .
$$

Por su parte, en 1955 R. Penrose [61], independientemente del trabajo de Moore, demostró que dada una matriz $A$ (real o compleja) el siguiente sistema también admite única solución:

$$
A X A=A, \quad X A X=X, \quad(A X)^{*}=A X, \quad(X A)^{*}=X A .
$$

Sorprendentemente, la solución de los sistemas (7) y (8) coincide. De hecho, la solución es el operador $A^{\dagger}$ definido anteriormente, el cual se denomina inversa generalizada de Moore-Penrose de $A$. Además, Penrose dió varias caracterizaciones extremales relacionadas con $A^{\dagger}$, en particular probó que, dados $A \in \mathbb{R}^{m \times n}$ y $b \in \mathbb{R}^{m}$ - si \|\| es la norma euclídea - $A^{\dagger} b$ cumple:

$$
\left\|A\left(A^{\dagger} b\right)-b\right\|=\operatorname{mín}\left\{\|A x-b\|: x \in \mathbb{R}^{n}\right\}
$$


y es el (único) vector de norma euclídea mínima entre los que minimizan $\|A x-b\|$.

Notemos que los problemas planteados por Moore y Penrose pueden extrapolarse naturalmente a operadores acotados (de rango cerrado) actuando entre espacios de Hilbert, y la caracterización dada por la Ec. (9) se extiende naturalmente a dichos espacios.

El problema de calcular soluciones de cuadrados mínimos de una ecuación dada aparece naturalmente en problemas provenientes de la matemática aplicada ([15], [63], [64]), principalmente en espacios de dimensión finita. En algunos casos, dada una matriz $C \in \mathbb{C}^{m \times n}$ y un vector $y \in \mathbb{C}^{m}$, se plantea calcular la (o las) LSS de $C x=y$ (respecto a la norma inducida por una matriz positiva $A_{2} \in \mathbb{C}^{m \times m}$ ) que minimice la norma inducida por una matriz positiva $A_{1} \in \mathbb{C}^{n \times n}$, es decir, calcular $u_{0} \in \mathbb{C}^{n}$ tal que

$$
\left\|C u_{0}-y\right\|_{A_{2}} \leq\|C x-y\|_{A_{2}} \text { para todo } x \in \mathbb{C}^{n},
$$

$\left\|u_{0}\right\|_{A_{1}}=\operatorname{mín}\left\{\|u\|_{A_{1}}: u \in \mathbb{C}^{n}\right.$ satisface la Ec. (10) $\}$, siendo $\|w\|_{A_{i}}^{2}=w^{t} A_{i} w$, para $i=1,2$.

Para resolver dicho problema es necesario calcular una inversa generalizada pesada de $C$. Más precisamente, dadas $C \in \mathbb{C}^{m \times n}$ y $A_{1} \in \mathbb{C}^{n \times n}, A_{2} \in \mathbb{C}^{m \times m}$ definidas positivas, el sistema a resolver es

$$
C X C=C, \quad X C X=X, \quad\left(A_{2} C X\right)^{*}=A_{2} C X, \quad\left(A_{1} X C\right)^{*}=A_{1} X C .
$$

Mas aún, en algunos casos es necesario resolver una versión singular de este problema, es decir, $A_{1}$ y $A_{2}$ se suponen semidefinidas positivas. En este caso siempre existen soluciones de (11) y, a pesar de que en general hay una cantidad infinita de ellas, existe una única solución con norma euclídea mínima.

En otras aplicaciones, puede resultar beneficioso resolver el sistema (11) para operadores lineales acotados entre espacios de Hilbert. Es decir, dados dos espacios de Hilbert $\mathcal{H}$ y $\mathcal{K}$, un operador con rango cerrado $C \in L(\mathcal{H}, \mathcal{K})$ y operadores semidefinidos positivos $A_{1} \in L(\mathcal{H})^{+}$y $A_{2} \in L(\mathcal{K})^{+}$, consideremos la seminorma \|\|$_{A_{1}}$ en $\mathcal{H}$ definida por

$$
\|x\|_{A_{1}}=\left\langle A_{1} x, x\right\rangle^{1 / 2} \quad \text { para } x \in \mathcal{H}
$$

(resp. \|\|$_{A_{2}}$ en $\mathcal{K}$ definida por $\|x\|_{A_{2}}=\left\langle A_{2} x, x\right\rangle^{1 / 2}$, para $x \in \mathcal{K}$ ) y el sistema (11).

En [18], Corach y Maestripieri estudiaron este problema, presentaron condiciones necesarias y suficientes para la existencia de soluciones del sistema (11) y, en caso de que exista alguna, parametrizaron todas las soluciones de (11). En particular, demostraron que, dados $C \in L(\mathcal{H}, \mathcal{K})$ de rango cerrado, $A_{1} \in L(\mathcal{H})^{+}$y $A_{2} \in L(\mathcal{K})^{+}$existe una solución $D \in L(\mathcal{K}, \mathcal{H})$ del sistema (11) si y sólo si $\left(A_{1}, N(C)\right)$ y $\left(A_{2}, R(C)\right)$ son pares compatibles.

Además, suponiendo que los pares $\left(A_{1}, N(C)\right)$ y $\left(A_{2}, R(C)\right)$ son compatibles e $y \in \mathcal{K} \backslash R(C)$, probaron que $u_{0} \in \mathcal{H}$ satisface

$$
\left\|y-C u_{0}\right\|_{A_{2}} \leq\|y-C x\|_{A_{2}} \quad \text { para todo } x \in \mathcal{H}
$$

y $\left\|u_{0}\right\|_{A_{1}}=\operatorname{mín}\left\{\|u\|_{A_{1}}: u \in \mathcal{H}\right.$ satisface la Ec. (12) $\}$, si y sólo si existen $Q \in \mathcal{P}\left(A_{1}, N(C)\right)$ y $P \in \mathcal{P}\left(A_{2}, R(C)\right)$ tales que

$$
u_{0}=(I-Q) C^{\dagger} P y
$$

En los últimos años, diferentes problemas relacionados con métricas indefinidas han aparecido en aplicaciones tales como procesamiento de señales y control (ver [40], [41], [66], [67]). Motivadas 
por el creciente interés en encontrar estimadores que sean menos sensibles a inexactitudes de los datos o errores de medida, algunas técnicas de filtrado adaptativo y de control robusto (o $\mathcal{H}^{\infty}$ ) han sido formuladas como problemas de minimización de funcionales indefinidos. En estos casos, el producto interno usual $\langle x, y\rangle=y^{t} x$ del espacio euclídeo $\mathbb{R}^{m}$ es reemplazado por una forma sesquilineal indefinida de la forma $\langle x, y\rangle_{J}:=\langle J x, y\rangle=y^{t} J x$, donde

$$
J=\left(\begin{array}{cc}
I_{p} & 0 \\
0 & -I_{q}
\end{array}\right)
$$

es una simetría (o matriz de signatura), siendo $I_{p}$ e $I_{q}$ matrices identidad de dimensiones $p$ y $q$ respectivamente. Se considera entonces el problema de cuadrados mínimos indefinido: dados una matriz $A \in \mathbb{R}^{m \times n}$ con $m=p+q \geq n$ y un vector $b \in \mathbb{R}^{m}$, hallar $u \in \mathbb{R}^{n}$ tal que

$$
\langle J(A u-b), A u-b\rangle=\min _{x \in \mathbb{R}^{n}}\langle J(A x-b), A x-b\rangle .
$$

Contrariamente a los problemas de cuadrados mínimos clásicos, la aparición de la simetría $J$ (con inercia positiva y negativa) puede llevar a problemas de minimización que no necesariamente tengan solución.

Nuestro objetivo, en este caso, es considerar problemas similares a los estudiados en [18] pero para cuadrados mínimos indefinidos en lugar de cuadrados mínimos pesados.

\section{Organización de la tesis y descripción de los resultados originales}

A continuación detallaremos el contenido de esta tesis, remarcando cuales son los resultados originales incluídos en la misma.

El Capítulo 1 reúne resultados preliminares, los cuales consideramos necesarios para poder acceder a los resultados principales de este trabajo sin necesidad de consultar las fuentes originales. Los mismos están distribuidos en dos secciones, la primera contempla un breve repaso sobre algunos temas de teoría de operadores acotados (descomposición polar, factorización de operadores, ángulos entre subespacios, inversas generalizadas) y operadores densamente definidos sobre espacios de Hilbert. La segunda parte es una introducción a los espacios con métrica indefinida, poniendo particular énfasis en los espacios de Krein y su geometría.

El Capítulo 2 está dedicado a estudiar la existencia de proyecciones autoadjuntas en espacios con métrica indefinida. Dado un espacio de Hilbert $\mathcal{H}$ y un operador autoadjunto $B \in L(\mathcal{H})$, consideramos la métrica indefinida inducida por $B$ sobre $\mathcal{H}$, es decir,

$$
\langle x, y\rangle_{B}=\langle B x, y\rangle, \quad \text { para } x, y \in \mathcal{H} .
$$

La Sección 2.1 presenta las definiciones (subespacio $B$-ortogonal a un subespacio $\mathcal{S}$ dado, parte $B$ - isotrópica de $\mathcal{S}$, operador de Gram inducido por $B$, etc.) y propiedades básicas que utilizaremos para caracterizar a las proyecciones $B$-autoadjuntas, i. e. aquellas $Q \in \mathcal{Q}$ que satisfacen

$$
\langle Q x, y\rangle_{B}=\langle x, Q y\rangle_{B}, \quad \text { para todo } x, y \in \mathcal{H} .
$$

Recordemos que, dado un subespacio cerrado $\mathcal{S}$ de $\mathcal{H}$, decimos que el par $(B, \mathcal{S})$ es compatible si existe (al menos) una proyección $B$-autoadjunta con rango $\mathcal{S}$, i.e.

$$
\mathcal{P}(B, \mathcal{S})=\left\{Q \in L(\mathcal{H}): Q^{2}=Q, B Q=Q^{*} B, R(Q)=\mathcal{S}\right\} \quad \text { es no vacío. }
$$


Es importante remarcar aquí que $Q \in \mathcal{Q}$ es $B$-autoadjunta si y sólo si $R(Q)$ y $N(Q)$ son subespacios $B$-ortogonales. Luego, el par $(B, \mathcal{S})$ es compatible si y sólo si

$$
\mathcal{H}=R(Q)+N(Q) \subseteq \mathcal{S}+B(\mathcal{S})^{\perp} .
$$

Esta condición equivale a la existencia de un complemento algebraico del subespacio $\mathcal{S}$, pero no exige que éste sea único. Por ejemplo, si consideramos el subespacio $\mathcal{N}=\mathcal{S} \cap B(\mathcal{S})^{\perp}$, es fácil ver que $\mathcal{H}=\mathcal{S}+B(\mathcal{S})^{\perp}$ si y sólo si $\mathcal{H}$ se descompone en suma directa como:

$$
\mathcal{H}=\mathcal{S} \dot{+} B(\mathcal{S})^{\perp} \ominus \mathcal{N},
$$

siendo $B(\mathcal{S})^{\perp} \ominus \mathcal{N}=B(\mathcal{S})^{\perp} \cap \mathcal{N}^{\perp}$. Entonces, si $(B, \mathcal{S})$ es compatible, distinguiremos a la proyección oblicua

$$
P_{B, \mathcal{S}}=P_{\mathcal{S} / / B(\mathcal{S})^{\perp} \ominus \mathcal{N}}
$$

la cual es $B$-autoadjunta y tiene rango $\mathcal{S}$, es decir, $P_{B, \mathcal{S}} \in \mathcal{P}(B, \mathcal{S})$.

G. Corach et al. [19], [20], [22], [24] estudiaron este problema en el caso en que $B$ es un operador positivo, es decir, cuando $\langle,\rangle_{B}$ es una forma sesquilineal semidefinida. La Sección 2.1 está conformada por resultados de dichos artículos o por versiones un poco más generales de los presentados allí, sin embargo los argumentos utilizados para demostrar estas últimas son esencialmente los mismos que en caso en que el operador es positivo.

En la Sección 2.2 estableceremos dos clases de condiciones necesarias y suficientes para asegurar la compatibilidad de un par $(B, \mathcal{S})$.

En primer lugar, probaremos que la compatibilidad de $(B, \mathcal{S})$ es equivalente a que ciertos rangos de operadores puedan descomponerse "ortogonalmente". En [21], G. Corach et al. mostraron que, dado un operador $A \in L(\mathcal{H})^{+}$y un subespacio cerrado $\mathcal{S}$ de $\mathcal{H}$, el par $(A, \mathcal{S})$ es compatible si y sólo si $A^{1 / 2}(\mathcal{S})$ es un subespacio cerrado en $R\left(A^{1 / 2}\right)$ y el rango de $A^{1 / 2}$ admite una descomposición ortogonal en términos del subespacio $\mathcal{M}=\overline{A^{1 / 2}(\mathcal{S})}$ :

$$
R\left(A^{1 / 2}\right)=\mathcal{M} \cap R\left(A^{1 / 2}\right) \oplus \mathcal{M}^{\perp} \cap R\left(A^{1 / 2}\right) .
$$

Dado un operador autoadjunto $B \in L(\mathcal{H})^{s}$, cuya descomposición polar está dada por $B=J A$ con $A \in L(\mathcal{H})^{+}$y $J=J^{*}=J^{-1}$, considerando una vez más el subespacio $\mathcal{M}=\overline{A^{1 / 2}(\mathcal{S})}$, mostraremos que el par $(B, \mathcal{S})$ es compatible si y sólo si $A^{1 / 2}(\mathcal{S})=\mathcal{M} \cap R\left(A^{1 / 2}\right)$ y

$$
R\left(A^{1 / 2}\right)=\mathcal{M} \cap R\left(A^{1 / 2}\right) \dot{+} J(\mathcal{M})^{\perp} \cap R\left(A^{1 / 2}\right) .
$$

Es importante remarcar que, en esta descomposición de $R\left(A^{1 / 2}\right)$, los subespacios intervinientes no son ortogonales respecto al producto interno del espacio de Hilbert $\mathcal{H}$, sino que son $J$ ortogonales en el espacio de Krein que queda determinado al considerar al operador $J$ como simetría fundamental sobre $\mathcal{H}$.

Posteriormente, mostraremos que la compatibilidad del par $(B, \mathcal{S})$ también puede interpretarse en términos del ángulo entre ciertos subespacios cerrados, es decir, el par $(B, \mathcal{S})$ es compatible si y sólo si el ángulo entre ciertos subespacios no es nulo. Más precisamente, $(B, \mathcal{S})$ es compatible si y sólo si

$$
c_{0}\left(\mathcal{S}^{\perp}, \overline{B(\mathcal{S})}\right)<1 .
$$

Finalmente, estudiaremos la existencia de proyecciones $B$-autoadjuntas para un operador $B$ de rango cerrado. En este caso, si $B=J A$ es la descomposición polar de $B\left(\operatorname{con} A \in L(\mathcal{H})^{+}\right.$y 
$\left.J=J^{*}=J^{-1}\right), R\left(A^{1 / 2}\right)$ también es un subespacio cerrado de $\mathcal{H}$ y, como $N\left(A^{1 / 2}\right)=N(B) \subseteq$ $B^{-1}\left(\mathcal{S}^{\perp}\right)=B(\mathcal{S})^{\perp}$, probaremos que $(B, \mathcal{S})$ es compatible si y sólo si $\mathcal{M}=A^{1 / 2}(\mathcal{S})$ es cerrado y

$$
\mathcal{H}=\mathcal{M}+J(\mathcal{M})^{\perp} .
$$

Notemos que esta última ecuación asegura la existencia de una (única) proyección $J$-autoadjunta con rango $\mathcal{M}$. Por lo tanto, si $B$ tiene rango cerrado, podemos dar una caracterización completa de la compatibilidad del par $(B, \mathcal{S})$ en términos del subespacio $\mathcal{M}$. De hecho, probaremos que las siguientes condiciones son equivalentes:

1. $(B, \mathcal{S})$ es compatible.

2. $P_{\mathcal{M} / / J(\mathcal{M})^{\perp}} \in L(\mathcal{H})$.

3. $\mathcal{M}$ es cerrado y $c_{0}\left(\mathcal{M}, J(\mathcal{M})^{\perp}\right)<1$.

4. $\mathcal{M}$ es un espacio de Krein de $\mathcal{H}$ (con la métrica indefinida inducida por $J$ ).

Es natural preguntarse sobre la posibilidad de extender este resultado al caso en que $B$ es un operador autoadjunto cualquiera. Sin embargo, presentaremos un ejemplo de un par $(B, \mathcal{S})$ no compatible para el cual el par asociado $(J, \mathcal{M})$ es compatible.

La sección 2.3 está dedicada al estudio de posibles descomposiciones y factorizaciones de las proyecciones $B$-autoadjuntas.

En primer lugar, recordemos que, dada una proyección $Q \in L(\mathcal{H})$ actuando en un espacio de Hilbert $\mathcal{H}$, las siguientes condiciones son equivalentes:

1. $Q$ es autoadjunta, i.e. $Q$ es una proyección ortogonal,

2. $Q$ es un operador positivo, i.e. $\langle Q x, x\rangle \geq 0$ para todo $x \in \mathcal{H}$,

3. $Q$ es una contracción, i.e. $\langle Q x, Q x\rangle \leq\langle x, x\rangle$ para todo $x \in \mathcal{H}$.

En cambio, S. Hassi y K. Nordström [38] mostraron que, cuando consideramos la métrica indefinida $\langle,\rangle_{B}$ inducida por un operador autoadjunto $B \in L(\mathcal{H})$, una proyección $B$-autoadjunta $Q \in L(\mathcal{H})$ es $B$-positiva (resp. $B$-contractiva) si y sólo si su rango $R(Q)$ (resp. su núcleo $N(Q)$ ) es un subespacio $B$-no negativo de $\mathcal{H}$. Recordemos que un operador $T \in L(\mathcal{H})$ es $B$-positivo (resp. $B$-contractivo) si $B T \in L(\mathcal{H})^{+}$(resp. $\left.T^{*} B T \leq B\right)$, o equivalentemente,

$$
\langle T x, x\rangle_{B} \geq 0 \text { para todo } x \in \mathcal{H} \quad\left(\text { resp. }\langle T x, T x\rangle_{B} \leq\langle x, x\rangle_{B} \text { para todo } x \in \mathcal{H}\right) \text {. }
$$

Dado un subespacio arbitrario $\mathcal{S}$ de $\mathcal{H}$ consideremos su parte $B$-isotrópica $\mathcal{N}=\mathcal{S} \cap B(\mathcal{S})^{\perp}$. Luego, podemos descomponer a $\mathcal{S}$ en suma directa de la siguiente manera:

$$
\mathcal{S}=\mathcal{N} \dot{+} \ominus \mathcal{N}
$$

siendo $\mathcal{S} \ominus \mathcal{N}=\mathcal{S} \cap \mathcal{N}^{\perp}$. Además, es fácil ver que el complemento $\mathcal{S} \ominus \mathcal{N}$ es un subespacio $B$-no degenerado (o sea, su parte $B$-isotrópica es trivial). Esta descomposición plantea naturalmente interrogantes acerca de la posibilidad de descomponer al subespacio $\mathcal{S} \ominus \mathcal{N}$, si éste fuera indefinido, en suma directa de un subespacio $B$-positivo y otro $B$-negativo. El siguiente es el resultado principal de esta sección, el mismo responde afirmativamente a la pregunta anterior (en el caso en que $(B, \mathcal{S} \ominus \mathcal{N})$ es un par compatible): 
Teorema (Existencia de descomposición). Dados $\mathcal{S}$ un subespacio cerrado de $\mathcal{H}$ y $B \in L(\mathcal{H})^{s}$, supongamos que $\mathcal{N}=\mathcal{S} \cap N(B)$. Luego, $(B, \mathcal{S})$ es compatible si y sólo si existe una descomposición de $\mathcal{S} \ominus \mathcal{N}$ como $\mathcal{S} \ominus \mathcal{N}=\mathcal{S}_{+}+\mathcal{S}_{-}$, siendo $\mathcal{S}_{+}$un subespacio (cerrado) B-no negativo, $\mathcal{S}_{-}$ un subespacio (cerrado) B-no positivo, $\left(B, \mathcal{S}_{ \pm}\right)$es compatible y $\mathcal{S}_{+} \perp_{B} \mathcal{S}_{-}$.

Además, entre todas las posibles descomposiciones dadas por el teorema, existe una única que satisface además que $\mathcal{S}_{+}$es un subespacio $B$-positivo, $\mathcal{S}_{-}$es un subespacio $B$-negativo y los subespacios $\mathcal{S}_{+}$y $\mathcal{S}_{-}$son ortogonales respecto al producto interno de $\mathcal{H}$.

Notemos que, si $(B, \mathcal{S} \ominus \mathcal{N})$ es compatible entonces $(B, \mathcal{S})$ es compatible y, además, cada $Q \in \mathcal{P}(B, \mathcal{S})$ puede escribirse como

$$
Q=P_{B, \mathcal{S} \ominus \mathcal{N}}+P_{\mathcal{N} / /(\mathcal{S} \ominus \mathcal{N}+N(Q))}
$$

Mas aún, por el Teorema anterior, sabemos que existen un subespacio $B$-no negativo $\mathcal{S}_{+}$y un subespacio $B$-no positivo $\mathcal{S}_{-}$tales que $\mathcal{S} \ominus \mathcal{N}=\mathcal{S}_{+} \dot{+} \mathcal{S}_{-},\left(B, \mathcal{S}_{ \pm}\right)$es compatible y $\mathcal{S}_{+} \perp_{B} \mathcal{S}_{-}$. Luego, es fácil ver que $P_{B, \mathcal{S} \ominus \mathcal{N}}=Q_{+}+Q_{-}$y, por lo tanto:

$$
Q=Q_{+}+Q_{-}+P_{\mathcal{N} / /(\mathcal{S} \ominus \mathcal{N}+N(Q))},
$$

siendo $Q_{+}$una proyección $B$-positiva, $Q_{-}$una proyección $B$-negativa y $P_{\mathcal{N} / /(\mathcal{S} \ominus \mathcal{N}+N(Q))}$ una proyección $B$-neutra. Es decir, cada $Q \in \mathcal{P}(B, \mathcal{S})$ admite una descomposición $Q=Q_{0}+Q_{+}+Q_{-}$ tal que $Q_{0} \in \mathcal{Q}$ es $B$-neutra, $Q_{+} \in \mathcal{Q}$ es $B$-positiva y $Q_{-} \in \mathcal{Q}$ es $B$-negativa.

Finalmente, mostraremos que toda proyección $B$-autoadjunta $Q \in L(\mathcal{H})$ admite una factorización $Q=E_{0} E_{+} E_{-}$, siendo $E_{0}, E_{+}$y $E_{-}$proyecciones en $L(\mathcal{H})$ que conmutan entre sí y tales que $E_{+}$es $B$-contractiva, $E_{-}$es $B$-expansiva y $E_{0}$ es $B$-isométrica (i.e. $\left\langle E_{0} x, E_{0} x\right\rangle_{B}=\langle x, x\rangle_{B}$ para todo $x \in \mathcal{H})$.

En la Sección 2.4, dado un par compatible $(B, \mathcal{S})$, presentaremos una serie de fórmulas para la proyección distinguida $P_{B, \mathcal{S}}$. Comenzemos estudiando qué ocurre si $B$ es inversible y, posteriormente, intentaremos generalizar la fórmula obtenida a un contexto más amplio.

Supongamos $B \in L(\mathcal{H})$ es autoadjunto e inversible y consideremos su descomposición polar $B=J A$, con $A \in L(\mathcal{H})^{+}$y $J=J^{*}=J^{-1}$. Como $A \in L(\mathcal{H})^{+}$también es inversible, la forma sesquilineal $\langle,\rangle_{A}$ es un producto interno en $\mathcal{H}$ equivalente al producto interno original $\langle$,$\rangle . Además, como J$ conmuta con $A$, tenemos que $J$ también es una simetría con respecto al producto interno $\langle,\rangle_{A}$ y, por lo tanto, $\left(\mathcal{H},\langle,\rangle_{A}\right)$ también es un espacio de Krein con simetría fundamental $J$.

Recordemos que, si $B$ es un operador de rango cerrado, la compatibilidad del par $(B, \mathcal{S})$ es equivalente a que la (única) proyección $J$-ortogonal con rango $\mathcal{M}=A^{1 / 2}(\mathcal{S}), P_{\mathcal{M} / / J(\mathcal{M})^{\perp}}$, sea acotada. Luego,

$$
P_{B, \mathcal{S}}=A^{-1 / 2} P_{\mathcal{M} / / J(\mathcal{M})^{\perp}} A^{1 / 2} .
$$

Consideremos ahora un operador autoadjunto $B \in L(\mathcal{H})$ no necesariamente inversible y, si $\mathcal{S}$ es un subespacio cerrado de $\mathcal{H}$, consideremos el subespacio cerrado $\mathcal{M}=\overline{A^{1 / 2}(\mathcal{S})}$.

Como ya mencionamos anteriormente, en general no podemos asegurar que la proyección $P_{\mathcal{M} / / J(\mathcal{M})^{\perp}}$ sea un operador acotado. Sin embargo, si $(B, \mathcal{S})$ es compatible, $P_{\mathcal{M} / / J(\mathcal{M})^{\perp}}$ tiene dominio denso (el subespacio $\mathcal{M}+J(\mathcal{M})^{\perp}$ ) y probaremos que el producto

$$
P_{\mathcal{M} / / J(\mathcal{M})^{\perp}} A^{1 / 2}
$$


permanece acotado, $R\left(P_{\mathcal{M} / / J(\mathcal{M}) \perp} A^{1 / 2}\right) \subseteq A^{1 / 2}(\mathcal{S})$ y además:

$$
P_{\mathcal{M} / / J(\mathcal{M})^{\perp}} A^{1 / 2}=A^{1 / 2} P_{B, \mathcal{S}} .
$$

Las fórmulas que presentaremos en esta sección son consecuencia de la Ec. (19), y obtendremos las mismas a partir de inversas generalizadas (o aún inversas densamente definidas) del operador $A^{1 / 2}$.

Los resultados de las Secciones 2.2, 2.3 y 2.4 forman parte del artículo "Decomposition of Selfadjoint Projections in Krein Spaces" [53] publicado en la revista Acta Scientiarum Mathematicarum en el año 2006.

El Capítulo 3 está dividido en dos partes, la primera (Sección 3.1) es una recopilación de resultados sobre el complemento de Schur de operadores positivos (actuando en un espacio de Hilbert), y la segunda (Secciones 3.2 y 3.3) contiene la definición del complemento de Schur de operadores autoadjuntos y los resultados originales que desarrollaremos acerca de este tema.

Como hemos mencionado anteriormente, si $A \in L(\mathcal{H})^{+}$el complemento de Schur de $A$ a un subespacio $\mathcal{S}$ de $\mathcal{H}$ puede caracterizarse como

$$
A_{/ \mathcal{S}}=\inf _{\leq}\left\{Q^{*} A Q: Q \in \mathcal{Q}, N(Q)=\mathcal{S}\right\},
$$

considerando el orden usual en $L(\mathcal{H})$ inducido por $L(\mathcal{H})^{+}$(ver [3]). Más aún, P. Massey y D. Stojanoff [54] probaron que, dados un operador autoadjunto $B \in L(\mathcal{H})$ y un subespacio (cerrado) $B$-no negativo $\mathcal{S}$ de $\mathcal{H}$, si el par $(B, \mathcal{S})$ es compatible entonces

$$
B / \mathcal{S}=\min _{\leq}\left\{Q^{*} B Q: Q \in \mathcal{Q}, N(Q)=\mathcal{S}\right\}
$$

Análogamente, si $\mathcal{S}$ es un subespacio $B$-no positivo de $\mathcal{H}$ y el par $(B, \mathcal{S})$ es compatible, $B_{/ \mathcal{S}}=$ máx $\leq\left\{Q^{*} B Q: Q \in \mathcal{Q}, N(Q)=\mathcal{S}\right\}$.

En la Sección 3.2 mostraremos que si $\mathcal{S}$ es un subespacio $B$-indefinido de $\mathcal{H}$ y el par $(B, \mathcal{S})$ es compatible, el complemento de Schur de $B$ a $\mathcal{S}$ admite una representación minimax. Para esto, necesitaremos probar dos propiedades del complemento de Schur:

1. $B_{/ \mathcal{S}}$ no depende de la parte $B$-isotrópica del subespacio $\mathcal{S}$. Más precisamente, probaremos que $B_{/ \mathcal{S} \ominus \mathcal{N}}=B_{/ \mathcal{T}}=B_{/ \mathcal{S}}$ para todo subespacio cerrado $\mathcal{T}$ de $\mathcal{H}$ tal que

$$
\mathcal{S} \ominus \mathcal{N} \subseteq \mathcal{T} \subseteq \mathcal{S}+N(B)
$$

2. El complemento de Schur a una suma de subespacios está relacionados con el complemento de Schur a cada uno de ellos. Específicamente, dados dos subespacios cerrados $\mathcal{S}_{1}$ y $\mathcal{S}_{2}$ de $\mathcal{H}$, tales que $\mathcal{S}_{1} \perp_{B} \mathcal{S}_{2}$ y $\mathcal{S}_{1} \cap \mathcal{S}_{2}=\{0\}$, si $\mathcal{S}_{1} \dot{+} \mathcal{S}_{2}$ es cerrado, mostraremos que

$$
B / \mathcal{S}_{1} \dot{+} \mathcal{S}_{2}=\left(B / \mathcal{S}_{1}\right) / \mathcal{S}_{2}=\left(B / \mathcal{S}_{2}\right) / \mathcal{S}_{1} .
$$

Luego, obtendremos una representación del complemento de Schur $B_{/ \mathcal{S}}$ para un subespacio cerrado $B$-indefinido $\mathcal{S}$, a partir de la descomposición $\mathcal{S} \ominus \mathcal{N}=\mathcal{S}_{+} \dot{+} \mathcal{S}_{-}$presentada en el Capítulo 2. De hecho, mostraremos que, si $(B, \mathcal{S})$ es compatible entonces

$$
B_{/ \mathcal{S}}=\left(B / \mathcal{S}_{+}\right) / \mathcal{S}_{-}=\left(B_{/ \mathcal{S}_{-}}\right) / \mathcal{S}_{+},
$$

donde $\mathcal{S} \ominus \mathcal{N}=\mathcal{S}_{+} \oplus \mathcal{S}_{-}$es la descomposición dada en el Teorema 2.3.2. Finalmente, utilizando la expresión de $B / \mathcal{S}$ obtenida en la Ec. (21), probaremos el siguiente resultado: 
Teorema. Sean $B \in L(\mathcal{H})^{s}$ y $\mathcal{S}$ un subespacio cerrado de $\mathcal{H}$. Si $(B, \mathcal{S})$ es compatible entonces

$$
B / \mathcal{S}=\operatorname{mín}_{N\left(Q_{+}\right)=\mathcal{S}_{+}} \operatorname{máx}_{N\left(Q_{-}\right)=\mathcal{S}_{-}} Q_{+}^{*}\left(Q_{-}^{*} B Q_{-}\right) Q_{+}=\operatorname{máx}_{N\left(Q_{-}\right)=\mathcal{S}_{-}} \operatorname{mín}_{N\left(Q_{+}\right)=\mathcal{S}_{+}} Q_{-}^{*}\left(Q_{+}^{*} B Q_{+}\right) Q_{-},
$$

donde $\mathcal{S} \ominus \mathcal{N}=\mathcal{S}_{+} \oplus \mathcal{S}_{-}$es la descomposición dada en el Teorema 2.3.2.

Una interesante consecuencia de este teorema es que, si el par $(B, \mathcal{S})$ es compatible entonces, fijado un vector $x \in \mathcal{H}$, tenemos que

$$
\langle B / \mathcal{S} x, x\rangle=\min _{s \in \mathcal{S}_{+}} \operatorname{máx}_{t \in \mathcal{S}_{-}}\langle B(x-(s+t)), x-(s+t)\rangle .
$$

Esta representación se asemeja a la obtenida en [3] por Anderson y Trapp para el complemento de Schur de un operador (semidefinido) positivo.

En la Sección 3.3 mostraremos que, dados un operador autoadjunto $B \in L(\mathcal{H})$ y un subespacio cerrado $\mathcal{S}$ de $\mathcal{H}$ tales que $(B, \mathcal{S})$ es compatible, hay una generalización natural de la fórmula de Pekarev (4) para el complemento de Schur de $B$ a $\mathcal{S}$ : si $B=J A$ es la descomposición polar de $B$, con $A \in L(\mathcal{H})^{+}$y $J=J^{*}=J^{-1}$, el complemento de Schur de $B$ a $\mathcal{S}$ puede calcularse como

$$
B_{/ \mathcal{S}}=J A^{1 / 2} P_{J(\mathcal{M}) \perp / / \mathcal{M}^{A}} A^{1 / 2}
$$

siendo $\mathcal{M}=\overline{A^{1 / 2}(\mathcal{S})}$.

Más aún, mostraremos que, si la fórmula (23) define un operador acotado, la misma puede utilizarse para extender la definición de $B_{/ \mathcal{S}}$ en casos en los que el par $(B, \mathcal{S})$ no es compatible, manteniendo las propiedades básicas del complemento de Schur. De hecho, si suponemos que $B / \mathcal{S}$ está definido por la Ec. (23), probaremos que

1. $B / \mathcal{S}$ es un operador autoadjunto;

2. $R(B) \cap \mathcal{S}^{\perp} \subseteq R\left(B_{/ \mathcal{S}}\right) \subseteq R\left(A^{1 / 2}\right) \cap \mathcal{S}^{\perp}$;

3. $N\left(B_{/ \mathcal{S}}\right)=A^{-1 / 2}(\mathcal{M})$.

Si $A \in L(\mathcal{H})^{+}$y $\mathcal{S}$ un subespacio cerrado de $\mathcal{H}$, el rango y el núcleo del complemento de Schur de $A$ a $\mathcal{S}$ fue estudiado originalmente en [3], [51] y [59]. Suponiendo que el par $(A, \mathcal{S})$ es compatible, G. Corach et al. [20] obtuvieron una descripción más precisa de los mismos: $R\left(A_{/ \mathcal{S}}\right)=R(A) \cap \mathcal{S}^{\perp}$ y $N\left(A_{/ \mathcal{S}}\right)=N(A)+\mathcal{S}$. Además, estas caracterizaciones del rango y el núcleo de $A_{/ \mathcal{S}}$ y $A_{\mathcal{S}}$ se alcanzan si y sólo si el par $(A, \mathcal{S})$ es compatible.

El resultado de Corach et al. puede generalizarse a operadores autoadjuntos. Si $B \in L(\mathcal{H})$ es autoadjunto y la fórmula (23) define un operador $B_{/ \mathcal{S}}$ acotado, probaremos que las siguientes condiciones son equivalentes:

1. $(B, \mathcal{S})$ es compatible,

2. $R\left(B_{/ \mathcal{S}}\right)=\mathcal{S}^{\perp} \cap R(B)$ y $N\left(B_{/ \mathcal{S}}\right)=\mathcal{S}+N(B)$.

Los resultados de las Secciones 3.2 y 3.3 fueron publicados a principios de este año en Linear Algebra and its Applications, con el título "Shorting selfadjoint operators in Hilbert spaces" [36]. 
En el Capítulo 4 introduciremos el concepto de complemento de Schur para operadores $J$ autoadjuntos en espacios de Krein.

Dado un espacio de Hilbert $\mathcal{H}$, E. L. Pekarev propuso definir el complemento de Schur de un operador $A \in L(\mathcal{H})^{+}$a un subespacio (cerrado) $\mathcal{S}$ de $\mathcal{H}$, mediante la fórmula

$$
A_{/ \mathcal{S}}=A^{1 / 2} P_{\mathcal{M}^{\perp}} A^{1 / 2}
$$

donde $P_{\mathcal{M}^{\perp}}$ es la proyección ortogonal con núcleo $\mathcal{M}=\overline{A^{1 / 2}(\mathcal{S})}$.

Si $\mathcal{H}$ es un espacio de Krein con simetría fundamental $J_{\mathcal{H}}$, dado un operador $J$-autoadjunto $A \in L(\mathcal{H})$, el Teorema de Bognár-Kramli asegura que existe un espacio de Krein (con simetría fundamental $\left.J_{\mathcal{K}}\right)$ y un operador inyectivo $D \in L(\mathcal{K}, \mathcal{H})$ tal que

$$
A=D D^{\#}
$$

siendo $D^{\#}$ el operador $J$-adjunto de $D$. Luego, si suponemos que el subespacio $\mathcal{M}=\overline{D^{\#}(\mathcal{S})}$ es

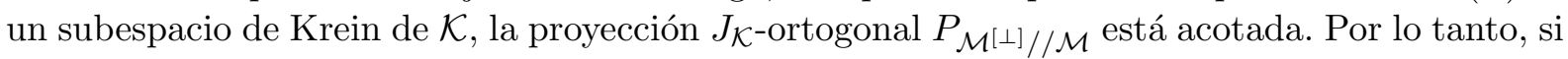
$A \in L(\mathcal{H})$ es un operador con la propiedad de factorización única (PFU), el siguiente operador está bien definido y es acotado:

$$
A_{/[\mathcal{S}]}=D P_{\mathcal{M}[\perp] / / \mathcal{M}} D^{\#}
$$

Si llamamos complemento de Schur de $A$ a $\mathcal{S}$ a este operador, probaremos que $A_{/[\mathcal{S}]}$ es un operador $J_{\mathcal{H}}$-autoadjunto y además:

$$
R(A) \cap \mathcal{S}^{[\perp]} \subseteq R\left(A_{/[\mathcal{S}]}\right) \subseteq R(D) \cap \mathcal{S}^{[\perp]} \quad \text { y } \quad N\left(A_{/[\mathcal{S}]}\right)=\left(D^{\#}\right)^{-1}(\mathcal{M}) .
$$

Estas caracterizaciones se asemejan a las obtenidas en el Capítulo 3 para el complemento de Schur de un operador autoadjunto actuando en un espacio de Hilbert y, al igual que en dicho caso, mostraremos que las inclusiones mencionadas más arriba pueden ser estrictas.

Posteriormente, estudiaremos las posibles caracterizaciones extremales de $A_{/[\mathcal{S}]}$ en aquellos

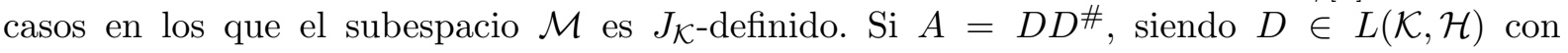
$N(D)=\{0\}$ y $\mathcal{K}$ un espacio de Krein, consideremos el conjunto

$$
\mathcal{I}(A)=\left\{X=E E^{\#}: E \in L(\mathcal{K}, \mathcal{H}), R(E) \subseteq R(D)\right\} .
$$

Si $A$ tiene la PFU el subespacio $R(D)$ sólo depende del operador $A$ y, en consecuencia, lo mismo ocurre con el conjunto $\mathcal{I}(A)$. Luego, podemos enunciar los resultados principales de la Sección 4.1 de la siguiente manera:

Teorema. Dados un operador $J$-autoadjunto $A \in L(\mathcal{H})$ con la PFU y un subespacio cerrado $\mathcal{S}$ de $\mathcal{H}$, supongamos que el subespacio $\mathcal{M}=\overline{D^{\#}(\mathcal{S})}$ es un subespacio de Krein de $\mathcal{K}$. Luego,

1. si $\mathcal{M}$ es $J$-no negativo entonces $A_{/[\mathcal{S}]}=\operatorname{máx}_{\leq_{J}}\left\{X \in \mathcal{I}(A): X \leq_{J} A, R(X) \subseteq \mathcal{S}^{[\perp]}\right\}$.

2. si $\mathcal{M}$ es $J$-no positivo entonces $A_{/[\mathcal{S}]}=\min _{\leq_{J}}\left\{X \in \mathcal{I}(A): A \leq_{J} X, R(X) \subseteq \mathcal{S}^{[\perp]}\right\}$. 
Teorema. Sea $\mathcal{S}$ un subespacio cerrado de $\mathcal{H}$. Supongamos que $A \in L(\mathcal{H})$ es J-autoadjunto y satisface la PFU. Si $A=D D^{\#}$ con $D \in L(\mathcal{K}, \mathcal{H}), N(D)=\{0\}$, supongamos que $\mathcal{M}=\overline{D^{\#}(\mathcal{S})}$ es un subespacio de Krein de $\mathcal{K}$. Luego,

1. si $\mathcal{M}$ es $J$-no negativo entonces $A_{/[\mathcal{S}]}=\inf _{\leq J}\left\{Q^{\#} A Q: Q \in \mathcal{Q}(\mathcal{H}), N(Q)=\mathcal{S}\right\}$.

2. si $\mathcal{M}$ es $J$-no positivo entonces $A_{/[\mathcal{S}]}=\sup _{\leq J}\left\{Q^{\#} A Q: Q \in \mathcal{Q}(\mathcal{H}), N(Q)=\mathcal{S}\right\}$.

Cabe destacar que el orden $\leq_{J}$ considerado en los enunciados anteriores es aquel inducido sobre $L(\mathcal{H})$ por el cono de operadores $J_{\mathcal{H}}$-positivos.

El cono de operadores $J_{\mathcal{H}}$-positivos es el tema central de la Sección 4.2. Estos operadores tienen la propiedad de factorización única y, además, podemos elegir el espacio vectorial $\mathcal{K}$ (que cumple el rol de dominio del factor $D$ de la factorización $A=D D^{\#}$ ) de manera que resulte un espacio de Hilbert.

Notemos que, si $\mathcal{K}$ es un espacio de Hilbert y $\mathcal{S}$ es cualquier subespacio cerrado de $\mathcal{H}$, el subespacio $\mathcal{M}=\overline{D^{\#}(\mathcal{S})}$ es un subespacio cerrado de $\mathcal{K}$ y por lo tanto resulta un "subespacio de Krein" de $\mathcal{K}$. Entonces, el complemento de Schur $A_{/[\mathcal{S}]}$ está bien definido para todo subespacio cerrado $\mathcal{S}$ de $\mathcal{H}$ y además,

$$
A_{/[\mathcal{S}]}=J|A|^{1 / 2} P_{J\left(\mathcal{M}^{\perp}\right)}|A|^{1 / 2},
$$

donde $A=J|A|$ es la descomposición polar de $A$ y $P_{J\left(\mathcal{M}^{\perp}\right)} \in L(\mathcal{K})$ es la proyección ortogonal sobre $J\left(\mathcal{M}^{\perp}\right)$. Es importante destacar que $J\left(\mathcal{M}^{\perp}\right)=\left(|A|^{1 / 2}(\mathcal{S})\right)^{\perp}$. Entonces, de la ecuación anterior, podemos concluir que, si $A \in L(\mathcal{H})$ es $J$-positivo entonces

$$
A_{/[\mathcal{S}]}=J\left(|A|_{/ \mathcal{S}}\right),
$$

donde $|A|_{/ \mathcal{S}}$ es el operador cortocircuito (en el sentido de operadores actuando sobre un espacio de Hilbert) de $|A|$ a $\mathcal{S}$.

En consecuencia, el complemento de Schur de un operador $J$-positivo $A$, actuando en un espacio de Krein $\mathcal{H}$, está intimamente relacionado con el complemento de Schur del operador positivo $J A$, el cual actúa en el espacio de Hilbert $|\mathcal{H}|$. Por lo tanto, muchos resultados clásicos de operadores cortocircuito pueden traducirse al contexto de un espacio de Krein, por ejemplo, si $\mathcal{S}$ y $\mathcal{T}$ son subespacios cerrados de $\mathcal{H}$ y $A, B \in L(\mathcal{H})$ son $J$-positivos,

1. $A_{/[\mathcal{S}]}=\operatorname{máx}_{\leq_{J}}\left\{X \in L(\mathcal{H}): 0 \leq_{J} X \leq_{J} A, R(X) \subseteq \mathcal{S}^{[\perp]}\right\} ;$

2. $A_{/[\mathcal{S}]}=\inf _{\leq J}\left\{Q^{\#} A Q: Q \in \mathcal{Q}(\mathcal{H}), N(Q)=\mathcal{S}\right\}$;

3. si $A \leq_{J} B$ entonces $A_{/[\mathcal{S}]} \leq_{J} B_{/[\mathcal{S}]}$;

4. si $\mathcal{T} \subseteq \mathcal{S}$ entonces $A_{/[\mathcal{S}]} \leq_{J} A_{/[\mathcal{T}]}$.

Los resultados presentados en el Capítulo 4 forman parte del artículo "Schur complements in Krein spaces", publicado en Integral Equations and Operator Theory durante 2007.

En el Capítulo 5 estudiaremos problemas similares a los estudiados por G. Corach y A. Maestripieri en [18], pero considerando métricas indefinidas en lugar de productos internos degenerados. 
Dados un operador de rango cerrado $C \in L(\mathcal{H}, \mathcal{K})$ actuando entre espacios de Hilbert y un vector $y \in \mathcal{K}$, si perturbamos el producto interno de $\mathcal{K}$ con un operador autoadjunto $B \in L(\mathcal{K})$, queda planteado el problema de minimizar (de ser posible) el funcional lineal (indefinido) sobre $\mathcal{H}$ dado por

$$
x \longmapsto\langle C x-y, C x-y\rangle_{B}, \quad x \in \mathcal{H} .
$$

En caso que exista un vector $u \in \mathcal{H}$ tal que

$$
\langle C u-y, C u-y\rangle_{B}=\min _{x \in \mathcal{H}}\langle C x-y, C x-y\rangle_{B},
$$

diremos que $u$ es una $B$-solución de cuadrados mínimos de la ecuación $C x=y$ (lo abreviaremos $B$-LSS, por las iniciales de " $B$-least squares solution").

En primer lugar, utilizando un simple argumento geométrico, probaremos que la existencia de una $B$-LSS $u \in \mathcal{H}$ de la ecuación $C x=y$ es equivalente a que $R(C)$ sea un subespacio $B$-no negativo de $\mathcal{K}$ y que el vector $y-C u$ sea $B$-ortogonal a $R(C)$.

Notemos que $y-C u \perp_{B} R(C)$ si y sólo si $\langle B(C u-y), C x\rangle=0$ para todo $x \in \mathcal{H}$, o equivalentemente, si $u$ es una solución de la ecuación normal:

$$
C^{*} B(C x-y)=0,
$$

por lo que nos enfocaremos en caracterizar y parametrizar las soluciones de (26). En general, si $u \in \mathcal{H}$ es una solución de (26), el conjunto de todas las soluciones de la ecuación normal coincide con la variedad afín

$$
u_{0}+N\left(C^{*} B C\right) .
$$

Además, cabe destacar que la ecuación normal $C^{*} B(C x-y)=0$ admite alguna solución si y sólo si $y \in R(C)+R(C)^{\perp_{B}}$ y, consecuentemente, la ecuación normal admite solución para todo $y \in \mathcal{K}$ si y sólo si el par $(B, R(C))$ es compatible.

En [40], dados un espacio de Hilbert $\mathcal{H}$ de dimensión finita, un operador autoadjunto $B \in$ $L(\mathcal{H})$ y un vector $y \in \mathcal{H}$, B. Hassibi et al. estudiaron el problema de encontrar vectores $u \in \mathcal{S}$ (para un subespacio cerrado $B$-no negativo $\mathcal{S}$ de $\mathcal{H}$ ) tales que

$$
\langle u-y, u-y\rangle_{B}=\min _{s \in \mathcal{S}}\langle s-y, s-y\rangle_{B}=\min _{x \in \mathcal{H}}\left\langle P_{\mathcal{S}} x-y, P_{\mathcal{S}} x-y\right\rangle_{B},
$$

siendo $P_{\mathcal{S}}$ la proyección ortogonal sobre $\mathcal{S}$. Ellos estaban particularmente interesados en aquellos casos en los que hay una única solución del problema. Como $u \in \mathcal{S}$ satisface la Ec. (27) si y sólo si $y-u \in R\left(B P_{\mathcal{S}}\right)^{\perp}$, es fácil ver que esta condición se satisface si y sólo si

$$
P_{\mathcal{S}} B P_{\mathcal{S}} u=P_{\mathcal{S}} B y
$$

Cuando $\mathcal{H}$ es un espacio de Hilbert de dimensión finita, si existe una única solución $u \in \mathcal{S}$ de (28) para algún $y_{0} \in \mathcal{H}$, entonces el operador $\left.P_{\mathcal{S}} B P_{\mathcal{S}}\right|_{\mathcal{S}}$ es inyectivo. Por lo tanto, $\left.P_{\mathcal{S}} B P_{\mathcal{S}}\right|_{\mathcal{S}}$ es inversible, y existe una única solución de $(28)$ para todo $y \in \mathcal{H}$.

En cambio, si $\mathcal{H}$ es un espacio de Hilbert de dimensión infinita, el comentario anterior puede ser falso, ya que $\left.P_{\mathcal{S}} B P_{\mathcal{S}}\right|_{\mathcal{S}}$ puede ser inyectivo sin ser inversible. De hecho, en este caso, existe una solución de la Ec. (28) para todo $y \in \mathcal{H}$ si y sólo si la ecuación

$$
\left(P_{\mathcal{S}} B P_{\mathcal{S}}\right) X=P_{\mathcal{S}} B
$$


admite una solución en $L(\mathcal{H})$, o equivalentemente, el par $(B, \mathcal{S})$ es compatible.

Por lo expuesto anteriormente, la compatibilidad del par $(B, R(C))$ resulta ser una hipótesis razonable para estudiar las $B$-LSS de la ecuación $C x=y$. Suponiendo que el par $(B, R(C))$ es compatible e $y \in \mathcal{K} \backslash R(C)$, es fácil probar que $u \in \mathcal{H}$ es una solución de $C^{*} B(C x-y)=0$ si y sólo si

$$
C u=Q y \text { para alguna } Q \in \mathcal{P}(B, R(C)) .
$$

Luego, si consideramos la solución minimal de la Ec. (26), $u_{y}=C^{\dagger} P_{B, R(C)} y$, podemos parametrizar el conjunto de todas las soluciones de la ecuación normal como

$$
u_{y}+N(B C) .
$$

A manera de resumen de los resultados mencionados anteriormente, enunciaremos el siguiente teorema:

Teorema. Dado $B \in L(\mathcal{K})^{s}$, sea $C \in L(\mathcal{H}, \mathcal{K})$ con rango cerrado tal que $R(C)$ es $B$-no negativo. Luego,

1. dado $y \in \mathcal{K}, u \in \mathcal{H}$ es una $B$-LSS de la ecuación $C x=y$ si y sólo si u es una solución de la ecuación normal $C^{*} B(C x-y)=0$;

2. existe una $B-L S S$ de la ecuación $C x=y$ para todo $y \in \mathcal{K}$ si y sólo si el par $(B, R(C))$ es compatible. En este caso, si $y \in \mathcal{K} \backslash R(C), u \in \mathcal{H}$ es una $B-L S S$ de $C x=y$ si y sólo si existe $Q \in \mathcal{P}(B, R(C))$ tal que $C u=Q y$.

3. si $(B, R(C))$ es compatible, el conjunto de B-LSS de la ecuación $C x=y$ coincide con la variedad afin $u_{y}+N(B C)$.

Recordemos que, dado $y \in \mathcal{K}$, el problema de cuadrados mínimos clásico (en espacios de Hilbert) asociado a la ecuación $C x=y$ admite una (única) solución de norma mínima, más precisamente, aquella definida por $u=C^{\dagger} y$.

El siguiente paso en nuestro estudio es un problema de minimización en el conjunto de $B$ LSS de $C x=y$. Dados $y \in \mathcal{K}, C \in L(\mathcal{H}, \mathcal{K})$ de rango cerrado y un operador autoadjunto $B_{2} \in L(\mathcal{K})$ tales que $\left(B_{2}, R(C)\right)$ es compatible y $R(C)$ es $B_{2}$-no negativo, consideremos el conjunto $u_{y}+N\left(B_{2} C\right)$ de $B_{2}$-LSS de la ecuación $C x=y$.

Ahora, si perturbamos el producto interno de $\mathcal{H}$ con un operador autoadjunto $B \in L(\mathcal{H})$, intentaremos caracterizar las $B_{1} B_{2}$-soluciones de cuadrados mínimos (ó brevemente, $B_{1} B_{2}$-LSS) de $C x=y$, es decir, aquellas $B_{2}$-LSS $w \in \mathcal{H}$ de $C x=y$ tales que

$$
\langle w, w\rangle_{B_{1}} \leq\langle u, u\rangle_{B_{1}}, \quad \text { para todo } u \in u_{y}+N\left(B_{2} C\right) .
$$

De hecho, mostraremos que existe una $B_{1} B_{2}$-LSS de la ecuación $C x=y$ para todo $y \in \mathcal{K}$ si y sólo si $\left(B_{1}, N\left(B_{2} C\right)\right)$ es compatible y $N\left(B_{2} C\right)$ es $B_{1}$-no negativo. Además, si $y \in \mathcal{K} \backslash R\left(B_{2} C\right)^{\perp}$, $w \in \mathcal{H}$ es una $B_{1} B_{2}$-LSS de $C x=y$ si y sólo si existen proyecciones $Q \in \mathcal{P}\left(B_{1}, N\left(B_{2} C\right)\right)$ y $P \in \mathcal{P}\left(B_{2}, R(C)\right)$ tales que

$$
w=(I-Q) C^{\dagger} P y .
$$

Dado $y \in \mathcal{K} \backslash R\left(B_{2} C\right)^{\perp}$, el conjunto de $B_{1} B_{2}$-LSS de la ecuación $C x=y$ puede parametrizarse como

$$
\left\{(I-Q) C^{\dagger} P_{B_{2}, R(C)} y: Q \in \mathcal{P}\left(B_{1}, N\left(B_{2} C\right)\right)\right\} .
$$


Por otra parte, si $y \in R\left(B_{2} C\right)^{\perp}$, el conjunto formado por todas las $B_{1} B_{2}$-LSS de $C x=y$ es $N\left(B_{1}\right) \cap N\left(B_{2} C\right)$ y

$$
\left\{(I-Q) C^{\dagger} P_{B_{2}, R(C)} y: Q \in \mathcal{P}\left(B_{1}, N\left(B_{2} C\right)\right)\right\} \subsetneq N\left(B_{1}\right) \cap N\left(B_{2} C\right) .
$$

Sin embargo, este subconjunto propio contiene a la $B_{1} B_{2}$-LSS de norma mínima.

Proposición. Sean $C \in L(\mathcal{H}, \mathcal{K})$ de rango cerrado y $B_{2} \in L(\mathcal{K})$ autoadjunto tales que para todo $y \in \mathcal{K}$ existen $B_{2}-L S S$ de la ecuación $C x=y$. Dado un operador autoadjunto $B_{1} \in L(\mathcal{H})$, supongamos además que para todo $y \in \mathcal{K}$ existen $B_{1} B_{2}-L S S$ de la ecuación $C x=y$. Entonces,

$$
v_{y}=\left(I-P_{B_{1}, N\left(B_{2} C\right)}\right) C^{\dagger} P_{B_{2}, R(C)} y,
$$

es el único elemento de norma mínima en el conjunto de $B_{1} B_{2}$-LSS de $C x=y$.

Supongamos por un momento que $R(C) \cap N(B)=\{0\}$, y en consecuencia, $N\left(B_{2} C\right)=N(C)$. Por la parametrización dada más arriba, sabemos que $w \in \mathcal{H}$ es una $B_{1} B_{2}$-LSS de la ecuación $C x=y$ si y sólo si

$$
w=(I-Q) C^{\dagger} P_{B_{2}, R(C)} y,
$$

siendo $Q \in \mathcal{P}\left(B_{1}, N(C)\right)$. Esto nos motiva a estudiar el siguiente conjunto de operadores:

$$
G I\left(C, B_{1}, B_{2}\right)=\left\{(I-Q) C^{\dagger} P: Q \in \mathcal{P}\left(B_{1}, N(C)\right) \text { y } P \in \mathcal{P}\left(B_{2}, R(C)\right)\right\} .
$$

Dadas proyecciones $Q \in \mathcal{P}\left(B_{1}, N(C)\right)$ y $P \in \mathcal{P}\left(B_{2}, R(C)\right)$, consideremos $D=(I-Q) C^{\dagger} P$. Utilizando la propiedades de $C^{\dagger}$, es fácil ver que $D$ es una inversa generalizada pesada de $C$, ya que $D$ es una solución del sistema de ecuaciones

$$
C X C=C, \quad X C X=X, \quad B_{1}(X C)=(X C)^{*} B_{1}, \quad B_{2}(C X)=(C X)^{*} B_{2} .
$$

La Sección 5.2 está dedicada a presentar condiciones necesarias y suficientes para la existencia de inversas generalizadas pesadas de un operador $C$ con rango cerrado, y a caracterizar estas inversas en términos de la inversa de Moore-Penrose de $C$ y los conjuntos de proyecciones $B_{i^{-}}$ autoadjuntas (para $i=1,2)$. De hecho, probaremos que, si $\left(B_{1}, N(C)\right)$ y $\left(B_{2}, R(C)\right)$ son pares compatibles, el conjunto $G I\left(C, B_{1}, B_{2}\right)$ coincide con el conjunto de soluciones de (30).

Finalmente, recordemos que, si $R(C)$ es un subespacio $B$-indefinido, la ecuación $C x=y$ no admite $B$-soluciones de cuadrados mínimos aunque existan soluciones de la ecuación normal. La Sección 5.3 está dedicada a relacionar a las soluciones de $C^{*} B(C x-y)=0$ con las soluciones de un problema de minimax. 


\section{Capítulo 1}

\section{Preliminares}

Comenzaremos fijando algunas notaciones que utilizaremos a continuación. Dado un espacio de Hilbert $\mathcal{H}$ con producto interno $\langle$,$\rangle , denotaremos con L(\mathcal{H})$ al álgebra de operadores lineales acotados sobre $\mathcal{H}$. $L(\mathcal{H})^{s}$ es el subespacio (real) de $L(\mathcal{H})$ formado por los operadores autoadjuntos, $L(\mathcal{H})^{+}$es el cono de operadores (semidefinidos) positivos de $L(\mathcal{H}), G L(\mathcal{H})$ es el grupo de operadores inversibles de $L(\mathcal{H}), G L(\mathcal{H})^{s}=G L(\mathcal{H}) \cap L(\mathcal{H})^{s}$ y $G L(\mathcal{H})^{+}=G L(\mathcal{H}) \cap L(\mathcal{H})^{+}$. Fijado un operador $T \in L(\mathcal{H})$, notaremos $R(T)$ al rango de $T$ y $N(T)$ a su núcleo.

Con $\mathcal{Q}=\mathcal{Q}(L(\mathcal{H}))$ denotaremos al conjunto de proyecciones en $L(\mathcal{H})$ y con $\mathcal{P}=\mathcal{P}(L(\mathcal{H}))$ al subconjunto de $\mathcal{Q}$ de proyecciones ortogonales, i.e.

$$
\mathcal{Q}=\left\{Q \in L(\mathcal{H}): Q^{2}=Q\right\} \quad \text { y } \quad \mathcal{P}=\left\{P \in L(\mathcal{H}): P^{2}=P=P^{*}\right\} .
$$

Si $Q \in \mathcal{Q} \backslash \mathcal{P}$, diremos que $Q$ es una proyección oblicua.

Dados dos subespacios $\mathcal{S}$ y $\mathcal{T}$ de $\mathcal{H}$, denotaremos $\mathcal{S}+\mathcal{T}$ a la suma directa (interna) de $\mathcal{S}$ y $\mathcal{T}$, $\mathcal{S} \oplus \mathcal{T}$ a la suma directa ortogonal de ellos, y $\mathcal{S} \ominus \mathcal{T}=\mathcal{S} \cap(\mathcal{S} \cap \mathcal{T})^{\perp}$. Si $\mathcal{H}=\mathcal{S}+\mathcal{T}$, la proyección sobre $\mathcal{S}$ a lo largo de $\mathcal{T}, P_{\mathcal{S} / / \mathcal{T}}$, es la (única) proyección con $R\left(P_{\mathcal{S} / / \mathcal{T}}\right)=\mathcal{S}$ y $N\left(P_{\mathcal{S} / / \mathcal{T}}\right)=\mathcal{T}$. En particular, $P_{\mathcal{S}}=P_{\mathcal{S} / \mathcal{S}^{\perp}}$ es la proyección ortogonal sobre $\mathcal{S}$.

\subsection{Teoría de operadores actuando sobre un espacio de Hilbert}

\subsubsection{Factorización de Douglas y descomposición polar}

El siguiente resultado, debido a R. G. Douglas [28], caracteriza inclusiones de rangos de operadores.

Teorema 1.1.1. Dados espacios de Hilbert $\mathcal{H}, \mathcal{K}_{1}, \mathcal{K}_{2}$ y operadores $A \in L\left(\mathcal{K}_{1}, \mathcal{H}\right)$ y $B \in$ $L\left(\mathcal{K}_{2}, \mathcal{H}\right)$, las siguientes condiciones son equivalentes:

1. la ecuación $A X=B$ tiene una solución en $L\left(\mathcal{K}_{2}, \mathcal{K}_{1}\right)$;

2. $R(B) \subseteq R(A)$;

3. existe $\lambda>0$ tal que $B B^{*} \leq \lambda A A^{*}$.

En este caso, existe un único $D \in L\left(\mathcal{K}_{2}, \mathcal{K}_{1}\right)$ tal que $A D=B$ y $R(D) \subseteq \overline{R\left(A^{*}\right)}$; además cumple que $N(D)=N(B)$ y $\|D\|=\inf \left\{\lambda>0: B B^{*} \leq \lambda A A^{*}\right\}$. El operador $D$ se denomina solución reducida de la ecuación $A X=B$. 
Demostración. En primer lugar, notemos que la implicación 1. $\Rightarrow$ 2. es trivial.

1. $\Rightarrow$ 3. : Si $B=A C$ con $C \in L\left(\mathcal{K}_{2}, \mathcal{K}_{1}\right)$ entonces $B B^{*}=A C C^{*} A^{*} \leq\|C\|^{2} A A^{*}$, pues $C C^{*} \leq$ $\|C\|^{2} I$, es decir, vale 2 .

2. $\Rightarrow$ 1. : Sean $A \in L\left(\mathcal{K}_{1}, \mathcal{H}\right)$ y $B \in L\left(\mathcal{K}_{2}, \mathcal{H}\right)$ tales que $R(B) \subseteq R(A)$. Podemos definir un operador $C: \mathcal{K}_{2} \rightarrow \mathcal{K}_{1}$ de la siguiente manera: si $x \in \mathcal{K}_{2}$, tenemos que $B x \in R(B) \subseteq R(A)$ y, como $A: N(A)^{\perp} \rightarrow R(A)$ es biyectivo, existe un único $y \in N(A)^{\perp}$ tal que $A y=B x$. Definiendo $C x=y$ tenemos que $C$ está bien definido y $B=A C$.

Falta probar que $C$ es acotado. Como $C$ está definido en todo $\mathcal{K}_{2}$, es suficiente mostrar que $C$ tiene gráfico cerrado. Consideremos una sucesión $\left\{\left(x_{n}, y_{n}\right)\right\}_{n \in \mathbb{N}}$, con $y_{n}=C_{1} x_{n}$, tal que $\left(x_{n}, y_{n}\right) \rightarrow(x, y)$. Por definición, $B x_{n}=A y_{n}$ para todo $n \in \mathbb{N}$. Como $A$ y $B$ son acotados, $\lim _{n \rightarrow \infty} A y_{n}=A y$ y $\lim _{n \rightarrow \infty} B x_{n}=B x$, con lo cual $A y=B x$. Además, como $N(A)^{\perp}$ es cerrado, tenemos que $y \in N(A)^{\perp}$ y por consiguiente $C x=y$. Luego, $C$ es acotado.

3. $\Rightarrow$ 1. : Supongamos que $B B^{*} \leq \lambda A A^{*}$ para algún $\lambda \geq 0$ y definamos la siguiente aplicación $D: R\left(A^{*}\right) \rightarrow R\left(B^{*}\right):$

$$
D\left(A^{*} x\right)=B^{*} x, \quad \text { si } x \in \mathcal{H} .
$$

Entonces $D$ está bien definida pues, si $A^{*} x=A^{*} x^{\prime}$ entonces $A^{*}\left(x-x^{\prime}\right)=0 \mathrm{y}$, como $B B^{*} \leq \lambda A A^{*}$, también $x-x^{\prime} \in N\left(B^{*}\right)$ o equivalentemente $B^{*} x=B^{*} x^{\prime}$. Veamos que $D$ es acotada en $R\left(A^{*}\right)$ :

$$
\left\|D\left(A^{*} x\right)\right\|^{2}=\left\|B^{*} f\right\|^{2}=\left\langle B B^{*} x, x\right\rangle \leq \lambda^{2}\left\langle A A^{*} x, x\right\rangle=\lambda^{2}\left\|A^{*} x\right\|^{2} .
$$

Por lo tanto, $D$ puede ser extendido de manera única a $\overline{R\left(A^{*}\right)}$ y, si definimos $D x=0$ para $x \in R\left(A^{*}\right)^{\perp}$, entonces $D \in L\left(\mathcal{K}_{1}, \mathcal{K}_{2}\right)$ y $D B^{*}=A^{*}$, o equivalentemente, $A=B D^{*}$ con $D^{*} \in$ $L\left(\mathcal{K}_{2}, \mathcal{K}_{1}\right)$.

Finalmente, probemos que existe una única solución reducida de la ecuación $A X=B$. Notemos que la inclusión $R(D) \subseteq \overline{R\left(A^{*}\right)}=N(A)^{\perp}$ identifica unívocamente al operador $D$, ya que $A D=B$ y $A_{\mid N(A)^{\perp}}$ es inyectivo. En particular, los operadores construidos en $2 . \Rightarrow 1$. y 3. $\Rightarrow 1$. tienen el rango contenido en el subespacio $\overline{R\left(A^{*}\right)}=N(A)^{\perp}$; por lo tanto, estos operadores coinciden.

Corolario 1.1.2. Si $A \in L(\mathcal{H}, \mathcal{K})$ entonces $R\left(\left(A A^{*}\right)^{1 / 2}\right)=R(A)$.

Demostración. Dado que $A A^{*}=\left(A A^{*}\right)^{1 / 2}\left(\left(A A^{*}\right)^{1 / 2}\right)^{*}$, la igualdad entre los rangos de $A$ y $\left(A A^{*}\right)^{1 / 2}$ es consecuencia del Teorema de Douglas.

Corolario 1.1.3. Sean $A \in L(\mathcal{H}), Q \in \mathcal{Q}$ y supongamos que $R(Q A) \subseteq R(A)$. Entonces, la solución reducida $D \in L(\mathcal{H})$ de la ecuación $A X=Q A$ es una proyección, es decir, $D \in \mathcal{Q}$.

Demostración. En primer lugar, notemos que, si $D$ es la solución reducida de $A X=Q A$ entonces $A D^{2}=Q A D=Q^{2} A=Q A$ y $R\left(D^{2}\right) \subseteq R(D) \subseteq \overline{R\left(A^{*}\right)}$. Luego, $D^{2}$ también es una solución reducida de la ecuación $A X=Q A$ y, por la unicidad de la misma, resulta que $D \in \mathcal{Q}$.

Definición. Fijado un espacio de Hilbert $\mathcal{H}$, una isometría parcial es un operador $W \in L(\mathcal{H})$ tal que $\|W x\|=\|x\|$ para todo $x \in N(W)^{\perp}$. Llamaremos espacio inicial de $W$ al subespacio $N(W)^{\perp}$, y espacio final de $W$ al subespacio $R(W)$. 
Notemos que, por la identidad de polarización, si $W \in L(\mathcal{H})$ es una isometría parcial y $x, y \in$ $N(W)^{\perp}$,

$$
\langle W x, W y\rangle=\sum_{k=0}^{3} i^{k}\left\|W x+i^{k} W y\right\|^{2}=\sum_{k=0}^{3} i^{k}\left\|W\left(x+i^{k} y\right)\right\|^{2}=\sum_{k=0}^{3} i^{k}\left\|x+i^{k} y\right\|^{2}=\langle x, y\rangle .
$$

Proposición 1.1.4. Si $W \in L(\mathcal{H})$, son equivalentes:

1. W es una isometría parcial;

2. $E=W^{*} W$ es la proyección ortogonal sobre $N(W)^{\perp}$;

3. $W^{*} W W^{*}=W^{*}$;

Además, en este caso, $R(W)$ es un subespacio cerrado. Luego, si $W$ es una isometría parcial con espacio inicial $N(W)^{\perp}$ y espacio final $R(W)$, entonces $W^{*}$ es una isometría parcial con espacio inicial $R(W)$ y espacio final $N(W)^{\perp}$.

Demostración. 1. $\Leftrightarrow$ 2.: Supongamos que $W \in L(\mathcal{H})$ es una isometría parcial. Luego, si $x \in$ $N(W)^{\perp}$, aplicando la Ec. (1.1.1) vemos que

$\left\langle W^{*} W x, y\right\rangle=\langle W x, W y\rangle=\left\langle W x, W P_{N(W)^{\perp}} y\right\rangle=\left\langle x, P_{N(W)^{\perp}} y\right\rangle=\langle x, y\rangle$ para todo $y \in \mathcal{H}$.

Por lo tanto, $W^{*} W x=x$ para todo $x \in N(W)^{\perp}$ y $W^{*} W z=0$ para todo $z \in N(W)$. Por lo tanto, $E=W^{*} W$ es la proyección ortogonal sobre $N(W)^{\perp}$. Recíprocamente, si $E=W^{*} W$ es la proyección ortogonal sobre $N(W)^{\perp}$,

$$
\|W x\|^{2}=\langle W x, W x\rangle=\left\langle W^{*} W x, x\right\rangle=\langle x, x\rangle \quad \text { para todo } x \in N(W)^{\perp},
$$

i.e. $W$ es una isometría parcial.

2. $\Leftrightarrow$ 3.: Si $E=W^{*} W$ es la proyección ortogonal sobre $N(W)^{\perp}$, entonces $W^{*} W W^{*}=W^{*}$ ya que $R\left(W^{*}\right) \subseteq N(W)^{\perp}$.

Recíprocamente, si $W^{*} W W^{*}=W^{*}$, tenemos que $\left(W^{*} W\right)^{2}=\left(W^{*} W W^{*}\right) W=W^{*} W$ y además $\left(W^{*} W\right)^{*}=W^{*} W$. Por lo tanto, $E=W^{*} W$ es una proyección ortogonal. Como $E W^{*}=$ $W^{*}$, tenemos que $R\left(W^{*}\right) \subseteq R(E)$. Más aún, $N(W)^{\perp}=\overline{R\left(W^{*}\right)} \subseteq R(E)$ porque $R(E)$ es cerrado. También es fácil ver que $N(W) \subseteq N\left(W^{*} W\right)=N(E)$, con lo que resulta que $E$ es la proyección ortogonal sobre $N(W)^{\perp}$.

Notemos además que, si $W^{*} W W^{*}=W^{*}$, entonces $R\left(W^{*}\right)=R\left(W^{*} W W^{*}\right) \subseteq R\left(W^{*} W\right) \subseteq$ $R\left(W^{*}\right)$. Es decir, $R\left(W^{*}\right)=R\left(W^{*} W\right)$ es cerrado ya que $E=W^{*} W$ es una proyección. Por lo tanto, $R(W)$ también es cerrado.

Dado un operador $A \in L(\mathcal{H})$, el módulo de $A$ se define como $|A|=\left(A^{*} A\right)^{1 / 2}$. Es decir, el operador $|A|$ es la (única) raíz cuadrada positiva del operador $A^{*} A$.

Teorema 1.1.5 (Descomposición polar). Si $A \in L(\mathcal{H})$ existe una isometría parcial $W$ con espacio inicial $N(A)^{\perp}$ y espacio final $\overline{R(A)}$ tal que $A=W|A|$. Además, si $A=U P$ con $P \in$ $L(\mathcal{H})^{+}$y $U$ una isometría parcial con espacios inicial y final $N(A)^{\perp}$ y $\overline{R(A)}$, respectivamente, entonces $P=|A|$ y $U=W$. 
Demostración. Si $x \in \mathcal{H}$, entonces $\|A x\|^{2}=\langle A x, A x\rangle=\left\langle A^{*} A x, x\right\rangle=\langle|A| x,|A| x\rangle$. Luego,

$$
\|A x\|^{2}=\||A| x\|^{2} \quad \text { para todo } x \in \mathcal{H} .
$$

Como $R\left(A^{*}\right)^{\perp}=N(A)$, vemos que $R\left(A^{*}\right)$ es un subespacio denso en $N(A)^{\perp}$. Además, si $y \in$ $R\left(A^{*}\right)$, existe $x \in N\left(A^{*}\right)^{\perp}=\overline{R(A)}$ tal que $y=A^{*} x$. Por lo tanto, el conjunto $\left\{A^{*} A x: x \in \mathcal{H}\right\}$ es denso en $\overline{R\left(A^{*}\right)}=N(A)^{\perp}$.

Si definimos $W: R(|A|) \rightarrow R(A)$ mediante $W(|A| x)=A x$, la Ec. (1.1.2) asegura que $W$ está bien definido y es una isometría. Luego, $W$ puede extenderse a una isometría $W$ : $N(A)^{\perp} \rightarrow \overline{R(A)}$ y, definiendo $W x=0$ para $x \in N(A)$, tenemos que $W$ es una isometría parcial. Por definición $W$ cumple $W|A|=A$

Para probar la unicidad, notemos que, si $A=U P$ entonces $A^{*} A=P U^{*} U P$. Pero $E=U^{*} U$ es la proyección ortogonal sobre el espacio inicial de $U, N(A)^{\perp}$, y además $N(A)^{\perp} \subseteq N(P)^{\perp}=\overline{R(P)}$ ya que $N(P) \subseteq N(A)$ y $P \in L(\mathcal{H})^{+}$. Luego, $E P=P$ y $A^{*} A=P E P=P^{2}$. Por la unicidad de la raíz cuadrada positiva, podemos afirmar que $P=|A|$. Finalmente, como $A=U|A|$, tenemos que $U|A| x=A x=W|A| x$ para todo $x \in \mathcal{H}$. Es decir, $U$ y $W$ coinciden en un subconjunto denso de su espacio inicial en común. Por lo tanto, $U=W$.

Corolario 1.1.6. Si $A \in L(\mathcal{H})$ tiene descomposición polar $A=W|A|$ entonces la descomposición polar de $A^{*}$ es $W^{*}\left|A^{*}\right|$.

Demostración. Recordemos que, si $W$ es una isometría parcial de $N(A)^{\perp}$ en $\overline{R(A)}$, entonces $E=W^{*} W$ es la proyección ortogonal sobre $N(A)^{\perp}$ y $F=W W^{*}$ es la proyección ortogonal sobre $\overline{R(A)}$. Además, $N(|A|)=N(A)$ implica que $E|A|=|A|$, y luego $A^{*}=|A| W^{*}=E|A| W^{*}=$ $W^{*}\left(W|A| W^{*}\right)$. Finalmente, notemos que $W|A| W^{*} \in L(\mathcal{H})^{+}$y, por la Proposición 1.1.4, $W^{*}$ es una isometría parcial entre $N\left(A^{*}\right)^{\perp}=\overline{R(A)}$ y $\overline{R\left(A^{*}\right)}=N(A)^{\perp}$. Entonces, por la unicidad de la descomposición polar, tenemos que $\left|A^{*}\right|=W|A| W^{*}$ y que la isometría parcial de la descomposición polar de $A^{*}$ es $W^{*}$.

Observación 1.1.7. Si $B \in L(\mathcal{H})$ es un operador autoadjunto y su descomposición polar es $B=W|B|$, siendo $W$ una isometría parcial, entonces

- $W=W^{*}$

- $W$ y $|B|$ conmutan;

- Los espacios inicial y final de $W$ coinciden con $N(B)^{\perp}$;

- $N(|B|)=N(B)$.

De hecho, si $B=B^{*}$ luego $W|B|=B=B^{*}=W^{*}\left|B^{*}\right|=W^{*}|B|$, y por la unicidad de la descomposición polar, resulta que $W^{*}=W$. Además, con un argumento similar al anterior, vemos que $W|B|=B=B^{*}=(W|B|)^{*}=|B| W^{*}=|B| W$. Como $B$ es autoadjunto $\overline{R(B)}=$ $N\left(B^{*}\right)^{\perp}=N(B)^{\perp}$, es decir, los espacios inicial y final de $W$ coinciden con $N(B)^{\perp}$. La última afirmación es consecuencia de que $N(W)=N(B)^{\perp}$.

Corolario 1.1.8 (Descomposición polar para operadores autoadjuntos). Si $B \in L(\mathcal{H})^{s}$ existe una reflexión $J \in L(\mathcal{H})$ tal que $A=J|A|$ y $J x=x$ para todo $x \in N(A)$. Además, si $A=U P$ con $P \in L(\mathcal{H})^{+}$y $U$ reflexión tal que $U x=x$ para todo $x \in N(A)$, respectivamente, entonces $P=|A|$ y $U=J$. 
Demostración. Es consecuencia del Teorema 1.1.5 y la observación anterior.

Para finalizar, dado $B \in L(\mathcal{H})^{s}$ con descomposición polar $B=J A$ - siendo $A \in L(\mathcal{H})^{+}$

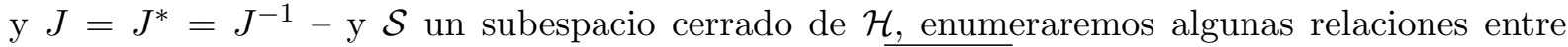
subespacios que utilizaremos con frecuencia. Si $\mathcal{M}=\overline{A^{1 / 2}(\mathcal{S})}$ entonces

- $\mathcal{M}^{\perp}=A^{1 / 2}(\mathcal{S})^{\perp}=A^{-1 / 2}\left(\mathcal{S}^{\perp}\right)$;

- $J(\mathcal{M})^{\perp}=J\left(\mathcal{M}^{\perp}\right)$;

- $A^{-1 / 2}\left(J(\mathcal{M})^{\perp}\right)=B^{-1}\left(\mathcal{S}^{\perp}\right)=B(\mathcal{S})^{\perp}$;

- $N\left(A^{1 / 2}\right) \subseteq \mathcal{M}^{\perp}$ y $N\left(A^{1 / 2}\right) \subseteq J(\mathcal{M})^{\perp}$.

\subsubsection{Operadores densamente definidos}

Definición. Dado un espacio de Hilbert $\mathcal{H}$, un operador es una aplicación lineal $T$ tal que su dominio Dom $T$ y su rango $R(T)$ son subespacios de $\mathcal{H}$.

En estos párrafos no supondremos que $T$ es continuo (o acotado), sino todo lo contrario. De hecho, si $T$ es continuo (relativo a la topología de la norma que Dom $T$ hereda de $\mathcal{H}$ ) entonces $T$ tiene una extensión continua a la clausura de $\operatorname{Dom} T$, y por consiguiente a $\mathcal{H}$, ya que $\overline{\operatorname{Dom} T}$ es un subespacio complementado en $\mathcal{H}$.

El gráfico $\operatorname{Gr}(T)$ de un operador $T$ es el subespacio de $\mathcal{H} \times \mathcal{H}$ formado por los pares $(x, T x)$, donde $x \in \operatorname{Dom} T$. Es fácil ver que $S$ es una extensión de $T$ (es decir, $\operatorname{Dom} T \subset \operatorname{Dom} T$ y $S x=T x$ para todo $x \in \operatorname{Dom} T)$ si y sólo si

$$
G r(T) \subset G r(S) .
$$

Generalmente notaremos esta inclusión simplemente $S \subset T$.

Un operador $T$ en $\mathcal{H}$ se dice cerrado si su gráfico $G r(T)$ es un subespacio cerrado de $\mathcal{H} \times \mathcal{H}$. Luego, por el Teorema del Gráfico Cerrado, $T \in L(\mathcal{H})$ si y sólo si $\operatorname{Dom} T=\mathcal{H}$ y $T$ es cerrado.

A continuación intentaremos asociar un adjunto $T^{*}$ a un operador dado $T$. Su dominio Dom $T^{*}$ estará formado por aquellos $y \in \mathcal{H}$ para los cuales el funcional lineal

$$
x \longmapsto\langle T x, y\rangle,
$$

es continuo sobre Dom $T$. Si $y \in \operatorname{Dom} T^{*}$, el Teorema de Hahn-Banach asegura que existe un funcional lineal sobre $\mathcal{H}$ que extiende al de la Ec. (1.1.3). Luego, por el Teorema de Representación de Riesz, existe un vector $T^{*} y \in \mathcal{H}$ tal que

$$
\langle T x, y\rangle=\left\langle x, T^{*} y\right\rangle \quad \text { para todo } x \in \operatorname{Dom} T .
$$

Notemos que $T^{*} y$ está unívocamente determinado por la Ec. (1.1.4) si y sólo si Dom $T$ es denso en $\mathcal{H}$, es decir, si $T$ está densamente definido. Por lo tanto, los únicos operadores que admiten un adjunto $T^{*}$ son aquellos que están densamente definidos. Unos pocos cálculos de rutina muestran que $T^{*}$ también es un operador en $\mathcal{H}$.

Las operaciones algebraicas entre operadores no acotados deben tratarse con cuidado, pues hay que considerar los dominios en los cuales definirlas. Las elecciones naturales para el dominio de la suma y el producto de dos operadores $S$ y $T$ son:

$$
\operatorname{Dom}(S+T)=\operatorname{Dom} S \cap \operatorname{Dom} T, \quad \operatorname{Dom}(S T)=\{x \in \operatorname{Dom} T: T x \in \operatorname{Dom} S\} .
$$


Teorema 1.1.9. Supongamos que $S, T$ y $S T$ son operadores densamente definidos en $\mathcal{H}$. Luego,

$$
T^{*} S^{*} \subset(S T)^{*} .
$$

Además, si $S \in L(\mathcal{H})$, entonces $T^{*} S^{*}=(S T)^{*}$.

Definición. Un operador $T$ en $\mathcal{H}$ es simétrico si, para todo $x, y \in \operatorname{Dom} T$,

$$
\langle T x, y\rangle=\langle x, T y\rangle .
$$

Notemos que los operadores densamente definidos simétricos son exactamente aquellos que satisfacen $T \subset T^{*}$.

Por otra parte, si $T=T^{*}$, diremos que $T$ es autoadjunto. Estas dos propiedades evidentemente coinciden cuando $T \in L(\mathcal{H})$, pero en general son condiciones distintas.

Teorema 1.1.10. Si $T$ es un operador densamente definido en $\mathcal{H}$, entonces $T^{*}$ es un operador cerrado. En particular, los operadores autoadjuntos son cerrados.

Teorema 1.1.11. Supongamos que $T$ es un operador densamente definido en $\mathcal{H}$. Luego,

1. Si $\operatorname{Dom} T=\mathcal{H}$ entonces $T$ es autoadjunto y $T \in L(\mathcal{H})$.

2. Si $T$ es cerrado entonces Dom $T^{*}$ es denso en $\mathcal{H}$ y $T^{* *}=T$.

\subsection{3. Ángulos entre subespacios cerrados de un espacio de Hilbert}

La noción de "ángulo" entre un par de subespacios cerrados de un espacio de Hilbert es realmente útil, ya que muchas veces permite dar una interpretación geométrica a resultados que parecen ser puramente analíticos o topológicos. Las siguientes son dos definiciones diferentes de ángulo entre subespacios de un espacio de Hilbert $\mathcal{H}$.

Definición (K. Friedrichs [34]). Dados dos subespacios cerrados $\mathcal{S}$ y $\mathcal{T}$ de $\mathcal{H}$, el ángulo entre ellos es aquel $\alpha(\mathcal{S}, \mathcal{T}) \in\left[0, \frac{\pi}{2}\right]$ cuyo coseno está definido por

$$
c(\mathcal{S}, \mathcal{T})=\sup \{|\langle x, y\rangle|: x \in \mathcal{S} \ominus \mathcal{T},\|x\| \leq 1, y \in \mathcal{T} \ominus \mathcal{S},\|y\| \leq 1\} .
$$

Definición (J. Dixmier [27]). El ángulo mínimo entre $\mathcal{S}$ y $\mathcal{T}$ es el ángulo $\alpha_{0}(\mathcal{S}, \mathcal{T}) \in\left[0, \frac{\pi}{2}\right]$ cuyo coseno está definido por

$$
c_{0}(\mathcal{S}, \mathcal{T})=\sup \{|\langle x, y\rangle|: x \in \mathcal{S},\|x\| \leq 1, y \in \mathcal{T},\|y\| \leq 1\} .
$$

En realidad, Dixmier [27] le otorga a Friedrichs el crédito por la definición de ángulo mínimo. En general $c(\mathcal{S}, \mathcal{T}) \leq c_{0}(\mathcal{S}, \mathcal{T})$, sin embargo, cuando $\mathcal{S} \cap \mathcal{T}=\{0\}$ las definiciones coinciden.

Para poder probar los resultados sobre ángulos que utilizaremos es necesario recordar algunas propiedades básicas sobre proyecciones ortogonales. Los siguientes lemas son bien conocidos y pueden encontrarse en la literatura clásica de análisis funcional, e. g. [17, 37], ó en la recopilación de Deutsch [26].

Lema 1.1.12. Dados dos subespacios cerrados $\mathcal{S}$ y $\mathcal{T}$ de un espacio de Hilbert $\mathcal{H}$,

$$
P_{\mathcal{S}} P_{\mathcal{T}}=P_{\mathcal{T}} P_{\mathcal{S}} \quad \Leftrightarrow \quad P_{\mathcal{S}} P_{\mathcal{T}}=P_{\mathcal{S} \cap \mathcal{T}} \quad \Leftrightarrow \quad P_{\mathcal{S}} P_{\mathcal{T}} \text { es una proyección ortogonal. }
$$


Lema 1.1.13. Dados dos subespacios cerrados $\mathcal{S}$ y $\mathcal{T}$ de un espacio de Hilbert $\mathcal{H}$,

$$
P_{\mathcal{S}} P_{\mathcal{T}}=0 \quad \Leftrightarrow \quad P_{\mathcal{T}} P_{\mathcal{S}}=0 \quad \Leftrightarrow \quad \mathcal{S} \perp \mathcal{T} \text {. }
$$

Lema 1.1.14. Dados dos subespacios cerrados $\mathcal{S}$ y $\mathcal{T}$ de un espacio de Hilbert $\mathcal{H}$,

$$
P_{\mathcal{S}} P_{\mathcal{T}}=P_{\mathcal{S}} \quad \Leftrightarrow \quad P_{\mathcal{T}} P_{\mathcal{S}}=P_{\mathcal{S}} \quad \Leftrightarrow \quad \mathcal{S} \subseteq \mathcal{T}
$$

Lema 1.1.15. Sean $\mathcal{S}$ y $\mathcal{T}$ dos subespacios cerrados de un espacio de Hilbert $\mathcal{H}$. Si $P_{\mathcal{S}}$ y $P_{\mathcal{T}}$ conmutan, entonces

$$
P_{\overline{\mathcal{S}+\mathcal{T}}}=P_{\mathcal{S}}+P_{\mathcal{T}}-P_{\mathcal{S}} P_{\mathcal{T}}
$$

Por lo tanto, si $\mathcal{S} \subseteq \mathcal{T}^{\perp}$ entonces $\mathcal{S}+\mathcal{T}$ es cerrado y $P_{\mathcal{S}+\mathcal{T}}=P_{\mathcal{S}}+P_{\mathcal{T}}$.

Demostración. Sea $Q=P_{\mathcal{S}}+P_{\mathcal{T}}-P_{\mathcal{S}} P_{\mathcal{T}}$. Notemos que, como $P_{\mathcal{S}}$ y $P_{\mathcal{T}}$ conmutan, es fácil ver que $Q \in \mathcal{P}$. Si $x=s+t$, con $s \in \mathcal{S}$ y $t \in \mathcal{T}$, entonces

$$
\begin{aligned}
Q x & =P_{\mathcal{S}}(s+t)+\left(I-P_{\mathcal{S}}\right) P_{\mathcal{T}}(s+t)=s+P_{\mathcal{S}} t+\left(I-P_{\mathcal{S}}\right)\left(P_{\mathcal{T}} s+t\right)= \\
& =s+P_{\mathcal{S}} t+P_{\mathcal{T}}\left(I-P_{\mathcal{S}}\right) s+\left(I-P_{\mathcal{S}}\right) t=s+P_{\mathcal{S}} t+\left(I-P_{\mathcal{S}}\right) t=s+t=x,
\end{aligned}
$$

es decir, $\mathcal{S}+\mathcal{T} \subseteq R(Q)$. Además, como $R(Q)$ es cerrado, tenemos que $\overline{\mathcal{S}+\mathcal{T}} \subseteq R(Q)$. Por otra parte, si $y \in(\overline{\mathcal{S}+\mathcal{T}})^{\perp}=\mathcal{S}^{\perp} \cap \mathcal{T}^{\perp}$, entonces $Q x=0$. Luego, $(\overline{\mathcal{S}+\mathcal{T}})^{\perp} \subseteq N(Q)$ y, por lo tanto, $Q=P_{\overline{\mathcal{S}+\mathcal{T}}}$.

Ahora, supongamos que $\mathcal{S} \subseteq \mathcal{T}^{\perp}$. Entonces $P_{\mathcal{T}} P_{\mathcal{S}}=P_{\mathcal{S}} P_{\mathcal{T}}=0$, con lo cual $P_{\mathcal{S}}$ y $P_{\mathcal{T}}$ conmutan y (1.1.8) implica que $P_{\overline{\mathcal{S}+\mathcal{T}}}=P_{\mathcal{S}}+P_{\mathcal{T}}$. Falta probar que $\mathcal{S}+\mathcal{T}$ es cerrado, pero si $x \in \overline{\mathcal{S}+\mathcal{T}}$, entonces

$$
x=P_{\overline{\mathcal{S}+\mathcal{T}}} x=P_{\mathcal{S}} x+P_{\mathcal{T}} x \in \mathcal{S}+\mathcal{T} .
$$

Lema 1.1.16. Dados dos subespacios cerrados $\mathcal{S}$ y $\mathcal{T}$ de un espacio de Hilbert $\mathcal{H}$,

$$
\begin{array}{rlll}
P_{\mathcal{S}} P_{\mathcal{T}}=P_{\mathcal{T}} P_{\mathcal{S}} & \Leftrightarrow & P_{\mathcal{S}^{\perp}} P_{\mathcal{T}}=P_{\mathcal{T}} P_{\mathcal{S}^{\perp}} \quad \Leftrightarrow \quad P_{\mathcal{S}^{\perp}} P_{\mathcal{T}^{\perp}}=P_{\mathcal{T}^{\perp}} P_{\mathcal{S}^{\perp}} \\
& \Leftrightarrow \quad \mathcal{S}=\mathcal{S} \cap \mathcal{T}+\mathcal{S} \cap \mathcal{T}^{\perp} .
\end{array}
$$

Demostración. La equivalencia de las tres primeras afirmaciones es inmediata, teniendo en cuenta que $P_{\mathcal{S}^{\perp}}=I-P_{\mathcal{S}}$ y $P_{\mathcal{T} \perp}=I-P_{\mathcal{T}}$.

Notemos que, si $P_{\mathcal{S}}$ y $P_{\mathcal{T}}$ conmutan también lo hacen $P_{\mathcal{S}}$ y $P_{\mathcal{T} \perp}$. Luego, por Lema 1.1.12,

$$
P_{\mathcal{S} \cap \mathcal{T}}+P_{\mathcal{S} \cap \mathcal{T}^{\perp}}=P_{\mathcal{S}} P_{\mathcal{T}}+P_{\mathcal{S}} P_{\mathcal{T}^{\perp}}=P_{\mathcal{S}}\left(P_{\mathcal{T}}+P_{\mathcal{T}^{\perp}}\right)=P_{\mathcal{S}}
$$

Entonces $\mathcal{S} \subseteq \mathcal{S} \cap \mathcal{T}+\mathcal{S} \cap \mathcal{T}^{\perp}$. Como la otra inclusión es evidente, tenemos que $\mathcal{S}=\mathcal{S} \cap \mathcal{T}+\mathcal{S} \cap \mathcal{T}^{\perp}$.

Recíprocamente, si $\mathcal{S}=\mathcal{S} \cap \mathcal{T}+\mathcal{S} \cap \mathcal{T}^{\perp}$, entonces por el Lema 1.1.15,

$$
P_{\mathcal{S}}=P_{\mathcal{S} \cap \mathcal{T}+\mathcal{S} \cap \mathcal{T} \perp}=P_{\mathcal{S} \cap \mathcal{T}}+P_{\mathcal{S} \cap \mathcal{T} \perp}
$$

Luego, $P_{\mathcal{T}} P_{\mathcal{S}}=P_{\mathcal{T}} P_{\mathcal{S} \cap \mathcal{T}}+P_{\mathcal{T}} P_{\mathcal{S} \cap \mathcal{T} \perp}=P_{\mathcal{S} \cap \mathcal{T}}$ con lo cual, aplicando el Lema 1.1.12, resulta que $P_{\mathcal{S}}$ y $P_{\mathcal{T}}$ conmutan.

A continuación estableceremos algunas relaciones entre ángulos y proyecciones. 
Lema 1.1.17. Sean $\mathcal{S}$ y $\mathcal{T}$ dos subespacios cerrados de un espacio de Hilbert $\mathcal{H}$. Entonces,

1. $c_{0}(\mathcal{S}, \mathcal{T})=0$ si y sólo si $\mathcal{S} \perp \mathcal{T}$.

2. $c(\mathcal{S}, \mathcal{T})=0$ si y sólo si $P_{\mathcal{S}}$ y $P_{\mathcal{T}}$ conmutan.

El próximo es un resultado clásico sobre ángulos, el cual utilizaremos en varias oportunidades a lo largo de este trabajo. El mismo puede encontrarse en [26, Teorema 13] ó, aún en una versión más general, en [49, Teorema 4.8].

Proposición 1.1.18. Las siguientes condiciones son equivalentes:

1. $c(\mathcal{S}, \mathcal{T})<1$,

2. $\mathcal{S}+\mathcal{T}$ es cerrado,

3. $\mathcal{S}^{\perp}+\mathcal{T}^{\perp}$ es cerrado,

4. $c\left(\mathcal{S}^{\perp}, \mathcal{T}^{\perp}\right)<1$,

5. $P_{\mathcal{S}^{\perp}}(\mathcal{T})$ es cerrado.

\subsubsection{Inversas generalizadas}

Definición. Dados $A \in L(\mathcal{H}, \mathcal{K})$ y $B \in L(\mathcal{K}, \mathcal{H})$, decimos que $B$ es una inversa generalizada de $A$ si

$$
A B A=A \quad \text { y } \quad B A B=B .
$$

$\mathrm{Al}$ conjunto de inversas generalizadas de $A$ lo denotaremos por medio de $\mathcal{G}(A)$.

Teorema 1.1.19. Sea $A \in L(\mathcal{H}, \mathcal{K})$.

1. Si $B \in \mathcal{G}(A)$, entonces $A B$ es una proyección (oblicua) con $R(A B)=R(A)$ y $B A$ es una proyección (oblicua) con $N(B A)=N(A)$.

2. A tiene rango cerrado si y sólo si $\mathcal{G}(A) \neq \varnothing$.

3. En tal caso, para cada par de proyecciones oblicuas $P \in L(\mathcal{K})$ y $Q \in L(\mathcal{H})$ tales que $R(P)=R(A)$ y $R(Q)=N(A)$, existe un único $B \in \mathcal{G}(A)$ tal que $A B=P$ y $B A=I-Q$.

Demostración. 1. Si $B \in \mathcal{G}(A)$ entonces,

$$
(B A)^{2}=B A B A=B A \quad \text { y } \quad(A B)^{2}=A B A B=A B .
$$

Además, $R(A B) \subseteq R(A)=R(A B A) \subseteq R(A B)$ y $N(A) \subseteq N(B A) \subseteq N(A B A)=N(A)$. Por lo tanto, $R(A B)=R(A)$ y $N(B A)=N(A)$.

3. Supongamos que $R(A)$ es cerrado, y sean $P \in L(\mathcal{K})$ y $Q \in L(\mathcal{H})$ proyecciones oblicuas tales que $R(P)=R(A)$ y $R(Q)=N(A)$. Llamemos $\mathcal{S}=N(P)$ y $\mathcal{T}=N(Q)$. Luego, $N(A) \dot{+} \mathcal{T}=\mathcal{H}$ y en consecuencia $\left.A\right|_{\mathcal{T}}: \mathcal{T} \rightarrow R(A)$ es inversible. Llamemos $B_{0}: R(A) \rightarrow \mathcal{T}$ a su inversa, y sea

$$
B: \mathcal{K}=\mathcal{S}+R(A) \rightarrow \mathcal{H} \quad \text { definido por } \quad B(x+y)=B_{0} y, \quad \text { siendo } x \in \mathcal{S}, y \in R(A) .
$$


Como $B_{0}$ es acotado, aplicando el Teorema de la Aplicación Abierta se deduce que $B \in L(\mathcal{K}, \mathcal{H})$. En efecto, $\|B\| \leq\left\|B_{0}\right\|\|P\|$, ya que $B(z)=B_{0}(P z)$ para todo $z \in \mathcal{K}$. Finalmente, es fácil probar que $B \in \mathcal{G}(A), A B=P$ y $B A=I-Q$.

2. Si $\mathcal{G}(A) \neq \varnothing$ entonces $R(A)=R(A B)$ para toda $B \in \mathcal{G}(A)$. Luego, $R(A)$ es cerrado porque es el rango de una proyección. La recíproca se deduce del item 3, aplicado a las proyecciones $P=P_{R(A)}$ y $Q=P_{N(A)}$.

Definición. Dado $A \in L(\mathcal{H}, \mathcal{K})$ con rango cerrado, llamaremos inversa de Moore-Penrose de $A$ (y lo anotaremos $A^{\dagger}$ ) al único elemento de $\mathcal{G}(A)$ tal que $A^{\dagger} A$ y $A A^{\dagger}$ son proyecciones autoadjuntas.

Corolario 1.1.20. Dado $A \in L(\mathcal{H}, \mathcal{K}), R(A)$ es cerrado si y sólo si $R\left(A^{*}\right)$ es cerrado. Más aún, $\mathcal{G}\left(A^{*}\right)=\left\{B^{*}: B \in \mathcal{G}(A)\right\}$. En particular, $\left(A^{*}\right)^{\dagger}=\left(A^{\dagger}\right)^{*}$.

Demostración. La igualdad $\mathcal{G}\left(A^{*}\right)=\left\{B^{*}: B \in \mathcal{G}(A)\right\}$ se deduce fácilmente de la definición de inversa generalizada. Luego, la primera parte del corolario es consecuencia del Teorema 1.1.19. Para probar la última afirmación, notemos que $\left(A^{\dagger}\right)^{*}$ verifica las condiciones de la definición de $\left(A^{*}\right)^{\dagger}$.

Corolario 1.1.21. Sea $A \in L(\mathcal{H}, \mathcal{K})$ un operador con rango cerrado, $P \in L(\mathcal{K})$ una proyección con rango $R(A)$ y $Q \in L(\mathcal{H})$ un proyección con rango $N(A)$. Entonces, $B=(I-Q) A^{\dagger} P$ es la única inversa generalizada de $A$ tal que $A B=P$ y $B A=I-Q$.

Demostración. Notemos que $A Q=0$ ya que $R(Q)=N(A)$. Luego,

$$
\begin{aligned}
& B A=(I-Q) A^{\dagger} P A=(I-Q) A^{\dagger} A=(I-Q) P_{N(A)^{\perp}}=(I-Q)\left(P_{N(A) \perp}+P_{N(A)}\right)=I-Q, \\
& A B=A(I-Q) A^{\dagger} P=A A^{\dagger} P=P_{R(A)} P=P .
\end{aligned}
$$

Finalmente, supongamos que existe otra inversa generalizada $C$ de $A$ tal que $A C=P$ y $C A=$ $I-Q$. Luego,

$$
C=(C A) C=(I-Q) C=(B A) C=B(A C)=B P=B(A B)=B .
$$

\section{Ejemplos 1.1.22.}

1. Si $A \in G L(\mathcal{H})$, entonces $\mathcal{G}(A)=\left\{A^{-1}\right\}$. En particular, $A^{\dagger}=A^{-1}$.

2. Si $V \in L(\mathcal{H}, \mathcal{K})$ es una isometría parcial entonces $V^{\dagger}=V^{*}$.

3. Si $A$ es normal y $R(A)$ es cerrado, entonces $A$ conmuta con $A^{\dagger}$.

4. Si $A \in L(\mathcal{H}, \mathcal{K})$ tiene $R(A)$ cerrado y los operadores $U \in L(\mathcal{K})$ y $W \in L(\mathcal{H})$ son unitarios, entonces $(U A W)^{\dagger}=W^{*} A^{\dagger} U^{*}$.

Definición. Un operador $A \in L(\mathcal{H})$ se dice acotado inferiormente si existe un $\alpha>0$ tal que $\|A x\| \geq \alpha\|x\|$ para todo $x \in N(A)^{\perp}$.

Proposición 1.1.23. Si $A \in L(\mathcal{H})$ entonces, $A$ es acotado inferiormente si y sólo si $R(A)$ es cerrado. 
Demostración. Sea $A \in L(\mathcal{H})$ y supongamos que $A$ es acotado inferiormente. Si $y \in \overline{R(A)}$, existe una sucesión $\left\{x_{n}\right\}_{n \in \mathbb{N}}$ en $N(A)^{\perp}$ tal que $A x_{n} \rightarrow y$. Luego, dado $\varepsilon>0$, existe un $n_{0} \in \mathbb{N}$ tal que $\left\|x_{n}\right\| \leq \frac{1}{\alpha}\left\|A x_{n}\right\| \leq \frac{1}{\alpha}(\|y\|+\varepsilon)$ para todo $n \geq n_{0}$. Por lo tanto, existe $M>0$ tal que $\left\|x_{n}\right\| \leq M$ para todo $n \in \mathbb{N}$.

Entonces, existe una subsucesión $\left\{x_{n_{k}}\right\}_{k \in \mathbb{N}}$ de $\left\{x_{n}\right\}_{n \in \mathbb{N}}$ tal que $x_{n_{k}} \rightarrow x_{0}$ para algún vector $x_{0} \in \mathcal{H}$. Luego, por la unicidad del límite, tenemos que $y=A x_{0}$. Por lo tanto, $R(A)$ es cerrado.

Recíprocamente, supongamos que $R(A)$ es cerrado. Luego, dado $x \in N(A)^{\perp}$,

$$
\|x\|=\left\|A^{\dagger} A x\right\| \leq\left\|A^{\dagger}\right\|\|A x\| .
$$

Por lo tanto, eligiendo $\alpha=\left\|A^{\dagger}\right\|^{-1}>0$, tenemos que $\|A x\| \geq \alpha\|x\|$ para todo $x \in N(A)^{\perp}$.

\subsection{Espacios vectoriales con métrica indefinida}

En esta sección presentaremos algunos resultados básicos sobre espacios con métrica indefinida, prestando una destacada atención a los espacios de Krein. Para una exposición más amplia sobre los temas aquí desarrollados sugerimos al lector los libros de J. Bognár [9] y T. Ya. Azizov \& I. S. Iokhvidov [43], las monografías redactadas por T. Ando [4] y por M. Dritschel \& J. Rovnyak [29] y el trabajo de J. Rovnyak [65].

Definición. Un espacio con métrica indefinida es un $\mathbb{C}$-espacio vectorial $\mathcal{V}$ dotado de una aplicación $[]:, \mathcal{V} \times \mathcal{V} \rightarrow \mathbb{C}$ que satisface los siguientes axiomas:

1. $[\alpha x+\beta y, z]=\alpha[x, z]+\beta[y, z]$ para todo $x, y, z \in \mathcal{V}$ y $\alpha, \beta \in \mathbb{C}$

2. $[x, y]=\overline{[y, x]}$ para todo $x, y \in \mathcal{V}$.

El antiespacio $-\mathcal{V}$ de un espacio con métrica indefinida $(\mathcal{V},[]$,$) es el espacio (\mathcal{V},-[]$,$) . Por$ lo tanto, $[,]_{-\mathcal{V}}=-[,]_{\mathcal{V}}$.

Dado un espacio con métrica indefinida $\mathcal{V}$, utilizamos las nociones de subespacio, ortogonalidad y suma directa de la misma forma que para espacios de Hilbert. Por ejemplo, $x, y \in \mathcal{V}$ se dicen ortogonales respecto a la métrica indefinida si $[x, y]=0$. Lo anotaremos $x[\perp] y$. De la misma manera, diremos que $x \in \mathcal{V}$ es ortogonal a un conjunto $\mathcal{M}$ (y lo anotaremos $x[\perp] \mathcal{M}$ ) si $[x, y]=0$ para todo $y \in \mathcal{M}$. Dado $\mathcal{M} \subset \mathcal{V}$, el subespacio ortogonal a $\mathcal{M}$ respecto a la métrica indefinida es el conjunto

$$
\mathcal{M}^{[\perp]}=\{x \in \mathcal{V}: x[\perp] \mathcal{M}\} .
$$

Proposición 1.2.1. Sean $\mathcal{M}$ y $\mathcal{N}$ subconjuntos de $\mathcal{V}$. El complemento ortogonal es siempre un subespacio y cumple:

1. si $\mathcal{M} \subset \mathcal{N}$ entonces $\mathcal{N}^{[\perp]} \subset \mathcal{M}^{[\perp]}$;

2. $(\mathcal{M}+\mathcal{N})^{[\perp]} \subseteq \mathcal{M}^{[\perp]} \cap \mathcal{N}^{[\perp]}$;

3. $\mathcal{M}^{[\perp]}+\mathcal{N}^{[\perp]} \subseteq(\mathcal{M} \cap \mathcal{N})^{[\perp]}$;

4. $\mathcal{M} \subseteq \mathcal{M}^{[\perp][\perp]}$;

5. si $\mathcal{M}$ y $\mathcal{N}$ son subespacios, $(\mathcal{M}+\mathcal{N})^{[\perp]}=\mathcal{M}^{[\perp]} \cap \mathcal{N}^{[\perp]}$. 
Dado un subespacio $\mathcal{S}$ de $\mathcal{V}$, llamaremos parte isotrópica de $\mathcal{S}$ al subespacio $\mathcal{S}^{\circ}=\mathcal{S} \cap \mathcal{S}^{[\perp]}$ y diremos que $\mathcal{S}$ es no degenerado si $\mathcal{S}^{\circ}=\{0\}$.

Un vector $x \in \mathcal{V}$ se dice positivo, negativo ó neutro de acuerdo con el signo de la constante real $[x, x]$, es decir, si $[x, x]>0,[x, x]<0$ ó $[x, x]=0$, respectivamente. Un subespacio $\mathcal{S} \subset \mathcal{V}$ se dice positivo si los vectores no nulos de $\mathcal{S}$ son positivos. De la misma manera se definen subespacios negativos, neutros, no negativos y no positivos. $\mathcal{S}$ se dice definido si es positivo o negativo, es semidefinido si es no negativo o no positivo, y es indefinido en caso contrario.

Observación 1.2.2. Si $x \in \mathcal{V}$ es un vector positivo entonces $\alpha x$ también es positivo para todo $\alpha \in \mathbb{C}, \alpha \neq 0$. Sin embargo, la suma de dos vectores positivos puede no ser positiva. Por ejemplo, consideremos el $\mathbb{C}$-espacio vectorial $\mathcal{V}=\mathbb{C}^{2}$ dotado con la métrica indefinida dada por

$$
[x, y]=x_{1} \overline{y_{1}}-x_{2} \overline{y_{2}}, \quad \text { para } x=\left(x_{1}, x_{2}\right), y=\left(y_{1}, y_{2}\right) \in \mathcal{V} .
$$

Dados $x=(1,1 / 2), y=(-1,1 / 2) \in \mathbb{C}^{2}, x$ e $y$ son vectores positivos en esta métrica, pero

$$
[x+y, x+y]=[(0,1),(0,1)]=-1<0 .
$$

Proposición 1.2.3. Si $\mathcal{V}$ es indefinido entonces $\mathcal{V}$ contiene vectores neutros distintos de 0.

Demostración. Sean $x, y \in \mathcal{V}$ tales que $[x, x]>0$ y $[y, y]<0$. Definamos

$$
\varphi(t)=[(1-t) x+t y,(1-t) x+t y], \quad t \in[0,1] .
$$

Como $\varphi$ es continua en $[0,1], \varphi(0)>0$ y $\varphi(1)<0$, por del Teorema del Valor Medio sabemos que existe $0<t_{0}<1$ tal que $\varphi\left(t_{0}\right)=0$. Luego, $x_{0}=\left(1-t_{0}\right) x+t_{0} y \in \mathcal{V}$ es neutro y $x_{0} \neq 0$ ya que, como consecuencia de la Observación 1.2.2, $x$ e $y$ son linealmente independientes.

Proposición 1.2.4 (Desigualdad de Cauchy-Bunyakowski-Schwarz). Si S es un subespacio semidefinido de $\mathcal{V}$ entonces

$$
|[x, y]|^{2} \leq[x, x][y, y], \quad x, y \in \mathcal{S}
$$

Demostración. Supongamos que $\mathcal{S}$ es no negativo y, dados $x, y \in \mathcal{V}$, consideremos la forma sesquilineal semidefinida positiva $\varphi: \mathbb{C} \times \mathbb{C} \rightarrow \mathbb{C}$ dada por

$$
\varphi(\alpha, \beta)=[\alpha x+\beta y, \alpha x+\beta y]=\left(\begin{array}{ll}
\bar{\alpha} & \bar{\beta}
\end{array}\right)\left(\begin{array}{ll}
{[x, x]} & {[x, y]} \\
{[y, x]} & {[y, y]}
\end{array}\right)\left(\begin{array}{l}
\alpha \\
\beta
\end{array}\right) \quad \alpha, \beta \in \mathbb{C} .
$$

Luego, $0 \leq \operatorname{det}\left(\begin{array}{cc}{[x, x]} & {[x, y]} \\ {[y, x]} & {[y, y]}\end{array}\right)=[x, x][y, y]-|[x, y]|^{2}$.

Corolario 1.2.5. Si $S$ es semidefinido entonces $S^{\circ}=\{x \in S:[x, x]=0\}$.

Demostración. Si $z \in \mathcal{S}, z \neq 0$ satisface $[z, z]=0$ entonces, por Cauchy-Bunyakowsky-Schwarz,

$$
|[x, z]|^{2} \leq[x, x][z, z]=0, \quad \text { para todo } x \in \mathcal{S} .
$$

es decir, $z \in \mathcal{S}^{\circ}$. La otra inclusión es trivial. 
Si $\mathcal{S}$ es un subespacio definido entonces $\mathcal{S}$ es no degenerado. No obstante, también existen subespacios indefinidos y no degenerados.

Ejemplo 1.2.6. Sea $\mathcal{V}$ el espacio de sucesiones de números complejos con una cantidad finita de coordenadas no nulas, dotado de la métrica indefinida dada por

$$
[x, y]=-x_{1} y_{1}+\sum_{n=1}^{\infty} x_{n} \overline{y_{n}} \quad \text { para } x=\left(x_{n}\right)_{n \in \mathbb{N}}, y=\left(y_{n}\right)_{n \in \mathbb{N}} \in \mathcal{V}
$$

Si $S=\operatorname{span}\left\{e_{1}, e_{2}\right\}$ entonces $S$ es un subespacio indefinido (pues $e_{1}$ es negativo y $e_{2}$ es positivo). Además, $\mathcal{S}$ es no degenerado. De hecho, si $x=\left(x_{n}\right)_{n \in \mathbb{N}} \in \mathcal{S}^{[\perp]}$, tenemos que $\left[x, e_{1}\right]=\left[x, e_{2}\right]=0$ y esto implica que $\mathcal{S}^{[\perp]} \subseteq \operatorname{span}\left\{e_{k}: k \geq 3\right\}$. Luego, $\mathcal{S} \cap \mathcal{S}^{[\perp]}=\{0\}$.

\subsubsection{Subespacios descomponibles}

Consideremos un subespacio degenerado arbitrario $\mathcal{S}$ de un espacio con métrica indefinida $\mathcal{V}$ y su parte isotrópica $\mathcal{S}^{\circ}$. Es bien sabido que existe una cantidad infinita de descomposiciones de $\mathcal{S}$ en una suma directa de la forma

$$
\mathcal{S}=\mathcal{S}^{\circ}+\mathcal{S}^{\prime},
$$

donde $\mathcal{S}^{\prime}$ es cualquier complemento algebraico de $\mathcal{S}^{\circ}$. Notemos que, como $\mathcal{S}^{\circ} \cap \mathcal{S}^{\prime}=\{0\}$ y todos los vectores isotrópicos para $\mathcal{S}$ están contenidos en $\mathcal{S}^{\circ}$, para cualquier elección posible, el complemento $\mathcal{S}^{\prime}$ es un subespacio no degenerado.

Además, una descomposición como la de (1.2.1) plantea naturalmente preguntas acerca de la posibilidad de descomponer al subespacio no degenerado $\mathcal{S}^{\prime}$, si este fuera indefinido, en suma directa de un subespacio positivo y otro negativo. Sin profundizar demasiado en este difícil problema, por el momento presentaremos algunos hechos simples relacionados con el tema.

En primer lugar, a pesar de que no es cierto que todo subespacio no degenerado $\mathcal{S}$ puede descomponerse en una suma directa $\mathcal{S}=\mathcal{S}^{+} \dot{+} \mathcal{S}^{-}$, con $\mathcal{S}^{+}$un subespacio positivo y $\mathcal{S}^{-}$un subespacio negativo (ver el Ejemplo 1.2.11 más adelante), la recíporca si es cierta:

Proposición 1.2.7. Si un subespacio $\mathcal{S}$ de $\mathcal{V}$ admite una descomposición

$$
\mathcal{S}=\mathcal{S}^{+} \dot{+} \mathcal{S}^{-}
$$

en suma directa de un subespacio positivo $\mathcal{S}^{+}$y un subespacio negativo $\mathcal{S}^{-}$, entonces $\mathcal{S}$ es no degenerado.

Demostración. Supongamos que $x_{0} \in \mathcal{S}^{0}$ y descompongámoslo de acuerdo con (1.2.2) en $x_{0}=$ $x_{0}^{+}+x_{0}^{-}$, con $x_{0}^{ \pm} \in \mathcal{S}^{ \pm}$. Luego,

$$
\begin{aligned}
& 0=\left[x_{0}, x_{0}^{+}\right]=\left[x_{0}^{+}, x_{0}^{+}\right]+\left[x_{0}^{-}, x_{0}^{+}\right] \quad \Rightarrow\left[x_{0}^{+}, x_{0}^{+}\right]=-\left[x_{0}^{-}, x_{0}^{+}\right], \\
& 0=\left[x_{0}, x_{0}^{-}\right]=\left[x_{0}^{+}, x_{0}^{-}\right]+\left[x_{0}^{-}, x_{0}^{-}\right] \quad \Rightarrow\left[x_{0}^{-}, x_{0}^{-}\right]=-\left[x_{0}^{+}, x_{0}^{-}\right] .
\end{aligned}
$$

Entonces, es claro que $\left[x_{0}^{+}, x_{0}^{-}\right]$es un número real, es decir, $\left[x_{0}^{+}, x_{0}^{-}\right]=\left[x_{0}^{-}, x_{0}^{+}\right]$, y además $0 \leq\left[x_{0}^{+}, x_{0}^{+}\right]=\left[x_{0}^{-}, x_{0}^{-}\right] \leq 0$. Pero esto sólo es posible si $x_{0}^{+}=x_{0}^{-}=0$, i.e. $x_{0}=0$. 
Definición 1.2.8. Un subespacio $\mathcal{S}$ de un espacio con métrica indefinida $\mathcal{V}$ se dice descomponible si puede representarse como la suma directa ortogonal (con respecto a la métrica indefinida $[$,$] )$ de un subespacio neutro $\mathcal{S}^{\circ}$, un subespacio positivo $\mathcal{S}^{+}$y un subespacio negativo $\mathcal{S}^{-}$:

$$
\mathcal{S}=\mathcal{S}^{\circ}+\mathcal{S}^{+}+\mathcal{S}^{-}
$$

En primer lugar, justificaremos el uso del símbolo $\mathcal{S}^{\circ}$ para la componente neutra.

Lema 1.2.9. Dado un subespacio descomponible $\mathcal{S}$ de $\mathcal{V}$, si los subespacios $\mathcal{S}^{\circ}, \mathcal{S}^{+}$y $\mathcal{S}^{-}$satisfacen la Definición 1.2.8 entonces $\mathcal{S}^{\circ}$ coincide con la parte isotrópica de $\mathcal{S}$.

Demostración. Este resultado es una consecuencia de dos propiedades de la parte isotrópica:

1. Si $\mathcal{S}_{1}[\perp] \mathcal{S}_{2}$ y $\mathcal{S}=\mathcal{S}+\mathcal{S}_{2}$ entonces $\mathcal{S}^{\circ}=\mathcal{S}_{1}^{\circ}+\mathcal{S}_{2}^{\circ}$

2. Si $\mathcal{S}$ es un subespacio semidefinido entonces $\mathcal{S}^{\circ}$ coincide con el conjunto de vectores neutros de $\mathcal{S}$.

Corolario 1.2.10. Si $\mathcal{S}$ es un subespacio (descomponible) no degenerado de $\mathcal{V}$, toda descomposición de $\mathcal{S}$ es de la forma:

$$
\mathcal{S}=\mathcal{S}^{+} \dot{+} \mathcal{S}^{-},
$$

siendo $\mathcal{S}^{+}$un subespacio positivo y $\mathcal{S}^{-}$un subespacio negativo.

Ejemplo 1.2.11. Sea $\mathcal{V}$ el espacio vectorial formado por las sucesiones de numéros complejos $x=\left\{\xi_{j}\right\}_{j \in \mathbb{Z}}$ tales que $\xi_{j}=0$ para $j \leq j_{0}(x)$. Definamos una métrica indefinida sobre $\mathcal{V}$ mediante

$$
[x, y]=\sum_{j \in \mathbb{Z}} \xi_{j} \bar{\eta}_{-j-1},
$$

siendo $y=\left\{\eta_{j}\right\}_{j \in \mathbb{Z}} \in \mathcal{V}$.

Es fácil ver que $\mathcal{V}$ dotado con esta métrica indefinida es un espacio no degenerado. Por lo tanto, por el Corolario 1.2.10, si $\mathcal{V}$ fuera descomponible, toda descomposición de $\mathcal{V}$ debería ser de la forma

$$
\mathcal{V}=\mathcal{V}^{+} \dot{+} \mathcal{V}^{-}
$$

siendo $\mathcal{V}^{+}$un subespacio positivo de $\mathcal{V}$ y $\mathcal{V}^{-}$un subespacio negativo de $\mathcal{V}$.

Consideremos el operador lineal $T: \mathcal{V} \rightarrow \mathcal{V}$ definido por

$$
T\left(\left\{\xi_{j}\right\}_{j \in \mathbb{Z}}\right)=\left\{\ldots, \xi_{-2}, \xi_{-1}, 0,0,0, \ldots\right\} .
$$

Notemos que la restricción de $T$ a cualquier subespacio definido de $\mathcal{V}$ es inyectiva, ya que, al estar restringiéndonos a un subespacio definido, $T\left(\left\{\xi_{j}\right\}_{j \in \mathbb{Z}}\right)=0$ implica que $\left[\left\{\xi_{j}\right\}_{j \in \mathbb{Z}},\left\{\xi_{j}\right\}_{j \in \mathbb{Z}}\right]=0$, o equivalentemente, $\left\{\xi_{j}\right\}_{j \in \mathbb{Z}}=0$.

Por lo tanto, todo subespacio definido de $\mathcal{V}$ es isomorfo a alguna parte del subespacio de $\mathcal{V}$ formado por los $x=\left\{\xi_{j}\right\}_{j \in \mathbb{Z}}$ tales que $\xi_{j}=0$ para todo $j \geq 0$. En particular, si $\mathcal{V}=\mathcal{V}^{+} \dot{+} \mathcal{V}^{-}$ (siendo $\mathcal{V}^{+}$un subespacio positivo de $\mathcal{V}$ y $\mathcal{V}^{-}$un subespacio negativo de $\mathcal{V}$ ), entonces $\mathcal{V}^{+}$y $\mathcal{V}^{-}$ son isomorfos a ciertos subespacios del espacio vectorial de sucesiones finitas. Por otra parte, $\mathcal{V}$ es, por definición, isomorfo al espacio vectorial formado por todas las sucesiones infinitas. En consecuencia, llegamos un absurdo al suponer que $\mathcal{V}=\mathcal{V}^{+} \dot{+} \mathcal{V}^{-}$siendo $\mathcal{V}^{ \pm}$subespacios definidos de $\mathcal{V}$. 


\subsubsection{Espacios de Krein y descomposiciones canónicas}

La definición de espacio de Krein está formulada de manera tal que la clase de espacios de Krein contiene a los espacios de Hilbert, a los antiespacios de espacios de Hilbert, y es cerrada al considerar sumas directas ortogonales. Esta es la familia más pequeña de espacios con métrica indefinida con estas propiedades.

Definición. Un espacio de Krein es un espacio con métrica indefinida $(\mathcal{K},[]$,$) que puede$ descomponerse como una suma directa ortogonal (en el sentido indefinido)

$$
\mathcal{K}=\mathcal{K}_{+}+\mathcal{K}_{-},
$$

siendo $\left(\mathcal{K}_{+},[],\right)$un espacio de Hilbert y $\left(\mathcal{K}_{-},[],\right)$el antiespacio de un espacio de Hilbert. Una representación como esta se denomina descomposición canónica de $\mathcal{K}$.

Una descomposición canónica 1.2.5 de $\mathcal{K}$ permite definir un producto interno sobre todo el espacio de Krein $\mathcal{K}$ mediante la siguiente fórmula:

$$
\langle x, y\rangle=\left[x_{+}, y_{+}\right]-\left[x_{-}, y_{-}\right], \quad \text { siendo } x=x_{+}+x_{-}, y=y_{+}+y_{-} \in \mathcal{K}=\mathcal{K}_{+}+\mathcal{K}_{-} .
$$

Definición. Dado un espacio de Krein $\mathcal{K}$ con descomposición canónica (1.2.5), denotaremos $|\mathcal{K}|$ al espacio de Hilbert obtenido al reemplazar $\mathcal{K}_{-}$por su antiespacio $-\mathcal{K}_{-}$:

$$
|\mathcal{K}|=\mathcal{K}_{+} \oplus-\mathcal{K}_{-},
$$

donde la suma directa considerada es la suma directa ortogonal entre espacios de Hilbert. Notemos que $\mathcal{K}$ resulta un espacio de Banach, considerando la norma \|\|$=\|\|_{|\mathcal{K}|}$ del espacio de Hilbert $|\mathcal{K}|$.

Observación 1.2.12. Dado un espacio de Krein $\mathcal{K}$, cada descomposición canónica (1.2.5) de $\mathcal{K}$ también es ortogonal con respecto al producto interno del espacio de Hilbert asociado (1.2.6). De hecho, si $x_{+} \in \mathcal{K}_{+}$y $x_{-} \in \mathcal{K}_{-}$,

$$
\left\langle x_{+}, x_{-}\right\rangle_{|\mathcal{K}|}=\left[x_{+}, 0\right]_{\mathcal{K}}-\left[0, x_{-}\right]_{\mathcal{K}}=0
$$

Definición. Dado una descomposición canónica (1.2.5) de $\mathcal{K}$, el operador definido en $\mathcal{K}$ mediante

$$
J x=x_{+}-x_{-}, \quad \text { si } x=x_{+}+x_{-} \in \mathcal{K}, \quad x_{ \pm} \in \mathcal{K}_{ \pm},
$$

se denomina simetría fundamental u operador de signatura del espacio $\mathcal{K}$.

Notemos que la simetría $J$ puede interpretarse como un nexo entre la métrica indefinida del espacio de Krein $(\mathcal{K},[]$,$) y el producto interno del espacio de Hilbert (|\mathcal{K}|,\langle\rangle$,$) ya que, para$ cualquier par de vectores $x, y \in \mathcal{K}$,

$$
\langle x, y\rangle=[J x, y] \quad \text { y } \quad[x, y]=\langle J x, y\rangle .
$$

Luego, la métrica indefinida [, ] suele denominarse $J$-métrica. Cabe destacar que $J \in L(|\mathcal{K}|)$ y que $J=J^{*}=J^{-1}$ pues, dados $x=x_{+}+x_{-}, y=y_{+}+y_{-} \in \mathcal{K}$,

$$
\begin{aligned}
\langle J x, y\rangle & =\left\langle x_{+}-x_{-}, y_{+}+y_{-}\right\rangle=\left\langle x_{+}, y_{+}\right\rangle-\left\langle x_{-}, y_{-}\right\rangle=\left\langle x_{+}+x_{-}, y_{+}-y_{-}\right\rangle=\langle x, J y\rangle, \mathrm{y} \\
\langle J x, J y\rangle & =\left\langle x_{+}-x_{-}, y_{+}-y_{-}\right\rangle=\left\langle x_{+}, y_{+}\right\rangle+\left\langle x_{-}, y_{-}\right\rangle=\left\langle x_{+}-x_{-}, y_{+}-y_{-}\right\rangle=\langle x, y\rangle .
\end{aligned}
$$


Proposición 1.2.13 (Desigualdad de Cauchy-Bunyakowsky-Schwarz generalizada). La métrica indefinida $[$,$] es acotada respecto a \|\|$, más aún,

$$
|[x, y]| \leq\|x\|\|y\|, \quad x, y \in \mathcal{H}
$$

Demostración. Si $J$ es la simetría fundamental de $\mathcal{K}$ entonces, dados $x, y \in \mathcal{K}$,

$$
|[x, y]|^{2}=|\langle J x, y\rangle|^{2} \leq\|J\|\|x\|\|y\|=\|x\|\|y\| .
$$

Definición. Dos espacios con métrica indefinida $\mathcal{K}_{1}$ y $\mathcal{K}_{2}$ se dicen isomorfos si existe un isomorfismo (i.e. una biyección acotada) $T$ de $\mathcal{K}_{1}$ en $\mathcal{K}_{2}$ tal que

$$
[T x, T y]_{\mathcal{K}_{2}}=[x, y]_{\mathcal{K}_{1}}
$$

para todo $x, y \in \mathcal{K}_{1}$.

Un espacio de Krein (que no sea un espacio de Hilbert ni antiespacio de un Hilbert) tiene infinitas descomposiciones canónicas diferentes y, por lo tanto, infinitos espacios de Hilbert (y normas) asociados diferentes. Sin embargo, todas ellas son isomorfas.

Teorema 1.2.14. Sea $\mathcal{K}$ un espacio de Krein con dos descomposiciones canónicas

$$
\mathcal{K}=\mathcal{K}_{+} \oplus \mathcal{K}_{-} \quad \text { y } \mathcal{K}=\mathcal{K}_{+}^{\prime} \oplus \mathcal{K}_{-}^{\prime} .
$$

Sea $S \in L\left(\mathcal{K}_{+}, \mathcal{K}_{+}^{\prime}\right)$ definido por

$$
S x_{+}=x_{+}^{\prime}, \quad \text { si } x_{+} \in \mathcal{K}_{+} y \quad x_{+}=x_{+}^{\prime}+x_{-}^{\prime} \text { con } x_{ \pm}^{\prime} \in \mathcal{K}_{ \pm}^{\prime},
$$

y sea $T \in L\left(-\mathcal{K}_{-},-\mathcal{K}_{-}^{\prime}\right)$ dado por

$$
T y_{-}=y_{-}^{\prime}, \quad \text { si } y_{-} \in-\mathcal{K}_{-} y \quad y_{-}=y_{+}^{\prime}+y_{-}^{\prime} \operatorname{con} y_{ \pm}^{\prime} \in \mathcal{K}_{ \pm}^{\prime} .
$$

Luego, $S$ y $T$ son isomorfismos.

Demostración. Veamos que $S$ tiene gráfico cerrado. Consideremos una sucesión $\left(x_{n}, S x_{n}\right)_{n \in \mathbb{N}}$ en el gráfico de $S$ y vectores $x \in \mathcal{K}_{+}, y \in \mathcal{K}_{+}^{\prime}$ tales que $x_{n} \rightarrow x$ y $S x_{n} \rightarrow y$. Para cada $n \in \mathbb{N}$, tenemos que $w_{n}=x_{n}-S x_{n} \in\left(\mathcal{K}_{+}^{\prime}\right)^{[\perp]}=\mathcal{K}_{-}^{\prime}$. Luego, como para cada $z \in \mathcal{K}$ el funcional $\varphi_{z}: \mathcal{K}_{+}^{\prime} \rightarrow \mathbb{C}$ definido por $\varphi_{z}(x)=[x, z]$ es acotado, podemos asegurar que $x-y \in \mathcal{K}_{-}^{\prime}$. Entonces, la representación de $x$ de acuerdo a la descomposición $\mathcal{K}=\mathcal{K}_{+}^{\prime}+\mathcal{K}_{-}^{\prime}$ es $x=y+(x-y)$ y $y=S x$. Aplicando el Teorema del Gráfico Cerrado, vemos que $S \in L\left(\mathcal{K}_{+}, \mathcal{K}_{+}^{\prime}\right)$.

Supongamos que $x_{+} \in \mathcal{K}_{+}$y $x_{+}=x_{+}^{\prime}+x_{-}^{\prime}$ con $x_{ \pm}^{\prime} \in \mathcal{K}_{ \pm}^{\prime}$. Como

$$
\left\langle x_{+}, x_{+}\right\rangle_{\mathcal{K}_{+}}=\left[x_{+}, x_{+}\right]_{\mathcal{K}_{+}}=\left[x_{+}, x_{+}\right]_{\mathcal{K}}=\left[x_{+}^{\prime}, x_{+}^{\prime}\right]_{\mathcal{K}_{+}^{\prime}}+\left[x_{-}^{\prime}, x_{-}^{\prime}\right]_{\mathcal{K}_{-}^{\prime}} \leq\left[x_{+}^{\prime}, x_{+}^{\prime}\right]_{\mathcal{K}_{+}^{\prime}},
$$

$\left\|S x_{+}\right\|_{\mathcal{K}_{+}^{\prime}} \geq\left\|x_{+}\right\|_{\mathcal{K}_{+}}$. Por lo tanto, $S$ es inyectivo y tiene rango cerrado.

Pero además, el rango de $S$ coincide con $\mathcal{K}_{+}^{\prime}$. En efecto, supongamos que $z \in \mathcal{K}_{+}^{\prime}$ es ortogonal al rango de $S$ y sea $x_{+} \in \mathcal{K}_{+}$con descomposición $x_{+}=x_{+}^{\prime}+x_{-}^{\prime}, x_{ \pm}^{\prime} \in \mathcal{K}_{ \pm}^{\prime}$. Luego, $z[\perp] x_{+}^{\prime}$ $\left(x_{+}^{\prime}=S x_{+} \in R(S)\right)$ y $z[\perp] x_{-}^{\prime}\left(x_{-}^{\prime} \in \mathcal{K}_{-}^{\prime}=\left(\mathcal{K}_{+}^{\prime}\right)^{[\perp]}\right)$, es decir $z[\perp] x_{+}$. Entonces $z \in \mathcal{K}_{-} \mathrm{y}$

$$
0 \leq\langle z, z\rangle_{\mathcal{K}_{+}^{\prime}}=[z, z]_{\mathcal{K}_{+}^{\prime}}=[z, z]_{\mathcal{K}}=[z, z]_{\mathcal{K}_{-}} \leq 0
$$

Por lo tanto, $z=0$ y hemos probado que $S$ es suryectivo. Lo afirmado para $T$ puede deducirse de la misma manera (o puede aplicarse lo que hemos probado al antiespacio de $\mathcal{K}$ ). 
Corolario 1.2.15. Si $\mathcal{K}$ es un espacio de Krein, los números

$$
\operatorname{ind}_{ \pm} \mathcal{K}=\operatorname{dim} \mathcal{K}_{ \pm}
$$

no dependen de la elección de la descomposición (1.2.5).

Llamaremos índices (positivo y negativo, respectivamente) del espacio $\mathcal{K}$ a los naturales ind $_{ \pm} \mathcal{K}$ mencionados en el corolario anterior.

Definición. Un espacio de Pontryagin es un espacio de Krein $\mathcal{K}$ con ind_ $\mathcal{K}<\infty$.

Ejemplo 1.2.16. Definamos el espacio de Minkowski $\mathbb{M}^{n+1}$ como el conjunto de vectores $(n+1)$-dimensionales con entradas complejas. Dados dos elementos $a=\left(a_{1}, \ldots, a_{n}, \alpha\right)^{t} \mathrm{y}$ $b=\left(b_{1}, \ldots, b_{n}, \beta\right)^{t}$ definimos la métrica indefinida como

$$
[a, b]=a_{1} \overline{b_{1}}+\ldots+a_{n} \overline{b_{n}}-\alpha \bar{\beta}
$$

Luego, $\mathbb{M}^{n+1}$ es un espacio de Pontryagin con ind $+\mathbb{M}^{n+1}=n$ y ind $-\mathbb{M}^{n+1}=1$. Si representamos a los operadores sobre $\mathbb{M}^{n+1}$ como matrices (en la forma usual), entonces la simetría fundamental para el espacio está dada por

$$
J_{\mathbb{M}^{n+1}}=\left(\begin{array}{cc}
I_{n} & 0 \\
0 & -1
\end{array}\right),
$$

donde $I_{n}$ representa a la matriz identidad de orden $n$. Luego, $\left|\mathbb{M}^{n+1}\right|=\mathbb{C}^{n+1}$ con el producto interno canónico.

Corolario 1.2.17. Sea $\mathcal{K}$ un espacio de Krein con dos descomposiciones canónicas (1.2.8) y espacios de Hilbert asociados

$$
|\mathcal{K}|=\mathcal{K}_{+} \oplus-\mathcal{K}_{-} \quad y \quad\left|\mathcal{K}^{\prime}\right|=\mathcal{K}_{+}^{\prime} \oplus-\mathcal{K}_{-}^{\prime}
$$

Entonces, $|\mathcal{K}| y\left|\mathcal{K}^{\prime}\right|$ tienen normas equivalentes, es decir, existen $m>0$ y $M>0$ tales que

$$
m\|x\|_{|\mathcal{K}|} \leq\|x\|_{\left|\mathcal{K}^{\prime}\right|} \leq M\|x\|_{|\mathcal{K}|}
$$

para todo $x \in \mathcal{K}$. Por lo tanto, las topologías (de la norma) de los espacios de Hilbert (1.2.9) son idénticas.

Demostración. Representemos a un vector arbitrario $x \in \mathcal{K}$ como

$$
x=x_{+}+x_{-}=x_{+}^{\prime}+x_{-}^{\prime}
$$

con $x_{ \pm} \in \mathcal{K}_{ \pm}$y $x_{ \pm}^{\prime} \in \mathcal{K}_{ \pm}^{\prime}$. Si $S$ y $T$ son los operadores del teorema anterior, entonces $x_{+}=$ $S x_{+}+(I-S) x_{+}, x_{-}=(I-T) x_{-}+T x_{-} \operatorname{con} S x_{+},(I-T) x_{-} \in \mathcal{K}_{+}^{\prime}$ y $(I-S) x_{+}, T x_{-} \in \mathcal{K}_{-}^{\prime}$. Por lo tanto,

$$
\begin{aligned}
& x_{+}^{\prime}=S x_{+}+(I-T) x_{-}, \\
& x_{-}^{\prime}=(I-S) x_{+}+T x_{-} .
\end{aligned}
$$

De la misma manera pueden encontrarse otras relaciones parecidas invirtiendo los roles de las descomposiciones canónicas, obteniéndose así constante $m$ y $M$ adecuadas. 


\subsubsection{Operadores sobre espacios de Krein}

La geometría de los espacios de Krein tiene muchas similitudes con la de los espacios de Hilbert pero tiene también diferencias significativas como la existencia de subespacios negativos. Es conveniente estudiar la geometría de los espacios de Krein conjuntamente con algunas clases de operadores, incluyendo los operadores autoadjuntos, las proyecciones ortogonales y las isometrías. El objetivo de esta sección es introducir las principales clases de operadores y mostrar su relación con la geometría de espacios de Krein.

Definición. La topología de la norma de un espacio de Krein $\mathcal{K}$ es la topología inducida por la norma de cualquier espacio de Hilbert (1.2.6) asociado a una descomposición canónica (1.2.5) de $\mathcal{K}$.

Las nociones de convergencia y continuidad que aparezcan en el resto de esta sección corresponden a la topología de la norma, a menos que se haga explícito el uso de otra topología.

$\mathrm{Al}$ espacio de operadores acotados entre dos espacios de Krein $\mathcal{H}$ y $\mathcal{K}$ lo denotaremos $L(\mathcal{H}, \mathcal{K})$, de la misma forma que lo hacemos para espacios de Hilbert. Recordemos que si $\mathcal{H}=\mathcal{K}$ reemplazaremos $L(\mathcal{H}, \mathcal{H})$ por $L(\mathcal{H})$.

Notemos que, dado un espacio de Krein $\mathcal{K}$ y fijada una descomposición canónica de $\mathcal{K}$, si dotamos a $\mathcal{K}$ de la topología de la norma, este resulta - como espacio topológico - indistinguible de su espacio de Hilbert asociado $|\mathcal{K}|$. Entonces, si $\mathcal{H}$ y $\mathcal{K}$ son dos espacios de Krein y $A: \mathcal{H} \rightarrow \mathcal{K}$ es un operador lineal, vemos que $A \in L(\mathcal{H}, \mathcal{K})$ si y sólo si $A \in L(|\mathcal{H}|,|\mathcal{K}|)$.

Luego, $L(\mathcal{H}, \mathcal{K})$ coincide con $L(|\mathcal{H}|,|\mathcal{K}|)$ y decimos que la norma \|\| de $L(|\mathcal{H}|,|\mathcal{K}|)$ es una norma de operadores para $L(\mathcal{H}, \mathcal{K})$.

Proposición 1.2.18. Dados dos espacios de Krein $\mathcal{H}$ y $\mathcal{K}$, si dotamos al espacio $L(\mathcal{H}, \mathcal{K})$ con la norma

$$
\|A\|=\sup _{\|x\|_{|\mathcal{H}|=1}}\|A x\|_{|\mathcal{K}|}, \quad A \in L(\mathcal{H}, \mathcal{K})
$$

el espacio de operadores acotados resulta un espacio de Banach.

Observación 1.2.19. Todo par de normas de operadores para $L(\mathcal{H}, \mathcal{K})$ son equivalentes, por lo tanto tenemos una única topología (de la norma) para $L(\mathcal{H}, \mathcal{K})$. Las topologías fuerte de operadores $(\mathrm{SOT})$ y débil de operadores $(\mathrm{WOT})$ se definen para $L(\mathcal{H}, \mathcal{K})$ de la misma manera que para espacios de Hilbert.

Como consecuencia de la identificación de $L(\mathcal{H}, \mathcal{K})$ con $L(|\mathcal{H}|,|\mathcal{K}|)$, pueden enunciarse para espacios de Krein muchos de los resultados clásicos de teoría de operadores en espacios de Hilbert. Por ejemplo:

- El Teorema del Gráfico Cerrado: un operador lineal de un espacio de Krein $\mathcal{H}$ en un espacio de Krein $\mathcal{K}$, definido en todo $x \in \mathcal{H}$, es continuo si y sólo si tiene el gráfico cerrado.

- El Teorema de Representación de Riesz: todo funcional lineal continuo $\varphi$ sobre un espacio de Krein $\mathcal{K}$ es de la forma

$$
\varphi(x)=[x, y], \quad x \in \mathcal{K},
$$

para un único $y \in \mathcal{K}$. 
Para cada $A \in L(\mathcal{H}, \mathcal{K})$ existe un único operador $A^{\#} \in L(\mathcal{K}, \mathcal{H})$, llamado el $J$-adjunto de $A$, tal que

$$
[A x, y]_{\mathcal{K}}=\left[x, A^{\#} y\right]_{\mathcal{H}}, \quad x \in \mathcal{H}, y \in \mathcal{K} .
$$

La existencia del $J$-adjunto es consecuencia del Teorema de Representación de Riesz y sus propiedades elementales son inmediatas, al igual que en el caso de un espacio de Hilbert. Por ejemplo, cuando las operaciones estén definidas

$$
(A+B)^{\#}=A^{\#}+B^{\#}, \quad(A B)^{\#}=B^{\#} A^{\#} .
$$

Los conceptos de operador $J$-adjunto (respecto a la métrica indefinida del espacio de Krein $\mathcal{H}$ ) y adjunto (respecto al producto interno del espacio de Hilbert asociado $|\mathcal{H}|$ ) están íntimamente relacionados: si $A \in L(\mathcal{H}, \mathcal{K})$ entonces

$$
A^{\#}=J_{\mathcal{H}} A^{*} J_{\mathcal{K}}
$$

siendo $A^{*} \in L(|\mathcal{K}|,|\mathcal{H}|)$ el operador adjunto de $A$. En particular, $\left\|A^{\#}\right\|=\|A\|$ para todo $A \in L(\mathcal{H}, \mathcal{K})$ y cualquier norma de operadores sobre $L(\mathcal{H}, \mathcal{K})$.

La prueba de la identidad (1.2.10) es directa, ya que si $x \in \mathcal{H}$ e $y \in \mathcal{K}$ entonces $\left[x, A^{\#} y\right]_{\mathcal{H}}=$ $[A x, y]_{\mathcal{K}}=\left\langle J_{\mathcal{K}} A x, y\right\rangle_{|\mathcal{K}|}=\left\langle x, A^{*} J_{\mathcal{K}} y\right\rangle_{|\mathcal{H}|}=\left[x, J_{\mathcal{H}} A^{*} J_{\mathcal{K}} y\right]_{\mathcal{H}} \cdot$

Definición. Dado un espacio de Krein con simetría fundamental $J$, un operador $A \in L(\mathcal{H})$ es $J$-autoadjunto si $A^{\#}=A$.

Dentro del espacio vectorial (real) de operadores $J$-autoadjuntos podemos distinguir al cono de operadores $J$ - positivos: decimos que $A$ es $J$-positivo - y lo anotamos $A \geq_{J} 0$ - si

$$
[A x, x]_{\mathcal{H}} \geq 0, \quad x \in \mathcal{H} .
$$

Luego, los operadores $J$-autoadjuntos sobre un espacio de Krein $\mathcal{H}$ están parcialmente ordenados de la siguiente manera:

$$
A \leq_{J} B \text { si y sólo si } B-A \geq_{J} 0 .
$$

Por su parte, un operador $J$-autoadjunto $A$ se dice $J$-negativo (y se anota $A \leq_{J} 0$ ) si $-A$ es $J$-positivo.

Definición. Dados dos espacios de Krein $\mathcal{H}$ y $\mathcal{K}$, un operador $A \in L(\mathcal{H}, \mathcal{K})$ es:

1. J-isométrico si $A^{\#} A=I_{\mathcal{H}}$;

2. una $J$-isometría parcial si $A A^{\#} A=A$;

3. J-unitario si $A$ y $A^{\#}$ son isométricos.

Observación 1.2.20. Un operador $U \in L(\mathcal{H}, \mathcal{K})$ es $J$-unitario si y sólo si $U$ es un isomorfismo entre los espacios de Krein $\mathcal{H}$ y $\mathcal{K}$. 


\section{Factorización de Bognár-Kramli}

Dado un operador positivo $A \in L(\mathcal{H})$ actuando en un espacio de Hilbert $\mathcal{H}$, consideremos su raíz cuadrada, $A^{1 / 2}$. Notemos que el subespacio $\mathcal{K}=N(A)^{\perp}$ también es un espacio de Hilbert (con la topología inducida por $\mathcal{H}$ ), mientras que el operador $D \in L(\mathcal{K}, \mathcal{H})$ definido por

$$
D x=A^{1 / 2} x, \quad x \in \mathcal{K},
$$

resulta inyectivo y además cumple:

$$
A=D D^{*},
$$

siendo $D^{*} \in L(\mathcal{H}, \mathcal{K})$ es operador adjunto de $D$. El Teorema de Bognár-Kramli es una generalización de esta factorización, la cual se aplica a cualquier operador $J$-autoadjunto actuando en un espacio de Krein $\mathcal{K}$ (con simetría fundamental $J$ ).

Teorema 1.2.21 (Factorización de Bognár-Kramli). Sea $\mathcal{H}$ un espacio de Krein con simetría fundamental J. Si $A \in L(\mathcal{H})$ es un operador $J$-autoadjunto entonces puede factorizarse como

$$
A=D D^{\#}
$$

siendo $\mathcal{D}$ un espacio de Krein y $D \in L(\mathcal{D}, \mathcal{H})$ un operador inyectivo.

Demostración. Fijada la simetría fundamental $J$ de $\mathcal{H}$, sea $|\mathcal{H}|$ el espacio de Hilbert asociado. Como $A=A^{\#}=J A^{*} J, A J \in L(|\mathcal{H}|)$ es un operador autoadjunto y su descomposición polar es de la forma

$$
A J=P U=U P,
$$

siendo $P \in L(|\mathcal{H}|)^{+}$y $U$ una isometría parcial en $L(|\mathcal{H}|)$ con $\mathcal{D}=\overline{R(P)}$ como espacio inicial y final. Luego, $U$ también es autoadjunta y $\mathcal{D}$ es un espacio de Krein si lo dotamos con la simetría fundamental $J_{\mathcal{D}}=\left.U\right|_{\mathcal{D}}$. Sea $D: \mathcal{D} \rightarrow \mathcal{H}$ el operador lineal definido por

$$
D x=P^{1 / 2} x, \quad x \in \mathcal{D} .
$$

Como $P \in L(\mathcal{H})$ y $\mathcal{D}$ es un subespacio cerrado de $\mathcal{H}$, podemos afirmar que $D \in L(\mathcal{D}, \mathcal{H})$ y, por la Ec. (1.2.10), tenemos que $D^{\#}=U P^{1 / 2} J$. Entonces, $D D^{\#}=P^{1 / 2} U P^{1 / 2} J=(A J) J=A$. Notemos además que $N(D)=N(P) \cap \mathcal{D}=\{0\}$.

Observación 1.2.22. Como consecuencia de la demostración del Teorema de Bognár-Kramli tenemos que, si $A \geq_{J} 0$, podemos elegir el espacio vectorial $\mathcal{D}$ de manera tal que resulte un espacio de Hilbert.

De hecho, si $A \geq_{J} 0$ entonces $T=A J \in L(|\mathcal{H}|)^{+}$y podemos considerar el espacio vectorial $\mathcal{D}=\overline{R(T)}$ dotado con el producto interno de $|\mathcal{H}|$. Como $\mathcal{D}$ es un subespacio cerrado de $\mathcal{H}, \mathcal{D}$ es un espacio de Hilbert. Luego, definiendo $D=\left.T^{1 / 2}\right|_{\mathcal{K}} \in L(\mathcal{K}, \mathcal{H})$, es fácil ver que $N(D)=\{0\}$, $D^{\#}=T^{1 / 2} J$ y $D D^{\#}=T^{1 / 2} T^{1 / 2} J=T J=A$.

Definición. Dado un operador $J$-autoadjunto $T \in L(\mathcal{H})$, el índice positivo de $T$ (resp. índice negativo de $T$ ), al que denotaremos ind $T$ (resp. ind- $T$ ), es el supremo entre los $n \in \mathbb{N}$ tales que existe un subespacio $n$-dimensional de $\mathcal{H}$ que es un espacio de Hilbert (resp. antiespacio de un espacio de Hilbert) con la métrica indefinida

$$
[x, y]_{T}=[T x, y], \quad x, y \in \mathcal{H} .
$$

Si no existe un $n \in \mathbb{N}$ así, diremos que ind $+(T)=0$ (resp. ind_ $T=0)$. 
Equivalentemente, $\operatorname{ind}_{+}(T)$ es el supremo entre $\operatorname{los} n \in \mathbb{N}$ tales que existe una matriz definida positiva de la forma

$$
\left(\left[T x_{j}, x_{k}\right]\right)_{j, k=1, \ldots, n} \quad \operatorname{con} x_{1}, \ldots, x_{n} \in \mathcal{H} .
$$

Mientras que la factorización del Teorema 1.2.21 existe para todo operador acotado, en general ésta no es única (ni siquiera es única salvo isomorfismos). Sin embargo, los índices del espacio de Krein subyacente son únicos:

Teorema 1.2.23. Sea $\mathcal{H}$ un espacio de Krein con simetría fundamental $J$ y $T \in L(\mathcal{H})$ un operador J-autoadjunto. Consideremos una factorización $T=W W^{\#}$ con $W \in L(\mathcal{K}, \mathcal{H})$ para algún espacio de Krein $\mathcal{K}$ y $N(W)=\{0\}$ como en el Teorema 1.2.21. Entonces,

$$
\operatorname{ind}_{ \pm} \mathcal{K}=\operatorname{ind}_{ \pm} T \text {. }
$$

En particular, los indices ind $_{ \pm} \mathcal{K}$ no dependen de la factorización elegida.

La prueba del Teorema anterior la omitimos, puede encontrarse en [30, Teorema 1.2.1].

Definición. Un operador $J$-autoadjunto $T \in L(\mathcal{H})$ tiene la propiedad de factorización única (PFU) si dadas dos factorizaciones

$$
T=W_{i} W_{i}^{\#}, \quad \text { siendo } \mathcal{K}_{i} \text { un espacio de Krein y } W_{i} \in L\left(\mathcal{K}_{i}, \mathcal{H}\right) \text { inyectivo }(i=1,2),
$$

existe un isomorfismo $U \in L\left(\mathcal{K}_{1}, \mathcal{K}_{2}\right)$ tal que $W_{1}=W_{2} U$.

Observación 1.2.24. Sea $T \in L(\mathcal{H})$ es un operador $J$-autoadjunto con la PFU. Si $T=W_{i} W_{i}^{\#}$, $i=1,2$, son dos factorizaciones de $T$ como las del Teorema 1.2.21 entonces $R\left(W_{1}\right)=R\left(W_{2}\right)$.

De hecho, si $T$ tiene la PFU, consideremos dos factorizaciones de $T, T=W_{i} W_{i}^{\#}$ con $W_{i} \in$ $L\left(\mathcal{K}_{i}, \mathcal{H}\right)$ inyectivo, $i=1,2$. Si $U \in L\left(\mathcal{K}_{1}, \mathcal{K}_{2}\right)$ es un isomorfismo tal que $W_{1}=W_{2} U$, entonces $R\left(W_{1}\right)=R\left(W_{2} U\right)=R\left(W_{2}\right)$ ya que $R(U)=\mathcal{K}_{2}$.

En el siguiente teorema se detallan algunas condiciones suficientes para la propiedad de factorización única. La demostración puede encontrarse en [16, Teorema 2.8].

Teorema 1.2.25. Sea $\mathcal{H}$ un espacio de Krein con simetría fundamental $J$, y sea $T \in L(\mathcal{H})$ un operador J-autoadjunto. Cada una de las siguientes condiciones es suficiente para que $T$ tenga la propiedad de factorización única:

1. $T \geq_{J} 0$;

2. $\operatorname{ind}_{ \pm}(T)$ es finito;

3. $T^{2} \leq_{J} T$.

\subsubsection{Subespacios uniformemente definidos de un espacio de Krein}

Definición. sea $\mathcal{H}$ un espacio de Krein con norma \|\| . Un subespacio $\mathcal{S}$ de $\mathcal{H}$ es uniformemente positivo si existe un $\delta>0$ tal que

$$
[x, x] \geq \delta\|x\|^{2}, \quad \text { para todo } x \in \mathcal{S} .
$$

Análogamente, $\mathcal{S}$ es uniformemente negativo si existe un $\delta>0$ tal que

$$
[x, x] \leq-\delta\|x\|^{2}, \quad \text { para todo } x \in \mathcal{S} .
$$

Es importante destacar que estas nociones son independientes de la norma elegida. 
En los párrafos que siguen a continuación caracterizaremos a los subespacios uniformemente positivos. Los resultados equivalentes para subespacios uniformemente negativos pueden deducirse fácilmente.

Observación 1.2.26. Un subespacio cerrado $\mathcal{S}$ de un espacio de Krein $\mathcal{H}$ es uniformemente positivo si y sólo si es un espacio de Hilbert con la métrica indefinida de $\mathcal{H}$.

Supongamos que $(\mathcal{S},[]$,$) es un espacio de Hilbert, siendo [ , ] la métrica indefinida de \mathcal{H}$, y consideremos la norma asociada a este producto interno: $\||x|\|=[x, x]^{1 / 2}, x \in \mathcal{S}$.

Fijada una simetría fundamental $J$ de $\mathcal{H}$, sea $(|\mathcal{H}|,\langle\rangle$,$) el espacio de Hilbert asociado a \mathcal{H}$ y denotemos \|\| a su norma. Notemos que $(\mathcal{S},\|\|)$ es un espacio de Banach y además la aplicación identidad $i d:(\mathcal{S},\|\|) \rightarrow(\mathcal{S},\|\| \| \mid)$ es acotada, ya que

$$
\|\mid\| x\left\|^{2}=[x, x]=\langle J x, x\rangle \leq\right\| J\|\| x\left\|^{2}=\right\| x \|^{2}, \quad \text { si } x \in \mathcal{S} .
$$

Luego, por el Teorema de la Aplicación Abierta, sabemos que la inversa $i d^{\prime}:(\mathcal{S},\|\| \| \mid) \rightarrow(\mathcal{S},\|\|)$ también está acotada $\mathrm{y}$, por lo tanto, existe un $\alpha>0$ tal que $\|x\| \leq \alpha\|\| x\|\|$ para todo $x \in \mathcal{S}$. Entonces,

$$
[x, x]=\|\| x\|\|^{2} \geq \alpha^{2}\|x\|^{2}, \quad \text { si } x \in \mathcal{S},
$$

i.e. $\mathcal{S}$ es uniformemente positivo. Notemos que la recíproca es trivial.

Dado un espacio de Krein $\mathcal{H}$ y fijada una descomposición canónica $\mathcal{H}=\mathcal{H}_{+} \oplus \mathcal{H}_{-}$, identifiquemos - como espacios vectoriales - a $\mathcal{H}$ con $\mathcal{H}_{+} \times\left|\mathcal{H}_{-}\right|$, es decir, si $x=x_{+}+x_{-} \in \mathcal{H}$, con $x_{+} \in \mathcal{H}_{+}$y $x_{-} \in \mathcal{H}_{-}$identifiquemos a $x$ con el par $\left(x_{+}, x_{-}\right)^{t}$.

Si $\mathcal{S}$ un subespacio positivo de $\mathcal{H}$, notemos que $\mathcal{S}$ no puede contener elementos no triviales de la forma $(0, y)$ porque $\mathcal{S}$ es positivo. Esta condición garantiza que $\mathcal{S}=G r(K)$ para algún operador $K: \operatorname{Dom} K \subseteq \mathcal{H}_{+} \rightarrow R(K) \subseteq\left|\mathcal{H}_{-}\right|$.

Además, la $J$-positividad de $\mathcal{S}$ asegura que, para cada $x \in \operatorname{Dom} K,[x+K x, x+K x]_{\mathcal{H}} \geq 0$. Luego, si $x \in \operatorname{Dom} K$,

$$
0 \leq[x+K x, x+K x]_{\mathcal{H}}=[x, x]_{\mathcal{H}}+[K x, K x]_{\mathcal{H}}=\|x\|_{\mathcal{H}_{+}}-\|K x\|_{\left|\mathcal{H}_{-}\right|},
$$

y en consecuencia, $\|K x\|_{\left|\mathcal{H}_{-}\right|} \leq\|x\|_{\mathcal{H}_{+}}$. Por lo tanto, $\|K\| \leq 1$.

Definición (Representación de un subespacio definido como el gráfico de un operador). Dado un subespacio positivo $\mathcal{S}$ de un espacio de Krein $\mathcal{H}$, el operador angular de $\mathcal{S}$ es una contracción $K$ con $\operatorname{Dom} K \subseteq \mathcal{H}_{+}$y $R(K) \subseteq\left|\mathcal{H}_{-}\right|$tal que como subespacio de $\mathcal{H}_{+} \times\left|\mathcal{H}_{-}\right|$,

$$
\mathcal{S}=\operatorname{Gr}(K)=\left\{(x, K x)^{t}: x \in \operatorname{Dom} K\right\} .
$$

Un subespacio es maximal con respecto a alguna propiedad $(P)$ si éste verifica $(P)$ y no está propiamente incluído en ningún otro subespacio que la verifique. Por ejemplo, un subespacio positivo maximal es un subespacio $\mathcal{S}$ de $\mathcal{H}$ que no está propiamente contenido en otro subespacio positivo de $\mathcal{H}$.

Dado un subespacio positivo $\mathcal{S}$ de un espacio de Krein $\mathcal{H}$, representado por medio de su operador angular $K$, las siguientes afirmaciones son ciertas y no requieren demostración:

1. $\mathcal{S}$ es cerrado si y sólo si $\operatorname{Dom} K$ es cerrado en $\mathcal{H}_{+} ;$

2. $\mathcal{S}$ es uniformemente positivo si y sólo si $\|K\|<1$; 
3. $\mathcal{S}$ es positivo maximal si y sólo si $\operatorname{Dom} K=\mathcal{H}_{+}$;

4. $\mathcal{S}$ es uniformemente positivo maximal si y sólo si $\operatorname{Dom} K=\mathcal{H}_{+}$y $\|K\|<1$.

Teorema 1.2.27. Sea $\mathcal{H}=\mathcal{H}_{+} \oplus \mathcal{H}_{-}$una descomposición canónica de un espacio de Krein $\mathcal{H}$.

1. Un subespacio $\mathcal{S}$ de $\mathcal{H}$ es negativo maximal si y sólo si $\mathcal{S}^{[\perp]}$ es un subespacio positivo maximal de $\mathcal{H}$.

2. Si $\mathcal{S}_{+}$es un subespacio uniformemente positivo maximal de $\mathcal{H}$, entonces $\mathcal{S}_{-}=\mathcal{S}_{+}^{[\perp]}$ es uniformemente negativo maximal. Además, $\mathcal{H}=\mathcal{S}_{+} \oplus \mathcal{S}_{-}$es una descomposición canónica de $\mathcal{H}$.

3. Todo subespacio negativo de $\mathcal{H}$ está contenido en un subespacio negativo maximal de $\mathcal{H}$.

4. Todo subespacio uniformemente negativo de $\mathcal{H}$ está contenido en un subespacio uniformemente negativo maximal de $\mathcal{H}$.

5. Supongamos que $\mathcal{S}_{ \pm}$son subespacios uniformemente definidos de $\mathcal{H}$ tales que $\mathcal{S}_{+}[\perp] \mathcal{S}_{-}$. Entonces existe una descomposición canónica $\mathcal{H}=\mathcal{H}_{+} \oplus \mathcal{H}_{-}$tal que $\mathcal{S}_{ \pm} \subseteq \mathcal{H}_{ \pm}$.

Demostración. 2. Siguiendo la representación de un subespacio definido por medio de su operador angular y utilizando las afirmaciones anteriormente mencionadas, $\mathcal{S}_{+}$puede escribirse como

$$
\mathcal{S}_{+}=\left\{(u, K u): u \in \mathcal{H}_{+}\right\},
$$

siendo $K \in L\left(\mathcal{H}_{+},\left|\mathcal{H}_{-}\right|\right)$una contracción uniforme: $\|K\|<1$. Luego,

$$
\mathcal{S}_{-}=\left\{\left(K^{*} v, v\right): v \in\left|\mathcal{H}_{-}\right|\right\}
$$

En efecto, supongamos que $x \in \mathcal{S}_{+}^{[\perp]}$ y escribámoslo en términos de la descomposición canónica de $\mathcal{H}: x=x_{+}+x_{-}, x_{ \pm} \in \mathcal{H}_{ \pm}$. Entonces, $0=\left[u+K u, x_{+}+x_{-}\right]=\left[u, x_{+}\right]+\left[K u, x_{-}\right]$para todo $u \in \mathcal{H}_{+}$, o equivalentemente,

$$
\left\langle u, x_{+}\right\rangle_{\mathcal{H}_{+}}=\left[u, x_{+}\right]=-\left[K u, x_{-}\right]=\left\langle K u, x_{-}\right\rangle_{\left|\mathcal{H}_{-}\right|}=\left\langle u, K^{*} x_{-}\right\rangle_{\mathcal{H}_{+}} .
$$

Como $u \in \mathcal{H}_{+}$era arbitrario, tenemos que $x_{+}=K^{*} x_{-}$. Es decir, $\mathcal{S}_{-} \subseteq\left\{\left(K^{*} x_{-}, x_{-}\right): x_{-} \in\left|\mathcal{H}_{-}\right|\right\}$ y la otra inclusión es análoga. Además, dado que $K^{*} \in L\left(\left|\mathcal{H}_{-}\right|, \mathcal{H}_{+}\right)$cumple $\left\|K^{*}\right\|<1$, el subespacio $\mathcal{S}_{-}$es uniformemente negativo maximal.

Por la Observación 1.2.26, $\mathcal{S}_{+}$y $-\mathcal{S}_{-}$son espacios de Hilbert con la métrica indefinida de $\mathcal{H}$. Además, como $\left(\begin{array}{cc}I & K^{*} \\ K & I\end{array}\right) \in G L\left(\mathcal{H}_{+} \oplus\left|\mathcal{H}_{-}\right|\right)^{+}$, todo elemento de $\mathcal{H}_{+} \oplus\left|\mathcal{H}_{-}\right|$tiene una única representación de la forma

$$
(x, y)^{t}=\left(\begin{array}{cc}
I & K^{*} \\
K & I
\end{array}\right)\left(\begin{array}{c}
u \\
v
\end{array}\right)=(u, K u)^{t}+\left(K^{*} v, v\right)^{t}, \quad \text { con } u \in \mathcal{H}_{+} \mathrm{y} v \in\left|\mathcal{H}_{-}\right|
$$

Por lo tanto, $\mathcal{H}=\mathcal{S}_{+} \oplus \mathcal{S}_{-}$es una descomposición canónica de $\mathcal{H}$.

4. Sea $\mathcal{S}$ un subespacio uniformemente negativo de $\mathcal{H}$ y representémoslo como el gráfico del operador angular asociado $K$. Luego, $K \in L\left(\mathcal{D}, \mathcal{H}_{+}\right)$con $\mathcal{D}$ un subespacio cerrado de $\left|\mathcal{H}_{-}\right|$y 
$\|K\|<1$. Extendiendo $K$ a un operador $\widetilde{K} \in L\left(\left|\mathcal{H}_{-}\right|, \mathcal{H}_{+}\right)$con $\|\widetilde{K}\| \leq 1$, el gráfico de $\widetilde{K}, G r(\widetilde{K})$, es un subespacio uniformemente negativo maximal de $\mathcal{H}$ que contiene a $\mathcal{S}$.

5. Por 4., podemos extender $\mathcal{S}_{+}$a un subespacio uniformemente positivo maximal $\mathcal{M}_{+}$de $\mathcal{H}$. Ya que $\mathcal{S}_{+}$y $\mathcal{M}_{+}$son espacios de Hilbert con la métrica indefinida de $\mathcal{H}$, existe un espacio de Hilbert $\mathcal{T}_{+}$(con la métrica indefinida de $\mathcal{H}$ ) tal que

$$
\mathcal{M}_{+}=\mathcal{S}_{+} \oplus \mathcal{T}_{+}
$$

y la suma es ortogonal (respecto a la métrica indefinida de $\mathcal{H}$ ). Por $2 ., \mathcal{M}_{-}=\mathcal{M}_{+}^{[\perp]}$ es uniformemente negativo maximal y $\mathcal{H}=\mathcal{M}_{+} \oplus \mathcal{M}_{-}$es una descomposición canónica. Por lo tanto,

$$
\mathcal{H}=\left(\mathcal{S}_{+} \oplus \mathcal{T}_{+}\right) \oplus \mathcal{M}_{-}=\mathcal{S}_{+} \oplus\left(\mathcal{T}_{+} \oplus \mathcal{M}_{-}\right) .
$$

Es fácil ver además que $\mathcal{T}_{+} \oplus \mathcal{M}_{-}=\mathcal{S}_{+}^{[\perp]}$ es un espacio de Krein con la métrica indefinida de $\mathcal{H}$. Por otra parte, como $\mathcal{S}_{-}$es un subespacio uniformemente negativo de $\mathcal{S}_{+}^{[\perp]}$, podemos aplicar un argumento similar al anterior para probar que

$$
\mathcal{S}_{+}^{[\perp]}=\mathcal{S}_{-} \oplus\left(\mathcal{N}_{+} \oplus \mathcal{N}_{-}\right)
$$

siendo $\mathcal{N}_{+}$y $-\mathcal{N}_{-}$subespacios de $\mathcal{H}$ (que a su vez son espacios de Hilbert con la métrica indefinida de $\mathcal{H}$ ). Entonces,

$$
\mathcal{H}=\mathcal{S}_{+} \oplus \mathcal{S}_{+}^{[\perp]}=\left(\mathcal{S}_{+} \oplus \mathcal{N}_{+}\right) \oplus\left(\mathcal{S}_{-} \oplus \mathcal{N}_{-}\right),
$$

y la descomposición canónica obtenida es $\mathcal{H}_{ \pm}=\mathcal{S}_{ \pm} \oplus \mathcal{N}_{ \pm}$.

\subsubsection{Geometría en espacios de Krein}

Recordemos que, si $\mathcal{S}$ es un subespacio de un espacio de Krein $\mathcal{H}$, el subespacio J-ortogonal a $\mathcal{S}$ está definido por

$$
\mathcal{S}^{[\perp]}=\{y \in \mathcal{H}:[x, y]=0 \text { para todo } x \in \mathcal{H}\} .
$$

Además, por la Proposición 1.2.1, para cualquier par de subespacios $\mathcal{S}$ y $\mathcal{T}$ de $\mathcal{H}$, valen las siguientes relaciones:

$$
\mathcal{S}^{[\perp][\perp]}=\overline{\mathcal{S}}, \quad(\mathcal{S}+\mathcal{T})^{[\perp]}=\mathcal{S}^{[\perp]} \cap \mathcal{T}^{[\perp]}, \quad \mathcal{S}^{[\perp]}+\mathcal{T}^{[\perp]} \subseteq(\mathcal{S} \cap \mathcal{T})^{[\perp]},
$$

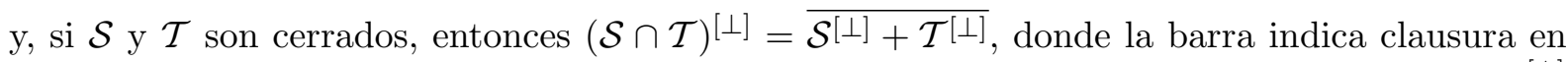
norma. Notemos que, si $J_{\mathcal{H}}$ es una simetría fundamental de $\mathcal{H}$, el subespacio $J$-ortogonal $\mathcal{S}^{[\perp]}$ está relacionado con el complemento ortogonal $\mathcal{S}^{\perp}($ de $\mathcal{S}$ en $|\mathcal{K}|)$ por las siguientes igualdades:

$$
\mathcal{S}^{[\perp]}=J\left(\mathcal{S}^{\perp}\right)=J(\mathcal{S})^{\perp} .
$$

No utilizamos el término "complemento $J$-ortogonal" para $\mathcal{S}^{[\perp]}$ porque el Teorema de la Proyección es falso en espacios de Krein, es decir, si $\mathcal{S}$ es un subespacio cerrado de $\mathcal{H}$, el subespacio $\mathcal{S} \oplus \mathcal{S}^{[\perp]}$ no coincide necesariamente con $\mathcal{H}$.

Una de las razones por la que puede fallar el Teorema de la Proyección es que el subespacio $\mathcal{S}$ sea $J$-degenerado, es decir, si $\mathcal{S}^{\circ}=\mathcal{S} \cap \mathcal{S}^{[\perp]} \neq\{0\}$. En este caso, la suma algebraica $\mathcal{S}+\mathcal{S}^{[\perp]}$ 
ni siquiera es una suma directa. Por ejemplo, si $\mathcal{S}=\operatorname{span}\{(1,1)\}$ en el espacio de Minkowski $\mathbb{M}_{2}$, entonces $\mathcal{S}^{\circ}=\mathcal{S}$ y $\mathcal{S}+\mathcal{S}^{[\perp]}=\mathcal{S} \neq \mathbb{M}_{2}$.

Sin embargo, todo subespacio cerrado de un espacio de Krein $\mathcal{H}$ admite un complemento cerrado, es decir, existe un subespacio cerrado $\mathcal{T}$ de $\mathcal{H}$ tal que

$$
\mathcal{H}=\mathcal{S}+\mathcal{T} \quad \text { y } \quad \mathcal{S} \cap \mathcal{T}=\{0\} .
$$

Esto puede verificarse eligiendo una descomposición canónica de $\mathcal{H}$ y tomando $\mathcal{T}=\mathcal{S}^{\perp}$, donde el ortogonal corresponde al producto interno del espacio de Hilbert asociado $|\mathcal{H}|$. Notemos que $\mathcal{S}$ y $\mathcal{T}$ son ortogonales respecto al producto interno de $|\mathcal{H}|$ pero no lo son respecto a la métrica indefinida de $\mathcal{H}$.

El resto de la subsección estará dedicado a identificar y caracterizar aquellos subespacios que tienen un complemento $J$-ortogonal, es decir, aquellos para los cuales

$$
\mathcal{H}=\mathcal{S} \oplus \mathcal{S}^{[\perp]} .
$$

Para ello introduciremos una nueva herramienta, el operador gramiano asociado a un subespacio cerrado de un espacio de Krein.

\section{El operador gramiano}

Sea $\mathcal{S}$ un subespacio cerrado de un espacio de Krein $\mathcal{H}=\mathcal{H}_{+} \oplus \mathcal{H}_{-}$con simetría canónica $J$. Consideremos la restricción de la métrica indefinida de $\mathcal{H}$ a $\mathcal{S}$ :

$$
[x, y]_{\mathcal{S}}=[x, y]_{\mathcal{H}} \quad \text { si } x, y \in \mathcal{S} .
$$

Luego, $[,]_{\mathcal{S}}$ es una forma sesquilineal acotada sobre el espacio de Hilbert $|\mathcal{S}|$ y, por consiguiente, existe un (único) operador autoadjunto $G_{\mathcal{S}} \in L(|\mathcal{S}|)$ tal que

$$
[x, y]_{\mathcal{S}}=\left\langle G_{\mathcal{S}} x, y\right\rangle_{|\mathcal{S}|} \quad \text { si } x, y \in \mathcal{S} .
$$

Si llamamos $P_{\mathcal{S}} \in L(|\mathcal{H}|,|\mathcal{S}|)$ a la proyección ortogonal con rango $\mathcal{S}$ tenemos que,

$$
\left\langle G_{\mathcal{S}} x, y\right\rangle_{|\mathcal{S}|}=[x, y]=\langle J x, y\rangle=\left\langle J x, P_{\mathcal{S}} y\right\rangle=\left\langle P_{\mathcal{S}} J x, y\right\rangle_{|\mathcal{S}|}
$$

para todo $x, y \in \mathcal{S}$, es decir, $G_{\mathcal{S}}=\left.P_{\mathcal{S}} J\right|_{\mathcal{S}}$.

Definición. El operador $G_{\mathcal{S}}: \mathcal{S} \rightarrow \mathcal{S}$ se denomina operador de Gram (o gramiano) de $\mathcal{S}$.

Dado un subespacio cerrado $\mathcal{S}$, notemos que $G_{\mathcal{S}} \in L(|\mathcal{S}|)$ es un operador autoadjunto cuyo núcleo y rango son fácilmente caracterizables:

$$
N\left(G_{\mathcal{S}}\right)=\mathcal{S}^{\circ}, \quad R\left(G_{\mathcal{S}}\right) \subseteq \mathcal{S} \ominus \mathcal{S}^{\circ}
$$

De hecho, dado $s \in \mathcal{S}$, notemos que $s \in N\left(G_{\mathcal{S}}\right)$ si y sólo si $P_{\mathcal{S}} J s=0$, o equivalentemente, $J s \in \mathcal{S}^{\perp}$. Es decir, $N\left(G_{\mathcal{S}}\right)=\mathcal{S} \cap \mathcal{S}^{[\perp]}$. Luego, $R\left(G_{\mathcal{S}}\right) \subseteq \overline{R\left(G_{\mathcal{S}}\right)}=N\left(G_{\mathcal{S}}\right)^{\perp}=\mathcal{S} \cap\left(\mathcal{S}^{\circ}\right)^{\perp}=\mathcal{S} \ominus \mathcal{S}^{\circ}$.

Como resultado de la caracterización del núcleo de $G_{\mathcal{S}}$, podemos afirmar que:

Proposición 1.2.28. Sea $\mathcal{S}$ un subespacio cerrado de un espacio de Krein $\mathcal{H}$. Luego, $\mathcal{S}$ es no degenerado si y sólo si $N\left(G_{\mathcal{S}}\right)=\{0\}$. 
Proposición 1.2.29. Si $\mathcal{S}$ es un subespacio cerrado de un espacio de Krein $\mathcal{H}$, entonces

$$
\mathcal{H}=\overline{\mathcal{S}+\mathcal{S}^{[\perp]}} \oplus J\left(\mathcal{S}^{\circ}\right)
$$

donde la suma directa es ortogonal respecto al producto interno de $|\mathcal{H}|$.

Demostración. Por las propiedades del subespacio $J$-ortogonal, tenemos que $\left(J \mathcal{S}^{\circ}\right)^{\perp}=\left(\mathcal{S}^{\circ}\right)^{[\perp]}=$ $\left(\mathcal{S} \cap \mathcal{S}^{[\perp]}\right)^{[\perp]}=\overline{\mathcal{S}+\mathcal{S}^{[\perp]}}$, y por lo tanto vale la Ec. (1.2.13).

Corolario 1.2.30. Sea $\mathcal{S}$ un subespacio cerrado de un espacio de Krein $\mathcal{H}$. Entonces, $\mathcal{H}=$ $\overline{\mathcal{S}+\mathcal{S}}{ }^{[\perp]}$ si y sólo si $\mathcal{S}$ es no degenerado.

Definición. Sea $\mathcal{H}$ un espacio de Krein, y un vector de $\mathcal{H}$ y $\mathcal{S}$ un subespacio cerrado de $\mathcal{H}$. Diremos que $x \in \mathcal{S}$ es una proyección $J$-ortogonal de $y \in \mathcal{H}$ en el subespacio $\mathcal{S}$ si $y-x[\perp] \mathcal{S}$.

Es importante destacar que si $\mathcal{S}$ es un subespacio degenerado, para un vector fijo $y \in \mathcal{H}$, pueden existir más de una proyección $J$-ortogonal de $y$. De hecho, si $x \in \mathcal{S}$ es una de ellas y $n \in \mathcal{S}^{\circ}$ entonces $x+n$ también es una proyección $J$-ortogonal de $y$. Por lo tanto, si $x \in \mathcal{S}$ es una proyección $J$-ortogonal de $y$, el conjunto de proyecciones $J$-ortogonales de $y$ coincide con la variedad afín

$$
x+\mathcal{S}^{\circ}
$$

Lema 1.2.31. Si existe un $y_{0} \in \mathcal{H}$ con una única proyección $J$-ortogonal entonces $\mathcal{S}$ es no degenerado. Además, si $\mathcal{S}$ es un subespacio no degenerado, para cada $y \in \mathcal{H}$ existe a lo sumo una proyección $J$-ortogonal sobre $\mathcal{S}$.

Demostración. Anteriormente mencionamos que, si $x_{0} \in \mathcal{S}$ es una proyección $J$-ortogonal de $y_{0} \in \mathcal{H}$, entonces $x+\mathcal{S}^{\circ}$ es el conjunto de proyecciones $J$-ortogonales de $y$. Luego, si $y_{0}$ admite una única proyección $J$-ortogonal, resulta que $\mathcal{S}^{\circ}=\{0\}$. Por lo tanto, $\mathcal{S}$ es no degenerado.

Además, suponiendo que $\mathcal{S}$ es no degenerado, si para algún vector $y \in \mathcal{H}$ existen dos proyecciones $J$-ortogonales $x_{1}$ y $x_{2}$ sobre $\mathcal{S}\left(x_{1} \neq x_{2}\right)$, luego el vector $z=x_{1}-x_{2}$ es isotrópico a $\mathcal{S}$, lo que resulta absurdo.

Finalmente, caracterizaremos la existencia de una proyección $J$-ortogonal - para un vector fijo $y \in \mathcal{H}$ - en términos de la existencia de solución para una ecuación de la forma $G_{\mathcal{S}} x=z$, siendo $z \in \mathcal{S}$ un vector que depende de $y$.

Lema 1.2.32. Dado un subespacio cerrado $\mathcal{S}$ de un espacio de Krein $\mathcal{H}$ con simetría fundamental $J$, sean $P_{\mathcal{S}}$ la proyección ortogonal (respecto al producto interno de $|\mathcal{H}|$ ) sobre $\mathcal{S}$ y sea $G_{\mathcal{S}}=$ $\left.P_{\mathcal{S}} J\right|_{\mathcal{S}}$ el operador de Gram. Dado y $\in \mathcal{H}$, y tiene una proyección $J$-ortogonal sobre $\mathcal{S}$ si y sólo si existe un vector $x \in \mathcal{S}$ tal que

$$
G_{\mathcal{S}} x=P_{\mathcal{S}} J y
$$

Demostración. La existencia de una proyección ortogonal $x \in \mathcal{S}$ es equivalente a que, para todo $s \in \mathcal{S},[y-x, s]=0$. Luego, $\left\langle P_{\mathcal{S}} J y-G_{\mathcal{S}} x, s\right\rangle=\langle J(y-x), s\rangle=[y-x, s]=0$ para todo $s \in \mathcal{S}$, es decir, $P_{\mathcal{S}} J y=G_{\mathcal{S}} x$. Como todo el argumento es reversible, el lema queda probado.

El próximo paso es caracterizar aquellos subespacios $\mathcal{S}$ de un espacio de Krein $\mathcal{H}$ para los cuales todo $y \in \mathcal{H}$ admite una proyección $J$-ortogonal. Notemos que, en este caso

$$
\mathcal{H}=\mathcal{S} \dot{+} \mathcal{S}^{[\perp]} .
$$


Definición. Un subespacio $\mathcal{S}$ se dice proyectivamente completo si

$$
\mathcal{H}=\mathcal{S}+\mathcal{S}^{[\perp]}
$$

Notemos que, por el Corolario 1.2.30, todo subespacio proyectivamente completo es no degenerado. Por lo tanto, si $\mathcal{S}$ es un subespacio proyectivamente completo de un espacio de Krein $\mathcal{H}$ entonces

$$
\mathcal{H}=\mathcal{S}+\mathcal{S}^{[\perp]} .
$$

Proposición 1.2.33. Si $\mathcal{S}$ es un subespacio proyectivamente completo de $\mathcal{H}$ entonces $\mathcal{S}$ es cerrado. Además, $\mathcal{S}$ es proyectivamente completo si y sólo si $\mathcal{S}^{[\perp]}$ lo es.

Demostración. Supongamos que $\mathcal{S}$ es proyectivamente completo y sea $x \in \overline{\mathcal{S}}$. Descompongamos a $x$ como $x=y+z$ con $y \in \mathcal{S}, z \in \mathcal{S}^{[\perp]}$. Luego, $z=x-y \in \overline{\mathcal{S}} \cap \mathcal{S}^{[\perp]}=\mathcal{S}^{[\perp][\perp]} \cap \mathcal{S}^{[\perp]}=$ $\left(\mathcal{S}+\mathcal{S}^{[\perp]}\right)^{[\perp]}=\{0\}$ y entonces $x \in \mathcal{S}$. Por lo tanto, $\mathcal{S}$ es un subespacio cerrado de $\mathcal{H}$. La segunda afirmación en la proposición es una consecuencia directa de la igualdad $\mathcal{S}^{[\perp][\perp]}=\overline{\mathcal{S}}=\mathcal{S}$.

Teorema 1.2.34. Sea $\mathcal{S}$ un subespacio cerrado de un espacio de Krein $\mathcal{H}$. Entonces, $\mathcal{S}$ es proyectivamente completo si y sólo si $G_{\mathcal{S}}$ es inversible.

Demostración. Supongamos que $\mathcal{S}$ es proyectivamente completo. Como $\mathcal{S}$ es no degenerado, $N\left(G_{\mathcal{S}}\right)=\{0\}$, es decir, $G_{\mathcal{S}}$ es inyectivo. Además, por la Proposición 1.1.18, $\mathcal{H}=\mathcal{S}+\mathcal{S}^{[\perp]}$ implica que $\mathcal{S}^{\perp}+J(\mathcal{S})$ es cerrado, y luego - aplicando otra vez la Proposición 1.1.18 - vemos que $P_{\mathcal{S}} J(\mathcal{S})=R\left(G_{\mathcal{S}}\right)$ también es cerrado. Por lo tanto, $G_{\mathcal{S}}$ es inversible.

La otra implicación se demuestra invirtiendo los argumentos anteriores.

Corolario 1.2.35. Sea $\mathcal{F}$ un subespacio de dimensión finita de un espacio de Krein $\mathcal{H}$. Entonces, $\mathcal{F}$ es proyectivamente completo si y sólo si $\mathcal{F}$ es no degenerado.

Demostración. Dado que un operador actuando entre espacios vectoriales de dimensión finita es biyectivo si y sólo si es inyectivo, el corolario se desprende de la caracterización de $N\left(G_{\mathcal{F}}\right)$ y el teorema anterior.

Ejemplo 1.2.36. Sean $e_{1}, \ldots, e_{n}$ vectores linealmente independientes en un espacio de Krein $\mathcal{H}$ y sea $\mathcal{S}=\operatorname{span}\left\{e_{1}, \ldots, e_{n}\right\}$. Luego, $\mathcal{S}$ es un subespacio proyectivamente completo de $\mathcal{H}$ si y sólo si la matriz

$$
G=G\left(e_{1}, \ldots, e_{n}\right)=\left(\left[e_{j}, e_{i}\right]_{\mathcal{H}}\right)_{i, j=1, \ldots, n}
$$

es inyectiva.

De hecho, $G$ es la representación matricial del operador gramiano $G_{\mathcal{S}}$ respecto a la base (algebraica) $e_{1}, \ldots, e_{n}$ de $\mathcal{S}$.

\section{Subespacios de Krein}

Definición. Un subespacio de Krein de un espacio de Krein $\mathcal{H}$ es un subespacio cerrado $\mathcal{S}$ que es en sí mismo un espacio de Krein respecto a la métrica de $\mathcal{K}$, es decir, $\left(\mathcal{S},[,]_{\mathcal{S}}\right)$ es un espacio de Krein con la métrica

$$
[x, y]_{\mathcal{S}}=[x, y]_{\mathcal{H}}, \quad x, y \in \mathcal{S} .
$$

Definición. Sea $\mathcal{H}$ un espacio de Krein. Un operador $Q \in L(\mathcal{H})$ es una proyección J-ortogonal si $Q^{2}=Q$ y $Q^{\#}=Q$. 
El siguiente resultado muestra que las proyecciones $J$-ortogonales de un espacio de Krein son similares a las proyecciones ortogonales de un espacio de Hilbert. Sin embargo, no todo subespacio cerrado de un espacio de Krein es el rango de una proyección $J$-ortogonal.

Teorema 1.2.37. Si $\mathcal{S}$ es un subespacio cerrado de un espacio de Krein $\mathcal{H}$, las siguientes condiciones son equivalentes:

1. $\mathcal{S}$ es un subespacio de Krein;

2. $\mathcal{H}=\mathcal{S}+\mathcal{S}^{[\perp]}$;

3. $\mathcal{S}$ es el rango de una proyección J-ortogonal $Q$.

Demostración. 3. $\Rightarrow 1$ : Sea $\mathcal{S}=R(Q)$ para la proyección $J$-ortogonal $Q$. Usemos el Teorema 1.2.21 para factorizar a $Q$, i.e. $Q=D D^{\#}$ con $D \in L(\mathcal{D}, \mathcal{H})$ inyectivo. Como $Q^{2}=Q$,

$$
D D^{\#} D D^{\#}=D D^{\#} \text {, }
$$

y como $D$ es inyectivo, $D^{\#}$ tiene rango denso y tenemos que $D D^{\#} D=D$ y $D^{\#} D=I_{\mathcal{H}}$. Además, $R(D)=R\left(D D^{\#}\right)=\mathcal{S}$. Luego, hemos probado que $\mathcal{S}$ es un subespacio de Krein pues $D$ es un isomorfismo del espacio de Krein $\mathcal{D}$ sobre $\mathcal{S}$ en la métrica indefinida de $\mathcal{H}$.

2. $\Rightarrow$ 3.: Si $\mathcal{H}=\mathcal{S} \dot{+} \mathcal{S}^{[\perp]}$ entonces todo $z \in \mathcal{H}$ tiene una única representación de la forma

$$
z=x+y ; \quad \operatorname{con} x \in \mathcal{S}, y \in \mathcal{S}^{[\perp]} .
$$

La relación $Q z=x$ define un operador lineal que, por el Teorema del Gráfico Cerrado, resulta acotado. De la Ec. (1.2.15) deducimos que $Q$ es una proyección y claramente $\mathcal{S}=R(Q)$. Finalmente, dados dos vectores arbitrarios $z_{1}, z_{2} \in \mathcal{H}$, si para $i=1,2$ consideramos la descomposición $z_{i}=x_{i}+y_{i} \operatorname{con} x_{i} \in \mathcal{S}$ e $y_{i} \in \mathcal{S}^{[\perp]}$,

$$
\left[Q z_{1}, z_{2}\right]=\left[x_{1}, x_{2}+y_{2}\right]=\left[x_{1}, x_{2}\right]=\left[x_{1}+y_{1}, x_{2}\right]=\left[z_{1}, Q z_{2}\right] .
$$

Por lo tanto, $Q$ es una proyección $J$-ortogonal con rango $\mathcal{S}$.

1. $\Rightarrow$ 2. Supongamos que $\mathcal{S}$ es un espacio de Krein con la métrica indefinida de $\mathcal{H}$. Entonces $\mathcal{S}=\mathcal{S}_{+} \oplus \mathcal{S}_{-}$, donde $\mathcal{S}_{+}$es un espacio de Hilbert y $\mathcal{S}_{-}$es el antiespacio de un espacio de Hilbert (en ambos casos considerando la métrica indefinida de $\mathcal{H}$ ). Por el item 5. del Teorema 1.2.27, $\mathcal{S}_{ \pm} \subseteq \mathcal{H}_{ \pm}$para alguna descomposición canónica $\mathcal{H}=\mathcal{H}_{+} \oplus \mathcal{H}_{-}$de $\mathcal{H}$.

Como $\mathcal{S}_{+}$es un subespacio cerrado del espacio de Hilbert $\mathcal{H}_{+}$(dotado con la métrica indefinida), $\mathcal{H}_{+}=\mathcal{S}_{+} \oplus\left(\mathcal{H}_{+} \ominus \mathcal{S}_{+}\right)$y la suma es ortogonal (con respecto a la métrica indefinida). Análogamente, $\mathcal{H}_{-}=\mathcal{S}_{-} \oplus\left(\mathcal{H}_{-} \ominus \mathcal{S}_{-}\right)$. Además,

$$
\begin{aligned}
\mathcal{S}^{[\perp]} & =\left(\mathcal{S}_{+}+\mathcal{S}_{-}\right)^{[\perp]}=\mathcal{S}_{+}^{[\perp]} \cap \mathcal{S}_{-}^{[\perp]}= \\
& =\left(\left(\mathcal{H}_{+} \ominus \mathcal{S}_{+}\right) \oplus \mathcal{H}_{-}\right) \cap\left(\mathcal{H}_{+} \oplus\left(\mathcal{H}_{-} \ominus \mathcal{S}_{-}\right)\right)=\left(\mathcal{H}_{+} \ominus \mathcal{S}_{+}\right) \oplus\left(\mathcal{H}_{-} \ominus \mathcal{S}_{-}\right),
\end{aligned}
$$

es decir, $\mathcal{S}^{[\perp]}$ es la suma directa de $\mathcal{H}_{-} \ominus \mathcal{S}_{-}$y $\mathcal{H}_{+} \ominus \mathcal{S}_{+}$. Por lo tanto, $\mathcal{H}=\mathcal{S} \oplus \mathcal{S}^{[\perp]}$.

Corolario 1.2.38. Sea $\mathcal{S}$ un subespacio definido de un espacio de Krein $\mathcal{H}$. Entonces, $\mathcal{S}$ es uniformemente definido si y sólo si es proyectivamente completo.

Demostración. Es consecuencia de la Observación 1.2.26 y el teorema anterior. 


\section{Capítulo 2}

\section{Proyecciones autoadjuntas respecto a una métrica indefinida}

Fijado un espacio de Hilbert $\mathcal{H}$ con producto interno $\langle$,$\rangle , hay ocasiones en las que resulta$ útil perturbar el producto interno con un "peso", es decir, dado un operador positivo $A \in L(\mathcal{H})$ considerar la aplicación $\langle,\rangle_{A}: \mathcal{H} \times \mathcal{H} \rightarrow \mathbb{C}$ definida por

$$
\langle x, y\rangle_{A}=\langle A x, y\rangle, \quad x, y \in \mathcal{H} .
$$

Si el operador $A$ es inversible, es fácil probar que $\langle,\rangle_{A}$ también es un producto interno sobre $\mathcal{H}$ y además induce una norma en $\mathcal{H}$ equivalente a la inducida por el producto inicial $\langle$,$\rangle en el$ siguiente sentido: existen constantes $\alpha, \beta>0$ tales que

$$
\alpha\|x\|^{2} \leq\|x\|_{A}^{2} \leq \beta\|x\|^{2} \quad \text { para todo } x \in \mathcal{H},
$$

siendo $\|x\|^{2}=\langle x, x\rangle \mathrm{y}\|x\|_{A}^{2}=\langle x, x\rangle_{A}$.

En cambio, si $A$ no es inversible, la forma sesquilineal no negativa $\langle,\rangle_{A}$ resulta degenerada. Por ejemplo, si el núcleo de $A$ es no trivial, $\langle y, y\rangle_{A}=0$ para todo $y \in N(A)$. En varios trabajos [19, 20, 21, 22, 23, 24] G. Corach, A. Maestripieri y D. Stojanoff estudiaron las proyecciones autoadjuntas con respecto a estas formas - a las que denominaron proyecciones $A$-autoadjuntas - y sus aplicaciones al estudio de complementos de Schur, problemas de cuadrados mínimos, splines abstractos, etc.

Dado un operador autoadjunto $B \in L(\mathcal{H})$, la aplicación $\langle,\rangle_{B}: \mathcal{H} \times \mathcal{H} \rightarrow \mathbb{C}$ definida por

$$
\langle x, y\rangle_{B}=\langle B x, y\rangle, \quad x, y \in \mathcal{H},
$$

no es un producto interno sino una métrica indefinida sobre $\mathcal{H}$ (ver la sección 1.2 de los Preliminares). Como continuación a los trabajos anteriormente mencionados, nos proponemos estudiar bajo qué condiciones existen y qué características tienen las proyecciones autoadjuntas con respecto a esta métrica indefinida.

\subsection{Definiciones y propiedades básicas}

Sea $\mathcal{H}$ un espacio de Hilbert con producto interno $\langle$,$\rangle y consideremos la métrica indefinida$ sobre $\mathcal{H}$ inducida por un operador autoadjunto $B \in L(\mathcal{H})$. 
Definición. Fijado $B \in L(\mathcal{H})^{s}$, un operador $T \in L(\mathcal{H})$ es $B$-autoadjunto si

$$
\langle T x, y\rangle_{B}=\langle x, T y\rangle_{B} \quad \text { para todo } x, y \in \mathcal{H} .
$$

Notemos que $T$ es $B$-autoadjunto si y sólo si cumple la identidad $B T=T^{*} B$.

El objetivo de este capítulo es estudiar la existencia y unicidad de proyecciones $B$-autoadjuntas. En particular, fijado un subespacio cerrado $\mathcal{S}$ de $\mathcal{H}$, nos interesaran aquellas proyecciones $B$-autoadjuntas con rango $\mathcal{S}$.

Definición. Sean $\mathcal{S}$ un subespacio cerrado de $\mathcal{H}$ y $B \in L(\mathcal{H})^{s}$. El par $(B, \mathcal{S})$ es compatible si existe una proyección $B$-autoadjunta con rango $\mathcal{S}$, i.e. si el conjunto

$$
\mathcal{P}(B, \mathcal{S}):=\left\{Q \in \mathcal{Q}: R(Q)=\mathcal{S}, B Q=Q^{*} B\right\}
$$

no es vacío.

Si $\mathcal{S}$ es un subespacio de $\mathcal{H}$ y $B \in L(\mathcal{H})^{s}$, el subespacio $B$-ortogonal a $\mathcal{S}$ está dado por

$$
\mathcal{S}^{\perp_{B}}:=\{x \in \mathcal{H}:\langle B x, s\rangle=0 \text { para todo } s \in \mathcal{S}\} .
$$

Observemos que, como $B$ es autoadjunto, $\mathcal{S}^{\perp_{B}}=B^{-1}\left(\mathcal{S}^{\perp}\right)=B(\mathcal{S})^{\perp}$. Las relaciones básicas que cumple $\perp_{B}$ respecto a la suma o a la intersección de subespacios ya las hemos mencionado en la Sección 1.2 de los Preliminares.

El siguiente lema puede encontrarse, por ejemplo, en [38, página 404] ó en [19, Lema 3.2].

Lema 2.1.1 (Krein). Sea $Q \in L(\mathcal{H})$ una proyección con $R(Q)=\mathcal{S}$. Entonces, $Q \in \mathcal{P}(B, \mathcal{S})$ si y sólo si $N(Q) \subseteq B^{-1}\left(\mathcal{S}^{\perp}\right)$.

Demostración. Si $Q$ es $B$-autoadjunta y $x \in N(Q)$ entonces $Q^{*} B x=B Q x=0$, es decir, $B x \in N\left(Q^{*}\right)=R(Q)^{\perp}=\mathcal{S}^{\perp}$. Por lo tanto, $x \in B^{-1}\left(\mathcal{S}^{\perp}\right)$. Recíprocamente, si $N(Q) \subseteq B^{-1}\left(\mathcal{S}^{\perp}\right)$ y $x \in \mathcal{H}$,

$$
Q^{*} B x=Q^{*} B Q x+Q^{*} B(I-Q) x=Q^{*} B Q x
$$

porque $B(I-Q) x \in \mathcal{S}^{\perp}=R(Q)^{\perp}=N\left(Q^{*}\right)$. Entonces, $Q^{*} B=Q^{*} B Q$ y adjuntando obtenemos que $B Q=Q^{*} B Q=Q^{*} B$.

Como consecuencia del lema anterior tenemos que $(B, \mathcal{S})$ es compatible si y sólo si

$$
\mathcal{H}=\mathcal{S}+B^{-1}\left(\mathcal{S}^{\perp}\right) .
$$

Anteriormente mencionamos que si $A \in G L(\mathcal{H})^{+}$entonces $(A, \mathcal{S})$ es compatible para todo subespacio cerrado $\mathcal{S}$ de $\mathcal{H}$, ya que $\langle,\rangle_{A}$ es un producto interno sobre $\mathcal{H}$. Por otra parte, G. Corach et al. mostraron que, si $A \in L(\mathcal{H})^{+}$y $\mathcal{S}$ es un subespacio de dimensión finita, entonces $\mathcal{P}(A, \mathcal{S}) \neq \varnothing($ ver $[19$, Teorema 6.2]).

Sin embargo, el siguiente ejemplo muestra que existen pares $(B, \mathcal{S})$ con $B \in G L(\mathcal{H})^{s}$ tales que $\mathcal{P}(B, \mathcal{S})=\varnothing$, aún cuando el espacio de Hilbert $\mathcal{H}$ es de dimensión finita.

Ejemplo 2.1.2. Sea $\mathcal{H}=\mathbb{C}^{2}$, consideremos el subespacio $\mathcal{S}=\left\{(x, y) \in \mathbb{C}^{2}: y=-x\right\}$ y el operador

$$
J=\left(\begin{array}{cc}
1 & 0 \\
0 & -1
\end{array}\right) \in G L\left(\mathbb{C}^{2}\right)^{s} .
$$

Observemos que $J^{-1}\left(\mathcal{S}^{\perp}\right)=\mathcal{S}$ y entonces $\mathcal{S}+J^{-1}\left(\mathcal{S}^{\perp}\right)=\mathcal{S} \neq \mathbb{C}^{2}$. Luego, $(J, \mathcal{S})$ no es compatible. 
Definición. Dado $B \in L(\mathcal{H})^{s}$ y un subespacio cerrado $\mathcal{S}$ de $\mathcal{H}$, se define la parte B-isotrópica de $\mathcal{S}$ como el subespacio

$$
\mathcal{N}:=\mathcal{S} \cap B(\mathcal{S})^{\perp}
$$

En el caso en que $A \in L(\mathcal{H})^{+}$y $\mathcal{S}$ es un subespacio cerrado de $\mathcal{H}$, es fácil probar que $\mathcal{N}=\mathcal{S} \cap N(A)$. De hecho, si $x \in \mathcal{N},\left\|A^{1 / 2} x\right\|^{2}=\langle A x, x\rangle=0$, y entonces $x \in N\left(A^{1 / 2}\right)=N(A)$. Por otra parte, $N(A)=A^{-1}(\{0\}) \subseteq A^{-1}\left(\mathcal{S}^{\perp}\right)=A(\mathcal{S})^{\perp}$ implica que $\mathcal{S} \cap N(A) \subseteq \mathcal{N}$.

En general, esta identidad no vale para operadores autoadjuntos. Por ejemplo, para el par $(J, \mathcal{S})$ del Ejemplo 2.1.2, la parte $B$-isotrópica de $\mathcal{S}$ coincide con $\mathcal{S}$ mientras que $\mathcal{S} \cap N(J)=\{0\}$. Sin embargo,

Lema 2.1.3. Sean $B \in L(\mathcal{H})^{s}$ y $\mathcal{S}$ un subespacio cerrado de $\mathcal{H}$ tales que $(B, \mathcal{S})$ es compatible. Entonces, $\mathcal{N}=\mathcal{S} \cap N(B)$.

Demostración. La inclusión $\mathcal{S} \cap N(B) \subseteq \mathcal{N}$ vale en cualquier caso, ya que $N(B)=B^{-1}(\{0\}) \subseteq$ $B^{-1}\left(\mathcal{S}^{\perp}\right)=B(\mathcal{S})^{\perp}$. Ahora, supongamos que $(B, \mathcal{S})$ es compatible y consideremos $Q \in \mathcal{P}(B, \mathcal{S})$. Si $x \in \mathcal{N}$ e $y \in \mathcal{H}$ entonces,

$$
\langle B x, y\rangle=\langle B Q x, y\rangle=\left\langle Q^{*} B x, y\right\rangle=\langle B x, Q y\rangle=0
$$

porque $B x \in \mathcal{S}^{\perp}$. Por lo tanto, $B x=0$ i.e. $x \in \mathcal{S} \cap N(B)$.

Proposición 2.1.4. Sean $\mathcal{S}$ un subespacio cerrado de $\mathcal{H}$ y $B \in L(\mathcal{H})^{s}$. Entonces, $(B, \mathcal{S})$ es compatible si y sólo si $\mathcal{N}=\mathcal{S} \cap N(B)$ y $(B, \mathcal{S} \ominus \mathcal{N})$ es compatible.

Demostración. $\operatorname{Si}(B, \mathcal{S})$ es compatible, por el Lema 2.1.3, sabemos que $\mathcal{N}=\mathcal{S} \cap N(B)$. Además, $\mathcal{H}=\mathcal{S}+B(\mathcal{S})^{\perp}=\mathcal{S} \ominus \mathcal{N}+B(\mathcal{S})^{\perp} \subseteq \mathcal{S} \ominus \mathcal{N}+B(\mathcal{S} \ominus \mathcal{N})^{\perp}$, es $\operatorname{decir}(B, \mathcal{S} \ominus \mathcal{N})$ es compatible.

Recíprocamente, si $\mathcal{N}=\mathcal{S} \cap N(B)$ es claro que $B(\mathcal{S} \ominus \mathcal{N})=B(\mathcal{S})$. Luego, si $(B, \mathcal{S} \ominus \mathcal{N})$ es compatible, tenemos que $\mathcal{H}=\mathcal{S} \ominus \mathcal{N}+B(\mathcal{S} \ominus \mathcal{N})^{\perp}=\mathcal{S} \ominus \mathcal{N} \dot{+} B(\mathcal{S})^{\perp}=\mathcal{S}+B(\mathcal{S})^{\perp}$. Por lo tanto, $(B, \mathcal{S})$ es compatible.

Si consideramos un operador $A \in L(\mathcal{H})^{+}$, hemos mencionado que $\mathcal{N}=\mathcal{S} \cap N(A)$ y, por la proposición anterior, $(A, \mathcal{S})$ es compatible si y sólo si $(A, \mathcal{S} \ominus \mathcal{N})$ es compatible. En cambio, si $B \in L(\mathcal{H})^{s}$, la compatibilidad de $(B, \mathcal{S} \ominus \mathcal{N})$ no es suficiente para garantizar la compatibilidad de $(B, \mathcal{S})$. Veamos un ejemplo:

Ejemplo 2.1.5. Sea $\left\{e_{1}, e_{2}, e_{3}, e_{4}\right\}$ la base canónica de $\mathbb{R}^{4}, \mathcal{S}=\operatorname{span}\left\{e_{1}, e_{2}, e_{3}\right\}$ - el subespacio generado por $\left\{e_{1}, e_{2}, e_{3}\right\}-\mathrm{y}$

$$
B=\left(\begin{array}{llll}
1 & 0 & 0 & 1 \\
0 & 1 & 0 & 0 \\
0 & 0 & 0 & 1 \\
1 & 0 & 1 & 1
\end{array}\right) \in G L\left(\mathbb{R}^{4}\right)^{s}
$$

Luego, $\mathcal{N}=\operatorname{span}\left\{e_{3}\right\}, \mathcal{S} \ominus \mathcal{N}=\operatorname{span}\left\{e_{1}, e_{2}\right\}$ y el par $(B, \mathcal{S} \ominus \mathcal{N})$ es compatible. Pero $(B, \mathcal{S})$ no es compatible ya que $B(\mathcal{S})=\operatorname{span}\left\{e_{1}, e_{2}, e_{4}\right\}$ y luego $\mathcal{S}+B(\mathcal{S})^{\perp}=\mathcal{S} \neq \mathcal{H}$. 


\subsubsection{El operador de Gram inducido por un operador autoadjunto}

Definición. Dados $B \in L(\mathcal{H})^{s}$ y un subespacio cerrado $\mathcal{S}$ de $\mathcal{H}$, el operador de Gram de $\mathcal{S}$ inducido por $B$ (o $B$-gramiano) está definido por

$$
G_{B, \mathcal{S}}:=P B P
$$

siendo $P$ la proyección ortogonal sobre $\mathcal{S}$.

Dado $x \in \mathcal{H}$, notemos que $x \in N\left(G_{B, \mathcal{S}}\right)$ si y sólo si $B P x \in \mathcal{S}^{\perp}$, o equivalentemente, si $P x \in B^{-1}\left(\mathcal{S}^{\perp}\right)=B(\mathcal{S})^{\perp}$. Vemos entonces que

$$
N\left(G_{B, \mathcal{S}}\right)=\mathcal{S}^{\perp}+\mathcal{N} \quad \text { y } \overline{R\left(G_{B, \mathcal{S}}\right)}=N\left(G_{B, \mathcal{S}}\right)^{\perp}=\mathcal{S} \ominus \mathcal{N}
$$

Observación 2.1.6. Si $J \in L(\mathcal{H})$ es una simetría - i.e. $J=J^{*}=J^{-1}$ - y $\mathcal{S}$ es un subespacio cerrado de $\mathcal{H}$, el $J$-gramiano $G_{J, \mathcal{S}}$ está relacionado con el operador de Gram $G_{\mathcal{S}}$ dado por la Ec. (1.2.12) (definido en términos del espacio de Krein que resulta de dotar a $\mathcal{H}$ con la métrica indefinida $\left.\langle,\rangle_{J}\right)$. De hecho,

$$
G_{\mathcal{S}}=\left.G_{J, \mathcal{S}}\right|_{\mathcal{S}}
$$

Luego, el siguiente resultado puede considerarse una generalización del Teorema 1.2.34.

Proposición 2.1.7. Dados $B \in L(\mathcal{H})^{s}$ y un subespacio cerrado $\mathcal{S}$ de $\mathcal{H}$, son equivalentes:

1. $(B, \mathcal{S})$ es compatible;

2. $R(P B) \subseteq R(P B P)$;

3. la ecuación $G_{B, \mathcal{S}} X=P B$ admite una solución acotada.

Demostración. 1. $\Rightarrow$ 2.: $\mathrm{Si}(B, \mathcal{S})$ es compatible entonces

$$
\mathcal{H}=\mathcal{S}+B^{-1}\left(\mathcal{S}^{\perp}\right)=\mathcal{S}+B^{-1}(N(P))=\mathcal{S}+N(P B) .
$$

Pro lo tanto, $R(P B) \subseteq P B(\mathcal{S})=R(P B P)$.

2. $\Rightarrow$ 3.: Es consecuencia del Teorema de Douglas.

3. $\Rightarrow$ 1.: Supongamos que la ecuación $(P B P) X=P B$ admite una solución en $L(\mathcal{H})$. Si $D \in$ $L(\mathcal{H})$ es la solución reducida de esta ecuación, tenemos que $N(D)=N(P B)=B^{-1}(N(P))=$ $B(\mathcal{S})^{\perp}$ y $R(D) \subseteq \overline{R(P B P)}=\mathcal{S} \ominus \mathcal{N}$. Además, por el Corolario 1.1.3, $D \in \mathcal{Q}$. Luego,

$$
\mathcal{H}=R(D)+N(D) \subseteq \mathcal{S} \ominus \mathcal{N}+B(\mathcal{S})^{\perp}=\mathcal{S}+B(\mathcal{S})^{\perp},
$$

i.e. el $\operatorname{par}(B, \mathcal{S})$ es compatible.

Lema 2.1.8. Sea $(B, \mathcal{S})$ un par compatible y consideremos $\mathcal{T}=\mathcal{S} \ominus \mathcal{N}$. Luego,

1. $G_{B, \mathcal{S}}=G_{B, \mathcal{T}}$.

2. $\mathcal{T} \cap B(\mathcal{T})^{\perp}=\{0\}$ y la restricción del operador de Gram $G_{B, \mathcal{T}}$ a $\mathcal{T}$,

$$
G=\left.P_{\mathcal{T}} B\right|_{\mathcal{T}}: \mathcal{T} \rightarrow \mathcal{T} \quad \text { es inyectivo }
$$


Demostración. Por la Proposición 2.1.3 sabemos que $\mathcal{N} \subseteq N(B)$. Entonces $B(\mathcal{S})=B(\mathcal{T})$, o equivalentemente, $B P=B P_{\mathcal{T}}$. Como $B P_{\mathcal{N}}=P_{\mathcal{N}} B=0$, vemos que

$$
G_{B, \mathcal{S}}=P B P=P B\left(P_{\mathcal{T}}+P_{\mathcal{N}}\right)=\left(P_{\mathcal{T}}+P_{\mathcal{N}}\right) B P_{\mathcal{T}}=P_{\mathcal{T}} B P_{\mathcal{T}}=G_{B, \mathcal{T}}
$$

Notemos que la parte $B$-isotrópica de $\mathcal{T}$ es trivial; de hecho, como $B(\mathcal{T})=B(\mathcal{S})$, tenemos que $\mathcal{T} \cap B(\mathcal{T})^{\perp}=\mathcal{T} \cap B(\mathcal{S})^{\perp}=\mathcal{S} \cap \mathcal{N}^{\perp} \cap B(\mathcal{S})^{\perp}=\mathcal{N} \cap \mathcal{N}^{\perp}=\{0\}$. Además, dado $x \in \mathcal{S} \ominus \mathcal{N}$, $x \in N(G)$ si y sólo si $B x \in \mathcal{T}^{\perp}$, o equivalentemente, $x \in \mathcal{T} \cap B(\mathcal{T})^{\perp}=\{0\}$. Por lo tanto, $G$ es inyectivo.

Definición. Dado un operador $B \in L(\mathcal{H})^{s}$, un vector $x \in \mathcal{H}$ se dice $B$-positivo si es positivo respecto a la métrica indefinida $\langle,\rangle_{B}$, es decir, $\langle x, x\rangle_{B}>0$. Por su parte, diremos que un subespacio $\mathcal{S}$ de $\mathcal{H}$ es $B$-positivo si todo vector no nulo $x \in \mathcal{S}$ es $B$-positivo. De la misma manera, definimos vectores (y subespacios) $B$-no negativos, $B$-neutros, $B$-negativos y $B$-no positivos.

Definición. Dado un operador $B \in L(\mathcal{H})^{s}$, un subespacio $\mathcal{S}$ de $\mathcal{H}$ es uniformemente B-positivo si existe una constante $\alpha>0$ tal que, para todo vector no nulo $x \in \mathcal{S}$,

$$
\langle x, x\rangle_{B} \geq \alpha\|x\|^{2} .
$$

Análogamente, diremos que $\mathcal{S}$ es uniformemente $B$-negativo si existe una constante $\alpha>0$ tal que, para todo vector no nulo $x \in \mathcal{S},\langle x, x\rangle_{B} \leq-\alpha\|x\|^{2}$.

Si $\mathcal{S}$ es un subespacio $J$-positivo de un espacio de Krein $\mathcal{H}$ con simetría fundamental $J$, por el Corolario 1.2.38 sabemos que existe una proyección $J$-autoadjunta sobre $\mathcal{S}$ si y sólo si $\mathcal{S}$ es uniformemente $J$-positivo i.e. existe un $\alpha>0$ tal que $[x, x] \geq \alpha\|x\|^{2}$ para todo $x \in \mathcal{S}$.

Si $B \in G L(\mathcal{H})^{s}$ y $\mathcal{S}$ es un subespacio $B$-positivo de $\mathcal{H}$ entonces es fácil ver que $(B, \mathcal{S})$ es compatible si y sólo si $\mathcal{S}$ es uniformemente $B$-positivo (ver la Proposición 2.1.11). Sin embargo, sólo una de estas implicaciones es cierta para un operador autoadjunto arbitrario. Enunciaremos los siguientes resultados para subespacios $B$-positivos, pero pueden deducirse fácilmente los análogos para subespacios $B$-negativos.

Proposición 2.1.9. Si $\mathcal{S}$ es uniformemente B-positivo entonces $(B, \mathcal{S})$ es compatible.

Demostración. Supongamos que $\mathcal{S}$ es uniformemente $B$-positivo. Luego, para todo $x \in \mathcal{H}$,

$$
\left\langle G_{B, \mathcal{S}} x, x\right\rangle=\left\langle P_{\mathcal{S}} x, P_{\mathcal{S}} x\right\rangle_{B} \geq \alpha\left\|P_{\mathcal{S}} x\right\|^{2} .
$$

Entonces, $G_{B, \mathcal{S}}$ es acotado inferiormente (en $N\left(G_{B, \mathcal{S}}\right)^{\perp}$ ) y, en consecuencia, $R\left(G_{B, \mathcal{S}}\right)$ es cerrado. Además, $\mathcal{N}=\{0\}$ ya que, si $x \in \mathcal{N}$, entonces $0=\langle B x, x\rangle=\left\langle G_{B, \mathcal{S}} x, x\right\rangle \geq \alpha\|x\|^{2}$. Por lo tanto, $R\left(G_{B, \mathcal{S}}\right)=\mathcal{S}$. Luego, por el Teorema de Douglas, la ecuación $G_{B, \mathcal{S}} X=P B$ tiene una solución acotada y, aplicando la Proposición 2.1.7, vemos que $(B, \mathcal{S})$ es compatible.

Observación 2.1.10. Dado un subespacio (cerrado) $B$-positivo $\mathcal{S}$ de $\mathcal{H}$, la compatibilidad de $(B, \mathcal{S})$ no es una condición suficiente para asegurar que $\mathcal{S}$ sea uniformemente $B$-positivo. De hecho, dado $A \in L(\mathcal{H})^{+}$inyectivo con $R(A) \neq \mathcal{H}$, consideremos el espacio de Hilbert $\mathcal{K}=\mathcal{H} \oplus \mathcal{H}$, el subespacio $\mathcal{S}=\mathcal{H} \oplus\{0\}$ y el operador $B \in L(\mathcal{K})^{+}$representado por

$$
B=\left(\begin{array}{cc}
A & 0 \\
0 & I
\end{array}\right)
$$


en la decomposición inducida por $\mathcal{S}$. Luego, $B(\mathcal{S})^{\perp}=(R(A) \oplus\{0\})^{\perp}=\{0\} \oplus \mathcal{H}$ y $\mathcal{S}+B(\mathcal{S})^{\perp}=\mathcal{K}$, es decir, $(B, \mathcal{S})$ es compatible. Por otra parte, resulta claro que $\mathcal{S}$ no es uniformemente $B$-positivo porque $R(A)$ no es cerrado.

Proposición 2.1.11. Supongamos que $B \in L(\mathcal{H})^{s}$ tiene rango cerrado y $\mathcal{S}$ es un subespacio $B$-positivo. Si $(B, \mathcal{S})$ es compatible entonces $\mathcal{S}$ es uniformemente $B$-positivo.

Demostración. Supongamos que el par $(B, \mathcal{S})$ es compatible. En primer lugar, veamos que $B(\mathcal{S})$ es un subespacio cerrado: si $\left\{y_{n}\right\}_{n \in \mathbb{N}}$ es una sucesión en $B(\mathcal{S})$ que converge a un vector $y \in \mathcal{H}$, entonces $y \in R(B)$ porque $R(B)$ es cerrado. Luego, $y=B x$ para algún $x \in \mathcal{H}$. Además, si para cada $n \in \mathbb{N}$ elegimos un $s_{n} \in \mathcal{S}$ tal que $y_{n}=B s_{n}$, vemos que

$$
y_{n}=B s_{n}=B Q s_{n}=Q^{*} B s_{n} \rightarrow Q^{*} B x=B Q x,
$$

i.e. $y=B Q x \in B(\mathcal{S})$. Por lo tanto, $B(\mathcal{S})$ es cerrado. Por su parte, por la Proposición 1.1.18, de la identidad $\mathcal{H}=\mathcal{S}+B(\mathcal{S})^{\perp}$ se desprende que $\mathcal{S}^{\perp}+B(\mathcal{S})$ es cerrado, o equivalentemente, $R\left(G_{B, \mathcal{S}}\right)=P_{\mathcal{S}}(B(\mathcal{S}))$ es cerrado.

Como $\mathcal{S}$ es $B$-positivo, podemos afirmar que $G_{B, \mathcal{S}} \in L(\mathcal{H})^{+}$y, ya que los vectores de $\mathcal{N}$ son $B$-neutros, $\mathcal{N}=\{0\}$. Luego, existe un $\alpha>0$ tal que $G_{B, \mathcal{S}} \geq \alpha P_{\mathcal{S}}$ i.e. $\mathcal{S}$ es uniformemente $B$-positivo.

\subsection{2. $\quad$ El conjunto $\mathcal{P}(B, \mathcal{S})$}

Sean $\mathcal{S}$ un subespacio cerrado de $\mathcal{H}$ y $B \in L(\mathcal{H})^{s}$ tales que $(B, \mathcal{S})$ es compatible. Ya que $\mathcal{H}=\mathcal{S} \dot{+}\left(B(\mathcal{S})^{\perp} \ominus \mathcal{N}\right)$, consideremos la proyección oblicua

$$
P_{B, \mathcal{S}}:=P_{\mathcal{S} / / B(\mathcal{S})^{\perp} \ominus \mathcal{N}}
$$

Notemos que $R\left(P_{B, \mathcal{S}}\right)=\mathcal{S}$ y $N\left(P_{B, \mathcal{S}}\right) \subseteq B(\mathcal{S})^{\perp}$. Luego, por el Lema 2.1.1, $P_{B, \mathcal{S}} \in \mathcal{P}(B, \mathcal{S})$. Además,

Proposición 2.1.12. El conjunto $\mathcal{P}(B, \mathcal{S})$ es una variedad afín que puede parametrizarse como

$$
\mathcal{P}(B, \mathcal{S})=P_{B, \mathcal{S}}+L\left(\mathcal{S}^{\perp}, \mathcal{N}\right),
$$

donde $L\left(\mathcal{S}^{\perp}, \mathcal{N}\right)$ está identificado con el subespacio de $L(\mathcal{H})$ correspondiente.

Demostración. Dada $Q \in \mathcal{P}(B, \mathcal{S})$, consideremos el operador $Z=Q-P_{B, \mathcal{S}} \in L(\mathcal{H})$. Notemos que $Z$ es $B$-autoadjunto y $\mathcal{S} \subseteq N(Z)$. De hecho, si $x \in \mathcal{S}$ entonces $Z x=Q x-P_{B, \mathcal{S}} x=x-x=0$. Por su parte, $R(Z) \subseteq \mathcal{S}$ y, si $y \in R(Z)$ luego

$$
B y \in R(B Z)=R\left(Z^{*} B\right) \subseteq R\left(Z^{*}\right)=N(Z)^{\perp} \subseteq \mathcal{S}^{\perp} .
$$

Por lo tanto, $R(Z) \subseteq \mathcal{S} \cap B(\mathcal{S})^{\perp}=\mathcal{N}$. Podemos identificar entonces - quitando al núcleo del dominio y restringiendo el codominio a la imagen - a $Z$ con un operador en $L\left(\mathcal{S}^{\perp}, \mathcal{N}\right)$ y

$$
Q=P_{B, \mathcal{S}}+Z \in P_{B, \mathcal{S}}+L\left(\mathcal{S}^{\perp}, \mathcal{N}\right) .
$$

Recíprocamente, dado $Z^{\prime} \in L\left(\mathcal{S}^{\perp}, \mathcal{N}\right)$, identifiquémoslo con el operador $Z \in L(\mathcal{H})$ definido por $Z x=Z^{\prime} x$ si $x \in \mathcal{S}^{\perp}$, y $Z s=0$ si $s \in \mathcal{S}$. Luego, $\mathcal{S} \subseteq N(Z)$ y $R(Z) \subseteq \mathcal{N}$, lo que implica que $Z^{2}=0$ y $B Z=0$.

Si consideramos el operador $Q=P_{B, \mathcal{S}}+Z \in L(\mathcal{H})$, resulta que $Q^{2}=Q$ y $B Q=B P_{B, \mathcal{S}}+$ $B Z=B P_{B, \mathcal{S}}$. Entonces $Q^{*} B=P_{B, \mathcal{S}}^{*} B=B P_{B, \mathcal{S}}=B Q$, es decir, la proyección $Q$ es $B$ autoadjunta. Como además $R(Q) \subseteq \mathcal{S}+\mathcal{N}=\mathcal{S}$, tenemos que $Q \in \mathcal{P}(B, \mathcal{S})$. 
Corolario 2.1.13. Si $(B, \mathcal{S})$ es compatible, $\mathcal{P}(B, \mathcal{S})=\left\{P_{B, \mathcal{S}}\right\}$ si y sólo si $\mathcal{N}=\{0\}$.

También como corolario de la proposición anterior, probaremos que la proyección $P_{B, \mathcal{S}}$ es la más próxima al operador identidad (con respecto a la norma de $\mathcal{H}$ ) entre todas las proyecciones del conjunto $\mathcal{P}(B, \mathcal{S})$.

Proposición 2.1.14. Sean $\mathcal{S}$ un subespacio cerrado de $\mathcal{H}$ y $B \in L(\mathcal{H})^{s}$ tales que $(B, \mathcal{S})$ es compatible. Si $x \in \mathcal{H}$, entonces $\left(I-P_{B, \mathcal{S}}\right) x$ es el único elemento de norma mínima en el conjunto

$$
\{(I-Q) x: Q \in \mathcal{P}(B, \mathcal{S})\}
$$

Demostración. Dado $x \in \mathcal{H}$, consideremos su descomposición ortogonal $x=s+y$ con $s \in \mathcal{S}$ e $y \in \mathcal{S}^{\perp}$. Notemos que $\left(I-P_{B, \mathcal{S}}\right) y \in N\left(P_{B, \mathcal{S}}\right) \subseteq \mathcal{N}^{\perp}$. Luego, por la Proposición 'refparamet 1, si $Q \in \mathcal{P}(B, \mathcal{S})$ existe un operador $Z \in L\left(\mathcal{S}^{\perp}, \mathcal{N}\right)$ tal que $Q=P_{B, \mathcal{S}}+Z$, y además

$$
\begin{aligned}
\|(I-Q) x\|^{2} & =\|(I-Q) y\|^{2}=\left\|\left(I-P_{B, \mathcal{S}}\right) y-Z y\right\|^{2}=\left\|\left(I-P_{B, \mathcal{S}}\right) y\right\|^{2}+\|Z y\|^{2}= \\
& =\left\|\left(I-P_{B, \mathcal{S}}\right) x\right\|^{2}+\|Z y\|^{2}
\end{aligned}
$$

ya que $Z y \in \mathcal{N}$. Por lo tanto, $\|(I-Q) x\| \geq\left\|\left(I-P_{B, \mathcal{S}}\right) x\right\|$ para todo $Q \in \mathcal{P}(B, \mathcal{S})$. Por otro lado, si $(I-Q) x$ tiene norma mínima entre los vectores de (2.1.2), entonces $\|(I-Q) x\|=\left\|\left(I-P_{B, \mathcal{S}}\right) x\right\|$ y, en consecuencia, $Z y=0$. Por lo tanto, $(I-Q) x=(I-Q) y=\left(I-P_{B, \mathcal{S}}\right) y-Z y=\left(I-P_{B, \mathcal{S}}\right) y=$ $\left(I-P_{B, \mathcal{S}}\right) x$.

Corolario 2.1.15. Si $(B, \mathcal{S})$ es compatible entonces

$$
\left\|I-P_{B, \mathcal{S}}\right\|=\min \{\|I-Q\|: Q \in \mathcal{P}(B, \mathcal{S})\} .
$$

En [20, Proposición 3.5], G. Corach, A. Maestripieri y D. Stojanoff probaron que, dado $A \in L(\mathcal{H})^{+}$, toda proyección $A$-autoadjunta puede escribirse como la suma de la proyección minimal sobre $\mathcal{S} \ominus \mathcal{N}$ y una proyección oblicua adecuada con rango $\mathcal{N}$. Dicho resultado puede generalizarse, mutatis mutandis, a operadores autoadjuntos. A continuación, presentamos una versión simplificada del mismo.

Proposición 2.1.16. Sean $\mathcal{S}$ un subespacio cerrado de $\mathcal{H}$ y $B \in L(\mathcal{H})^{s}$. Si $(B, \mathcal{S})$ es compatible y $Q \in \mathcal{P}(B, \mathcal{S})$ entonces

$$
Q=P_{B, \mathcal{S} \ominus \mathcal{N}}+P_{\mathcal{N} / /(\mathcal{S} \ominus \mathcal{N}+N(Q))}
$$

Demostración. Supongamos que $Q \in \mathcal{P}(B, \mathcal{S})$ y sea $E=P_{\mathcal{N}} Q$. Es fácil ver que $R(E)=\mathcal{N}$ y además, como $P_{\mathcal{N}} Q=P_{\mathcal{N}}$, se tiene que $E \in \mathcal{Q}$. Por su parte, como $E$ es el producto de dos

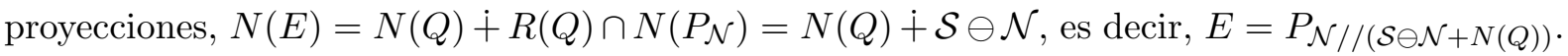
Notemos que $E$ es $B$-autoadjunta porque $B E=0$.

Luego, si $P=Q-E$, se sigue que $P$ es $B$-autoadjunto ya que $Q$ y $E$ lo son. Por otro lado, $P^{2}=P$ porque $Q E=E=E Q$. Finalmente, notemos que $R(P)=\mathcal{S} \ominus \mathcal{N}$ y, por el Corolario

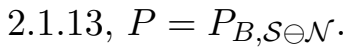




\subsubsection{Representación matricial inducida por un subespacio compatible}

Dado un operador $T \in L(\mathcal{H})$, todo subespacio cerrado $\mathcal{S}$ de $\mathcal{H}$ induce una representación de $B$ como una matriz de $2 \times 2$, de acuerdo con la descomposición $\mathcal{H}=\mathcal{S} \oplus \mathcal{S}^{\perp}$. Más precisamente, si $P=P_{\mathcal{S}}$ es la proyección ortogonal sobre $\mathcal{S}, T$ puede ser representado como

$$
T=\left(\begin{array}{ll}
a & b \\
c & d
\end{array}\right)
$$

siendo $a=\left.P T P\right|_{\mathcal{S}}: \mathcal{S} \rightarrow \mathcal{S}, b=\left.P T(I-P)\right|_{\mathcal{S}^{\perp}}: \mathcal{S}^{\perp} \rightarrow \mathcal{S}, c=\left.(I-P) T P\right|_{\mathcal{S}}: \mathcal{S} \rightarrow \mathcal{S}^{\perp}$ y $d=\left.(I-P) T(I-P)\right|_{\mathcal{S}^{\perp}}: \mathcal{S}^{\perp} \rightarrow \mathcal{S}^{\perp}$ operadores acotados. En estos párrafos reescribiremos algunos de los resultados anteriores utilizando esta representación.

Notemos que, si $Q$ es una proyección con rango $\mathcal{S}$, entonces $Q$ puede representarse como

$$
Q=\left(\begin{array}{cc}
I & x \\
0 & 0
\end{array}\right), \quad \text { para algún } x \in L\left(\mathcal{S}^{\perp}, \mathcal{S}\right)
$$

En particular, $P=\left(\begin{array}{ll}I & 0 \\ 0 & 0\end{array}\right)$. Por otra parte, si $B \in L(\mathcal{H})^{s}$, la representación matricial de $B$ es la forma

$$
B=\left(\begin{array}{cc}
a & b \\
b^{*} & c
\end{array}\right)
$$

siendo $a=\left.P_{\mathcal{S}} B\right|_{\mathcal{S}}: \mathcal{S} \rightarrow \mathcal{S}, b=\left.P_{\mathcal{S}} B\right|_{\mathcal{S}^{\perp}}: \mathcal{S}^{\perp} \rightarrow \mathcal{S}$ y $c=\left.P_{\mathcal{S}^{\perp}} B\right|_{\mathcal{S}^{\perp}}: \mathcal{S}^{\perp} \rightarrow \mathcal{S}^{\perp}$.

En primer lugar, notemos que la proyección $Q$, dada por la Ec. (2.1.3), es $B$-autoadjunta si y sólo si $a x=b$. Es decir,

Teorema 2.1.17. Dados $B \in L(\mathcal{H})^{s}$ y un subespacio cerrado $\mathcal{S}$ de $\mathcal{H}$, consideremos la representación matricial de $B$ inducida por $\mathcal{S}, B=\left(\begin{array}{cc}a & b \\ b^{*} & c\end{array}\right)$. Luego, son equivalentes:

1. el par $(B, \mathcal{S})$ es compatible;

2. $R(b) \subseteq R(a)$;

3. la ecuación $a x=b$ admite (al menos) una solución en $L\left(\mathcal{S}^{\perp}, \mathcal{S}\right)$.

Por otra parte, si $s \in \mathcal{S}$ entonces $B s=a s+b^{*} s, a s \in \mathcal{S}$ y $b^{*} s \in \mathcal{S}^{\perp}$. Luego, $B s \in \mathcal{S}^{\perp}$ si y sólo si $s \in N(a)$. Por lo tanto, la parte $B$-isotrópica de $\mathcal{S}$ coincide con el núcleo de $a \in L(\mathcal{S})$, i.e.

$$
\mathcal{N}=N(a)
$$

Proposición 2.1.18. Sean $B \in L(\mathcal{H})^{s}$ y $\mathcal{S}$ un subespacio cerrado de $\mathcal{H}$ tales que $(B, \mathcal{S})$ es compatible. Si $B=\left(\begin{array}{cc}a & b \\ b^{*} & c\end{array}\right)$ es la representación matricial de $B$ inducida por $\mathcal{S}$, tenemos que:

1. Si $d \in L\left(\mathcal{S}^{\perp}, \mathcal{S}\right)$ es la solución reducida de $a x=b$ entonces la representación matricial de $P_{B, \mathcal{S}}$ inducida por $\mathcal{S}$ es $P_{B, \mathcal{S}}=\left(\begin{array}{cc}I & d \\ 0 & 0\end{array}\right)$. 
2. La parametrización de la Proposición 2.1.12 puede representarse matricialmente mediante

$$
Q(z)=\left(\begin{array}{ccc}
I & 0 & d \\
0 & I & z \\
0 & 0 & 0
\end{array}\right) \quad \text { para } z \in L\left(\mathcal{S}^{\perp}, \mathcal{N}\right)
$$

de acuerdo a la descomposición ortogonal $\mathcal{H}=(\mathcal{S} \ominus \mathcal{N}) \oplus \mathcal{N} \oplus \mathcal{S}^{\perp}$.

Demostración. En primer lugar, recordemos que $N\left(P_{B, \mathcal{S}}\right)=B(\mathcal{S})^{\perp} \ominus \mathcal{N}$. Como $Q=\left(\begin{array}{cc}I & d \\ 0 & 0\end{array}\right)$ es una proyección $B$-autoadjunta con rango $\mathcal{S}$, sólo resta probar que $N(Q) \subseteq B(\mathcal{S})^{\perp} \ominus \mathcal{N}$. Por el Lema 2.1.1, tenemos que $N(Q) \subseteq B(\mathcal{S})^{\perp}$. Además, si $x \in N(Q)$ y $x=x_{1}+x_{2}$ con $x_{1} \in \mathcal{S}$ y $x_{2} \in \mathcal{S}^{\perp}$, vemos que $x_{1}+d x_{2}=0$. Luego, para todo $n \in \mathcal{N}$,

$$
\langle x, n\rangle=\left\langle x_{1}, n\right\rangle=\left\langle-d x_{2}, n\right\rangle=0
$$

ya que $R(d) \subseteq \overline{R(a)}=N(a)^{\perp}=\mathcal{N}^{\perp}$ por ser $d$ la solución reducida. Por lo tanto, $N(Q) \subseteq$ $B(\mathcal{S})^{\perp} \ominus \mathcal{N}$ y, en consecuencia, $Q=P_{B, \mathcal{S}}$.

Por su parte, si $Q \in \mathcal{P}(B, \mathcal{S})$, existe $z \in L\left(\mathcal{S}^{\perp}, \mathcal{N}\right)$ tal que $Q=P_{B, \mathcal{S}}+z$. Como $R\left(P_{B, \mathcal{S}}\right) \subseteq$ $\mathcal{S} \ominus \mathcal{N}$, es inmediato que la representación matricial de $Q$ de acuerdo a la descomposición ortogonal $\mathcal{H}=(\mathcal{S} \ominus \mathcal{N}) \oplus \mathcal{N} \oplus \mathcal{S}^{\perp}$ es

$$
Q=P_{B, \mathcal{S}}+z=\left(\begin{array}{ccc}
I & 0 & d \\
0 & I & 0 \\
0 & 0 & 0
\end{array}\right)+\left(\begin{array}{ccc}
0 & 0 & 0 \\
0 & 0 & z \\
0 & 0 & 0
\end{array}\right)=\left(\begin{array}{ccc}
I & 0 & d \\
0 & I & z \\
0 & 0 & 0
\end{array}\right)
$$

Observación 2.1.19. Si $B=\left(\begin{array}{cc}a & b \\ b^{*} & c\end{array}\right)$ es la representación matricial de $B$ inducida por $\mathcal{S}$, la solución reducida $d \in L\left(\mathcal{S}^{\perp}, \mathcal{S}\right)$ de $a x=b$ es:

$$
d=a^{\dagger} b
$$

A pesar de que $a^{\dagger}$ es acotado si y sólo si $R(a)$ es un subespacio cerrado, si el par $(B, \mathcal{S})$ es compatible, el producto $a^{\dagger} b$ se mantiene acotado. Luego, $d=a^{\dagger} b$ es solución de la ecuación $a x=b$ y cumple $R(d) \subseteq R\left(a^{\dagger}\right) \subseteq \overline{R\left(a^{*}\right)}$, es decir, esta es la solución reducida de la ecuación. Por lo tanto, la representación matricial de $P_{B, \mathcal{S}}$ inducida por $\mathcal{S}$ es:

$$
P_{B, \mathcal{S}}=\left(\begin{array}{cc}
I & a^{\dagger} b \\
0 & 0
\end{array}\right)
$$

\subsection{Condiciones necesarias y suficientes para la compatibilidad}

Dado $B \in L(\mathcal{H})^{s}$, sea $B=J A$ su descomposición polar, con $A \in L(\mathcal{H})^{+}$y $J=J^{*}=J^{-1}$. La siguiente proposición presenta algunas condiciones, en términos de $A$, que resultan necesarias para la compatibilidad. Las implicaciones $2 . \Rightarrow 3 . \Rightarrow 4$. $\Leftrightarrow 5$. fueron probadas en [21, Proposición $3.4]$. 
Proposición 2.2.1. Dado un operador $B \in L(\mathcal{H})^{s}$ con descomposición polar $B=J A$ consideremos las siguientes condiciones:

1. El par $(B, \mathcal{S})$ es compatible,

2. $A(\mathcal{S})$ es cerrado en $R(A)$ i.e. $\overline{A(\mathcal{S})} \cap R(A)=A(\mathcal{S})$,

3. $A^{1 / 2}(\mathcal{S})$ es cerrado en $R\left(A^{1 / 2}\right)$,

4. $\mathcal{S}+N(B)$ es cerrado.

5. $P_{\overline{R(B)}}(\mathcal{S})$ es cerrado.

Entonces, $1 . \Rightarrow 2 . \Rightarrow 3 . \Rightarrow 4$. $\Leftrightarrow 5$.

Demostración. 1. $\Rightarrow$ 2. : Supongamos que $(B, \mathcal{S})$ es compatible y sea $Q \in \mathcal{P}(B, \mathcal{S})$. Con un argumento similar al utilizado en la Proposición 2.1.11 se tiene que $B(\mathcal{S})$ es cerrado en $R(B)$ : dado $y \in \overline{B(\mathcal{S})} \cap R(B)$, sea $\left\{s_{n}\right\}_{n \in \mathbb{N}}$ una sucesión en $\mathcal{S}$ tal que $B\left(s_{n}\right) \rightarrow y$. Luego, $y=B x$ para algún $x \in \mathcal{H}$ y $B s_{n}=B Q s_{n}=Q^{*} B s_{n} \rightarrow Q^{*} B x=B Q x$. Por lo tanto, $y=B Q x \in B(\mathcal{S})$.

Además, $B(\mathcal{S})$ es cerrado en $R(B)$ si y sólo si $A(\mathcal{S})$ es cerrado en $R(A)$ : si $B(\mathcal{S})$ es cerrado en $R(B)$ entonces $B(\mathcal{S})=\overline{B(\mathcal{S})} \cap R(B)$, es decir, $J(A(\mathcal{S}))=\overline{J A(\mathcal{S})} \cap J(R(A))=J(\overline{A(\mathcal{S})} \cap R(A))$. Por lo tanto, $A(\mathcal{S})=\overline{A(\mathcal{S})} \cap R(B)$ i.e. $A(\mathcal{S})$ es cerrado en $R(A)$. La recíproca es análoga.

2. $\Rightarrow$ 3: Si $y \in \overline{A^{1 / 2}(\mathcal{S})} \cap R\left(A^{1 / 2}\right)$ entonces $y=A^{1 / 2} x$ para algún $x \in \mathcal{H}$ y además existe una sucesión $\left\{s_{n}\right\}_{n \in \mathbb{N}}$ en $\mathcal{S}$ tal que $A^{1 / 2} s_{n} \rightarrow A^{1 / 2} x$. Luego, $A s_{n} \rightarrow A x$ y consecuentemente $A x \in$ $\overline{A(\mathcal{S})} \cap R(A)=A(\mathcal{S})$. Por lo tanto, $y \in A^{-1 / 2}(A(\mathcal{S}))=\left(A^{1 / 2}(\mathcal{S})+N(A)\right) \cap R\left(A^{1 / 2}\right)=A^{1 / 2}(\mathcal{S})$. 3. $\Rightarrow$ 4: $\mathrm{Si} A^{1 / 2}(\mathcal{S})$ es cerrado en $R\left(A^{1 / 2}\right)$ entonces

$$
\mathcal{S}+N(A)=A^{-1 / 2}\left(A^{1 / 2}(\mathcal{S})\right)=A^{-1 / 2}\left(\overline{A^{1 / 2}(\mathcal{S})} \cap R\left(A^{1 / 2}\right)\right)=A^{-1 / 2}\left(\overline{A^{1 / 2}(\mathcal{S})}\right)
$$

es un subespacio cerrado.

4. $\Rightarrow$ 5: Es un caso particular de la Proposición 1.1.18.

A continuación, mostraremos que, dados $B \in L(\mathcal{H})^{s}$ y un subespacio cerrado $\mathcal{S}$ de $\mathcal{H}$, la existencia de proyecciones $B$-autoadjuntas con rango $\mathcal{S}$ no depende del subespacio $\mathcal{S}$ en su totalidad, sino del subespacio que resulta de proyectar a $\mathcal{S}$ sobre la clausura del rango de $B$.

Proposición 2.2.2. Sea $B \in L(\mathcal{H})^{s}$ y consideremos un subespacio cerrado $\mathcal{S}$ de $\mathcal{H}$. Luego, $(B, \mathcal{S})$ es compatible si y sólo si $P_{\overline{R(B)}}(\mathcal{S})$ es cerrado y $\left(B, P_{\overline{R(B)}}(\mathcal{S})\right)$ es compatible.

Demostración. Recordemos que $B=B P_{\overline{R(B)}}$. Luego, como $\mathcal{S}+N(B)=P_{\overline{R(B)}}(\mathcal{S}) \oplus N(B)$ y $N(B) \subseteq B^{-1}\left(\mathcal{S}^{\perp}\right)=B(\mathcal{S})^{\perp}$, se tiene que

$$
\mathcal{S}+B(\mathcal{S})^{\perp}=\mathcal{S}+N(B)+B(\mathcal{S})^{\perp}=P_{\overline{R(B)}}(\mathcal{S})+B(\mathcal{S})^{\perp}=P_{\overline{R(B)}}(\mathcal{S})+B\left(P_{\overline{R(B)}}(\mathcal{S})\right)^{\perp} .
$$

Por lo tanto, $(B, \mathcal{S})$ es compatible si y sólo si $P_{\overline{R(B)}}(\mathcal{S})$ es cerrado y $\left(B, P_{\overline{R(B)}}(\mathcal{S})\right)$ es compatible.

Mas aún, si $B \in L(\mathcal{H})^{s}$ y $\mathcal{S}$ es un subespacio cerrado de $\mathcal{H}$, para estudiar la compatibilidad de $(B, \mathcal{S})$ podemos restringir tanto el subespacio $\mathcal{S}$ como el operador $B$ al subespacio $\mathcal{K}=\overline{R(B)}$. 
Observación 2.2.3. Dado $B \in L(\mathcal{H})^{s}$, sea $\mathcal{K}=\overline{R(B)}$ y consideremos el operador

$$
C=\left.B\right|_{\mathcal{K}} \in L(\mathcal{K})^{s} .
$$

Luego, $(B, \mathcal{S})$ es compatible si y sólo si $P_{\overline{R(B)}}(\mathcal{S})$ es cerrado y $\left(C, P_{\overline{R(B)}}(\mathcal{S})\right)$ es compatible.

De hecho, aplicando la proposición anterior, podemos suponer que $\mathcal{S} \subseteq \mathcal{K}$. Entonces, la afirmación es una consecuencia directa de la demostración anterior, notando que

$$
B^{-1}\left(\mathcal{S}^{\perp}\right)=C^{-1}(\mathcal{K} \ominus \mathcal{S})+N(B)
$$

y que $\mathcal{K} \ominus \mathcal{S}$ es el complemento ortogonal de $\mathcal{S}$ en el espacio de Hilbert $\mathcal{K}$.

\subsubsection{Descomposición de rangos de operadores}

En [21], G. Corach, A. Maestripieri y D. Stojanoff mostraron que, dado un operador $A \in$ $L(\mathcal{H})^{+}$y un subespacio cerrado $\mathcal{S}$ del espacio de Hilbert $\mathcal{H}$, el par $(A, \mathcal{S})$ es compatible si y sólo si el rango de $A^{1 / 2}$ admite una descomposición ortogonal en términos del subespacio $\mathcal{M}=\overline{A^{1 / 2}(\mathcal{S})}$ :

$$
R\left(A^{1 / 2}\right)=\mathcal{M} \cap R\left(A^{1 / 2}\right) \oplus \mathcal{M}^{\perp} \cap R\left(A^{1 / 2}\right) .
$$

Dado un operador autoadjunto $B \in L(\mathcal{H})^{s}$, cuya descomposición polar está dada por $B=J A$ con $A \in L(\mathcal{H})^{+}$y $J=J^{*}=J^{-1}$, la siguiente proposición relaciona la compatibilidad del par $(B, \mathcal{S})$ con ciertas descomposiciones (en suma directa) de $R(A)$ y $R\left(A^{1 / 2}\right)$.

Proposición 2.2.4. Dado $B \in L(\mathcal{H})^{s}$ consideremos su descomposición polar $B=J A$, con $A \in L(\mathcal{H})^{+}$y $J=J^{*}=J^{-1}$. Si $\mathcal{S}$ es un subespacio cerrado de $\mathcal{H}$, las siguientes condiciones son equivalentes:

1. $\operatorname{El} \operatorname{par}(B, \mathcal{S})$ es compatible;

2. $R(A)=A(\mathcal{S}) \dot{+} J(\mathcal{S})^{\perp} \cap R(A)$;

3. $R\left(A^{1 / 2}\right)=A^{1 / 2}(\mathcal{S})+J\left(A^{1 / 2}(\mathcal{S})\right)^{\perp} \cap R\left(A^{1 / 2}\right)$.

Demostración. 1. $\Leftrightarrow$ 2.: $\mathrm{Si}(B, \mathcal{S})$ es compatible entonces $\mathcal{H}=\mathcal{S}+B^{-1}\left(\mathcal{S}^{\perp}\right)$. Aplicando $A$ a ambos lados de esta igualdad, se tiene que $R(A)=A(\mathcal{S})+J\left(\mathcal{S}^{\perp}\right) \cap R(A)$. Además, la suma es directa, ya que

$$
A(\mathcal{S}) \cap J\left(\mathcal{S}^{\perp}\right)=J\left(B(\mathcal{S}) \cap S^{\perp}\right) \subseteq J\left(\overline{B(\mathcal{S})} \cap S^{\perp}\right)=J\left(\left(\mathcal{S}+B(\mathcal{S})^{\perp}\right)^{\perp}\right)=J\left(\mathcal{H}^{\perp}\right)=\{0\} .
$$

Recíprocamente, supongamos que $R(A)=A(\mathcal{S})+J\left(\mathcal{S}^{\perp}\right) \cap R(A)$. Luego,

$$
\mathcal{H}=A^{-1}(R(A))=A^{-1}\left(A(\mathcal{S}) \dot{+} J\left(\mathcal{S}^{\perp}\right) \cap R(A)\right),
$$

y, dado $x \in \mathcal{H}$, existen $s \in \mathcal{S}$ y $z \in J\left(\mathcal{S}^{\perp}\right) \cap R(A)$ tales que $A x=A s+z$. Si $w \in \mathcal{H}$ es tal que $A w=z$, luego $A x=A s+A w=A(s+w)$. Por lo tanto, existe un $n \in N(A)$ tal que $x=s+w+n$, es decir, $x=(s+n)+w \in \mathcal{S}+N(A)+A^{-1}\left(J\left(\mathcal{S}^{\perp}\right)\right)=\mathcal{S}+B^{-1}\left(\mathcal{S}^{\perp}\right)$. Entonces, como $x \in \mathcal{H}$ era arbitrario, $\mathcal{H} \subseteq \mathcal{S}+B^{-1}\left(\mathcal{S}^{\perp}\right)$, o equivalentemente, $(B, \mathcal{S})$ es compatible.

La demostración de la equivalencia 1. $\Leftrightarrow$ 3. es análoga a la anterior. 
Es importante remarcar que, en la descomposición del item 3. de la proposición anterior, no hay ortogonalidad en el sentido del espacio de Hilbert $\mathcal{H}$ sino que los subespacios son $J$ ortogonales en el espacio de Krein que induce el operador $J$ sobre $\mathcal{H}$. Lo mismo ocurre con la descomposición de $R\left(A^{1 / 2}\right)$ del siguiente corolario.

Corolario 2.2.5. Dados un subespacio cerrado $\mathcal{S}$ de $\mathcal{H}$ y $B \in L(\mathcal{H})^{s}$, consideremos el subespacio $\mathcal{M}=\overline{A^{1 / 2}(\mathcal{S})}$. Entonces, $(B, \mathcal{S})$ es compatible si y sólo si $A^{1 / 2}(\mathcal{S})$ es cerrado en $R\left(A^{1 / 2}\right)$ y

$$
R\left(A^{1 / 2}\right)=\mathcal{M} \cap R\left(A^{1 / 2}\right) \dot{+} J(\mathcal{M})^{\perp} \cap R\left(A^{1 / 2}\right) .
$$

Demostración. Es consecuencia de las Proposiciones 2.2.1 y 2.2.4.

Corolario 2.2.6. Si $(B, \mathcal{S})$ es compatible entonces $\mathcal{M}=\overline{A^{1 / 2}(\mathcal{S})}$ es un subespacio $J$-no degenerado del espacio de Krein que resulta de dotar a $\mathcal{H}$ con la simetría fundamental $J$.

Demostración. Por el corolario anterior,

$$
\mathcal{H}=\overline{R\left(A^{1 / 2}\right)} \oplus N\left(A^{1 / 2}\right) \subseteq \overline{\mathcal{M}+J(\mathcal{M})^{\perp}}+N\left(A^{1 / 2}\right)=\overline{\mathcal{M}+J(\mathcal{M})^{\perp}},
$$

ya que $N\left(A^{1 / 2}\right) \subseteq J(\mathcal{M})^{\perp} \subseteq \mathcal{M}+J(\mathcal{M})^{\perp} \subseteq \overline{\mathcal{M}+J(\mathcal{M})^{\perp}}$. Luego, como consecuencia del Corolario 1.2.30, $\mathcal{M}$ es $J$-no degenerado.

\subsubsection{Compatibilidad y ángulos entre subespacios}

Dados un subespacio cerrado $\mathcal{S}$ de $\mathcal{H}$ y un operador $B \in L(\mathcal{H})^{s}$, la compatibilidad del par $(B, \mathcal{S})$ también puede interpretarse en términos del ángulo entre ciertos subespacios cerrados, es decir, el par $(B, \mathcal{S})$ es compatible si y sólo si el ángulo entre ciertos subespacios no es nulo. Las definiciones de ángulo y ángulo mínimo entre subespacios cerrados, así como sus propiedades, pueden consultarse en los preliminares.

Teorema 2.2.7. Sean $B \in L(\mathcal{H})^{s}$ y $\mathcal{S}$ un subespacio cerrado de $\mathcal{H}$. Entonces, $(B, \mathcal{S})$ es compatible si y sólo si $c_{0}\left(\mathcal{S}^{\perp}, \overline{B(\mathcal{S})}\right)<1$.

La prueba que presentamos a continuación fue realizada originalmente en [20, Teorema 2.15], para el caso en que el operador es (semidefinido) positivo. Los argumentos utilizados en la misma siguen siendo válidos para operadores autoadjuntos.

Demostración. Supongamos que $(B, \mathcal{S})$ es compatible. Luego, $\mathcal{H}=\mathcal{S}+B(\mathcal{S})^{\perp}$ y, tomando el complemento ortogonal, tenemos que $\mathcal{S}^{\perp} \cap \overline{B(\mathcal{S})}=\{0\}$. Entonces, $c_{0}(\mathcal{S}, \overline{B(\mathcal{S})})=c(\mathcal{S}, \overline{B(\mathcal{S})})$. Además, por la Proposición 1.1.18, la igualdad $\mathcal{H}=\mathcal{S}+B(\mathcal{S})^{\perp}$ implica que el subespacio $\mathcal{S}+\overline{B(\mathcal{S})}$ es cerrado, o equivalentemente, $c(\mathcal{S}, \overline{B(\mathcal{S})})<1$. Por lo tanto, $c_{0}(\mathcal{S}, \overline{B(\mathcal{S})})<1$.

Recíprocamente, si $c_{0}(\mathcal{S}, \overline{B(\mathcal{S})})<1$, tenemos que $c(\mathcal{S}, \overline{B(\mathcal{S})})<1$ y $\mathcal{S} \cap \overline{B(\mathcal{S})}=\{0\}$. Entonces, utilizando una vez más la Proposición 1.1.18, concluimos que $\mathcal{S}+B(\mathcal{S})^{\perp}$ es cerrado, y además,

$$
\mathcal{S}+B(\mathcal{S})^{\perp}=\overline{\mathcal{S}+B(\mathcal{S})^{\perp}}=(\mathcal{S} \cap \overline{B(\mathcal{S})})^{\perp}=\{0\}^{\perp}=\mathcal{H},
$$

es decir, $(B, \mathcal{S})$ es compatible.

Proposición 2.2.8. Sean $B \in L(\mathcal{H})^{s}$ un operador inyectivo y $\mathcal{S}$ un subespacio cerrado de $\mathcal{H}$. Entonces, $(B, \mathcal{S})$ es compatible si y sólo si $c_{0}\left(\mathcal{S}, B(\mathcal{S})^{\perp}\right)<1$. 
Demostración. Si $(B, \mathcal{S})$ es compatible, el subespacio $\mathcal{S}+B(\mathcal{S})^{\perp}$ es cerrado, o equivalentemente $c\left(\mathcal{S}, B(\mathcal{S})^{\perp}\right)<1$. Además, por la Proposición 2.1.3, sabemos que $\mathcal{S} \cap B(\mathcal{S})^{\perp}=\mathcal{S} \cap N(B)=\{0\}$, por lo que $c_{0}\left(\mathcal{S}, B(\mathcal{S})^{\perp}\right)=c\left(\mathcal{S}, B(\mathcal{S})^{\perp}\right)<1$.

Recíprocamente, si $c_{0}\left(\mathcal{S}, B(\mathcal{S})^{\perp}\right)<1$ entonces $\mathcal{S}+B(\mathcal{S})^{\perp}$ es cerrado y $\mathcal{S} \cap B^{-1}\left(\mathcal{S}^{\perp}\right)=\{0\}$. Como $B$ es inyectivo, $B\left(\mathcal{S} \cap B^{-1}\left(\mathcal{S}^{\perp}\right)\right)=B(\mathcal{S}) \cap B\left(B^{-1}\left(\mathcal{S}^{\perp}\right)\right)=B(\mathcal{S}) \cap\left(\mathcal{S}^{\perp} \cap R(B)\right)=B(\mathcal{S}) \cap \mathcal{S}^{\perp}$. Por lo tanto, $B(\mathcal{S}) \cap S^{\perp}=\{0\}$ y $\mathcal{H}=\left(S^{\perp} \cap B(\mathcal{S})\right)^{\perp}=\overline{\mathcal{S}+B(\mathcal{S})^{\perp}}=\mathcal{S}+B(\mathcal{S})^{\perp}$.

Corolario 2.2.9. Sea $B \in L(\mathcal{H})^{s}$ y consideremos un subespacio cerrado $\mathcal{S}$ de $\mathcal{H}$. Luego, $(B, \mathcal{S})$ es compatible si y sólo si $P_{\overline{R(B)}}(\mathcal{S})$ es cerrado y $c_{0}\left(P_{\overline{R(B)}}(\mathcal{S}), B(\mathcal{S})^{\perp}\right)<1$.

Demostración. Sea $\mathcal{K}=\overline{R(B)}$ y consideremos el operador $C=\left.B\right|_{\mathcal{K}} \in L(\mathcal{K})^{s}$. Por la Observación 2.2.3, sabemos que $(B, \mathcal{S})$ es compatible si y sólo si $\mathcal{T}=P_{\mathcal{K}}(\mathcal{S})$ es cerrado y el par $(C, \mathcal{T})$ es compatible en $\mathcal{K}$. Como $C \in L(\mathcal{K})^{s}$ es inyectivo, aplicando la Proposición 2.2.8 se tiene que

$$
c_{0}\left(\mathcal{T}, C(\mathcal{T})^{\perp \mathcal{K}}\right)=\sup \left\{|\langle x, y\rangle|: x \in \mathcal{T},\|x\| \leq 1, y \in C(\mathcal{T})^{\perp \mathcal{K}},\|y\| \leq 1\right\}<1,
$$

donde el espacio de Hilbert considerado en la condición de ángulo es $\mathcal{K}$ (con la norma heredada de $\mathcal{H})$.

Veamos ahora que $c_{0}\left(\mathcal{T}, B(\mathcal{S})^{\perp}\right) \leq c_{0}\left(\mathcal{T}, C(\mathcal{T})^{\perp_{\mathcal{K}}}\right)$. En primer lugar, notemos que, como $B=B P_{\overline{R(B)}}$, tenemos que $C(\mathcal{T})=B(\mathcal{S}) \subset R(B)$ y luego $B(\mathcal{S})^{\perp}=C(\mathcal{T})^{\perp} \mathcal{K} \oplus N(B)$. Entonces, si $x \in \mathcal{T}$ con $\|x\| \leq 1$ e $y \in B(\mathcal{S})^{\perp}$ con $\|y\| \leq 1$, notemos que

$$
|\langle x, y\rangle|=\left|\left\langle x, y_{1}+y_{2}\right\rangle\right|=\left|\left\langle x, y_{1}\right\rangle\right|,
$$

siendo $y=y_{1}+y_{2}$ con $y_{1} \in C(\mathcal{T})^{\perp_{\mathcal{K}}}$ e $y_{2} \in N(B)$. Además, $\left\|y_{1}\right\| \leq\|y\| \leq 1$ y, en consecuencia, fijado $x \in \mathcal{T}$,

$$
\sup _{y \in B(\mathcal{S})^{\perp},\|y\| \leq 1}|\langle x, y\rangle| \leq \sup _{y \in C(\mathcal{T})^{\perp \mathcal{K},\|y\| \leq 1}}|\langle x, y\rangle| .
$$

Por lo tanto, $c_{0}\left(\mathcal{T}, B(\mathcal{S})^{\perp}\right) \leq c_{0}\left(\mathcal{T}, C(\mathcal{T})^{\perp \mathcal{K}}\right)<1$.

El siguiente ejemplo está basado en uno aparecido en el libro de Halmos [37, páginas 28-29]. El objetivo del mismo es mostrar que, dada una simetría $J$, existe (al menos) un subespacio $\mathcal{S}$ que es $J$-no degenerado pero el par $(J, \mathcal{S})$ no es compatible. Vale aclarar que existen ejemplos similares en la literatura, e.g. [9, Ejemplo 9.7], pero las técnicas utilizadas en los mismos son diferentes.

Ejemplo 2.2.10. Dados un espacio de Hilbert separable de dimensión infinita $\mathcal{H}$ y un subespacio cerrado $\mathcal{S}$ de $\mathcal{H}$ con $\operatorname{dim} \mathcal{S}=\operatorname{dim} \mathcal{S}^{\perp}$, consideremos la simetría $J=2 P_{\mathcal{S}}-I \in L(\mathcal{H})$.

Fijadas bases ortonormales $\left\{a_{n}\right\}_{n \in \mathbb{N}}$ de $\mathcal{S}$ y $\left\{b_{n}\right\}_{n \in \mathbb{N}}$ de $\mathcal{S}^{\perp}$, consideremos las familias ortonormales $\left\{f_{n}\right\}_{n \in \mathbb{N}}$ y $\left\{h_{n}\right\}_{n \in \mathbb{N}}$ definidas por

$$
f_{n}=\frac{1}{\sqrt{2}}\left(a_{n}+b_{n}\right) \text { y } h_{n}=\frac{1}{\sqrt{2}}\left(a_{n}-b_{n}\right) .
$$

Finalmente, definamos $g_{n}=\left(\cos \frac{1}{n}\right) f_{n}+\left(\sin \frac{1}{n}\right) h_{n}$ y consideremos el subespacio cerrado generado por $\left\{g_{n}\right\}_{n \in \mathbb{N}}, \mathcal{T}=\overline{\operatorname{span}\left\{g_{n}\right\}_{n \in \mathbb{N}}}$. En primer lugar, notemos que $(J, \mathcal{T})$ no es compatible: de hecho,

$$
J g_{n}=\left(\cos \frac{1}{n}\right) h_{n}+\left(\sin \frac{1}{n}\right) f_{n}
$$


es una base ortonormal de $J(\mathcal{T})$ y $\left\{u_{n}\right\}_{n \in \mathbb{N}}$, with $u_{n}=\left(\sin \frac{1}{n}\right) h_{n}-\left(\cos \frac{1}{n}\right) f_{n}$, es una base ortonormal de $J(\mathcal{T})^{\perp}$. Observemos que

$$
\begin{aligned}
\left\langle g_{n}, u_{n}\right\rangle & =\left\langle\left(\cos \frac{1}{n}\right) f_{n}+\left(\sin \frac{1}{n}\right) h_{n},\left(\sin \frac{1}{n}\right) h_{n}-\left(\cos \frac{1}{n}\right) f_{n}\right\rangle= \\
& \left.=\left\langle\cos \frac{1}{n}\right) f_{n},-\left(\cos \frac{1}{n}\right) f_{n}\right\rangle+\left\langle\left(\sin \frac{1}{n}\right) h_{n},\left(\sin \frac{1}{n}\right) h_{n}\right\rangle= \\
& =-\cos \frac{2}{n}
\end{aligned}
$$

y $c_{0}\left(\mathcal{T}, J(\mathcal{T})^{\perp}\right) \geq\left|\left\langle g_{n}, u_{n}\right\rangle\right|=\cos \frac{2}{n} \underset{n \rightarrow+\infty}{\longrightarrow}$, i.e. $c_{0}\left(\mathcal{T}, J(\mathcal{T})^{\perp}\right)=1$. Entonces, por la Proposición $2.2 .8,(J, \mathcal{T})$ no es compatible.

Para completar el ejemplo falta probar que $\mathcal{T}$ es un subespacio $J$-no degenerado de $\mathcal{H}$. Supongamos que $v \in \mathcal{T} \cap J(\mathcal{T})^{\perp}, v=\sum_{n=1}^{\infty} \alpha_{n} g_{n}=\sum_{n=1}^{\infty} \beta_{n} u_{n}$, con $\sum_{n=1}^{\infty}\left|\alpha_{n}\right|^{2}<\infty$, $\sum_{n=1}^{\infty}\left|\beta_{n}\right|^{2}<\infty$. Luego,

$$
\sum_{n=1}^{\infty} \alpha_{n}\left(\cos \frac{1}{n}\right) f_{n}+\sum_{n=1}^{\infty} \alpha_{n}\left(\sin \frac{1}{n}\right) h_{n}=v=\sum_{n=1}^{\infty} \beta_{n}\left(\sin \frac{1}{n}\right) h_{n}-\sum_{n=1}^{\infty} \beta_{n}\left(\cos \frac{1}{n}\right) f_{n},
$$

lo que implica que $\alpha_{n}=\beta_{n}=0$ para todo $n \in \mathbb{N}$, es decir, $v=0$. Por lo tanto, $\mathcal{T}$ es $J$-no degenerado.

\subsubsection{Proyecciones $B$-autoadjuntas para operadores de rango cerrado}

A continuación estudiaremos la compatibilidad de pares $(B, \mathcal{S})$ en los cuales $B \in C R(\mathcal{H})^{s}$, el conjunto de operadores autoadjuntos de rango cerrado. A lo largo de esta subsección, dado $B \in C R(\mathcal{H})^{s}$, su descomposición polar es $B=J A$, con $A \in C R(\mathcal{H})^{+}$y $J=J^{*}=J^{-1}$. Además, si $\mathcal{S}$ es un subespacio cerrado de $\mathcal{H}, P=P_{\mathcal{S}}$ es la proyección ortogonal sobre $\mathcal{S}$ y $\mathcal{M}=A^{1 / 2}(\mathcal{S})$.

En [19, Teorema 6.2], G. Corach et al. probaron que:

Proposición 2.2.11. Si $A \in C R(\mathcal{H})^{+}$y $\mathcal{S}$ es un subespacio cerrado de $\mathcal{H}$, entonces las siguientes condiciones son equivalentes:

1. $\operatorname{El} \operatorname{par}(A, \mathcal{S})$ es compatible;

2. $R(P A P)$ es cerrado;

3. $\mathcal{S}+N(A)$ es cerrado.

Los próximos resultados tienen por objeto generalizar este resultado.

Proposición 2.2.12. Sean $B \in C R(\mathcal{H})^{s}$ y $\mathcal{S}$ un subespacio cerrado de $\mathcal{H}$. Luego, $(B, \mathcal{S})$ es compatible si y sólo si $\mathcal{N}=\mathcal{S} \cap N(B)$ y $G_{B, \mathcal{S}}$ tiene rango cerrado.

Demostración. Recordemos que, por el Lema 2.1.8, si $(B, \mathcal{S})$ es compatible y $\mathcal{T}=\mathcal{S} \ominus \mathcal{N}$ entonces $(B, \mathcal{T})$ es compatible, $G_{B, \mathcal{S}}=G_{B, \mathcal{T}}$ y la restricción de el operador de Gram $G_{B, \mathcal{T}}$ al subespacio $\mathcal{T}, G=\left.P_{\mathcal{T}} B\right|_{\mathcal{T}}: \mathcal{T} \rightarrow \mathcal{T}$ es inyectivo.

Por su parte, como $R(B)$ es cerrado, la Proposición 2.2.1 asegura que $B(\mathcal{T})$ es cerrado, mientras que de la identidad $\mathcal{H}=\mathcal{T}+B(\mathcal{T})^{\perp}$ se tiene que $\mathcal{T}^{\perp}+B(\mathcal{T})$ es cerrado, o equivalentemente $R(G)=P_{\mathcal{T}}(B(\mathcal{T}))$ es cerrado. 
Recíprocamente, si $R\left(G_{B, \mathcal{S}}\right)$ es cerrado entonces $R\left(G_{B, \mathcal{S}}\right)=\mathcal{S} \ominus \mathcal{N}$ y $G_{B, \mathcal{S}}{ }^{\dagger} \in L(\mathcal{H})$. Consideremos el operador

$$
Q=P_{\mathcal{S}} G_{B, \mathcal{S}}^{\dagger} P_{\mathcal{S}} B \in L(\mathcal{H})
$$

Es fácil ver que $Q$ es una proyección oblicua tal que $B Q=Q^{*} B$ y $R(Q)=\mathcal{S} \ominus \mathcal{N}$. De hecho, $R(Q) \subseteq \mathcal{S}$ y, si $x \in \mathcal{N}$ entonces $Q x=0$. Por otra parte, si $x \in \mathcal{S} \ominus \mathcal{N}, Q x=P_{\mathcal{S}} P_{R\left(G_{B, \mathcal{S}}\right)} x=$ $P_{\mathcal{S}} x=x$. Luego, $R(Q)=\mathcal{S} \ominus \mathcal{N}$.

Notemos que $Q^{\prime}=Q+P_{\mathcal{N}}$ es una proyección oblicua con $R\left(Q^{\prime}\right)=\mathcal{S}$ y $B Q^{\prime}=\left(Q^{\prime}\right)^{*} B$ (porque $P_{\mathcal{N}} B=B P_{\mathcal{N}}=0$ ). Por lo tanto, $Q^{\prime} \in \mathcal{P}(B, \mathcal{S})$ i.e. $(B, \mathcal{S})$ is compatible.

Notemos que, si $\mathcal{M}=A^{1 / 2}(\mathcal{S})$ entonces $A^{-1 / 2}(\mathcal{M})=\mathcal{S}+N(A)$. Luego, el subespacio $\mathcal{M}$ es cerrado si y sólo si $\mathcal{S}+N(A)$ es cerrado.

Proposición 2.2.13. Sean $B \in C R(\mathcal{H})^{s}$ y $\mathcal{S}$ un subespacio cerrado de $\mathcal{H}$. Luego, $(B, \mathcal{S})$ es compatible si y sólo si $\mathcal{M}$ es cerrado y el par $(J, \mathcal{M})$ es compatible.

Demostración. Por la Proposición 2.2.1, la compatibilidad de $(B, \mathcal{S})$ implica que $\mathcal{M}$ es cerrado en $R\left(A^{1 / 2}\right)$. Entonces $\mathcal{M}$ es cerrado, pues $R\left(A^{1 / 2}\right)=R(B)$ es cerrado. Por otra parte, la Proposición 2.2.4 asegura que $R\left(A^{1 / 2}\right)=\mathcal{M}+J(\mathcal{M})^{\perp} \cap R\left(A^{1 / 2}\right)$ y, como

$$
J(\mathcal{M})^{\perp}=J(\mathcal{M})^{\perp} \cap R\left(A^{1 / 2}\right) \dot{+} N\left(A^{1 / 2}\right),
$$

tenemos que $\mathcal{H}=R\left(A^{1 / 2}\right)+N\left(A^{1 / 2}\right)=\mathcal{M}+J(\mathcal{M})^{\perp}$. La demostración de la recíproca es similar.

Corolario 2.2.14. Sea $B \in G L(\mathcal{H})^{s}$. Entonces, $(B, \mathcal{S})$ es compatible si y sólo si $(J, \mathcal{M})$ es compatible.

La proposición anterior prueba la equivalencia entre la compatibilidad de un par dado $(B, \mathcal{S})$ con la del par asociado $(J, \mathcal{M})$ - donde $J$ es la parte unitaria de la descomposición polar de $B$ y $\mathcal{M}=\overline{A^{1 / 2}(\mathcal{S})}$ - en el caso en que $B$ es un operador de rango cerrado. Es natural preguntarse sobre la posibilidad de extender este resultado al caso en que $B$ es un operador autoadjunto cualquiera. Sin embargo, el siguiente ejemplo muestra que existen pares $(B, \mathcal{S})$ no compatibles para los cuales el par asociado $(J, \mathcal{M})$ es compatible.

Ejemplo 2.2.15. Sean $\mathcal{S}$ y $\mathcal{T}$ dos subespacios cerrados de un espacio de Hilbert $\mathcal{H}$ (de dimensión infinita) tales que $c(\mathcal{S}, \mathcal{T})=1$. Consideremos el espacio de Hilbert $\mathcal{H}_{2}=\mathcal{H} \oplus \mathcal{H}$ y los operadores $A, J \in L\left(\mathcal{H}_{2}\right)$ definidos por

$$
A=\left(\begin{array}{cc}
P_{\mathcal{T} \perp} & 0 \\
0 & 0
\end{array}\right) \text { y } J=\left(\begin{array}{cc}
I & 0 \\
0 & -I
\end{array}\right)
$$

en la representación matricial inducida por $\mathcal{H} \oplus\{0\}$ como subespacio de $\mathcal{H}_{2}$. Observemos que $A \in L\left(\mathcal{H}_{2}\right)^{+}, R(A)=\mathcal{T}^{\perp}$ es cerrado y $J=J^{*}=J^{-1}$. Además, $J$ y $A$ conmutan, por lo que el operador $B=J A$ es autoadjunto.

Sea $\mathcal{M}=\overline{A^{1 / 2}(\mathcal{S})}=\overline{P_{\mathcal{T}^{\perp}}(\mathcal{S})}$. Como $\mathcal{M} \subseteq \mathcal{H} \oplus\{0\}$ es invariante por $J, J(\mathcal{M})^{\perp}=\mathcal{M}^{\perp} \oplus \mathcal{H}$ y, por lo tanto, $(J, \mathcal{M})$ es compatible. Por otra parte, $A^{1 / 2}(\mathcal{S})=P_{\mathcal{T} \perp}(\mathcal{S})$ no es cerrado (porque $c(\mathcal{S}, \mathcal{T})=1)$ y entonces $(B, \mathcal{S})$ no es compatible.

El próximo teorema presenta una caracterización completa de la compatibilidad de un par $(B, \mathcal{S})$ en términos del subespacio $\mathcal{M}$, para el caso particular en que $B \in C R(\mathcal{H})^{s}$. 
Teorema 2.2.16. Sea $B \in C R(\mathcal{H})^{s}$. Las siguientes condiciones son equivalentes:

1. $(B, \mathcal{S})$ es compatible.

2. $P_{\mathcal{M} / / J(\mathcal{M}) \perp} \in L(\mathcal{H})$.

3. $\mathcal{M}$ es cerrado y $c_{0}\left(\mathcal{M}, J(\mathcal{M})^{\perp}\right)<1$.

4. $R\left(G_{\mathcal{M}}\right)=\mathcal{M}$.

5. $\mathcal{M}$ es un espacio de Krein (con la métrica indefinida inducida por $J$ ).

Demostración. 1. $\Leftrightarrow$ 2.: Por la Proposición 2.2.13, $(B, \mathcal{S})$ es compatible si y sólo si $\mathcal{M}$ es cerrado y $\mathcal{H}=\mathcal{M}+J(\mathcal{M})^{\perp}$. Como $J$ es invertible, $\mathcal{M} \cap J(\mathcal{M})^{\perp}=\{0\}$ y, por lo tanto, $P_{\mathcal{M} / / J(\mathcal{M})^{\perp}} \in L(\mathcal{H})$.

2. $\Leftrightarrow$ 3.: Si $P_{\mathcal{M} / / J(\mathcal{M})^{\perp}} \in L(\mathcal{H})$ entonces $\mathcal{M}$ es cerrado, $\mathcal{M} \cap J(\mathcal{M})^{\perp}=\{0\}$ y $\mathcal{H}=\mathcal{M}+J(\mathcal{M})^{\perp}$. Por lo tanto, $c_{0}\left(\mathcal{M}, J(\mathcal{M})^{\perp}\right)=c\left(\mathcal{M}, J(\mathcal{M})^{\perp}\right)<1$. La recíproca es similar.

2. $\Leftrightarrow$ 4.: Recordemos que $N\left(G_{\mathcal{M}}\right)=\mathcal{M}^{\perp} \dot{+} \mathcal{M} \cap J(\mathcal{M})^{\perp}$. Si $(B, \mathcal{S})$ es compatible entonces, por la Proposición 2.2.12, $\mathcal{M} \cap J(\mathcal{M})^{\perp}=\{0\}$ y $R\left(G_{\mathcal{M}}\right)$ es cerrado. Luego, $R\left(G_{\mathcal{M}}\right)=N\left(G_{\mathcal{M}}\right)^{\perp}=\mathcal{M}$. Recíprocamente, si $R\left(G_{\mathcal{M}}\right)=\mathcal{M}$ entonces $N\left(G_{\mathcal{M}}\right)=\mathcal{M}^{\perp}$ i.e. $\mathcal{M} \cap J(\mathcal{M})^{\perp}=\{0\}$. Aplicando una vez más la Proposición 2.2.12, tenemos que $(J, \mathcal{M})$ es compatible i.e. $P_{\mathcal{M} / / J(\mathcal{M})^{\perp}} \in L(\mathcal{H})$.

Finalmente, la equivalencia $2 . \Leftrightarrow 5$. ya ha sido demostrada en el Teorema 1.2.37.

\subsection{Descomposición de proyecciones $B$-autoadjuntas}

Dentro del espacio vectorial real formado por los operadores $B$-autoadjuntos actuando sobre un espacio de Hilbert (complejo) $\mathcal{H}$, destacaremos a los operadores $B$-definidos.

Definición. Un operador $T \in L(\mathcal{H})$ es $B$-positivo si $\langle T x, x\rangle_{B} \geq 0$ para todo $x \in \mathcal{H}$, i.e. si $B T$ es un operador (semidefinido) positivo.

De la misma manera, diremos que un operador $T \in L(\mathcal{H})$ es $B$-negativo (resp. $B$-neutro) si $\langle T x, x\rangle_{B} \leq 0\left(\operatorname{resp} .\langle T x, x\rangle_{B}=0\right)$ para todo $x \in \mathcal{H}$.

Los operadores $B$-positivos forman un cono, ya que:

(a) si $T \in L(\mathcal{H})$ es $B$-positivo y $\alpha>0$ entonces $\alpha T$ es $B$-positivo;

(b) si $T_{1}, T_{2} \in L(\mathcal{H})$ son $B$-positivos entonces $T_{1}+T_{2}$ es $B$-positivo.

Luego, podemos definir un orden entre operadores $B$-autoadjuntos de la siguiente manera: dados operadores $B$-autoadjuntos $T, W \in L(\mathcal{H})$, diremos que $T \geq_{B} W$ si y sólo si $T-W$ es un operador $B$-positivo.

Observación 2.3.1. Sean $B \in L(\mathcal{H})^{s}$ y $Q \in \mathcal{Q}$ una proyección $B$-autoadjunta. Luego, $Q$ es $B$-positiva (resp. $B$-negativa, $B$-neutra) si y sólo si $R(Q)$ es un subespacio $B$-no negativo (resp. $B$-no positivo, $B$-neutro) de $\mathcal{H}$.

A continuación mostraremos que, si $B \in L(\mathcal{H})^{s}$ y $\mathcal{S}$ es un subespacio $B$-no degenerado de $\mathcal{H}$, entonces el par $(B, \mathcal{S})$ es compatible si y sólo si $\mathcal{S}$ puede descomponerse en suma directa de dos subespacios (cerrados) $B$-compatibles, uno $B$-no negativo y otro $B$-no positivo. 
Teorema 2.3.2 (Existencia de descomposición). Dados $\mathcal{S}$ un subespacio cerrado de $\mathcal{H}$ y $B \in$ $L(\mathcal{H})^{s}$, sea $\mathcal{N}=\mathcal{S} \cap N(B)$. Luego, $(B, \mathcal{S})$ es compatible si y sólo si existe una descomposición de $\mathcal{S} \ominus \mathcal{N}$ como $\mathcal{S} \ominus \mathcal{N}=\mathcal{S}_{+}+\mathcal{S}_{-}$, siendo $\mathcal{S}_{+}$un subespacio (cerrado) B-no negativo, $\mathcal{S}_{-}$un subespacio (cerrado) $B$-no positivo, $\left(B, \mathcal{S}_{ \pm}\right)$es compatible y $\mathcal{S}_{+} \perp_{B} \mathcal{S}_{-}$.

Demostración. Supongamos que $(B, \mathcal{S})$ es compatible. Luego, por el Lema 2.1.8, $(B, \mathcal{S} \ominus \mathcal{N})$ es compatible, $G_{B, \mathcal{S} \ominus \mathcal{N}}=G_{B, \mathcal{S}}$ y $G=\left.G_{B, \mathcal{S} \ominus \mathcal{N}}\right|_{\mathcal{S} \ominus \mathcal{N}}$ es inyectivo. Entonces, si $G=U|G|$ es la descomposición polar de $G, U$ es una simetría (i.e. $U=U^{*}=U^{-1}$ ) sobre $\mathcal{S} \ominus \mathcal{N}$. Sean $\mathcal{S}_{ \pm}$los autoespacios de $U$ correspondientes a $\lambda= \pm 1$ y recordemos que $\mathcal{S} \ominus \mathcal{N}=\mathcal{S}_{+} \oplus \mathcal{S}_{-}$.

Observemos además que, dado $x \in \mathcal{S}_{+}, x \neq 0$, se tiene que $\left.\langle B x, x\rangle=\langle G x, x\rangle=\langle|G| x, x\rangle\right\rangle$ 0 , es decir, $\mathcal{S}_{+}$es $B$-positivo. Análogamente, $\mathcal{S}_{-}$es $B$-negativo.

Dada $Q=P_{B, \mathcal{S} \ominus \mathcal{N}}$, consideremos $Q_{ \pm}=P_{\mathcal{S}_{ \pm}} Q$. Como $\mathcal{S}_{ \pm} \subseteq \mathcal{S} \ominus \mathcal{N}$, es fácil ver que $Q_{ \pm} \in \mathcal{Q}$ y $R\left(Q_{ \pm}\right)=\mathcal{S}_{ \pm}$. Además, $Q_{ \pm}$es $B$-autoadjunta, de hecho:

$$
\begin{aligned}
B Q_{ \pm} & =B P_{\mathcal{S}_{ \pm}} Q=B Q P_{\mathcal{S}_{ \pm}} Q=Q^{*} B P_{\mathcal{S}_{ \pm}} Q=Q^{*}\left(P_{\mathcal{S} \ominus \mathcal{N}}+P_{\mathcal{N}}\right) B P_{\mathcal{S}_{ \pm}} Q= \\
& =Q^{*} P_{\mathcal{S} \ominus \mathcal{N}} B P_{\mathcal{S}_{ \pm}} Q=Q^{*} G_{B, \mathcal{S} \ominus \mathcal{N}} P_{\mathcal{S}_{ \pm}} Q=Q^{*} P_{\mathcal{S}_{ \pm}} G_{B, \mathcal{S} \ominus \mathcal{N}} Q= \\
& =Q_{ \pm}^{*} B Q=Q_{ \pm}^{*} Q^{*} B=Q_{ \pm}^{*} B
\end{aligned}
$$

porque $G_{B, \mathcal{S} \ominus \mathcal{N}} P_{\mathcal{S}_{ \pm}}=P_{\mathcal{S}_{ \pm}} G_{B, \mathcal{S} \ominus \mathcal{N}}$ (pues $P_{\mathcal{S}_{ \pm}}$son proyectores espectrales del operador $G_{B, \mathcal{S} \ominus \mathcal{N}}$ ) y $Q Q_{ \pm}=Q_{ \pm}$. Por lo tanto, $\left(B, \mathcal{S}_{ \pm}\right)$es compatible.

Ya que $P_{\mathcal{S}_{+}} P_{\mathcal{S}_{-}}=P_{\mathcal{S}_{-}} P_{\mathcal{S}_{+}}=0, Q_{+} Q_{-}=P_{\mathcal{S}_{+}} Q P_{\mathcal{S}_{-}} Q=P_{\mathcal{S}_{+}} P_{\mathcal{S}_{-}} Q=0\left(\right.$ y también $Q_{-} Q_{+}=$ $0)$, es decir, $\mathcal{S}_{+} \perp_{B} \mathcal{S}_{-}$.

Recíprocamente, supongamos que $\mathcal{S} \ominus \mathcal{N}=\mathcal{S}_{+}+\mathcal{S}_{-}$y $\mathcal{S}_{ \pm}$satisfacen las condiciones mencionadas. Observemos que, por el Corolario 2.1.13, $\mathcal{P}\left(B, \mathcal{S}_{ \pm}\right)=\left\{Q_{ \pm}\right\}$y $N\left(Q_{ \pm}\right)=B\left(\mathcal{S}_{ \pm}\right)^{\perp}$ ya que $\mathcal{S}_{ \pm} \cap B\left(\mathcal{S}_{ \pm}\right)^{\perp}=\mathcal{S}_{ \pm} \cap N(B) \subseteq(\mathcal{S} \ominus \mathcal{N}) \cap N(B)=\{0\}$. Además, la $B$-ortogonalidad entre $\mathcal{S}_{+}$y $\mathcal{S}_{-}$ implica que $Q_{+} Q_{-}=Q_{-} Q_{+}=0$ pues $R\left(Q_{-}\right)=\mathcal{S}_{-} \subseteq \mathcal{S}_{+}^{\perp_{B}}=N\left(Q_{+}\right)$y $R\left(Q_{+}\right) \subseteq N\left(Q_{-}\right)$. Luego, si definimos $Q=Q_{+}+Q_{-}$, tenemos que $Q \in \mathcal{Q}, R(Q)=\mathcal{S}_{+}+\mathcal{S}_{-}=\mathcal{S} \ominus \mathcal{N}$ y $B Q=Q^{*} B$, i.e. $(B, \mathcal{S} \ominus \mathcal{N})$ es compatible. Luego, aplicando la Proposición 2.1.4, tenemos que $(B, \mathcal{S})$ es compatible.

En la Proposición 2.1.16 vimos que, si $(B, \mathcal{S})$ es compatible y $Q \in \mathcal{P}(B, \mathcal{S})$ entonces

$$
Q=P_{B, \mathcal{S} \ominus \mathcal{N}}+P_{\mathcal{N}} Q=P_{B, \mathcal{S} \ominus \mathcal{N}}+P_{\mathcal{N} / /(\mathcal{S} \ominus \mathcal{N}+N(Q))} .
$$

Mas aún, por el Teorema 2.3.2 sabemos que existen un subespacio $\mathcal{S}_{+}$(cerrado) $B$-no negativo y un subespacio $\mathcal{S}_{-}$(cerrado) $B$-no positivo tales que $\left(B, \mathcal{S}_{ \pm}\right)$es compatible y $\mathcal{S}_{+} \perp_{B} \mathcal{S}_{-}$. Luego, si $Q_{ \pm} \in \mathcal{P}\left(B, \mathcal{S}_{ \pm}\right)$, tenemos que

$$
Q=Q_{+}+Q_{-}+P_{\mathcal{N} / /(\mathcal{S} \ominus \mathcal{N}+N(Q))} .
$$

Además, $Q_{+}$es una proyección $B$-positiva, $Q_{-}$es una proyección $B$-negativa y $P_{\mathcal{N} / /(\mathcal{S} \ominus \mathcal{N}+N(Q))}$ es una proyección $B$-neutra.

Corolario 2.3.3. Sean $B \in L(\mathcal{H})^{s}$ y $\mathcal{S}$ un subespacio cerrado de $\mathcal{H}$ tales que $(B, \mathcal{S})$ es compatible. Entonces, cada $Q \in \mathcal{P}(B, \mathcal{S})$ admite una descomposición $Q=Q_{0}+Q_{+}+Q_{-}$tal que $Q_{0} \in \mathcal{Q}$ es $B$-neutra, $Q_{+} \in \mathcal{Q}$ es $B$-positiva y $Q_{-} \in \mathcal{Q}$ es $B$-negativa.

Cabe destacar que los subespacios $\mathcal{S}_{ \pm}$considerados en la demostración del Teorema 2.3.2 satisfacen condiciones adicionales a las pedidas en el mismo. Más precisamente, $\mathcal{S}_{+}$es un subespacio 
$B$-positivo, $\mathcal{S}_{-}$es un subespacio $B$-negativo, y éstos - además de ser $B$-ortogonales - son ortogonales con respecto al producto interno del espacio de Hilbert $\mathcal{H}$. El Teorema 2.3.4 muestra que, bajo estas hipótesis, hay una única descomposición posible.

Teorema 2.3.4 (Unicidad de la descomposición). Dados $\mathcal{S}$ un subespacio cerrado de $\mathcal{H}$ y $B \in$ $L(\mathcal{H})^{s}$, sea $\mathcal{N}=\mathcal{S} \cap N(B)$. Si $(B, \mathcal{S})$ es compatible entonces existe una única descomposición de $\mathcal{S} \ominus \mathcal{N}$ como $\mathcal{S} \ominus \mathcal{N}=\mathcal{S}_{+} \oplus \mathcal{S}_{-}$, siendo $\mathcal{S}_{+}$un subespacio (cerrado) B-positivo, $\mathcal{S}_{-}$un subespacio (cerrado) B-negativo, $\left(B, \mathcal{S}_{ \pm}\right)$es compatible y $\mathcal{S}_{+} \perp_{B} \mathcal{S}_{-}$.

Demostración. Sea $\mathcal{S} \ominus \mathcal{N}=\mathcal{V}_{+} \oplus \mathcal{V}_{-}$una descomposición con las condiciones mencionadas y consideremos $Q_{ \pm} \in \mathcal{P}\left(B, \mathcal{V}_{ \pm}\right)$. Luego, $Q_{ \pm} P_{\mathcal{V}_{ \pm}}=P_{\mathcal{V}_{ \pm}}$y $P_{\mathcal{V}_{+}} B P_{\mathcal{V}_{-}}=P_{\mathcal{V}_{-}} B P_{\mathcal{V}_{+}}=0$. De hecho,

$$
P_{\mathcal{V}_{+}} B P_{\mathcal{V}_{-}}=P \mathcal{V}_{+} B Q_{-} P_{\mathcal{V}_{-}}=P \mathcal{V}_{+} Q_{-}^{*} B P_{\mathcal{V}_{-}}=\left(Q_{-} P \mathcal{V}_{+}\right)^{*} B P \mathcal{V}_{-}=0
$$

porque $Q_{-} P_{\mathcal{V}_{+}}=Q_{-}\left(Q_{+} P_{\mathcal{V}_{+}}\right)=\left(Q_{-} Q_{+}\right) P_{\mathcal{V}_{+}}=0$. Entonces, $G_{B, \mathcal{S} \ominus \mathcal{N}}=G_{B, \mathcal{V}_{+}}+G_{B, \mathcal{V}_{-}}$. Como $\mathcal{V}_{ \pm}$son subespacios definidos, $G_{B, \mathcal{V}_{+}} \mid \mathcal{V}_{+} \in L\left(\mathcal{V}_{+}\right)^{+}$y $-G_{B, \mathcal{V}_{-}} \mid \mathcal{V}_{-} \in L\left(\mathcal{V}_{-}\right)^{+}$son inyectivos. Además, como $P_{\mathcal{V}_{+}} P_{\mathcal{V}_{-}}=P_{\mathcal{V}_{-}} P_{\mathcal{V}_{+}}=0$, se tiene que

$$
G_{B, \mathcal{S} \ominus \mathcal{N}}=G_{B, \mathcal{V}_{+}}+G_{B, \mathcal{V}_{-}}=\left(P_{\mathcal{V}_{+}}-P_{\mathcal{V}_{-}}\right)\left(G_{B, \mathcal{V}_{+}}-G_{B, \mathcal{V}_{-}}\right)
$$

Si definimos $A=G_{B, \mathcal{V}_{+}}-G_{B, \mathcal{V}_{-}}$, resulta que $A \in L(\mathcal{H})^{+}\left(\right.$notemos que $\left.A\right|_{\mathcal{V}_{+} \oplus \mathcal{V}_{-}} \in L\left(\mathcal{V}_{+} \oplus \mathcal{V}_{-}\right)^{+}$ también es inyectivo) y, si $U=P_{\mathcal{V}_{+}}-P_{\mathcal{V}_{-}}$, entonces $U=U^{*}$ y $U^{2}=P_{\mathcal{S} \ominus \mathcal{N}}$. Entonces,

$$
G=\left.G_{B, \mathcal{S} \ominus \mathcal{N}}\right|_{\mathcal{S} \ominus \mathcal{N}}=\left.\left.U\right|_{\mathcal{S} \ominus \mathcal{N}} A\right|_{\mathcal{S} \ominus \mathcal{N}}
$$

siendo $\left.A\right|_{\mathcal{S} \ominus \mathcal{N}} \in L(\mathcal{S} \ominus \mathcal{N})^{+}$y $\left.U\right|_{\mathcal{S} \ominus \mathcal{N}} \in L(\mathcal{S} \ominus \mathcal{N})$ una simetría. Luego, por la unicidad de la descomposición polar, $\left.U\right|_{\mathcal{S} \ominus \mathcal{N}}$ es la parte unitaria de $G$ y $\mathcal{V}_{ \pm}$es el autoespacio de $\left.U\right|_{\mathcal{S} \ominus \mathcal{N}}$ correspondiente a $\lambda= \pm 1$.

Utilizando el teorema anterior podemos asegurar que, entre las posibles descomposiciones provistas por el Corolario 2.3.3, existe una única para la cual los rangos de las proyecciones son dos a dos ortogonales (con respecto al producto interno de $\mathcal{H}$ ) y además los rangos de las proyecciones definidas son estrictamente definidos.

Corolario 2.3.5. Dados $B \in L(\mathcal{H})^{s}$ y un subespacio cerrado $\mathcal{S}$ de $\mathcal{H}$, sean $Q \in \mathcal{P}(B, \mathcal{S})$ y $Q_{0}$, $Q_{+} y Q_{-}$proyecciones tales que $Q=Q_{0}+Q_{+}+Q_{-}$, $Q_{0}$ es $B$-neutra, $Q_{+}$es B-positiva y $Q_{-}$ es $B$-negativa. Supongamos además que sus rangos satisfacen las siguientes condiciones:

(i) son dos a dos B-ortogonales;

(ii) son dos a dos ortogonales (con respecto al producto interno de $\mathcal{H}$ );

(iii) $R\left(Q_{+}\right)$es B-positivo y $R\left(Q_{-}\right)$es $B$-negativo.

Entonces,

$$
Q_{0}=P_{\mathcal{N} / / N(Q) \dot{+} \mathcal{S} \ominus \mathcal{N}}, \quad Q_{+}=P_{B, \mathcal{S}_{+}} \quad y \quad Q_{-}=P_{B, \mathcal{S}_{-}},
$$

siendo $\mathcal{S} \ominus \mathcal{N}=\mathcal{S}_{+} \oplus \mathcal{S}_{-}$la descomposición dada en el Teorema 2.3.4. 
Demostración. Sean $\mathcal{V}_{0}=R\left(Q_{0}\right), \mathcal{V}_{+}=R\left(Q_{+}\right)$y $\mathcal{V}_{-}=R\left(Q_{-}\right)$. En primer lugar, notemos que, $\mathcal{S} \subseteq \mathcal{V}_{0} \dot{+} \mathcal{V}_{+} \dot{+} \mathcal{V}_{-}=\mathcal{V}_{0} \oplus \mathcal{V}_{+} \oplus \mathcal{V}_{-}$, donde la última igualdad es consecuencia del item (ii). Recíprocamente, si $x=v_{0}+v_{+}+v_{-} \in \mathcal{V}_{0} \oplus \mathcal{V}_{+} \oplus \mathcal{V}_{-}$, utilizando el item $(i)$ se tiene que

$$
\begin{aligned}
Q x=\left(Q_{0}+Q_{+}+Q_{-}\right) x & =\left(Q_{0}+Q_{+}+Q_{-}\right)\left(v_{0}+v_{+}+v_{-}\right)=\ldots= \\
& =Q_{0} v_{0}+Q_{+} v_{+}+Q_{-} v_{-}=v_{0}+v_{+}+v_{-}=x \in \mathcal{S} .
\end{aligned}
$$

Además, $\mathcal{V}_{1} \oplus \mathcal{V}_{2}=\mathcal{S} \ominus \mathcal{N}$ : como $B Q_{0}=0$, se tiene que $\mathcal{V}_{0} \subseteq \mathcal{S} \cap N(B)=\mathcal{N}$. Luego, considerando el complemento ortogonal en $\mathcal{S}, \mathcal{S} \ominus \mathcal{N} \subseteq \mathcal{V}_{+} \oplus \mathcal{V}_{-}$y entonces $\mathcal{V}_{+} \oplus \mathcal{V}_{-}=(\mathcal{S} \ominus \mathcal{N}) \oplus \mathcal{U}$ y $\mathcal{U} \subseteq \mathcal{N}$. Pero $\mathcal{U}=\{0\}$ pues, si $u \in \mathcal{U}, u \neq 0$ entonces $u=v_{+}+v_{-}$con $v_{+} \in \mathcal{V}_{+}, v_{-} \in \mathcal{V}_{-}, v_{+} \neq 0\left(\right.$ or $\left.v_{-} \neq 0\right)$ y $B u=0$. Por lo tanto, $B v_{+}=-B v_{-}$y, por el item (iii), $0\left\langle\left\langle B v_{+}, v_{+}\right\rangle=-\left\langle B v_{-}, v_{+}\right\rangle=\right.$ $-\left\langle v_{-}, B v_{+}\right\rangle=\left\langle B v_{-}, v_{-}\right\rangle\left\langle 0\right.$, lo que resulta absurdo. Entonces, $\mathcal{V}_{1} \oplus \mathcal{V}_{2}=\mathcal{S} \ominus \mathcal{N}$ y la unicidad de la descomposición es una consecuencia del Teorema 2.3.4.

\subsubsection{Proyecciones $B$-contractivas y $B$-expansivas}

Sea $Q$ una proyección actuando sobre un espacio de Hilbert $\mathcal{H}$. De la teoría clásica de operadores sobre espacios de Hilbert sabemos que

$$
Q^{*}=Q \quad \Leftrightarrow \quad\|Q\| \leq 1
$$

es decir, en la variedad formada por las proyecciones que actúan sobre $\mathcal{H}$, las proyecciones ortogonales están caracterizadas por la contractividad. Esto nos motiva a preguntarnos, fijado $B \in L(\mathcal{H})^{s}$, en qué casos puede encontrarse un resultado análogo para proyecciones $B$ autoadjuntas. Veremos que, en el caso indefinido, este problema no tiene una respuesta inmediata como la que mencionamos anteriormente para espacios de Hilbert.

Definición. Dado $B \in L(\mathcal{H})^{s}$ consideremos el espacio con métrica indefinida $\left(\mathcal{H},\langle,\rangle_{B}\right)$. Un operador $T \in L(\mathcal{H})$ es una $B$-contracción si

$$
\langle T x, T x\rangle_{B} \leq\langle x, x\rangle_{B} \quad \text { para todo } x \in \mathcal{H},
$$

o equivalentemente, $T^{*} B T \leq B$.

En primer lugar, recordemos que ocurre si consideramos una perturbación positiva $A \in$ $L(\mathcal{H})^{+}$. Este es un resultado conocido que puede citarse, por ejemplo, de [19, Lema 3.2].

Proposición 2.3.6. Sean $A \in L(\mathcal{H})^{+}$y $Q \in \mathcal{Q}$. Luego, $Q$ es A-autoadjunta si y sólo si $Q$ es A-contractiva.

Demostración. En primer lugar, supongamos que $0 \leq Q^{*} A Q \leq A$. Como $\left(Q^{*} A^{1 / 2}\right)\left(Q^{*} A^{1 / 2}\right)^{*}=$ $Q^{*} A Q \leq A=A^{1 / 2}\left(A^{1 / 2}\right)^{*}$, por el Teorema de Douglas, sabemos que existe una solución reducida $E \in L(\mathcal{H})$ de la ecuación $A^{1 / 2} X=Q^{*} A^{1 / 2}$ y esta cumple que $\|E\| \leq 1$. Además, por el Corolario 1.1.3, tenemos que $E \in \mathcal{Q}$ y la Ec. (2.3.1) asegura que $E \in \mathcal{P}$. Luego, $Q^{*} A=A^{1 / 2} E A^{1 / 2}$ es un un operador autoadjunto, i.e. $Q^{*} A=A Q$.

Recíprocamente, supongamos que $Q$ es $A$-autoadjunta. Notemos que $A Q=(A Q) Q=$ $\left(Q^{*} A\right) Q=Q^{*} A Q \in L(\mathcal{H})^{+}$y, si llamamos $E=I-Q$, entonces también $A E=E^{*} A E \in L(\mathcal{H})^{+}$. Por lo tanto,

$$
A=A(Q+E)=Q^{*} A Q+E^{*} A E \geq Q^{*} A Q .
$$


Para un operador autoadjunto $B \in L(\mathcal{H})^{s}$, S. Hassi y K. Nordström [38, Proposición 5] probaron el siguiente resultado, que caracteriza aquellas proyecciones $B$-autoadjuntas que son $B$-contractivas.

Proposición 2.3.7. Sea $B \in L(\mathcal{H})^{\text {s. }}$. Si $Q \in \mathcal{Q}$ entonces, $Q$ es una $B$-contracción si y sólo si $Q$ es $B$-autoadjunta y $N(Q)$ es un subespacio $B$-no negativo.

Demostración. Supongamos que $Q$ es una $B$-contracción, es decir, $B-Q^{*} B Q \in L(\mathcal{H})^{+}$. Luego, si $C=\left(B-Q^{*} B Q\right)^{1 / 2} Q \in L(\mathcal{H})$, tenemos que $C^{*} C=Q^{*}\left(B-Q^{*} B Q\right) Q=0$. Entonces, $C=0$ y, multiplicando por $\left(B-Q^{*} B Q\right)^{1 / 2}$, vemos que $\left(B-Q^{*} B Q\right) Q=0$. Por lo tanto, $B Q=Q^{*} B Q \in L(\mathcal{H})^{s}$. Además, como consecuencia de (2.3.2) tenemos que $N(Q)$ es $B$-no negativo.

Recíprocamente, si $Q$ es $B$-autoadjunta entonces $(I-Q)^{*} B(I-Q)=B-Q^{*} B Q$. Luego, si $N(Q)$ es $B$-no negativo tenemos que, para todo $x \in \mathcal{H}$,

$$
\left\langle\left(B-Q^{*} B Q\right) x, x\right\rangle=\left\langle(I-Q)^{*} B(I-Q) x, x\right\rangle=\langle B(I-Q) x,(I-Q) x\rangle=0,
$$

es decir, $Q$ es $B$-contractiva.

Observación 2.3.8. Sean $B \in L(\mathcal{H})^{s}$ y $Q \in \mathcal{Q}$. Entonces,

1. $Q$ es $B$-contractiva si y sólo si $I-Q$ es $B$-positiva. De hecho, si $Q$ es $B$-contractiva entonces $Q$ es $B$-autoadjunta i.e. $B Q=Q^{*} B$. Luego, $B(I-Q)=B-B Q=B-Q^{*} B Q \geq 0$. Recíprocamente, si $I-Q$ es $B$-positiva entonces $I-Q$ y $Q$ son proyecciones $B$-autoadjuntas. Además, $B \geq B Q=Q^{*} B Q$ i.e. $Q$ es $B$-contractiva.

2. Un operador $T \in L(\mathcal{H})$ es una $B$-expansión si $\langle T x, T x\rangle_{B} \geq\langle x, x\rangle_{B}$ (i.e. $T^{*} B T \geq B$ ) y $T$ es una $B$-isometría si $\langle T x, T x\rangle_{B}=\langle x, x\rangle_{B}$ (i.e. $T^{*} B T=B$ ). Siguiendo un argumento análogo al del item anterior se puede probar que $Q \in \mathcal{Q}$ es $B$-expansiva (respectivamente $B$-isométrica) si y sólo si $I-Q$ es $B$-negativa (respectivamente $B$-neutra).

Finalmente, mostraremos que las proyecciones $B$-autoadjuntas pueden factorizarse en términos de proyecciones $B$-contractivas, $B$-expansivas y $B$-isométricas.

Teorema 2.3.9. Toda proyección B-autoadjunta $Q \in L(\mathcal{H})$ admite una factorización $Q=$ $Q_{0} Q_{1} Q_{2}$, siendo $Q_{0}, Q_{1}$ y $Q_{2}$ proyecciones en $L(\mathcal{H})$ que conmutan entre sí y tales que $Q_{0}$ es $B$-isométrica, $Q_{1}$ es B-contractiva y $Q_{2}$ es $B$-expansiva.

Demostración. Si $Q$ es $B$-autoadjunta entonces $I-Q \in \mathcal{P}(B, N(Q))$ y, por el Corolario 2.3.3,

$$
I-Q=E_{0}+E_{+}+E_{-},
$$

con $E_{0}$ una proyección $B$-neutra, $E_{+}$una proyección $B$-positiva y $E_{-}$una proyección $B$-negativa. Además, estas proyecciones satisfacen $E_{+} E_{-}=E_{-} E_{+}=E_{+} E_{0}=E_{0} E_{+}=E_{-} E_{0}=E_{0} E_{-}=0$. Si llamamos $Q_{0}=I-E_{0}, Q_{1}=I-E_{+}$y $Q_{2}=I-E_{-}$tenemos que estas proyecciones conmutan entre sí y

$$
Q=I-\left(E_{0}+E_{+}+E_{-}\right)=\left(I-E_{0}\right)\left(I-E_{+}\right)\left(I-E_{-}\right)=Q_{0} Q_{1} Q_{2} .
$$

Notemos que, por la Observación $2.3 .8, Q_{0}$ es $B$-isométrica, $Q_{1}$ es $B$-contractiva y $Q_{2}$ es $B$ expansiva. 
Observación 2.3.10. El Corolario 2.3.5 también nos asegura la unicidad de esta factorización bajo las siguientes hipótesis sobre los núcleos de las proyecciones $Q_{i}$ :

(i) son dos a dos $B$-ortogonales;

(ii) son dos a dos ortogonales (con respecto al producto interno de $\mathcal{H}$ );

(iii) $N\left(Q_{1}\right)$ es $B$-positivo y $N\left(Q_{2}\right)$ es $B$-negativo.

En [38, Teorema 2], S. Hassi y K. Nordström probaron un caso particular del Teorema 2.3.9.

Corolario 2.3.11 (Hassi \& Nordström). Si $B \in G L(\mathcal{H})^{s}$, toda proyección B-autoadjunta $Q$ puede representarse como el producto de dos proyecciones $Q_{1}$ y $Q_{2}$ que conmutan entre sí y tales que $Q_{1}$ es $B$-contractiva y $Q_{2}$ es $B$-expansiva.

\subsubsection{El fibrado de descomposiciones canónicas de un espacio de Krein}

Para finalizar esta sección daremos una descripción de las decomposiciones canónicas de un espacio de Krein. Dado un espacio de $\operatorname{Krein}(\mathcal{H},[]$,$) con simetría fundamental J$, una descomposición canónica de $\mathcal{H}$ es una descomposición de $\mathcal{H}$ como una suma directa

$$
\mathcal{H}=\mathcal{S} \dot{+} \mathcal{S}^{[\perp]},
$$

siendo $\mathcal{S}$ y $\mathcal{S}^{[\perp]}$ subespacios cerrados de $\mathcal{H}$ tales que $\mathcal{S}$ es $J$-positivo y $\mathcal{S}^{[\perp]}$ es $J$-negativo. Observemos que $\mathcal{H}=\mathcal{S}+\mathcal{S}^{[\perp]}$ es una descomposición canónica de $\mathcal{H}$ si y sólo si $P_{\mathcal{S} / / \mathcal{S}^{[\perp]}}$ es $B$-expansiva y $I-P_{\mathcal{S} / / \mathcal{S}}[\perp]$ es $B$-contractiva (para más detalles, consultar la sección 1.2.2 de los preliminares).

Luego, cada descomposición canónica define una reflexión $K_{\mathcal{S}}$ (i.e. un operador acotado invertible que coincide con su inversa), por medio de $K_{\mathcal{S}}=2 P_{\mathcal{S} / / \mathcal{S}}{ }^{[\perp]}-I$.

Lema 2.3.12. Dado $B \in L(\mathcal{H})^{s}$, sea $Q \in \mathcal{Q}$ tal que $R(Q)$ es $B$-no negativo y $N(Q)$ es $B$-no positivo. Entonces, $Q$ es $B$-autoadjunta si y sólo si la reflexión $2 Q-I$ es $B$-positiva.

Demostración. Supongamos que $Q$ es una proyección $B$-autoadjunta. Luego, si $K=2 Q-I$,

$$
\langle K x, x\rangle_{B}=\langle Q x, x\rangle_{B}-\langle(I-Q) x, x\rangle_{B}=\langle Q x, Q x\rangle_{B}-\langle(I-Q) x,(I-Q) x\rangle_{B} \geq 0,
$$

porque $R(Q)$ es $B$-no negativo y $N(Q)$ es $B$-no positivo. Por lo tanto, $K$ es $B$-positiva. Recíprocamente, si $K$ es $B$-positiva entonces $K$ es $B$-autoadjunta y también $Q=\frac{I+K}{2}$ es $B$-autoadjunta.

Consideremos el conjunto de reflexiones $\mathcal{K}=\left\{K \in G L(\mathcal{H}): K^{-1}=K\right\}$ y el conjunto de simetrías $\mathcal{J}=\left\{J \in G L(\mathcal{H})^{s}: J^{-1}=J\right\} \subset \mathcal{K}$. Si $K \in \mathcal{K}$ y $K=J_{K}|K|$ es la descomposición polar de $K$ entonces $J_{K} \in \mathcal{J}$ (ver [25, Proposición 3.1]). Entonces, la aplicación

$$
\pi: \mathcal{K} \rightarrow \mathcal{J}, \quad \pi(K)=J_{K},
$$

está bien definida y es continua. Además, el conjunto de decomposiciones canónicas de $\mathcal{H}$ pueden parametrizarse mediante las reflexiones en la fibra $\pi^{-1}(\{J\})$.

El contenido del siguiente resultado puede leerse como una reformulación de las proposiciones 3.1 y 3.2 de [25], sin embargo las técnicas utilizadas en este caso son distintas. 
Proposición 2.3.13. Sea $\mathcal{H}$ un espacio de Krein con simetría fundamental $J$. Entonces, $\mathcal{H}=$ $\mathcal{S} \dot{+} \mathcal{S}^{[\perp]}$ es una descomposición canónica de $\mathcal{H}$ si y sólo si $K_{\mathcal{S}} \in \pi^{-1}(\{J\})$.

Demostración. Supongamos que $\mathcal{H}=\mathcal{S}+\mathcal{S}^{[\perp]}$, o equivalentemente, $P_{\mathcal{S} / / \mathcal{S}[\perp]}$ es $J$-autoadjunta, $\mathcal{S}$ es $J$-positivo y $\mathcal{S}^{[\perp]}$ es $J$-negativo. Por el Lema $2.3 .12, K_{\mathcal{S}}$ es un operador $J$-positivo i.e. $A:=J K \in L(\mathcal{H})^{+}$. Luego, $K=J A$ y $A^{*} A=K^{*} J^{2} K=K^{*} K$, es decir, $A=|K|$ y $J$ es la parte unitaria de la descomposición polar de $K$. Por lo tanto, $K_{\mathcal{S}} \in \pi^{-1}(\{J\})$.

Recíprocamente, si $K \in \pi^{-1}(\{J\})$ entonces $K$ es $J$-positivo. Por el Lema 2.3.12, la proyección $Q=\frac{I+K}{2}$ es $J$-autoadjunta y es fácil probar que $\mathcal{S}:=R(Q)$ es $J$-positivo y $\mathcal{S}^{[\perp]}=N(Q)$ es $J$-negativo. Por lo tanto, $\mathcal{H}=\mathcal{S}+\mathcal{S}^{[\perp]}$ es una descomposición canónica de $\mathcal{H}$.

\subsection{Fórmulas para $P_{B, \mathcal{S}}$}

El objetivo de esta sección es obtener fórmulas para ciertas proyecciones $B$-autoadjuntas en términos de la descomposición polar de $B$ y de proyecciones $J$-autoadjuntas, en cierto espacio de Krein con simetría fundamental $J$. Comenzaremos estudiando el caso de una perturbación autoadjunta inversible y posteriormente intentaremos generalizar estos resultados a un contexto más amplio.

Sea $B \in G L(\mathcal{H})^{s}$ y consideremos su descomposición polar $B=J A$, con $A \in G L(\mathcal{H})^{+}$y $J=J^{*}=J^{-1}$. Luego,

$$
\langle x, y\rangle_{B}=\langle B x, y\rangle=\langle A J x, y\rangle=\langle J x, y\rangle_{A}, \quad \text { para todo } x, y \in \mathcal{H} .
$$

Como $A \in G L(\mathcal{H})^{+}$, la forma sesquilineal $\langle,\rangle_{A}$ es un producto interno en $\mathcal{H}$ equivalente al producto interno original $\langle$,$\rangle . Además, como J$ conmuta con $A$, tenemos que $J$ también es una simetría con respecto al producto interno $\langle,\rangle_{A} \mathrm{y}$, por lo tanto, $\left(\mathcal{H},\langle,\rangle_{A}\right)$ también es un espacio de Krein con simetría fundamental $J$.

Recordemos que, en este caso, el Corolario 2.2.14 asegura que la compatibilidad del par $(B, \mathcal{S})$ es equivalente a la existencia de una (única) proyección $J$-ortogonal con rango $\mathcal{M}=A^{1 / 2}(\mathcal{S})$, en el espacio de $\operatorname{Krein}\left(\mathcal{H},\langle,\rangle_{A}\right)$.

Proposición 2.4.1. Sean $B \in G L(\mathcal{H})^{s}$ y $\mathcal{S}$ un subespacio cerrado de $\mathcal{H}$. Si $(B, \mathcal{S})$ es compatible, entonces

$$
P_{B, \mathcal{S}}=A^{-1 / 2} P_{\mathcal{M} / / J(\mathcal{M})^{\perp}} A^{1 / 2} .
$$

Demostración. Si $(B, \mathcal{S})$ es compatible entonces, por el Teorema 2.2.16, $P_{\mathcal{M} / / J(\mathcal{M})^{\perp}} \in L(\mathcal{H})$. Consideremos el operador $Q=A^{-1 / 2} P_{\mathcal{M} / / J(\mathcal{M})^{\perp}} A^{1 / 2} \in L(\mathcal{H})$. Notemos que $Q$ es una proyección con $R(Q)=A^{-1 / 2}(\mathcal{M})=\mathcal{S}$ y además $B Q=J A^{1 / 2} P_{\mathcal{M} / / J(\mathcal{M})^{\perp}} A^{1 / 2}=Q^{*} B$ (recordemos que $J$ y $A^{1 / 2}$ conmutan y que $P_{\mathcal{M} / / J(\mathcal{M})^{\perp}}$ es $J$-autoadjunta). Luego, como $\mathcal{P}(B, \mathcal{S})$ posee un solo elemento (ver Corolario 2.1.13), tenemos que $Q=P_{B, \mathcal{S}}$.

Consideremos ahora un operador $B \in L(\mathcal{H})^{s}$ no necesariamente inversible, con descomposición polar $B=J A$, siendo $A \in L(\mathcal{H})^{+}$y $J=J^{*}=J^{-1}$. Si $\mathcal{S}$ es un subespacio cerrado de $\mathcal{H}$, sea $P=P_{\mathcal{S}}$ la proyección ortogonal sobre $\mathcal{S}$ y definamos el subespacio cerrado $\mathcal{M}=\overline{A^{1 / 2}(\mathcal{S})}$.

Recordemos que, por el Corolario 2.2.6, si $(B, \mathcal{S})$ es compatible entonces $\mathcal{M}$ es un subespacio $J$-no degenerado del espacio de Krein $\mathcal{H}$ con simetría fundamental $J$. Por lo tanto, en 
general, la proyección $P_{\mathcal{M} / / J(\mathcal{M})^{\perp}}$ tiene dominio denso (el subespacio $\mathcal{M} \dot{+} J(\mathcal{M})^{\perp}$ ) pero puede resultar un operador no acotado. Sin embargo, la siguiente proposición muestra que el producto $P_{\mathcal{M} / / J(\mathcal{M}) \perp} A^{1 / 2}$ permanece acotado.

Proposición 2.4.2. Sean $B \in L(\mathcal{H})^{s}$ y $\mathcal{S}$ un subespacio cerrado de $\mathcal{H}$. Si $(B, \mathcal{S})$ es compatible entonces el operador $T=P_{\mathcal{M} / / J(\mathcal{M})^{\perp}} A^{1 / 2}$ resulta bien definido y acotado.

Demostración. Si el par $(B, \mathcal{S})$ es compatible entonces, por el Corolario 2.2.5, sabemos que

$$
R\left(A^{1 / 2}\right)=\mathcal{M} \cap R\left(A^{1 / 2}\right) \dot{+} J(\mathcal{M})^{\perp} \cap R\left(A^{1 / 2}\right) \subseteq \mathcal{M}+J(\mathcal{M})^{\perp} .
$$

Luego, $T$ está bien definido pues $\operatorname{Dom}\left(P_{\mathcal{M} / / J(\mathcal{M})^{\perp}}\right)=\mathcal{M}+J(\mathcal{M})^{\perp}$. Dada $Q \in \mathcal{P}(B, \mathcal{S})$, para todo $x \in \mathcal{H}$ tenemos que

$$
T x=T Q x+T(I-Q) x .
$$

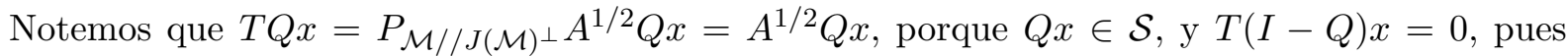
$A^{1 / 2}(I-Q) x \in A^{1 / 2}\left(B^{-1}\left(\mathcal{S}^{\perp}\right)\right)=J\left(A^{1 / 2}(\mathcal{S})\right)^{\perp} \cap R\left(A^{1 / 2}\right) \subseteq J(\mathcal{M})^{\perp}$. Por lo tanto,

$$
P_{\mathcal{M} / / J(\mathcal{M})^{\perp}} A^{1 / 2}=A^{1 / 2} Q \in L(\mathcal{H}) .
$$

En [20, Proposición 2.14], G. Corach et al. probaron el siguiente resultado para perturbaciones positivas de un producto interno:

Proposición 2.4.3. Si $A \in L(\mathcal{H})^{+}$y $\mathcal{S}$ es un subespacio cerrado de $\mathcal{H}$, entonces $(A, \mathcal{S})$ es compatible si y sólo si

$$
R\left(P_{\mathcal{M}} A^{1 / 2}\right) \subseteq R\left(A^{1 / 2} P\right) .
$$

Los próximos corolarios son generalizaciones del mismo.

Corolario 2.4.4. Dados $B \in L(\mathcal{H})^{s}$ y un subespacio cerrado $\mathcal{S}$ de $\mathcal{H}$, el par $(B, \mathcal{S})$ es compatible si y sólo si $R\left(A^{1 / 2}\right) \subseteq \mathcal{M} \dot{+} J(\mathcal{M})^{\perp} y$

$$
R\left(P_{\mathcal{M} / / J(\mathcal{M})^{\perp}} A^{1 / 2}\right) \subseteq R\left(A^{1 / 2} P\right) .
$$

Demostración. Si $(B, \mathcal{S})$ es compatible entonces, por el Corolario 2.2.5, sabemos que $R\left(A^{1 / 2}\right) \subseteq$

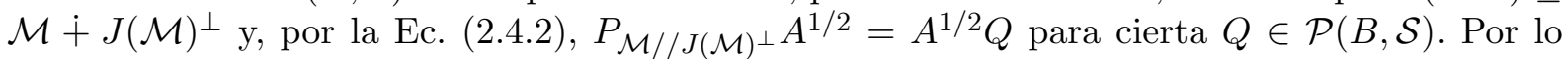
tanto, $R\left(P_{\mathcal{M} / / J(\mathcal{M}) \perp} A^{1 / 2}\right)=R\left(A^{1 / 2} Q\right)=R\left(A^{1 / 2} P\right)$.

Recíprocamente, supongamos que $R\left(A^{1 / 2}\right) \subseteq \mathcal{M} \dot{+} J(\mathcal{M})^{\perp}$ y $R\left(P_{\mathcal{M} / / J(\mathcal{M})^{\perp}} A^{1 / 2}\right) \subseteq R\left(A^{1 / 2} P\right)$. Dado $y \in R\left(A^{1 / 2}\right)$, consideremos los vectores $y_{1}=P_{\mathcal{M} / / J(\mathcal{M}) \perp} y$ e $y_{2}=\left(I-P_{\mathcal{M} / / J(\mathcal{M}) \perp}\right) y$. Notemos que $y_{1} \in R\left(P_{\mathcal{M} / / J(\mathcal{M})^{\perp}} A^{1 / 2}\right) \subseteq R\left(A^{1 / 2} P\right)=A^{1 / 2}(\mathcal{S})$. Luego, es fácil ver que $y_{2}=y-y_{1} \in$ $R\left(A^{1 / 2}\right)$ y además $y_{2} \in N\left(P_{\mathcal{M} / / J(\mathcal{M})^{\perp}}\right)=J(\mathcal{M})^{\perp}$. Entonces, $y_{2} \in J\left(A^{1 / 2}(\mathcal{S})\right)^{\perp} \cap R\left(A^{1 / 2}\right)$ y la descomposición $R\left(A^{1 / 2}\right)=A^{1 / 2}(\mathcal{S}) \dot{+} J\left(A^{1 / 2}(\mathcal{S})\right)^{\perp} \cap R\left(A^{1 / 2}\right)$ queda probada. Finalmente, como consecuencia de la Proposición 2.2.4, tenemos que el par $(B, \mathcal{S})$ es compatible.

Corolario 2.4.5. Sea $B=J A$ la descomposición polar de $B \in L(\mathcal{H})^{s}$, con $A \in L(\mathcal{H})^{+}$y $J=J^{*}=J^{-1}$. Dado un subespacio cerrado $\mathcal{S}$ de $\mathcal{H}$, consideremos el subespacio $\mathcal{M}=\overline{A^{1 / 2}(\mathcal{S})}$. Entonces, $(B, \mathcal{S})$ es compatible si y sólo si se cumplen las siguientes condiciones: 

1. $P_{\mathcal{M} / / J(\mathcal{M})^{\perp}} A^{1 / 2} \in L(\mathcal{H})$,
2. $A^{1 / 2}(\mathcal{S})$ es cerrado en $R\left(A^{1 / 2}\right)$,
3. $R\left(P_{\mathcal{M} / / J(\mathcal{M})^{\perp}} A^{1 / 2}\right) \subseteq R\left(A^{1 / 2}\right)$.

Demostración. Si $(B, \mathcal{S})$ es compatible, $P_{\mathcal{M} / / J(\mathcal{M})^{\perp}} A^{1 / 2} \in L(\mathcal{H})$ como consecuencia de la Proposición 2.4.2. Además, por el Corolario 2.2.5, sabemos que $A^{1 / 2}(\mathcal{S})$ es cerrado en $R\left(A^{1 / 2}\right)$ y

$$
R\left(A^{1 / 2}\right)=A^{1 / 2}(\mathcal{S})+J(\mathcal{M})^{\perp} \cap R\left(A^{1 / 2}\right) .
$$

Por lo tanto, $R\left(P_{\mathcal{M} / / J(\mathcal{M})^{\perp}} A^{1 / 2}\right)=A^{1 / 2}(\mathcal{S}) \subseteq R\left(A^{1 / 2}\right)$.

Recíprocamente, supongamos que se cumplen las condiciones 1 , 2 y 3 . Si $P_{\mathcal{M} / / J(\mathcal{M})} A^{1 / 2} \in$ $L(\mathcal{H})$ entonces $\left(I-P_{\mathcal{M} / / J(\mathcal{M})^{\perp}}\right) A^{1 / 2}$ también está acotado y, para todo $x \in \mathcal{H}$,

$$
A^{1 / 2} x=P_{\mathcal{M} / / J(\mathcal{M})^{\perp}} A^{1 / 2} x+\left(I-P_{\mathcal{M} / / J(\mathcal{M})^{\perp}}\right) A^{1 / 2} x .
$$

Observemos que $P_{\mathcal{M} / / J(\mathcal{M})^{\perp}} A^{1 / 2} x \in R\left(P_{\mathcal{M} / / J(\mathcal{M})^{\perp}} A^{1 / 2}\right) \subseteq \mathcal{M} \cap R\left(A^{1 / 2}\right)$, y en consecuencia

$$
\left(I-P_{\mathcal{M} / / J(\mathcal{M})^{\perp}}\right) A^{1 / 2} x \in J(\mathcal{M})^{\perp} \cap R\left(A^{1 / 2}\right) .
$$

Luego, $R\left(A^{1 / 2}\right)=\mathcal{M} \cap R\left(A^{1 / 2}\right)+J(\mathcal{M})^{\perp} \cap R\left(A^{1 / 2}\right)$ y, por el Corolario 2.2.5, el par $(B, \mathcal{S})$ es compatible.

Consideremos la ecuación $\left(A^{1 / 2} P\right) X=P_{\mathcal{M} / / J(\mathcal{M})^{\perp}} A^{1 / 2}$ y recordemos que $D$ es su solución reducida si y sólo si $R(D) \subseteq \overline{R\left(\left(A^{1 / 2} P\right)^{*}\right)} \subseteq R(P)=\mathcal{S}$. Entonces,

$$
A^{1 / 2} D=P_{\mathcal{M} / / J(\mathcal{M})^{\perp}} A^{1 / 2} .
$$

Además, si $A^{1 / 2}$ es inyectivo, tenemos que $A^{-1 / 2}: R\left(A^{1 / 2}\right) \rightarrow \mathcal{H}$ está densamente definido. Luego,

Proposición 2.4.6. Sea $B \in L(\mathcal{H})^{s}$ un operador inyectivo y supongamos que $(B, \mathcal{S})$ es compatible. Entonces,

$$
P_{B, \mathcal{S}}=A^{-1 / 2} P_{\mathcal{M} / / J(\mathcal{M})^{\perp}} A^{1 / 2} .
$$

Demostración. Como $B$ is inyectivo, aplicando la Proposición 2.1.3 tenemos que $\mathcal{P}(B, \mathcal{S})=$ $\left\{P_{B, \mathcal{S}}\right\}$. consideremos el operador $Q=A^{-1 / 2} P_{\mathcal{M} / / J(\mathcal{M})^{\perp}} A^{1 / 2}$. Recordemos que, por la Proposición 2.4.2, $P_{\mathcal{M} / / J(\mathcal{M})^{\perp}} A^{1 / 2}$ está bien definido y es acotado. Además, por el Corolario 2.4.4,

$$
R\left(P_{\mathcal{M} / / J(\mathcal{M})^{\perp}} A^{1 / 2}\right) \subseteq R\left(A^{1 / 2}\right),
$$

y en consecuencia $Q$ está bien definido. De la Ec. (2.4.2) tenemos que $P_{\mathcal{M} / / J(\mathcal{M})^{\perp}} A^{1 / 2}=$ $A^{1 / 2} P_{B, \mathcal{S}}$ y luego,

$$
Q=A^{-1 / 2} A^{1 / 2} P_{B, \mathcal{S}}=P_{B, \mathcal{S}}
$$


Podemos generalizar esta fórmula para el caso en que $B \in L(\mathcal{H})^{s}$ no es necesariamente inyectivo de la siguiente manera: dada la descomposición matricial inducida por el subespacio $\overline{R(B)}$ de $\mathcal{H}$,

$$
B=\left(\begin{array}{cc}
B_{R} & 0 \\
0 & 0
\end{array}\right)
$$

y el operador $B_{R}=\left.B\right|_{\overline{R(B)}} \in L(\overline{R(B)})^{s}$ es inyectivo. Definamos

$$
B^{\sharp}=\left(\begin{array}{cc}
B_{R}^{-1} & 0 \\
0 & 0
\end{array}\right),
$$

donde $B_{R}^{-1}: \overline{R(B)} \rightarrow \overline{R(B)}$ es la inversa densamente definida de $B_{R}$. Observemos que $B^{\sharp}$ es un operador lineal densamente definido. Más aún, si $R(B)$ es cerrado entonces $B^{\dagger}=B^{\sharp} P_{R(B)}$, siendo $B^{\dagger}$ la inversa generalizada de Moore-Penrose de $B$.

Proposición 2.4.7. Sean $B \in L(\mathcal{H})^{s}$ y $\mathcal{S}$ un subespacio cerrado de $\mathcal{H}$ tales que $(B, \mathcal{S})$ es compatible. Luego,

1. Si $\mathcal{S} \subseteq \overline{R(B)}$ entonces

$$
P_{B, \mathcal{S}}=\left(A^{1 / 2}\right)^{\sharp} P_{\mathcal{M} / / J(\mathcal{M}) \perp} A^{1 / 2} .
$$

2. Si $\mathcal{N}=\{0\}$ entonces

$$
P_{B, \mathcal{S}}=\left(P_{\overline{R(B)}} P_{\mathcal{S}}\right)^{\dagger} P_{B, P_{\overline{R(B)}}(\mathcal{S})}=\left(P_{\overline{R(B)}} P_{\mathcal{S}}\right)^{\dagger}\left(A^{1 / 2}\right)^{\sharp} P_{\mathcal{M} / / J(\mathcal{M})^{\perp}} A^{1 / 2} .
$$

Demostración. Notemos que, en ambos casos, $\mathcal{P}(B, \mathcal{S})=\left\{P_{B, \mathcal{S}}\right\}$ ya que $\mathcal{N}=\{0\}$.

1. Si $\mathcal{S} \subseteq \overline{R(B)}$, por el Corolario 2.4.4, podemos afirmar que el operador

$$
Q=\left(A^{1 / 2}\right)^{\sharp} P_{\mathcal{M} / / J(\mathcal{M})^{\perp}} A^{1 / 2}
$$

está bien definido. Por el Corolario 2.2.5, $P_{\mathcal{M} / / J(\mathcal{M})^{\perp}}\left(R\left(A^{1 / 2}\right)\right)=\mathcal{M} \cap R\left(A^{1 / 2}\right)=A^{1 / 2}(\mathcal{S})$ ya que $A^{1 / 2}(\mathcal{S})$ es cerrado en $R\left(A^{1 / 2}\right)$. Luego, $R(Q) \subseteq\left(A^{1 / 2}\right)^{\sharp} A^{1 / 2}(\mathcal{S})=\mathcal{S}$. Pero además, como $\mathcal{S} \subseteq \overline{R(B)}, Q x=x$ para todo $x \in \mathcal{S}$. En conclusión, $R(Q)=\mathcal{S}$. Por otra parte, $Q x=0$ si y sólo si $P_{\mathcal{M} / / J(\mathcal{M})^{\perp}} A^{1 / 2} x=0$, o equivalentemente, $x \in A^{-1 / 2}\left(J(\mathcal{M})^{\perp}\right)$, i.e. $N(Q)=B^{-1}\left(\mathcal{S}^{\perp}\right)$. Por lo tanto, $Q=P_{B, \mathcal{S}}$.

2. Si $(B, \mathcal{S})$ es compatible entonces, por la Proposición 2.2.2, el subespacio $\mathcal{T}=P_{\overline{R(B)}}(\mathcal{S})$ es cerrado y el par $(B, \mathcal{T})$ es compatible. Además, $\mathcal{T} \subseteq \overline{R(B)}$ y $\overline{A^{1 / 2}(\mathcal{T})}=\overline{A^{1 / 2}(\mathcal{S})}=\mathcal{M}$. Luego, aplicando el caso anterior, tenemos que $P_{B, \mathcal{T}}=\left(A^{1 / 2}\right)^{\sharp} P_{\mathcal{M} / / J(\mathcal{M})^{\perp}} A^{1 / 2}$.

Sólo resta probar entonces que $P_{B, \mathcal{S}}=\left(P_{\overline{R(B)}} P_{\mathcal{S}}\right)^{\dagger} P_{B, \mathcal{T}}$. Para ello, mostraremos que $P_{B, \mathcal{S}}$ es solución reducida de la ecuación

$$
\left(P_{\overline{R(B)}} P_{\mathcal{S}}\right) X=P_{B, \mathcal{T}},
$$

luego, por la unicidad de la misma, concluiremos que $P_{B, \mathcal{S}}=\left(P_{\overline{R(B)}} P_{\mathcal{S}}\right)^{\dagger} P_{B, \mathcal{T}}$.

Sea $Q=P_{\overline{R(B)}} P_{\mathcal{S}} P_{B, \mathcal{S}}=P_{\overline{R(B)}} P_{B, \mathcal{S}}$. Como $\mathcal{N}=\{0\}$, tenemos que $N\left(P_{B, \mathcal{S}}\right)=B(\mathcal{S})^{\perp} \supseteq$ $N(B)=N\left(P_{\overline{R(B)}}\right)$. Entonces $P_{B, \mathcal{S}} P_{\overline{R(B)}}=P_{B, \mathcal{S}} \mathrm{y}$

$$
Q^{2}=P_{\overline{R(B)}}\left(P_{B, \mathcal{S}} P_{\overline{R(B)}}\right) P_{B, \mathcal{S}}=P_{\overline{R(B)}} P_{B, \mathcal{S}}=Q .
$$


Además, $R(Q)=P_{\overline{R(B)}}(\mathcal{S})=\mathcal{T}$ y $B Q=B P_{B, \mathcal{S}}=P_{B, \mathcal{S}}^{*} B=\left(P_{B, \mathcal{S}}^{*} P_{\overline{R(B)}}\right) B=Q^{*} B$. Luego, $Q \in \mathcal{P}(B, \mathcal{T})=\left\{P_{B, \mathcal{T}}\right\}$, es decir, $Q=P_{B, \mathcal{T}}$. Por lo tanto, $P_{B, \mathcal{S}}$ es una solución de la Ec. (2.4.5).

Como $N\left(P_{B, \mathcal{S}}\right)=B(\mathcal{S})^{\perp}=B(\mathcal{T})^{\perp}=N\left(P_{B, \mathcal{T}}\right)$, tenemos también que $P_{B, \mathcal{S}}$ es una solución reducida de la Ec. (2.4.5). Por lo tanto, $P_{B, \mathcal{S}}=\left(P_{\overline{R(B)}} P_{\mathcal{S}}\right)^{\dagger} P_{B, \mathcal{T}}$.

En la sección 2.1.1 mostramos que, dados $B \in L(\mathcal{H})^{s}$ y $\mathcal{S}$ un subespacio cerrado de $\mathcal{H},(B, \mathcal{S})$ es compatible si y sólo si la ecuación

$$
G_{B, \mathcal{S}} X=P B
$$

admite una solución acotada (siendo $P$ la proyección ortogonal sobre $\mathcal{S}$ ).

Por el Corolario 1.1.3, la solución reducida es una proyección $Q \in L(\mathcal{H})$. Además, por ser la solución reducida de la Ec. (2.4.6), tenemos que $R(Q) \subseteq \overline{R\left(G_{B, \mathcal{S}}\right)}=\overline{R\left(G_{B, \mathcal{S} \ominus \mathcal{N})}\right.}=\mathcal{S} \ominus \mathcal{N}$ y $N(Q)=N(P B)=B^{-1}\left(\mathcal{S}^{\perp}\right)=B(\mathcal{S})^{\perp}$. Por lo tanto, $Q=P_{B, \mathcal{S} \ominus \mathcal{N}} \mathrm{y}$

$$
P_{B, \mathcal{S}}=P_{B, \mathcal{S} \ominus \mathcal{N}}+P_{\mathcal{N}}=Q+P_{\mathcal{N}}
$$

Proposición 2.4.8. Si el par $(B, \mathcal{S})$ es compatible, entonces la solución reducida de la ecuación

$$
(P B P) X=P B
$$

coincide con la solución reducida de

$$
\left(A^{1 / 2} P\right) X=P_{\mathcal{M} / / J(\mathcal{M})^{\perp}} A^{1 / 2} .
$$

Demostración. Por la Proposición 2.4.2, $P_{\mathcal{M} / / J(\mathcal{M})^{\perp}} A^{1 / 2}$ es un operador (bien definido) acotado. Luego, la Ec. (2.4.9) está bien definida y, por el Corolario 2.4.4, tiene una solución acotada.

Por la discusión que acompaña a la Ec. (2.4.6), la proyección $Q=P_{B, \mathcal{S} \ominus \mathcal{N}}$ es la solución reducida de la Ec. (2.4.8). Veamos que también es la solución reducida de la Ec. (2.4.9). Si $z \in \mathcal{S} \ominus \mathcal{N}$ entonces $\left(A^{1 / 2} P\right) Q z=A^{1 / 2} z=P_{\mathcal{M} / / J(\mathcal{M})^{\perp}} A^{1 / 2} z$. Por otra parte, si $z \in B(\mathcal{S} \ominus \mathcal{N})^{\perp}=$ $B(\mathcal{S})^{\perp}$ tenemos que $A^{1 / 2} z \in J(\mathcal{M})^{\perp}$ y entonces $P_{\mathcal{M} / / J(\mathcal{M})^{\perp}} A^{1 / 2} z=0=\left(A^{1 / 2} P\right) Q z$. Luego, $Q$ es una solución de (2.4.9). Más aún, $Q$ es la solución reducida porque

$$
R(Q)=\mathcal{S} \ominus \mathcal{N}=\left(\mathcal{S}^{\perp}+\mathcal{N}\right)^{\perp}=\left(\mathcal{S}^{\perp}+\mathcal{S} \cap N(B)\right)^{\perp}=N\left(A^{1 / 2} P\right)^{\perp}=\overline{R\left(\left(A^{1 / 2} P\right)^{*}\right)},
$$

lo que completa la demostración.

Supongamos ahora que $B \in L(\mathcal{H})^{s}$ es un operador de rango cerrado. $\mathrm{Si}(B, \mathcal{S})$ es compatible y $\mathcal{N}=\{0\}$, la demostración de la Proposición 2.2.12 sugiere la siguiente fórmula para $P_{B, \mathcal{S}}$ :

$$
P_{B, \mathcal{S}}=P G_{B, \mathcal{S}}^{\dagger} P B=P(P B P)^{\dagger} P B .
$$

Por su parte, si $\mathcal{N} \neq\{0\}$ tenemos que $P=P_{\mathcal{S} \ominus \mathcal{N}}+P_{\mathcal{N}}$. Luego, de la ecuación anterior concluímos que

$$
P_{B, \mathcal{S}}=P_{\mathcal{N}}+P(P B P)^{\dagger} P B .
$$

A continuación mostraremos que, en las fórmulas anteriores, podemos reemplazar la proyección ortogonal $P$ por cualquier otro operador $C \in L(\mathcal{H})$ con $R(C)=\mathcal{S}$, aún en el caso en que $B$ es un operador autoadjunto arbitrario. 
Proposición 2.4.9. Sea $B \in L(\mathcal{H})^{s}$ y consideremos un subespacio cerrado $\mathcal{S}$ de $\mathcal{H}$. Luego, $(B, \mathcal{S})$ es compatible si y sólo si $R\left(C^{*} B C\right)=R\left(C^{*} B\right)$ para todo $C \in L(\mathcal{H})$ con $R(C)=\mathcal{S}$.

Demostración. Que la igualdad entre los rangos es una condición suficiente para la compatibilidad del par $(B, \mathcal{S})$, es consecuencia de la Ec. (2.4.6), tomando $C=P_{\mathcal{S}}$, la proyección ortogonal sobre $\mathcal{S}$.

Recíprocamente, fijemos $C \in L(\mathcal{H})$ con $R(C)=\mathcal{S}$ y observemos que $C=P C$. Como $R(P B P)=R(P B)$, tenemos que

$$
R\left(C^{*} B C\right)=C^{*}(P B(\mathcal{S}))=C^{*}(R(P B P))=C^{*}(R(P B))=R\left(C^{*} B\right) .
$$

Proposición 2.4.10. Sean $B \in L(\mathcal{H})^{s}$ y $\mathcal{S}$ un subespacio cerrado de $\mathcal{H}$ tales que $(B, \mathcal{S})$ es compatible. Si consideramos un operador $C \in L(\mathcal{H})$ con $R(C)=\mathcal{S}$, entonces $P_{B, \mathcal{S} \ominus \mathcal{N}}=C D$, siendo $D$ la solución reducida de la ecuación

$$
\left(C^{*} B C\right) X=C^{*} B
$$

Demostración. en primer lugar, notemos que podemos suponer que $\mathcal{N}=\mathcal{S} \cap B(\mathcal{S})^{\perp}=\{0\}$ sin pérdida de generalidad. De hecho, como $(B, \mathcal{S})$ es compatible, $\mathcal{N}=\mathcal{S} \cap N(B)$ y, fijado un operador $C \in L(\mathcal{H})$ con $R(C)=\mathcal{S}, B C=B P_{\mathcal{S}} C=B\left(P_{\mathcal{S} \ominus \mathcal{N}}+P_{\mathcal{N}}\right) C=B P_{\mathcal{S} \ominus \mathcal{N}} C$. Entonces, si llamamos $C_{1}=P_{\mathcal{S} \ominus \mathcal{N}} C$, tenemos que $R\left(C_{1}\right)=\mathcal{S} \ominus \mathcal{N}, B C_{1}=B C$ y

$$
C_{1}^{*} B C_{1}=C_{1}^{*} B C=\left(B C_{1}\right)^{*} C=(B C)^{*} C=C^{*} B C .
$$

Por lo tanto, la ecuación $\left(C^{*} B C\right) X=C^{*} B$ puede reescribirse como $\left(C_{1}^{*} B C_{1}\right) X=C_{1}^{*} B$, con $R\left(C_{1}\right)=\mathcal{S} \ominus \mathcal{N}$ y $\mathcal{N}_{1}=(\mathcal{S} \ominus \mathcal{N}) \cap B(\mathcal{S} \ominus \mathcal{N})^{\perp}=(\mathcal{S} \ominus \mathcal{N}) \cap B(\mathcal{S})^{\perp}=\{0\}$.

Luego, fijemos $C \in L(\mathcal{H})$ con $R(C)=\mathcal{S}$ y supongamos que $\mathcal{N}=\{0\}$. Si $D \in L(\mathcal{H})$ es la solución reducida de la Ec. (2.4.12), entonces $R(D) \subseteq \overline{R\left(C^{*} B C\right)}$ y $N(D)=N\left(C^{*} B\right)=B(\mathcal{S})^{\perp}$. Además,

$$
\left(C^{*} B C\right) D C D=C^{*} B C D=C^{*} B
$$

y $R(D C D) \subseteq R(D) \subseteq \overline{R\left(C^{*} B C\right)}$. Como consecuencia de la unicidad de la solución reducida, tenemos que $D C D=D$. A su vez, esta igualdad asegura que $C D$ es una proyección con $N(C D)=N(D)=B(\mathcal{S})^{\perp}$.

Es claro que $R(C D) \subseteq R(C)=\mathcal{S}$ pero, dado que $\mathcal{H}=\mathcal{S} \dot{+} B(\mathcal{S})^{\perp}$ y $N(C D)=B(\mathcal{S})^{\perp}$, vemos que en realidad $R(C D)=\mathcal{S}$. Sólo resta probar entonces que $C D$ es $B$-autoadjunta, pero como

$$
(C D)^{*} B=D^{*}\left(C^{*} B\right)=D^{*}\left(C^{*} B C\right) D,
$$

tenemos que $(C D)^{*} B=B(C D)$, es decir, $C D$ es $B$-autoadjunta. Por lo tanto, $C D=P_{\mathcal{S} \ominus \mathcal{N}}$.

Dados $B \in L(\mathcal{H})^{s}$ y $\mathcal{S}$ un subespacio cerrado de $\mathcal{H}$ supongamos que $(B, \mathcal{S})$ es compatible. Si $D$ es la solución reducida de la Ec. (2.4.12) entonces, como $R(D) \subseteq \overline{R\left(C^{*} B C\right)}$, tenemos que $D=P_{\overline{R\left(C^{*} B C\right)}} D=\left(C^{*} B C\right)^{\sharp} C^{*} B$. Luego,

$$
P_{B, \mathcal{S} \ominus \mathcal{N}}=C D=C\left(C^{*} B C\right)^{\sharp} C^{*} B
$$


y, por la Ec. (2.4.7), vemos que

$$
P_{B, \mathcal{S}}=P_{\mathcal{N}}+C\left(C^{*} B C\right)^{\sharp} C^{*} B .
$$

Esta fórmula para la proyección minimal $P_{B, \mathcal{S}}$ generaliza la obtenida previamente por G. Corach et al. en [20, Proposición 3.6]. Recordemos que aquella sólo era válida en el caso en que $B$ fuera un operador positivo con rango cerrado. 


\section{Capítulo 3}

\section{Complementos de Schur en espacios de Hilbert}

En análisis matricial, el complemento de Schur de una matriz de bloques se define de la siguiente manera: dadas matrices $a, b, c, d$ de $p \times p, p \times q, q \times p$ y $q \times q$, respectivamente, supongamos que $a$ es inversible y consideremos la matriz $A$ de $(p+q) \times(p+q)$ definida por

$$
A=\left(\begin{array}{cc}
a & b \\
c & d
\end{array}\right)
$$

El complemento de Schur de la matriz $A$ (respecto al bloque $a$ ) es la matriz de $q \times q$ dada por

$$
A_{/ a}=d-c a^{-1} b \text {. }
$$

El complemento de Schur aparece naturalmente al resolver sistemas de ecuaciones lineales de la forma

$$
\left\{\begin{array}{l}
a u+b v=x \\
c u+d v=y
\end{array}\right.
$$

siendo $u$ y $x$ vectores columna $p$-dimensionales, $v$ e $y$ vectores columna $q$-dimensionales. Multiplicando la ecuación de arriba por $c a^{-1}$ y luego restando a la ecuación de abajo se obtiene

$$
\left(d-c a^{-1} b\right) v=y-c a^{-1} x .
$$

Entonces, si uno puede invertir la submatriz a y el complemento de Schur de $A$, uno puede resolver el sistema de ecuaciones. Es decir, el problema de resolver el sistema de $p+q$ ecuaciones (con $p+q$ incógnitas) se reduce a invertir dos matrices, una de $p \times p$ y otra de $q \times q$.

Si en cambio el bloque $a$ de la matriz $A$ no es inversible, D. Carlson et al. [13] definieron el complemento de Schur $A_{/ a}$ utilizando la inversa de Moore-Penrose de $a$ :

$$
A_{/ a}=d-c a^{\dagger} b .
$$

\subsection{Complemento de Schur de operadores positivos}

En 1971, W. N. Anderson Jr. [2] descubrió una nueva manifestación del complemento de Schur al estudiar el operador cortocircuito. La motivación para estudiar esta operación, así como 
también su nombre, provienen de la teoría de circuitos eléctricos: dado un circuito de $n=p+q$ puertos, con matriz de impedancia $A$, si los primeros $p$ puertos (es decir, los correspondientes al bloque $a$ ) son cortocircuitados, entonces el voltaje es forzado a permanecer en el subespacio $\{0\} \oplus \mathbb{C}^{q}$ - ya que no puede haber voltaje a lo largo de un circuito en corto - y es fácil ver que la nueva matriz de impedancia es

$$
A_{/ \mathcal{S}}=\left(\begin{array}{cc}
0 & 0 \\
0 & c-b^{*} a^{\dagger} b
\end{array}\right)
$$

siendo $a^{\dagger}$ la pseudoinversa de Moore-Penrose de $a$. En 1975, Anderson y G. E. Trapp [3] extendieron la noción de complemento de Schur (u operador cortocircuito) a espacios de Hilbert.

Definición (Krein [51], Anderson-Trapp [2] [3]). Sea $\mathcal{H}$ un espacio de Hilbert. Dados $A \in L(\mathcal{H})^{+}$ y un subespacio cerrado $\mathcal{S}$ de $\mathcal{H}$, el operador cortocircuito de $A$ a $\mathcal{S}$ está definido por

$$
A_{/ \mathcal{S}}=\operatorname{máx}_{\leq}\left\{X \in L(\mathcal{H})^{+}: X \leq A, R(X) \subseteq \mathcal{S}^{\perp}\right\},
$$

siendo $\leq$ el orden natural inducido por el cono $L(\mathcal{H})^{+}$.

Vale destacar que, dados un espacio de Hilbert $\mathcal{H}$, un operador $A \in L(\mathcal{H})^{+}$y un subespacio cerrado $\mathcal{S}$ de $\mathcal{H}$, la existencia del máximo (respecto al orden usual entre operadores autoadjuntos) del conjunto

$$
\mathcal{M}\left(A, \mathcal{S}^{\perp}\right)=\left\{X \in L(\mathcal{H})^{+}: X \leq A, R(X) \subseteq \mathcal{S}^{\perp}\right\}
$$

fue probada por M. G. Krein [52] en 1947, como parte de su teoría de extensiones autoadjuntas de operadores semiacotados simétricos.

Teorema 3.1.1 (M. G. Krein, [52]). Sea $\mathcal{H}$ un espacio de Hilbert. Dados $A \in L(\mathcal{H})^{+}$y un subespacio cerrado $\mathcal{S}$, el conjunto $\mathcal{M}\left(A, \mathcal{S}^{\perp}\right)$ posee un elemento máximo, respecto al orden $\leq$ inducido por el cono positivo $L(\mathcal{H})^{+}$.

Demostración. Sean $\mathcal{M}=\overline{A^{1 / 2}(\mathcal{S})}$ y $T=A^{1 / 2} P_{\mathcal{M}^{\perp}} A^{1 / 2}$. Notemos que, $T \in \mathcal{M}\left(A, \mathcal{S}^{\perp}\right)$. Si $X \in \mathcal{M}\left(A, \mathcal{S}^{\perp}\right)$ entonces $X \leq A$ y, por el Teorema de Douglas, existe una contracción $C \in L(\mathcal{H})$ tal que $X^{1 / 2}=A^{1 / 2} C$ y $R(C) \subseteq N(A)^{\perp}$. Luego,

$$
R(C) \subseteq A^{-1 / 2}\left(R\left(X^{1 / 2}\right)\right) \subseteq A^{-1 / 2}(\overline{R(X)}) \subseteq A^{-1 / 2}\left(\mathcal{S}^{\perp}\right)=\mathcal{M}^{\perp},
$$

y en consecuencia, $C C^{*} \leq P_{\mathcal{M}^{\perp}}$. Por lo tanto, $X=A^{1 / 2} C C^{*} A^{1 / 2} \leq A^{1 / 2} P_{\mathcal{M}^{\perp}} A^{1 / 2}=T$ i.e. $T=\operatorname{máx}_{\leq} \mathcal{M}\left(A, \mathcal{S}^{\perp}\right)$.

En esta sección recopilaremos algunos resultados sobre el complemento de Schur de operadores positivos. Para una exposición más amplia sobre este tema, derivamos al lector a los trabajos de Anderson y Trapp [3], E. L. Pekarev [59] y G. Corach et al. [19], [22].

En primer lugar, enumeraremos algunas consecuencias de la definición.

Proposición 3.1.2. Sean $A \in L(\mathcal{H})^{+}$y $\mathcal{S}$ un subespacio cerrado de $\mathcal{H}$. Luego,

1. $(\alpha A)_{/ \mathcal{S}}=\alpha A_{/ \mathcal{S}}$ para todo $\alpha \in \mathbb{C}$;

2. si $\mathcal{T}$ es un subespacio cerrado de $\mathcal{S}$ entonces $A_{/ \mathcal{T}} \leq A_{/ \mathcal{S}}$; 
3. si $B \in L(\mathcal{H})^{+}$y $B \leq A$ entonces $B_{/ \mathcal{S}} \leq A_{/ \mathcal{S}}$.

Notemos además que, como parte de la demostración del Teorema 3.1.1, hemos obtenido una fórmula para el complemento de Schur de $A$ a $\mathcal{S}$ :

$$
A_{/ \mathcal{S}}=A^{1 / 2} P_{\mathcal{M}^{\perp}} A^{1 / 2}
$$

siendo $\mathcal{M}=\overline{A^{1 / 2}(\mathcal{S})}$. La misma fue descubierta por Pekarev y Ju. L. Smul'jan en [60] y posteriormente utilizada exhaustivamente en [59].

A continuación, caracterizaremos el rango y el núcleo del complemento de Schur.

Proposición 3.1.3. Dados $A \in L(\mathcal{H})^{+}$y un subespacio cerrado $\mathcal{S}$ de $\mathcal{H}$, sea $\mathcal{M}=\overline{A^{1 / 2}(\mathcal{S})}$. Luego,

1. $R\left(\left(A_{/ \mathcal{S}}\right)^{1 / 2}\right)=R\left(A^{1 / 2}\right) \cap \mathcal{S}^{\perp}$

2. $R(A) \cap \mathcal{S}^{\perp} \subseteq R\left(A_{/ \mathcal{S}}\right) \subseteq R\left(A^{1 / 2}\right) \cap \mathcal{S}^{\perp}$;

3. $N\left(A_{/ \mathcal{S}}\right)=A^{-1 / 2}(\mathcal{M})$.

Demostración. En primer lugar, notemos que

$$
\begin{aligned}
R\left(A^{1 / 2}\right) \cap \mathcal{S}^{\perp} & =A^{1 / 2}\left(A^{-1 / 2}\left(\mathcal{S}^{\perp}\right)\right)=A^{1 / 2}\left(\mathcal{M}^{\perp}\right)=R\left(A^{1 / 2} P_{\mathcal{M}^{\perp}}\right)=R\left(\left(A^{1 / 2} P_{\mathcal{M}^{\perp}} A^{1 / 2}\right)^{1 / 2}\right)= \\
& =R\left(\left(A_{/ \mathcal{S}}\right)^{1 / 2}\right) .
\end{aligned}
$$

Los items 2. y 3. son un caso particular de la Proposición 3.3.5, la cual demostraremos más adelante.

El complemento de Schur de un operador positivo también puede caracterizarse como el ínfimo de un conjunto de operadores positivos. Mas precisamente,

Teorema 3.1.4. Dados $A \in L(\mathcal{H})^{+}$y un subespacio cerrado $\mathcal{S}$ de $\mathcal{H}$,

$$
A_{/ \mathcal{S}}=\inf \left\{Q^{*} A Q: Q \in \mathcal{Q}(\mathcal{H}), N(Q)=\mathcal{S}\right\} .
$$

Omitiremos la demostración de este resultado para no extendernos con los resultados preliminares. Más adelante probaremos que, si el par $(A, \mathcal{S})$ es compatible, entonces $A_{/ \mathcal{S}}$ es en realidad el mínimo de dicho conjunto.

A continuación presentaremos la fórmula de conmutatividad del complemento de Schur, para el caso en que pueda calcularse.

Proposición 3.1.5. Sean $A \in L(\mathcal{H})^{+}$y $\mathcal{S}$ un subespacio cerrado de $\mathcal{H}$. Luego, si $\mathcal{T}$ es un subespacio cerrado de $\mathcal{H}$ tal que $\mathcal{S}+\mathcal{T}$ es cerrado, entonces

$$
\left(A_{/ \mathcal{S}}\right) / \mathcal{T}=A_{/ \mathcal{S}+\mathcal{T}}=\left(A_{/ \mathcal{T}}\right) / \mathcal{S}
$$

Demostración. Supongamos que $\mathcal{T}$ es un subespacio cerrado de $\mathcal{H}$ tal que $\mathcal{S}+\mathcal{T}$ es cerrado. Para probar que $A_{/ \mathcal{S}+\mathcal{T}}=\left(A_{/ \mathcal{S}}\right)_{/ \mathcal{T}}$, mostraremos que los conjuntos $\mathcal{M}\left(A,(\mathcal{S}+\mathcal{T})^{\perp}\right)$ y $\mathcal{M}\left(A_{/ \mathcal{S}}, \mathcal{T}^{\perp}\right)$, definidos como en (3.1.2), son iguales. 
Si $X \in \mathcal{M}\left(A,(\mathcal{S}+\mathcal{T})^{\perp}\right)$ entonces $R(X) \subseteq(\mathcal{S}+\mathcal{T})^{\perp}=\mathcal{S}^{\perp} \cap \mathcal{T}^{\perp}$. Luego, tenemos que $X \leq A_{\mathcal{S}}$, ya que $X \leq A$ y $R(X) \subseteq \mathcal{S}^{\perp}$. Además, como $R(X) \subseteq \mathcal{T}^{\perp}$, vemos que $X \in \mathcal{M}\left(A_{/ \mathcal{S}}, \mathcal{T}^{\perp}\right)$. Recíprocamente, si $Y \in \mathcal{M}\left(A_{/ \mathcal{S}}, \mathcal{T}^{\perp}\right)$ entonces $Y \leq A_{/ \mathcal{S}}$, lo que implica que $R(Y) \subseteq R\left(Y^{1 / 2}\right) \subseteq$ $R\left(A_{/ \mathcal{S}}^{1 / 2}\right) \subseteq \overline{R(A / \mathcal{S})} \subseteq \mathcal{S}^{\perp}$. Como además $R(Y) \subseteq \mathcal{T}^{\perp}$, tenemos que $R(Y) \subseteq \mathcal{S}^{\perp} \cap \mathcal{T}^{\perp}=(\mathcal{S}+\mathcal{T})^{\perp}$. Finalmente, como $Y \leq A_{/ \mathcal{S}} \leq A$, tenemos que $Y \in \mathcal{M}\left(A,(\mathcal{S}+\mathcal{T})^{\perp}\right)$. Por lo tanto, $A_{/ \mathcal{S}+\mathcal{T}}=$ $\left(A_{/ \mathcal{S}}\right) / \mathcal{T}$.

De la misma forma puede probarse que $A_{/ \mathcal{S}+\mathcal{T}}=\left(A_{/ \mathcal{T}}\right)_{/ \mathcal{S}}$.

Si $\left(A_{n}\right)_{n \in \mathbb{N}}$ es una sucesión de operadores en $L(\mathcal{H})$, diremos que $\left(A_{n}\right)_{n \in \mathbb{N}}$ converge en la

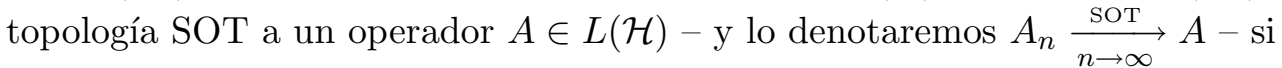

$$
\left\|A_{n} x-A x\right\| \underset{n \rightarrow \infty}{\longrightarrow} 0 \text { para todo } x \in \mathcal{H} \text {. }
$$

Además, si los operadores $\left(A_{n}\right)_{n \in \mathbb{N}}$ y $A$ son autoadjuntos, anotaremos $A_{n} \searrow^{\text {sOT }} A$ para indicar que $A_{n} \underset{n \rightarrow \infty}{\stackrel{\text { sot }}{\longrightarrow}} A$ y $A_{n} \geq A_{n+1}(\geq A)$ para todo $n \in \mathbb{N}$.

Los próximos resultados estudian la continuidad del complemento de Schur con respecto al límite SOT de operadores y al límite creciente de subespacios. Los mismos pueden encontrarse en $[3]$ y en $[6]$.

Proposición 3.1.6. Sean $A_{n}(n \in \mathbb{N})$ y A operadores en $L(\mathcal{H})^{+}$tales que $A_{n} \searrow^{\text {SOT }} A$ cuando $n \rightarrow \infty$. Entonces, $\left(A_{n}\right)_{/ \mathcal{S}} \searrow_{\text {SOT }} A_{/ \mathcal{S}}$ cuando $n \rightarrow \infty$, para todo subespacio cerrado $\mathcal{S}$ de $\mathcal{H}$.

Demostración. Como la sucesión $\left\{A_{n / \mathcal{S}}\right\}_{n \in \mathbb{N}}$ es decreciente, posee un límite en la topología fuerte de operadores, al cual llamaremos $L$. Dado que $A_{/ \mathcal{S}} \leq\left(A_{n}\right)_{/ \mathcal{S}} \leq A_{n}$ para todo $n \geq 1$, es claro que $A / \mathcal{S} \leq L \leq A$. Luego, basta verificar que $R(L) \subseteq \mathcal{S}^{\perp}$, pues en tal caso $L \in \mathcal{M}\left(A, \mathcal{S}^{\perp}\right)$ y, como $A_{/ \mathcal{S}}=\operatorname{máx}_{\leq} \mathcal{M}\left(A, \mathcal{S}^{\perp}\right)$, tendremos que $L=A_{/ \mathcal{S}}$.

Para probar que $R(L) \subseteq \mathcal{S}$, notemos que, por el Teorema de Douglas, la desigualdad $L \leq$ $\left(A_{n}\right)_{/ \mathcal{S}}$ implica que

$$
R(L) \subseteq R\left(L^{1 / 2}\right) \subseteq R\left(\left(A_{n}\right)_{/ \mathcal{S}}{ }^{1 / 2}\right) \subseteq \mathcal{S} .
$$

Por lo tanto, $\left(A_{n}\right) / \mathcal{S} \searrow_{\text {SOT }} A_{/ \mathcal{S}}$ cuando $n \rightarrow \infty$.

Proposición 3.1.7. Sean $\mathcal{S}_{n}(n \in \mathbb{N})$ y $\mathcal{S}$ subespacios cerrados tales que $P_{\mathcal{S}_{n}}{ }^{\text {SOT }} P_{\mathcal{S}}$ cuando $n \rightarrow \infty$. Entonces, $A_{/ \mathcal{S}_{n}} \searrow^{S O T} A_{/ \mathcal{S}}$ cuando $n \rightarrow \infty$, para todo $A \in L(\mathcal{H})^{+}$.

Demostración. Como $P_{\mathcal{S}_{n}}{ }^{\text {SOT }} P_{\mathcal{S}}$, tenemos que $\mathcal{S}_{n} \subseteq \mathcal{S}_{n+1} \subseteq \overline{\bigcup_{n \geq 1} \mathcal{S}_{n}}=\mathcal{S}$ para todo $n \in \mathbb{N}$. Luego, $A_{/ \mathcal{S}_{n}} \geq A_{/ \mathcal{S}_{n+1}} \geq A_{/ \mathcal{S}}$ para todo $n \geq 1$, es decir, $\left\{A_{/ \mathcal{S}_{n}}\right\}_{n \in \mathbb{N}}$ es una sucesión decreciente y, por lo tanto, posee un límite en la topología fuerte de operadores, al cual llamaremos $L$ y cumple $L \geq A / \mathcal{S}$.

Notemos además que, por el Teorema de Douglas, la desigualdad $L \leq A_{/ \mathcal{S}_{n}} \leq A$ implica que

$$
R(L) \subseteq R\left(L^{1 / 2}\right) \subseteq R\left(A_{/ \mathcal{S}_{n}}{ }^{1 / 2}\right) \subseteq \mathcal{S}_{n}^{\perp}, \quad \text { para todo } n \geq 1 .
$$

Entonces, $R(L) \subseteq \bigcap_{n \geq 1} \mathcal{S}_{n}^{\perp}=\mathcal{S}^{\perp}$. Por lo tanto, $L \in \mathcal{M}\left(A, \mathcal{S}^{\perp}\right)$ y, como $A_{/ \mathcal{S}}=\operatorname{máx}_{\leq} \mathcal{M}\left(A, \mathcal{S}^{\perp}\right)$, tenemos que $L=A_{/ \mathcal{S}}$. 
El siguiente ejemplo muestra que $P_{\mathcal{S}_{n}} \searrow_{\text {SOT }} P_{\mathcal{S}}$ no es una condición suficiente para asegurar la convergencia de la sucesión $\left(A_{/ \mathcal{S}_{n}}\right)_{n \in \mathbb{N}}$ a $A_{/ \mathcal{S}}$.

Ejemplo 3.1.8. Sea $A \in L(\mathcal{H})^{+}$tal que $N(A)=\{0\}$ y $R(A)$ no es cerrado. Consideremos un subespacio denso $\mathcal{T}$ de $\mathcal{H}$ tal que $\mathcal{T} \cap R\left(A^{1 / 2}\right)=\{0\}$ y sea $\left\{e_{n}\right\}_{n \in \mathbb{N}}$ una base ortonormal de $\mathcal{H}$ contenida en $\mathcal{T}$.

Llamando $\mathcal{S}_{n}=\overline{\operatorname{span}\left\{e_{k}: k \geq n\right\}}$ para $n \geq 1$, vemos que $P_{\mathcal{S}_{n}} \searrow^{\text {SOT }} 0$. Además, $A_{/ \mathcal{S}_{n}}=0$ para todo $n \in \mathbb{N}$, porque

$$
R\left(\left(A_{/ \mathcal{S}_{n}}\right)^{1 / 2}\right)=R\left(A^{1 / 2}\right) \cap \mathcal{S}_{n}^{\perp}=R\left(A^{1 / 2}\right) \cap \operatorname{span}\left\{e_{1}, \ldots, e_{n}\right\}=\{0\} .
$$

Sin embargo, $A_{/\{0\}}=A \neq 0$.

\subsection{Complemento de Schur de operadores autoadjuntos}

En [5], T. Ando propuso una definición para el complemento de Schur de un operador arbitrario $T \in L(\mathcal{H})$ bajo la hipótesis de que éste sea complementable respecto al subespacio en cuestión.

Definición (T. Ando). Dados un operador $T \in L(\mathcal{H})$ y un subespacio cerrado $\mathcal{S}$ de $\mathcal{H}$, diremos que $T$ es $\mathcal{S}$-complementable si existen dos operadores $M_{l}, M_{r} \in L(\mathcal{H})$ tales que:

1. $M_{l} P=M_{l}$ y $M_{l} T P=T P$;

2. $P M_{r}=M_{r}$ y $P T M_{r}=P T$;

donde $P=P_{\mathcal{S}}$ es la proyección ortogonal sobre $\mathcal{S}$.

Si $T \in L(\mathcal{H})$ es un operador $\mathcal{S}$-complementable, puede existir más de un par de operadores $\left(M_{l}, M_{r}\right)$ que cumplan con los items de la definición anterior. Sin embargo, los productos $T M_{r}$ y $M_{l} T$ sólo dependen de $T$ y $\mathcal{S}$. De hecho, tenemos que

$$
T M_{r}=M_{l} T M_{r}=M_{l} T,
$$

ya que, como consecuencia de la definición, $T M_{r}=T P M_{r}=M_{l} T P M_{r}=M_{l} T M_{r}=M_{l} P T M_{r}=$ $M_{l} P T=M_{l} T$. Luego, como el miembro izquierdo de la Ec. (3.2.1) no depende de $M_{l}$ (y el derecho no depende de $M_{r}$ ), hemos demostrado la afirmación.

Definición (T. Ando). Dado un subespacio cerrado $\mathcal{S}$ de $\mathcal{H}$ y un operador $\mathcal{S}$-complementable $T \in L(\mathcal{H})$, definimos la compresión de Schur $T_{\mathcal{S}}$ de $T$ a $\mathcal{S}$ y el complemento de Schur (generalizado) $T_{/ \mathcal{S}}$ de $T$ a $\mathcal{S}$, respectivamente, mediante

$$
T_{\mathcal{S}}:=T M_{r} \quad \text { y } \quad T_{/ \mathcal{S}}:=T-T M_{r} .
$$

Para justificar la terminología que utilizamos, consideremos la representación matricial de $T$ inducida por el subespacio $\mathcal{S}$

$$
T=\left(\begin{array}{ll}
a & b \\
c & d
\end{array}\right)
$$


y supongamos que el operador $a \in L(\mathcal{S})$ es inversible. Luego, si consideramos los operadores

$$
M_{r}=\left(\begin{array}{cc}
I & a^{-1} b \\
0 & 0
\end{array}\right) \quad \text { y } \quad M_{l}=\left(\begin{array}{cc}
I & 0 \\
c a^{-1} & 0
\end{array}\right)
$$

estos satisfacen la definición de $\mathcal{S}$-complementabilidad con $T$ y, en este caso,

$$
T_{/ \mathcal{S}}=T\left(I-M_{r}\right)=\left(\begin{array}{cc}
a & b \\
c & d
\end{array}\right)\left(\begin{array}{cc}
0 & -a^{-1} b \\
0 & I
\end{array}\right)=\left(\begin{array}{cc}
0 & 0 \\
0 & d-c a^{-1} b
\end{array}\right) .
$$

Esta expresión claramente nos recuerda a la definición del complemento de Schur de una matriz de bloques, mencionada en la Ec. (3.0.1).

Por otra parte, en [22, Proposición 3.1] G. Corach et al. mostraron que, dado un subespacio cerrado $\mathcal{S}$ de $\mathcal{H}$, la $\mathcal{S}$-compatibilidad y la $\mathcal{S}$-complementabilidad son propiedades equivalentes para operadores autoadjuntos.

Proposición 3.2.1. Dados $B \in L(\mathcal{H})^{s}$ y un subespacio cerrado $\mathcal{S}$ de $\mathcal{H}$, el par $(B, \mathcal{S})$ es compatible si y sólo si $B$ es $\mathcal{S}$-complementable.

Demostración. Supongamos que $(B, \mathcal{S})$ es compatible y que $Q \in \mathcal{P}(B, \mathcal{S})$. Luego, si $P$ es la proyección ortogonal sobre $\mathcal{S}$,

$$
P Q=Q \quad \text { y } P B=P B Q+P B(I-Q)=P B Q+P(I-Q)^{*} B=P B Q
$$

ya que $R\left((I-Q)^{*}\right)=N(I-Q)^{\perp}=\mathcal{S}^{\perp}=N(P)$. Por su parte, $Q^{*} P=Q^{*}$ y $Q^{*} B P=(B Q) P=$ $B P$. Entonces, vemos que $M_{r}=Q$ y $M_{l}=Q^{*}$ satisfacen la definición de $\mathcal{S}$-complementabilidad. Recíprocamente, si $B$ es $\mathcal{S}$-complementable y $M_{r} \in L(\mathcal{H})$ satisface el item 2. de la definición,

$$
R(P B)=R\left(P B M_{r}\right)=R\left(P B P M_{r}\right)=P B P\left(R\left(M_{r}\right)\right) \subseteq R(P B P) .
$$

Por lo tanto, aplicando la Proposición 2.1.7, tenemos que el par $(B, \mathcal{S})$ es compatible.

En la demostración anterior hemos visto que, si $(B, \mathcal{S})$ es compatible y $Q \in \mathcal{P}(B, \mathcal{S})$, entonces $M_{r}=Q$ y $M_{l}=Q^{*}$ satisfacen la definición de $\mathcal{S}$-complementabilidad. Por lo tanto, si $Q \in$ $\mathcal{P}(B, \mathcal{S})$

$$
B_{\mathcal{S}}=B Q \quad \text { and } \quad B / \mathcal{S}=B(I-Q),
$$

y estos operadores resultan autoadjuntos ya que $Q$ es una proyección $B$-autoadjunta.

Observación 3.2.2. $\mathrm{Si}(B, \mathcal{S})$ es compatible y $B=\left(\begin{array}{cc}a & b \\ b^{*} & c\end{array}\right)$, la representación matricial de $B / \mathcal{S}$ inducida por $\mathcal{S}$ nos recuerda a la Ec. (3.1.1), ya que, por la Observación 2.1.19:

$$
B_{/ \mathcal{S}}=B\left(I-P_{B, \mathcal{S}}\right)=\left(\begin{array}{cc}
a & b \\
b^{*} & c
\end{array}\right)\left(\begin{array}{cc}
0 & -a^{\dagger} b \\
0 & I
\end{array}\right)=\left(\begin{array}{cc}
0 & 0 \\
0 & c-b^{*} a^{\dagger} b
\end{array}\right)
$$




\subsubsection{Extremalidad del complemento de Schur a subespacios $B$-definidos}

Recordemos que si $A \in L(\mathcal{H})^{+}$el complemento de Schur de $A$ a $\mathcal{S}$ puede caracterizarse como

$$
A_{/ \mathcal{S}}=\inf \left\{Q^{*} A Q: Q \in \mathcal{Q}, N(Q)=\mathcal{S}\right\},
$$

(ver Teorema 3.1.4 y [3]). Más aún, G. Corach et al. [19] probaron que:

Proposición 3.2.3. Dados $A \in L(\mathcal{H})^{+}$y un subespacio cerrado $\mathcal{S}$ de $\mathcal{H}$, si $(A, \mathcal{S})$ es compatible entonces

$$
A_{/ \mathcal{S}}=\min \left\{Q^{*} A Q: Q \in \mathcal{Q}, N(Q)=\mathcal{S}\right\} .
$$

Demostración. Teniendo en cuenta el comentario anterior, sólo falta probar que $A_{/ \mathcal{S}}$ pertenece al conjunto en cuestión. Pero, si $Q=I-P_{A, \mathcal{S}}$, la Ec. (3.2.2) asegura que $A_{/ \mathcal{S}}=A Q=Q^{*} A Q$ y $N(Q)=\mathcal{S}$. Por lo tanto, $A_{/ \mathcal{S}}=\operatorname{mín}\left\{Q^{*} A Q: Q \in \mathcal{Q}, N(Q)=\mathcal{S}\right\}$.

A continuación nos proponemos generalizar la fórmula (3.2.3) para operadores $B \in L(\mathcal{H})^{s}$ y subespacios cerrados $\mathcal{S}$ de $\mathcal{H}$ que sean $B$-definidos y tales que el par $(B, \mathcal{S})$ resulte compatible. A pesar de que los resultados de estos párrafos estan enunciados para ambas clases de subespacios $B$-definidos, sólo presentaremos las demostraciones para el caso $B$-no negativo.

Cabe destacar que parte de las Proposiciones 3.2.4 y 3.2.8 fueron probadas anteriormente. Más precisamente, como consecuencia de los resultados probados por P. Massey y D. Stojanoff en [54] se deduce que, dados $B \in L(\mathcal{H})^{s}$ y un subespacio $B$-no negativo $\mathcal{S}$ de $\mathcal{H}$, si $(B, \mathcal{S})$ es compatible entonces $B / \mathcal{S}$ puede representarse como

$$
B_{/ \mathcal{S}}=\min _{\leq}\left\{Q^{*} B Q: Q \in \mathcal{Q}, N(Q)=\mathcal{S}\right\} .
$$

Proposición 3.2.4. Dados $B \in L(\mathcal{H})^{s}$ y $\mathcal{S}$ un subespacio cerrado de $\mathcal{H}$, supongamos que $(B, \mathcal{S})$ es compatible. Luego,

1. $B / \mathcal{S}=\operatorname{mí}_{\leq}\left\{Q^{*} B Q: Q \in \mathcal{Q}, N(Q)=\mathcal{S}\right\}$ si y sólo si $\mathcal{S}$ es $B$-no negativo.

2. $B / \mathcal{S}=\operatorname{máx}_{\leq}\left\{Q^{*} B Q: Q \in \mathcal{Q}, N(Q)=\mathcal{S}\right\}$ si y sólo si $\mathcal{S}$ es $B$-no positivo.

Demostración. Supongamos que $(B, \mathcal{S})$ es compatible y $\mathcal{S}$ es $B$-no negativo. Por la Ec. (3.2.2), $B_{/ \mathcal{S}}=B\left(I-P_{B, \mathcal{S}}\right)=\left(I-P_{B, \mathcal{S}}\right)^{*} B\left(I-P_{B, \mathcal{S}}\right)$ y entonces $B_{/ \mathcal{S}} \in\left\{Q^{*} B Q: Q \in \mathcal{Q}, N(Q)=\mathcal{S}\right\}$. Por otro lado, dada $Q \in \mathcal{Q}$ con $N(Q)=\mathcal{S}$, consideremos la proyección $E=I-Q$. Luego,

$$
\begin{aligned}
\left\langle Q^{*} B Q x, x\right\rangle & =\langle B Q x, Q x\rangle=\langle x-E x, x-E x\rangle_{B}= \\
& =\left\langle P_{B, \mathcal{S}} x+\left(I-P_{B, \mathcal{S}}\right) x-E x, P_{B, \mathcal{S}} x+\left(I-P_{B, \mathcal{S}}\right) x-E x\right\rangle_{B}= \\
& =\left\langle\left(I-P_{B, \mathcal{S}}\right) x,\left(I-P_{B, \mathcal{S}}\right) x\right\rangle_{B}+\left\langle P_{B, \mathcal{S}} x-E x, P_{B, \mathcal{S}} x-E x\right\rangle_{B} \geq \\
& \geq\left\langle B_{/ \mathcal{S}} x, x\right\rangle,
\end{aligned}
$$

para todo $x \in \mathcal{H}$. Por lo tanto, $Q^{*} B Q \geq B / \mathcal{S}$ para toda $Q \in \mathcal{Q}$ con $N(Q)=\mathcal{S}$, es decir, $B_{/ \mathcal{S}}=\operatorname{mí}_{\leq}\left\{Q^{*} B Q: Q \in \mathcal{Q}, N(Q)=\mathcal{S}\right\}$.

Recíprocamente, supongamos que $B_{/ \mathcal{S}}=\min _{\leq}\left\{Q^{*} B Q: Q \in \mathcal{Q}, N(Q)=\mathcal{S}\right\}$. Notemos que, dados $x \in \mathcal{H} \backslash \mathcal{S}$ y $s \in \mathcal{S}$, existe $E \in \mathcal{Q}$ con $R(\stackrel{\leq}{E})=\mathcal{S}$ tal que $E x=s$. Entonces,

$$
\begin{aligned}
\left\langle B_{/ \mathcal{S}} x, x\right\rangle & \leq\langle B(I-E) x,(I-E) x\rangle=\langle B(x-s), x-s\rangle= \\
& =\left\langle B\left(\left(x-P_{B, \mathcal{S}} x\right)+\left(P_{B, \mathcal{S}} x-s\right)\right),\left(x-P_{B, \mathcal{S}} x\right)+\left(P_{B, \mathcal{S}} x-s\right)\right\rangle= \\
& =\left\langle B\left(x-P_{B, \mathcal{S}} x\right), x-P_{B, \mathcal{S}} x\right\rangle+\left\langle B\left(P_{B, \mathcal{S}} x-s\right), P_{B, \mathcal{S}} x-s\right\rangle= \\
& =\left\langle B{ }_{\mathcal{S}} x, x\right\rangle+\left\langle B\left(P_{B, \mathcal{S}} x\right), P_{B, \mathcal{S}} x-s\right\rangle, \quad \text { para todo } s \in \mathcal{S},
\end{aligned}
$$


i.e. $\left\langle B\left(P_{B, \mathcal{S}} x-s\right), P_{B, \mathcal{S}} x-s\right\rangle \geq 0$ para todo $s \in \mathcal{S}$, o equivalentemente, $\mathcal{S}$ es $B$-no negativo.

Proposición 3.2.5. Sea $B \in L(\mathcal{H})^{s}$ y supongamos que el par $(B, \mathcal{S})$ es compatible. Entonces, las siguientes condiciones son equivalentes:

1. $B_{/ \mathcal{S}} \in L(\mathcal{H})^{+}$

2. $N(Q)$ es B-no negativo para alguna proyección $Q \in \mathcal{P}(B, \mathcal{S})$;

3. $B^{-1}\left(\mathcal{S}^{\perp}\right)$ es un subespacio B-no negativo de $\mathcal{H}$.

Demostración. $1 \Leftrightarrow 2$ : Dada $Q \in \mathcal{P}(B, \mathcal{S})$, tenemos que $B_{/ \mathcal{S}}=B(I-Q)$. Si $x \in \mathcal{H}$,

$$
\langle B / \mathcal{S} x, x\rangle=\langle B(I-Q) x, x\rangle=\langle(I-Q) x,(I-Q) x\rangle_{B} .
$$

Entonces, $B / \mathcal{S} \in L(\mathcal{H})^{+}$si y sólo si $R(I-Q)=N(Q)$ es $B$-no negativo.

$2 \Leftrightarrow 3$ : Notemos que $\mathcal{N}$ es un subespacio $B$-neutro de $\mathcal{H}$ y, dada cualquier proyección $Q \in$ $\mathcal{P}(B, \mathcal{S})$, resulta que $N(Q) \perp_{B} \mathcal{N}$ y $B^{-1}\left(\mathcal{S}^{\perp}\right)=N(Q) \dot{+} \mathcal{N}$. Por lo tanto, $N(Q)$ es $B$-no negativo si y sólo si $B^{-1}\left(\mathcal{S}^{\perp}\right)$ es $B$-no negativo.

Lo siguiente es una consecuencia directa de las Proposiciones 3.2.4 y 3.2.5.

Corolario 3.2.6. Sean $\mathcal{S}$ un subespacio cerrado de $\mathcal{H}$ y $B \in L(\mathcal{H})^{s}$ tales que el par $(B, \mathcal{S})$ es compatible. Luego, $B \in L(\mathcal{H})^{+}$si y sólo si $B_{/ \mathcal{S}} \in L(\mathcal{H})^{+}$y $\mathcal{S}$ es $B$-no negativo.

Observación 3.2.7. Recordemos que, dada una matriz de bloques autoadjunta

$$
A=\left(\begin{array}{cc}
a & b \\
b^{*} & c
\end{array}\right)
$$

el complemento de Schur de $M$ en $A$ está definido por

$$
A_{/ a}=c-b^{*} a^{\dagger} b
$$

donde $a^{\dagger}$ denota la inversa de Moore-Penrose de $a$. En [1], A. Albert mostró que $A \geq 0$ si y sólo si $a \geq 0, A_{/ a} \geq 0$ y $N(a) \subseteq N\left(b^{*}\right)$ (o equivalentemente, $R(b) \subseteq R(a)$ ). Luego, el corolario anterior puede interpretarse como una extensión de [1, Teorema 1]; recordemos que, por el Teorema 2.1.17, el par $(B, \mathcal{S})$ es compatible si y sólo si $R(b) \subseteq R(a)$, donde

$$
B=\left(\begin{array}{cc}
a & b \\
b^{*} & c
\end{array}\right)
$$

es la representación matricial de $B$ inducida por el subespacio $\mathcal{S}$.

El resultado de Albert es frecuentemente citado en la teoría de Control Robusto, ver por ejemplo, [12, Sección 2.1] y [31, Teorema 1.10]. Además, en [69, Corolario 2 (del Teorema 1.7)] puede encontrarse otra posible generalización de [1, Teorema 1].

Ahora, dados $B \in L(\mathcal{H})^{s}$ y un subespacio cerrado $\mathcal{S}$ de $\mathcal{H}$, consideremos los conjuntos

$$
\begin{aligned}
& \mathcal{M}^{-}\left(B, \mathcal{S}^{\perp}\right)=\left\{X \in L(\mathcal{H})^{s}: X \leq B, R(X) \subseteq \mathcal{S}^{\perp}\right\} \\
& \mathcal{M}^{+}\left(B, \mathcal{S}^{\perp}\right)=\left\{X \in L(\mathcal{H})^{s}: B \leq X, R(X) \subseteq \mathcal{S}^{\perp}\right\}
\end{aligned}
$$


Proposición 3.2.8. Supongamos que el par $(B, \mathcal{S})$ es compatible. Luego,

1. $\mathcal{S}$ es $B$-no negativo si y sólo si $B / \mathcal{S}=\operatorname{máx}_{\leq} \mathcal{M}^{-}\left(B, \mathcal{S}^{\perp}\right)$.

2. $\mathcal{S}$ es $B$-no positivo si y sólo si $B_{/ \mathcal{S}}=\min _{\leq} \mathcal{M}^{+}\left(B, \mathcal{S}^{\perp}\right)$.

Demostración. Supongamos que $\mathcal{S}$ es $B$-no negativo. Luego, si $Q=I-P_{B, \mathcal{S}}$, por la Proposición 2.3.7 tenemos que $Q$ es $B$-contractiva. Entonces, $B / \mathcal{S}=B Q=Q^{*} B Q \leq B$ y $R(B / \mathcal{S}) \subseteq \mathcal{S}^{\perp}$, i.e. $B_{/ \mathcal{S}} \in \mathcal{M}^{-}\left(B, \mathcal{S}^{\perp}\right)$. Mas aún, si $X \in \mathcal{M}^{-}\left(B, \mathcal{S}^{\perp}\right)$, vemos que $X=Q^{*} X=X Q=Q^{*} X Q \leq$ $Q^{*} B Q=B Q=B / \mathcal{S}$. Por lo tanto, $B / \mathcal{S}=\operatorname{máx}_{\leq} \mathcal{M}^{-}\left(B, \mathcal{S}^{\perp}\right)$.

Recíprocamente, supongamos que $B / \mathcal{S}=\operatorname{máx}_{\leq} \mathcal{M}^{-}\left(B, \mathcal{S}^{\perp}\right)$. Como

$$
R(B / \mathcal{S})=R\left(B\left(I-P_{B, \mathcal{S}}\right)\right)=B\left(N\left(P_{B, \mathcal{S}}\right)\right) \subseteq B\left(B^{-1}\left(\mathcal{S}^{\perp}\right)\right) \subseteq \mathcal{S}^{\perp}
$$

es fácil ver que $B_{/ \mathcal{S}}=Q^{*} B_{/ \mathcal{S}} Q \leq Q^{*} B Q$ para todo $Q \in \mathcal{Q}$ con $N(Q)=\mathcal{S}$. Por otra parte, $B_{/ \mathcal{S}}=\left(I-P_{B, \mathcal{S}}\right)^{*} B\left(I-P_{B, \mathcal{S}}\right)$ y $N\left(I-P_{B, \mathcal{S}}\right)=R\left(P_{B, \mathcal{S}}\right)=\mathcal{S}$. Entonces,

$$
B / \mathcal{S}=\min _{\leq}\left\{Q^{*} B Q: Q \in \mathcal{Q}, N(Q)=\mathcal{S}\right\}
$$

Luego, aplicando la Proposición 3.2.4, vemos que $\mathcal{S}$ es $B$-no negativo.

\subsubsection{El complemento de Schur a una suma de subespacios}

En esta sección, dados un operador autoadjunto $B \in L(\mathcal{H})$ y dos subespacios cerrados $\mathcal{S}_{1}$ y $\mathcal{S}_{2}$ de $\mathcal{H}$, nos interesa calcular (siempre que sea posible) el complemento de Schur a $\mathcal{S}_{2}$ de $B / \mathcal{S}_{1}$ y compararlo con el complemento de Schur a $\mathcal{S}_{1}$ de $B / \mathcal{S}_{2}$.

En primer lugar necesitamos probar que, si el par $(B, \mathcal{S})$ es compatible, el complemento de Schur $B / \mathcal{S}$ no depende de la parte $B$-isotrópica del subespacio $\mathcal{S}$. Más precisamente, probaremos que $B_{/ \mathcal{T}}=B_{/ \mathcal{S} \ominus \mathcal{N}}$ para todo $\mathcal{T}$ en una familia de subespacios de $\mathcal{H}$ (entre los que figura el subespacio $\mathcal{S})$.

Lema 3.2.9. Sean $B \in L(\mathcal{H})^{s}$ y $\mathcal{S}$ un subespacio cerrado de $\mathcal{H}$. Si $(B, \mathcal{S})$ es compatible y $\mathcal{T}$ es un subespacio cerrado de $\mathcal{H}$ tal que

$$
\mathcal{S} \ominus \mathcal{N} \subseteq \mathcal{T} \subseteq \mathcal{S}+N(B)
$$

entonces $(B, \mathcal{T})$ es compatible y $B_{/ \mathcal{T}}=B_{/ \mathcal{S} \ominus \mathcal{N}}$.

Demostración. Supongamos que $(B, \mathcal{S})$ es compatible. Luego, por la Proposición 2.1.4, el par $(B, \mathcal{S} \ominus \mathcal{N})$ es compatible. Notemos que, si $\mathcal{S} \ominus \mathcal{N} \subseteq \mathcal{T} \subseteq \mathcal{S}+N(B)$, entonces $B(\mathcal{T})=B(\mathcal{S} \ominus \mathcal{N})$ y consecuentemente $\mathcal{H}=\mathcal{S} \ominus \mathcal{N}+B(\mathcal{S})^{\perp} \subseteq \mathcal{T}+B(\mathcal{T})^{\perp}$. Luego, $(B, \mathcal{T})$ es compatible.

Veamos además que $B_{/ \mathcal{T}}=B / \mathcal{S} \ominus \mathcal{N}$. Como $R\left(I-P_{B, \mathcal{S} \ominus \mathcal{N}}\right)=N\left(P_{B, \mathcal{S} \ominus \mathcal{N}}\right)=B(\mathcal{S} \ominus \mathcal{N})^{\perp}=$ $B(\mathcal{T})^{\perp}=N\left(P_{B, \mathcal{T}}\right) \dot{+} \mathcal{T} \cap N(B)$, tenemos que $B P_{B, \mathcal{T}}\left(I-P_{B, \mathcal{S} \ominus \mathcal{N}}\right)=0$. Luego, $B_{\mathcal{T}}=B P_{B, \mathcal{T}}=$ $B P_{B, \mathcal{T}} P_{B, \mathcal{S} \ominus \mathcal{N}}=B P_{B, \mathcal{S} \ominus \mathcal{N}}=B_{\mathcal{S} \ominus \mathcal{N}}$ ya que $\mathcal{S} \ominus \mathcal{N} \subseteq \mathcal{T}$. Por lo tanto, $B_{/ \mathcal{T}}=B_{/ \mathcal{S} \ominus \mathcal{N}}$.

Proposición 3.2.10. Dado un operador $B \in L(\mathcal{H})^{s}$, consideremos dos subespacios cerrados de $\mathcal{H}, \mathcal{S}_{1}$ y $\mathcal{S}_{2}$, tales que $\mathcal{S}_{1} \perp_{B} \mathcal{S}_{2}$ y $\left(B, \mathcal{S}_{i}\right)$ es compatible para $i=1,2$. Entonces, el par $\left(B_{/ \mathcal{S}_{i}}, \mathcal{S}_{j}\right)$ es compatible $y$

$$
\mathcal{P}\left(B, \mathcal{S}_{j}\right)=\mathcal{P}\left(B / \mathcal{S}_{i}, \mathcal{S}_{j}\right) \quad(\text { para } i \neq j) .
$$


Demostración. Supongamos que $\left(B, \mathcal{S}_{i}\right)$ es compatible y consideremos una proyección $Q_{i} \in$ $\mathcal{P}\left(B, \mathcal{S}_{i}\right)$, para $i=1,2$. Como $\mathcal{S}_{1} \perp_{B} \mathcal{S}_{2}$, tenemos que $B Q_{i} Q_{j}=Q_{i}^{*} B Q_{j}=0$ si $i \neq j$. Entonces,

$$
B / \mathcal{S}_{i} Q_{j}=B\left(I-Q_{i}\right) Q_{j}=B Q_{j}\left(I-Q_{i}\right)=Q_{j}^{*} B_{\mathcal{S}_{i}},
$$

i.e. $Q_{j} \in \mathcal{P}\left(B / \mathcal{S}_{i}, \mathcal{S}_{j}\right)$. La otra inclusión se prueba de manera análoga.

Proposición 3.2.11. Dado un operador $B \in L(\mathcal{H})^{s}$, consideremos dos subespacios cerrados de $\mathcal{H}, \mathcal{S}_{1}$ y $\mathcal{S}_{2}$, tales que $\mathcal{S}_{1} \perp_{B} \mathcal{S}_{2}$ y $\mathcal{S}_{1} \cap \mathcal{S}_{2}=\{0\}$. Si $\mathcal{S}=\mathcal{S}_{1}+\mathcal{S}_{2}$ es cerrado y $\left(B, \mathcal{S}_{i}\right)$ es compatible para $i=1,2$, entonces $(B, \mathcal{S})$ es compatible $y$

$$
B / \mathcal{S}=\left(B / \mathcal{S}_{1}\right) / \mathcal{S}_{2}=\left(B / \mathcal{S}_{2}\right) / \mathcal{S}_{1} .
$$

Demostración. Considerando el subespacio $\mathcal{N}_{1}=\mathcal{S}_{1} \cap N(B)$, observemos que $\mathcal{N}_{1} \cap \mathcal{S}_{2}=\{0\}$ y que $\mathcal{N}_{1}+\mathcal{S}_{2}$ es un subespacio cerrado de $B\left(\mathcal{S}_{1}\right)^{\perp}$, esto último se debe a que $c_{0}\left(\mathcal{N}_{1}, \mathcal{S}_{2}\right) \leq$ $c_{0}\left(\mathcal{S}_{1}, \mathcal{S}_{2}\right)<1$. Si definimos $\mathcal{T}_{1}=B\left(\mathcal{S}_{1}\right)^{\perp} \ominus\left(\mathcal{N}_{1}+\mathcal{S}_{2}\right)$, entonces el subespacio $\mathcal{T}_{1}+\mathcal{S}_{2}$ es cerrado y se satisface la siguiente igualdad $\mathcal{T}_{1} \dot{+} \mathcal{N}_{1}+\mathcal{S}_{2}=B\left(\mathcal{S}_{1}\right)^{\perp}$. Si $Q_{1}=P_{\mathcal{S}_{1} / / \mathcal{T}_{1}+\mathcal{S}_{2}}$, notemos que $Q_{1} \in \mathcal{P}\left(B, \mathcal{S}_{1}\right)$ y $Q_{1} P=0$ para toda $P \in \mathcal{Q}$ con $R(P)=\mathcal{S}_{2}$.

Análogamente, consideremos $\mathcal{N}_{2}=\mathcal{S}_{2} \cap N(B), \mathcal{T}_{2}=B\left(\mathcal{S}_{2}\right)^{\perp} \ominus\left(\mathcal{N}_{2}+\mathcal{S}_{1}\right)$ y la proyección $Q_{2}=P_{\mathcal{S}_{2} / / \mathcal{T}_{2}+\mathcal{S}_{1}} \in \mathcal{P}\left(B, \mathcal{S}_{2}\right)$. Luego, $Q=Q_{1}+Q_{2} \in \mathcal{Q}$ es $B$-autoadjunta (pues $Q_{1}$ y $Q_{2}$ lo son) y $R(Q)=R\left(Q_{1}\right)+R\left(Q_{2}\right)=\mathcal{S}_{1}+\mathcal{S}_{2}=\mathcal{S}$, i.e. $(B, \mathcal{S})$ es compatible. Además, como $I-Q=\left(I-Q_{1}\right)\left(I-Q_{2}\right)$, tenemos que

$$
B_{/ \mathcal{S}}=B(I-Q)=B\left(I-Q_{1}\right)\left(I-Q_{2}\right)=B_{/ \mathcal{S}_{1}}\left(I-Q_{2}\right)=B_{/ \mathcal{S}_{1}}\left(I-Q_{2}\right)=\left(B_{/ \mathcal{S}_{1}}\right) / \mathcal{S}_{2} .
$$

De la misma manera, podemos probar que $B / \mathcal{S}=\left(B_{/ \mathcal{S}_{2}}\right) / \mathcal{S}_{1}$.

Para obtener una representación del complemento de Schur $B_{/ \mathcal{S}}$ para un subespacio cerrado $B$-indefinido $\mathcal{S}$, es necesario descomponer a $\mathcal{S}$ como $\mathcal{S}=\mathcal{S}_{+} \dot{+} \mathcal{S}_{-}$, siendo $\mathcal{S}_{+}$un subespacio $B$-no negativo y $\mathcal{S}_{-}$un subespacio $B$-no positivo - recordemos que descomposiciones de este tipo son posibles gracias al Teorema 2.3.2 del Capítulo 3 -. Utilizando la descomposición mencionada y la Proposición 3.2.11, puede probarse la siguiente caracterización de $B_{/ \mathcal{S}}$.

Proposición 3.2.12. Sean $\mathcal{S}$ un subespacio cerrado de $\mathcal{H}$ y $B \in L(\mathcal{H})^{s}$. Si $(B, \mathcal{S})$ es compatible entonces

$$
B_{/ \mathcal{S}}=\left(B / \mathcal{S}_{+}\right) / \mathcal{S}_{-}=\left(B / \mathcal{S}_{-}\right) / \mathcal{S}_{+},
$$

donde $\mathcal{S} \ominus \mathcal{N}=\mathcal{S}_{+} \oplus \mathcal{S}_{-}$es la descomposición dada en el Teorema 2.3.2.

Demostración. Si $(B, \mathcal{S})$ es compatible entonces, por el Lema 3.2 .9 , sabemos que $B_{/ \mathcal{S}}=B_{/ \mathcal{S} \ominus \mathcal{N}}$. Recordemos que, si $\mathcal{S} \ominus \mathcal{N}=\mathcal{S}_{+} \oplus \mathcal{S}_{-}$es la descomposición dada en el Teorema 2.3.2, los pares $\left(B, \mathcal{S}_{ \pm}\right)$son compatibles y $\mathcal{S}_{+} \perp_{B} \mathcal{S}_{-}$. Por lo tanto, aplicando la Proposición 3.2.11 tenemos que

$$
B_{/ \mathcal{S}}=\left(B / \mathcal{S}_{+}\right) / \mathcal{S}_{-}=\left(B_{/ \mathcal{S}_{-}}\right) / \mathcal{S}_{+} .
$$

Supongamos que el par $(B, \mathcal{S})$ es compatible y consideremos la descomposición del Teorema 2.3.2. Luego, como $\mathcal{S}_{+}$y $\mathcal{S}_{-}$son subespacios $B$-definidos, la Proposición 3.2.8 asegura que

$$
B / \mathcal{S}_{+} \leq B \leq B_{/ \mathcal{S}_{-}} .
$$

El siguiente corolario muestra que el complemento de Schur $B_{/ \mathcal{S}}$ también pertenece a este intervalo de operadores autoadjuntos. 
Corolario 3.2.13. Sean $B \in L(\mathcal{H})^{s}$ y $\mathcal{S}$ un subespacio cerrado de $\mathcal{H}$. Si $(B, \mathcal{S})$ es compatible entonces

$$
B / \mathcal{S}_{+} \leq B / \mathcal{S} \leq B / \mathcal{S}_{-},
$$

donde $\mathcal{S} \ominus \mathcal{N}=\mathcal{S}_{+} \oplus \mathcal{S}_{-}$es la descomposición dada en el Teorema 2.3.2.

Demostración. Si $(B, \mathcal{S})$ es compatible, consideremos la descomposición dada en el Teorema 2.3.2. Aplicando las Proposiciones 3.2.12 y 3.2.8, vemos que

$$
B_{/ \mathcal{S}}=\left(B_{/ \mathcal{S}_{-}}\right){/ \mathcal{S}_{+}}=\operatorname{máx}_{\leq} \mathcal{M}^{-}\left(B_{/ \mathcal{S}_{-}}, \mathcal{S}_{+}^{\perp}\right) \leq B_{/ \mathcal{S}_{-}}
$$

y, análogamente, $B / \mathcal{S}=\left(B / \mathcal{S}_{+}\right) / \mathcal{S}_{-}=\min _{\leq} \mathcal{M}^{+}\left(B_{/ \mathcal{S}_{+}}, \mathcal{S}_{-}^{\perp}\right) \geq B / \mathcal{S}_{+}$.

\subsubsection{Una representación minimax respecto a subespacios $B$-indefinidos}

A partir de la expresión obtenida en la Proposición 3.2.12 para el complemento de Schur de un operador autoadjunto $B \in L(\mathcal{H})$ a un subespacio $B$-indefinido $\mathcal{S}$ de $\mathcal{H}$ :

$$
B_{/ \mathcal{S}}=\left(B_{/ \mathcal{S}_{+}}\right) / \mathcal{S}_{-},
$$

mostraremos que $B_{/ \mathcal{S}}$ admite una representación del tipo minimax, es decir, es el mínimo entre el conjunto de máximos obtenidos al variar adecuadamente cierto conjunto de operadores.

Teorema 3.2.14. Sean $B \in L(\mathcal{H})^{s}$ y $\mathcal{S}$ un subespacio cerrado de $\mathcal{H}$. Si $(B, \mathcal{S})$ es compatible entonces

$$
B_{\mathcal{S}}=\operatorname{mín}_{N\left(Q_{+}\right)=\mathcal{S}_{+}} \operatorname{máx}_{N\left(Q_{-}\right)=\mathcal{S}_{-}} Q_{+}^{*}\left(Q_{-}^{*} B Q_{-}\right) Q_{+}=\operatorname{máx}_{N\left(Q_{-}\right)=\mathcal{S}_{-}} \operatorname{mín}_{\left(Q_{+}\right)=\mathcal{S}_{+}} Q_{-}^{*}\left(Q_{+}^{*} B Q_{+}\right) Q_{-},
$$

donde $\mathcal{S} \ominus \mathcal{N}=\mathcal{S}_{+} \oplus \mathcal{S}_{-}$es la descomposición dada en el Teorema 2.3.2.

Demostración. Si $(B, \mathcal{S})$ es compatible entonces, por el Teorema 2.3.2, $\mathcal{S} \ominus \mathcal{N}=\mathcal{S}_{+} \oplus \mathcal{S}_{-}$, siendo $\mathcal{S}_{+}$un subespacio (cerrado) $B$-positivo y $\mathcal{S}_{-}$un subespacio (cerrado) $B$-negativo tales que $\left(B, \mathcal{S}_{ \pm}\right)$es compatible y $\mathcal{S}_{+} \perp_{B} \mathcal{S}_{-}$. Luego, aplicando las Proposiciones 3.2.12 y 3.2.4, tenemos que

$$
\begin{aligned}
B / \mathcal{S} & =\left(B / \mathcal{S}_{-}\right) / \mathcal{S}_{+}=\min _{N\left(Q_{+}\right)=\mathcal{S}_{+}} Q_{+}^{*} B / \mathcal{S}_{-} Q_{+}=\operatorname{mín}_{N\left(Q_{+}\right)=\mathcal{S}_{+}} Q_{+}^{*}\left(\operatorname{máx}_{N\left(Q_{-}\right)=\mathcal{S}_{-}} Q_{-}^{*} B Q_{-}\right) Q_{+}= \\
& =\operatorname{mín}_{N\left(Q_{+}\right)=\mathcal{S}_{+}}\left(\operatorname{máx}_{N\left(Q_{-}\right)=\mathcal{S}_{-}} Q_{+}^{*} Q_{-}^{*} B Q_{-} Q_{+}\right) .
\end{aligned}
$$

Análogamente, $B / \mathcal{S}=\left(B_{/ \mathcal{S}_{+}}\right) / \mathcal{S}_{-}=\operatorname{máx}_{N\left(Q_{-}\right)=\mathcal{S}_{-}}\left(\operatorname{mín}_{N\left(Q_{+}\right)=\mathcal{S}_{+}} Q_{-}^{*}\left(Q_{+}^{*} B Q_{+}\right) Q_{-}\right)$.

El siguiente corolario es una interesante consecuencia de este teorema ya que, fijado un vector $x \in \mathcal{H}$, caracteriza el valor $\left\langle B_{/ \mathcal{S}} x, x\right\rangle$ como un minimax.

Corolario 3.2.15. Sean $B \in L(\mathcal{H})^{s}$ y $\mathcal{S}$ un subespacio cerrado de $\mathcal{H}$. Si el par $(B, \mathcal{S})$ es compatible entonces, para todo $x \in \mathcal{H}$,

$$
\langle B / \mathcal{S} x, x\rangle=\min _{s \in \mathcal{S}_{+}} \operatorname{máx}_{t \in \mathcal{S}_{-}}\langle B(x-(s+t)), x-(s+t)\rangle,
$$

donde $\mathcal{S} \ominus \mathcal{N}=\mathcal{S}_{+} \oplus \mathcal{S}_{-}$es la descomposición dada en el Teorema 2.3.2. 
Demostración. Por el Teorema 3.2.14, sabemos que

$$
B_{/ \mathcal{S}}=\min _{N\left(Q_{+}\right)=\mathcal{S}_{+}} \operatorname{máx}_{N\left(Q_{-}\right)=\mathcal{S}_{-}} Q_{+}^{*}\left(Q_{-}^{*} B Q_{-}\right) Q_{+}=\operatorname{mín}_{N\left(Q_{+}\right)=\mathcal{S}_{+}} Q_{+}^{*} B / \mathcal{S}_{-} Q_{+} .
$$

Por lo tanto, fijado $x \in \mathcal{H},\langle B / \mathcal{S} x, x\rangle \leq\left\langle Q_{+}^{*} B_{/ \mathcal{S}_{-}} Q_{+} x, x\right\rangle$ para toda $Q_{+} \in \mathcal{Q}$ con $N\left(Q_{+}\right)=$ $\mathcal{S}_{+}$. Además, como $B_{/ \mathcal{S}}=\left(I-P_{B, \mathcal{S}_{+}}\right)^{*} B_{/ \mathcal{S}_{-}}\left(I-P_{B, \mathcal{S}_{+}}\right)$y $N\left(I-P_{B, \mathcal{S}_{+}}\right)=\mathcal{S}_{+}$, vemos que $\left\langle B_{/ \mathcal{S}} x, x\right\rangle=\min _{N\left(Q_{+}\right)=\mathcal{S}_{+}}\left\langle Q_{+}^{*} B_{\mathcal{S}_{-}} Q_{+} x, x\right\rangle$. Luego,

$$
\begin{aligned}
\left\langle B_{/ \mathcal{S}} x, x\right\rangle & =\min _{N\left(Q_{+}\right)=\mathcal{S}_{+}}\left\langle Q_{+}^{*} B_{\mathcal{S}_{-}} Q_{+} x, x\right\rangle= \\
& =\min _{N\left(Q_{+}\right)=\mathcal{S}_{+}}\left\langle B_{/ \mathcal{S}_{-}}\left(x-\left(I-Q_{+}\right) x\right), x-\left(I-Q_{+}\right) x\right\rangle=\min _{s \in \mathcal{S}_{+}}\left\langle B_{/ \mathcal{S}_{-}}(x-s), x-s\right\rangle .
\end{aligned}
$$

Como $\mathcal{S}_{-}$es un subespacio $B$-negativo, por la Proposición 3.2.4 tenemos que

$$
B / \mathcal{S}_{-}=\operatorname{máx}_{N\left(Q_{-}\right)=\mathcal{S}_{-}} Q_{-}^{*} B Q_{-}
$$

y un argumento similar al anterior muestra que

$$
\langle B / \mathcal{S} x, x\rangle=\min _{s \in \mathcal{S}_{+}}\left\langle B_{\mathcal{S}_{-}}(x-s), x-s\right\rangle=\min _{s \in \mathcal{S}_{+}} \operatorname{máx}_{t \in \mathcal{S}_{-}}\langle B(x-(s+t)), x-(s+t)\rangle,
$$

para todo $x \in \mathcal{H}$.

\subsection{Una fórmula para el complemento de Schur}

En [59, Teorema 1.4], E. L. Pekarev probó que el complemento de Schur de un operador $A \in L(\mathcal{H})^{+}$a un subespacio cerrado $\mathcal{S}$ de $\mathcal{H}$ puede representarse mediante la siguiente fórmula:

$$
A_{/ \mathcal{S}}=A^{1 / 2} P_{\mathcal{M}^{\perp}} A^{1 / 2},
$$

donde $P_{\mathcal{M}^{\perp}}$ es la proyección ortogonal sobre $\mathcal{M}^{\perp}$ y $\mathcal{M}=\overline{A^{1 / 2}(\mathcal{S})}$.

En esta sección probaremos que, dados un operador $B \in L(\mathcal{H})^{s}$ y un subespacio cerrado $\mathcal{S}$ de $\mathcal{H}$ tales que $(B, \mathcal{S})$ es compatible, hay una generalización natural de la fórmula de Pekarev (3.3.1) para el complemento de Schur de $B$ a $\mathcal{S}$. Más aún, mostraremos que, si la fórmula dada define un operador acotado, la misma puede utilizarse para extender la definición de $B_{/ \mathcal{S}}$ en casos en los que el par $(B, \mathcal{S})$ no sea compatible, manteniendo las propiedades básicas del complemento de Schur.

En lo que sigue, dado un operador $B \in L(\mathcal{H})^{s}$ utilizaremos la descomposición polar de $B$ dada por $B=J A$, donde $A=|B| \in L(\mathcal{H})^{+}$y $J=J^{*}=J^{-1}$ cumple que $J x=x$ para todo $x \in N(B)$. Recordemos que, si el par $(B, \mathcal{S})$ es compatible y $\mathcal{M}=\overline{A^{1 / 2}(\mathcal{S})}$, la proyección $P_{\mathcal{M} / / J(\mathcal{M})^{\perp}}$ está densamente definida pero el producto $P_{\mathcal{M} / / J(\mathcal{M})^{\perp}} A^{1 / 2}$ permanece acotado (ver la Proposición 2.4.2).

Teorema 3.3.1. Sean $B \in L(\mathcal{H})^{s}$ y $\mathcal{S}$ un subespacio cerrado de $\mathcal{H}$ tales que el par $(B, \mathcal{S})$ es compatible. Si $B=J A$ es la descomposición polar de $B$, con $A \in L(\mathcal{H})^{+}$y $J=J^{*}=J^{-1}$, la compresión a $\mathcal{S}$ y el complemento de Schur de $B$ a $\mathcal{S}$ pueden calcularse como

$$
B_{\mathcal{S}}=J A^{1 / 2} P_{\mathcal{M} / / J(\mathcal{M}) \perp} A^{1 / 2} \quad \text { y } \quad B_{/ \mathcal{S}}=J A^{1 / 2} P_{J(\mathcal{M}) \perp / / \mathcal{M}} A^{1 / 2}
$$

siendo $\mathcal{M}=\overline{A^{1 / 2}(\mathcal{S})}$. 
Demostración. $\mathrm{Si}(B, \mathcal{S})$ es compatible entonces, por el Corolario 2.2.6, la proyección $P_{\mathcal{M} / / J(\mathcal{M})^{\perp}}$ está densamente definida. Más aún, la composición $P_{\mathcal{M} / / J(\mathcal{M})^{\perp}} A^{1 / 2}$ está bien definida y resulta un operador acotado que satisface

$$
P_{\mathcal{M} / / J(\mathcal{M})^{\perp}} A^{1 / 2}=A^{1 / 2} Q,
$$

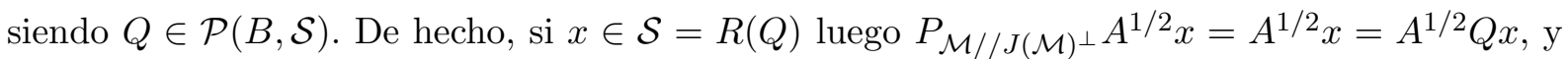

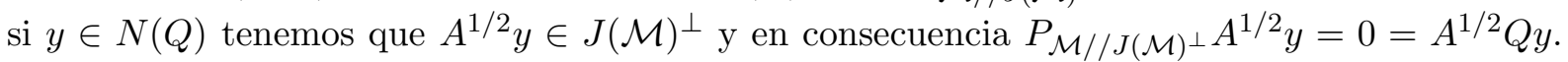
Por lo tanto, si $Q \in \mathcal{P}(B, \mathcal{S})$ vemos que $B_{\mathcal{S}}=B Q=J A^{1 / 2}\left(A^{1 / 2} Q\right)=J A^{1 / 2} P_{\mathcal{M} / / J(\mathcal{M}) \perp} A^{1 / 2}$ y

$$
B_{/ \mathcal{S}}=B-B_{\mathcal{S}}=J A^{1 / 2} P_{J(\mathcal{M}) \perp / / \mathcal{M}} A^{1 / 2} .
$$

En particular, si $B \in L(\mathcal{H})^{+}$, la fórmula (3.3.2) para el operador cortocircuito de $B$ a $\mathcal{S}$ coincide con la fórmula de Pekarev [59, Teorema 1.4]. El próximo ejemplo muestra que la Ec. (3.3.2) proporciona operadores bien definidos y acotados aún en algunos casos en los que la definición del complemento de Schur dada por T. Ando en [5] no puede aplicarse.

Ejemplo 3.3.2. Sean $\mathcal{S}$ y $\mathcal{T}$ dos subespacios cerrados de un espacio de Hilbert $\mathcal{H}$ (de dimensión infinita) tales que $\mathcal{S}+\mathcal{T}$ no es cerrado. Denotemos por $\mathcal{W}$ al complemento ortogonal de $\mathcal{T}$ en $\mathcal{H}$ y consideremos el espacio de Hilbert $\mathcal{H}_{2}=\mathcal{H} \oplus \mathcal{H}$ y los operadores $A, J \in L\left(\mathcal{H}_{2}\right)$ definidos por

$$
A=\left(\begin{array}{cc}
P_{\mathcal{W}} & 0 \\
0 & I
\end{array}\right), \quad J=\left(\begin{array}{cc}
I & 0 \\
0 & -I
\end{array}\right)
$$

en la representación matricial inducida por el subespacio $\mathcal{H} \oplus\{0\}$. Notemos que $A \in L\left(\mathcal{H}_{2}\right)^{+}$, su rango $R(A)=\mathcal{W} \oplus \mathcal{H}$ es cerrado y $J$ es una simetría i.e. $J=J^{*}=J^{-1}$. Luego, como $J$ y $A$ conmutan, el operador $B=J A$ es autoadjunto, $A=|B|$ y $J$ es el operador unitario de la descomposición polar de $B$.

Dado el subespacio cerrado $\mathcal{S}_{2}=\mathcal{S} \oplus\{0\}$ de $\mathcal{H}_{2}$, mostraremos que los operadores dados por la Ec. (3.3.2) están bien definidos y sin embargo el par $\left(B, \mathcal{S}_{2}\right)$ no es compatible.

Observemos que $\mathcal{M}=\overline{A^{1 / 2}\left(\mathcal{S}_{2}\right)}=\overline{P_{\mathcal{W}}(\mathcal{S})} \oplus\{0\} \subseteq \mathcal{H} \oplus\{0\}$ es un subespacio invariante por $J$ y en consecuencia $P_{J(\mathcal{M}) \perp / / \mathcal{M}}=P_{\mathcal{M}^{\perp}} \in L\left(\mathcal{H}_{2}\right)$. Por lo tanto, el operador $B / \mathcal{S}_{2}$ (dado por la Ec. (3.3.2)) está definido, de hecho tenemos su representación matricial -inducida por $\mathcal{H} \oplus\{0\}-$,

$$
B_{/ \mathcal{S}_{2}}=\left(\begin{array}{cc}
P_{\mathcal{W}} P_{\mathcal{Z}} P_{\mathcal{W}} & 0 \\
0 & -I
\end{array}\right)
$$

siendo $\mathcal{Z}$ el complemento ortogonal del subespacio $P_{\mathcal{W}}(\mathcal{S})$ en $\mathcal{H}$. Por otra parte, el subespacio $A^{1 / 2}\left(\mathcal{S}_{2}\right)=P_{\mathcal{W}}(\mathcal{S}) \oplus\{0\}$ no es cerrado ya que $\mathcal{S}+\mathcal{T}$ no es cerrado (ver la Proposición 1.1.18 ó $[11,47])$. Luego, por la Proposición 2.2.1, el par $\left(B, \mathcal{S}_{2}\right)$ no es compatible.

Lo apuntado en el ejemplo anterior nos motiva a ampliar la definición de operador cortocircuito.

Definición. Sean $B \in L(\mathcal{H})^{s}$ con descomposición polar $B=J A$ y $\mathcal{S}$ un subespacio cerrado de $\mathcal{H}$. Consideremos el subespacio $\mathcal{M}=\overline{A^{1 / 2}(\mathcal{S})}$ y supongamos que el operador $P_{J(\mathcal{M})^{\perp} / / \mathcal{M}} A^{1 / 2}$ está bien definido y es acotado. Luego, se define el operador cortocircuito de $B$ a $\mathcal{S}$ mediante

$$
B_{/ \mathcal{S}}=J A^{1 / 2} P_{J(\mathcal{M}) \perp / / \mathcal{M}} A^{1 / 2}
$$

y la compresión a $\mathcal{S}$ como $B_{\mathcal{S}}=J A^{1 / 2} P_{\mathcal{M} / / J(\mathcal{M}) \perp} A^{1 / 2}$. 
Observación 3.3.3. Recordemos que, si la composición $P_{J(\mathcal{M})^{\perp} / / \mathcal{M}} A^{1 / 2}$ está bien definida y acotada, entonces $R\left(A^{1 / 2}\right) \subset \mathcal{M}+J(\mathcal{M})^{\perp}$ y $\mathcal{M} \cap J(\mathcal{M})^{\perp}=\{0\}$. Por lo tanto, la proyección $P_{J(\mathcal{M})^{\perp} / / \mathcal{M}}$ es un operador densamente definido (posiblemente no acotado) que admite un operador adjunto.

Proposición 3.3.4. Sean $B \in L(\mathcal{H})^{s}$ con descomposición polar $B=J A$ y $\mathcal{S}$ un subespacio cerrado de $\mathcal{H}$. Supongamos que el operador $P_{J(\mathcal{M}) \perp / / \mathcal{M}} A^{1 / 2}$ está bien definido y es acotado. Luego, los operadores $B_{/ \mathcal{S}}$ y $B_{\mathcal{S}}$ dados por la Definición 3.3 son operadores autoadjuntos.

Demostración. Si $P=P_{J(\mathcal{M})^{\perp} / / \mathcal{M}}$, es fácil ver que $J P$ es un operador simétrico con dominio $\mathcal{M}+J(\mathcal{M})^{\perp}$. De hecho, si $m_{1}, m_{2} \in \mathcal{M}$ y $n_{1}, n_{2} \in J(\mathcal{M})^{\perp}$,

$$
\begin{aligned}
\left\langle J P\left(m_{1}+n_{1}\right), m_{2}+n_{2}\right\rangle & =\left\langle P\left(m_{1}+n_{1}\right), J\left(m_{2}+n_{2}\right)\right\rangle=\left\langle m_{1}, J m_{2}+J n_{2}\right\rangle=\left\langle m_{1}, J m_{2}\right\rangle= \\
& =\left\langle J m_{1}, m_{2}\right\rangle=\left\langle J m_{1}+J n_{1}, m_{2}\right\rangle=\left\langle J\left(m_{1}+n_{1}\right), P\left(m_{2}+n_{2}\right)\right\rangle= \\
& =\left\langle m_{1}+n_{1}, J P\left(m_{2}+n_{2}\right)\right\rangle .
\end{aligned}
$$

Luego, para todo $x, y \in \mathcal{H}$,

$$
\langle B / \mathcal{S} x, y\rangle=\left\langle J P A^{1 / 2} x, A^{1 / 2} y\right\rangle=\left\langle A^{1 / 2} x, J P A^{1 / 2} y\right\rangle=\langle x, B / \mathcal{S} y\rangle,
$$

ya que $R\left(A^{1 / 2}\right)$ está contenido en el dominio de $J P$. Por lo tanto, $B / \mathcal{S} \in L(\mathcal{H})^{s}$.

Proposición 3.3.5. Sea $B \in L(\mathcal{H})^{s}$ con descomposición polar $B=J A$ y supongamos que $B / \mathcal{S}$ y $B_{\mathcal{S}}$ están dados por la Definición 3.3. Luego,

1. $B(\mathcal{S}) \subseteq R\left(B_{\mathcal{S}}\right) \subseteq \overline{B(\mathcal{S})}$ y $N\left(B_{\mathcal{S}}\right)=B(\mathcal{S})^{\perp}$;

2. $R(B) \cap \mathcal{S}^{\perp} \subseteq R(B / \mathcal{S}) \subseteq R\left(A^{1 / 2}\right) \cap \mathcal{S}^{\perp}$ y $N(B / \mathcal{S})=A^{-1 / 2}(\mathcal{M})$.

Demostración. 1. Es fácil ver que $B(\mathcal{S})=J A^{1 / 2}\left(A^{1 / 2}(\mathcal{S})\right)=B_{\mathcal{S}}(\mathcal{S}) \subseteq R\left(B_{\mathcal{S}}\right) \subseteq J A^{1 / 2}(\mathcal{M}) \subseteq$ $\overline{B(\mathcal{S})}$. Por otra parte, como $\mathcal{M} \subseteq N(A)^{\perp}$, entonces

$$
N\left(B_{\mathcal{S}}\right)=N\left(P_{\mathcal{M} / / J(\mathcal{M})^{\perp}} A^{1 / 2}\right)=A^{-1 / 2}\left(J(\mathcal{M})^{\perp}\right)=B^{-1}\left(\mathcal{S}^{\perp}\right)=B(\mathcal{S})^{\perp} .
$$

2. Si $y \in R(B) \cap \mathcal{S}^{\perp}$ entonces existe $x \in \mathcal{H}$ tal que $y=B x \in \mathcal{S}^{\perp}$. Notemos que $A^{1 / 2} x \in J(\mathcal{M})^{\perp}$ y $B_{/ \mathcal{S}} x=J A^{1 / 2} P_{J(\mathcal{M})^{\perp} / / \mathcal{M}}\left(A^{1 / 2} x\right)=B x=y$. Luego, $R(B) \cap \mathcal{S}^{\perp} \subseteq R(B / \mathcal{S})$. Por otro lado, $R\left(B_{/ \mathcal{S}}\right) \subseteq J A^{1 / 2}\left(J(\mathcal{M})^{\perp}\right)=A^{1 / 2}\left(A^{-1 / 2}\left(\mathcal{S}^{\perp}\right)\right)=\mathcal{S}^{\perp} \cap R\left(A^{1 / 2}\right)$. Al igual que en el item 1, vemos que $N\left(B_{/ \mathcal{S}}\right)=N\left(P_{J(\mathcal{M})^{\perp} / / \mathcal{M}^{A}} A^{1 / 2}\right)=A^{-1 / 2}(\mathcal{M})$.

En general, las inclusiones de los items 1. y 2. de la proposición anterior son estrictas. A continuación presentamos dos ejemplos que ilustran la afirmación anterior, los mismos están inspirados en los publicados en [3] y [22].

Ejemplo 3.3.6. Sea $A \in L(\mathcal{H})^{+}$tal que $R(A)$ no es cerrado. Dado $x \in R\left(A^{1 / 2}\right) \backslash R(A)$ con $\|x\|=1$, sea $\mathcal{S}^{\perp}$ el subespacio generado por $x$. Luego, $R(A) \cap \mathcal{S}^{\perp}=\{0\}$. A continuación probaremos que $A_{/ \mathcal{S}} \neq 0$ y, por lo tanto, $R(A) \cap \mathcal{S}^{\perp}=\{0\} \subsetneq R(A / \mathcal{S})$.

Consideremos el operador $X=\langle A x, x\rangle P_{\mathcal{S}^{\perp}} \in L(\mathcal{H})^{+}$. En primer lugar, notemos que $R(X) \subseteq$ $\mathcal{S}^{\perp}$. Además, es fácil ver que $X \leq A$, ya que, dado $y \in \mathcal{H}$,

$$
\begin{aligned}
\langle(A-X) y, y\rangle & =\langle A y, y\rangle-\langle A x, x\rangle\left\langle P_{\mathcal{S}^{\perp}} y, y\right\rangle=\|y\|^{2}\langle A x, x\rangle-\langle A x, x\rangle\left\|P_{\mathcal{S}^{\perp}} y\right\|^{2}= \\
& =\left\|P_{\mathcal{S}} y\right\|^{2}\langle A x, x\rangle \geq 0 .
\end{aligned}
$$

Por lo tanto, $X \in \mathcal{M}\left(A, \mathcal{S}^{\perp}\right)$. Luego, $R\left(A_{/ \mathcal{S}}\right) \neq\{0\}$ pues $0 \neq X \leq A_{/ \mathcal{S}}$. 
Ejemplo 3.3.7. Dado un operador $T \in L(\mathcal{H})^{+}$inyectivo pero no inversible, sea

$$
A=\left(\begin{array}{cc}
T & T^{1 / 2} \\
T^{1 / 2} & 0
\end{array}\right)=\left(\begin{array}{cc}
T^{1 / 2} & 0 \\
I & 0
\end{array}\right)\left(\begin{array}{cc}
T^{1 / 2} & I \\
0 & 0
\end{array}\right) \in L(\mathcal{H} \oplus \mathcal{H})^{+}
$$

En primer lugar, notemos que $R(A)$ es cerrado ya que

$$
R\left(\left(\begin{array}{cc}
T^{1 / 2} & I \\
0 & 0
\end{array}\right)\right)=\left(R\left(T^{1 / 2}\right)+R(I)\right) \oplus\{0\}=\mathcal{H} \oplus\{0\} .
$$

Si consideramos el subespacio $\mathcal{S}=\mathcal{H} \oplus\{0\}$ de $\mathcal{H} \oplus \mathcal{H}$, es fácil ver que $A_{/ \mathcal{S}}=0$. De hecho, como $A_{/ \mathcal{S}}=\inf \left\{Q^{*} A Q: Q \in \mathcal{Q}, N(Q)=\mathcal{S}\right\}$, tenemos que

$$
0 \leq A_{/ \mathcal{S}} \leq P_{\mathcal{S}^{\perp}} A P_{\mathcal{S}^{\perp}}=\left(\begin{array}{cc}
0 & 0 \\
0 & I
\end{array}\right)\left(\begin{array}{cc}
T & T^{1 / 2} \\
T^{1 / 2} & 0
\end{array}\right)\left(\begin{array}{ll}
0 & 0 \\
0 & I
\end{array}\right)=0 .
$$

Por lo tanto, $A_{\mathcal{S}}=A$ y $A(\mathcal{S}) \subsetneq R\left(A_{\mathcal{S}}\right) \subsetneq \overline{A(\mathcal{S})}$.

Sean $A \in L(\mathcal{H})^{+}$y $\mathcal{S}$ un subespacio cerrado de $\mathcal{H}$. El rango y el núcleo de $A_{/ \mathcal{S}}$ y $A_{\mathcal{S}}$ fueron estudiados originalmente en [3], [51] y [59]. Suponiendo que el par $(A, \mathcal{S})$ es compatible, G. Corach et al. [20] obtuvieron una descripción más precisa de los mismos: $R\left(A_{\mathcal{S}}\right)=A(\mathcal{S})$, $R\left(A_{/ \mathcal{S}}\right)=R(A) \cap \mathcal{S}^{\perp}$ y $N\left(A_{/ \mathcal{S}}\right)=N(A)+\mathcal{S}$. Mas aún, estas caracterizaciones del rango y el núcleo de $A_{/ \mathcal{S}}$ y $A_{\mathcal{S}}$ se alcanzan si y sólo si el par $(A, \mathcal{S})$ es compatible. El siguiente teorema prueba que el mismo resultado es válido también para operadores autoadjuntos.

Lema 3.3.8. Sean $\mathcal{S}$ un subespacio cerrado de $\mathcal{H}$ y $B \in L(\mathcal{H})^{s}$ con descomposición polar $B=$ $J A$. Supongamos que $B_{/ \mathcal{S}}$ está dado por la Definición 3.3 y consideremos $\mathcal{M}=\overline{A^{1 / 2}(\mathcal{S})}$. Luego, valen las siguientes inclusiones:

$$
J(\mathcal{M})^{\perp} \cap R\left(A^{1 / 2}\right) \subseteq R\left(P_{J(\mathcal{M})^{\perp} / / \mathcal{M}} A^{1 / 2}\right) \subseteq J(\mathcal{M})^{\perp} \cap \overline{R\left(A^{1 / 2}\right)} .
$$

Demostración. Dada $P=P_{J(\mathcal{M})^{\perp} / / \mathcal{M}}$, supongamos que $P A^{1 / 2}$ etá bien definido y es acotado. Luego, la primera inclusión es claramente cierta. Si $y \in R\left(P A^{1 / 2}\right)$, tenemos que $y \in J(\mathcal{M})^{\perp}$ y existe $x \in \mathcal{H}$ tal que $y=P A^{1 / 2} x$. Es fácil ver que $\mathcal{M}^{\perp}+J(\mathcal{M})$ es denso en $\mathcal{H}$, está contenido en $\operatorname{dom}\left(P^{*}\right)$ y $P^{*} u=P_{\mathcal{M}^{\perp} / / J(\mathcal{M})} u$ para todo $u \in \mathcal{M}^{\perp}+J(\mathcal{M})$. Entonces, dado que $N\left(A^{1 / 2}\right) \subseteq \mathcal{M}^{\perp}$,

$$
\langle y, z\rangle=\left\langle A^{1 / 2} x, P^{*} z\right\rangle=\left\langle A^{1 / 2} x, z\right\rangle=0 \quad \text { para todo } z \in N\left(A^{1 / 2}\right) .
$$

Por lo tanto, $y \in \overline{R\left(A^{1 / 2}\right)} \cap J(\mathcal{M})^{\perp}$.

Teorema 3.3.9. Sean $B \in L(\mathcal{H})^{s}$, con descomposición polar $B=J A$, y $\mathcal{S}$ un subespacio cerrado de $\mathcal{H}$. Supongamos que $B_{/ \mathcal{S}}$ está dado por la Definición 3.3. Luego, las siguientes condiciones son equivalentes:

1. $(B, \mathcal{S})$ es compatible,

2. $R\left(B_{/ \mathcal{S}}\right)=\mathcal{S}^{\perp} \cap R(B)$ y $N\left(B_{/ \mathcal{S}}\right)=\mathcal{S}+N(B)$;

3. $R\left(B_{\mathcal{S}}\right)=B(\mathcal{S})$. 
Demostración. $1 \Rightarrow 2: \mathrm{Si}(B, \mathcal{S})$ es compatible, la ecuación $B_{/ \mathcal{S}}=B\left(I-P_{B, \mathcal{S}}\right)$ implica que $R(B / \mathcal{S}) \subseteq R(B) \cap \mathcal{S}^{\perp}$, mientras que la otra inclusión ya la hemos probado en la Proposición 3.3.5. También de la Proposición 3.3.5, sabemos que

$$
\begin{aligned}
N(B / \mathcal{S}) & =A^{-1 / 2}(\mathcal{M})=A^{-1 / 2}\left(\overline{A^{1 / 2}(\mathcal{S})}\right)=A^{-1 / 2}\left(\overline{A^{1 / 2}(\mathcal{S})} \cap R\left(A^{1 / 2}\right)\right)= \\
& =A^{-1 / 2}\left(A^{1 / 2}(\mathcal{S})\right)=\mathcal{S}+N\left(A^{1 / 2}\right)=\mathcal{S}+N(B),
\end{aligned}
$$

ya que, por la Proposición 2.2.1, $A^{1 / 2}(\mathcal{S})$ es cerrado en $R\left(A^{1 / 2}\right)$.

2 $\Rightarrow$ 3: Sea $P=P_{J(\mathcal{M})^{\perp} / / \mathcal{M}}$. Si $A^{-1 / 2}(\mathcal{M})=\mathcal{S}+N(B)=N(B / \mathcal{S})$ entonces $\mathcal{M} \cap R\left(A^{1 / 2}\right)=$ $A^{1 / 2}(\mathcal{S}+N(B))=A^{1 / 2}(\mathcal{S})$, es decir, $A^{1 / 2}(\mathcal{S})$ es cerrado en $R\left(A^{1 / 2}\right)$. Además, si $R\left(B_{/ \mathcal{S}}\right)=$ $\mathcal{S}^{\perp} \cap R(B)$, tenemos que $A^{1 / 2}\left(R\left(J P A^{1 / 2}\right)\right)=\mathcal{S}^{\perp} \cap R(A)$. Tomando la preimagen por $A^{1 / 2}$ de esta ecuación, obtenemos

$$
R\left(J P A^{1 / 2}\right)+N\left(A^{1 / 2}\right)=A^{-1 / 2}\left(\mathcal{S}^{\perp} \cap R(A)\right)=\mathcal{M}^{\perp} \cap\left(R\left(A^{1 / 2}\right)+N\left(A^{1 / 2}\right)\right)
$$

y, aplicando $J$, resulta que $R\left(P A^{1 / 2}\right)+N\left(A^{1 / 2}\right)=J(\mathcal{M})^{\perp} \cap\left(R\left(A^{1 / 2}\right)+N\left(A^{1 / 2}\right)\right)$. Como $N\left(A^{1 / 2}\right) \subseteq J(\mathcal{M})^{\perp}$, es fácil ver que

$$
R\left(P A^{1 / 2}\right)+N\left(A^{1 / 2}\right)=J(\mathcal{M})^{\perp} \cap R\left(A^{1 / 2}\right)+N\left(A^{1 / 2}\right) .
$$

Luego, por el Lema 3.3.8, concluímos que $J(\mathcal{M})^{\perp} \cap R\left(A^{1 / 2}\right)=R\left(P A^{1 / 2}\right)$. A partir de este hecho, es fácil ver que $R\left((I-P) A^{1 / 2}\right)=\mathcal{M} \cap R\left(A^{1 / 2}\right)$ y

$$
R\left(A^{1 / 2}\right)=R\left(P A^{1 / 2}\right)+R\left((I-P) A^{1 / 2}\right)=J(\mathcal{M})^{\perp} \cap R\left(A^{1 / 2}\right)+A^{1 / 2}(\mathcal{S}) .
$$

Por lo tanto, $R\left(B_{\mathcal{S}}\right)=J A^{1 / 2} P_{\mathcal{M} / / J(\mathcal{M})^{\perp}}\left(R\left(A^{1 / 2}\right)\right)=J A^{1 / 2}\left(A^{1 / 2}(\mathcal{S})\right)=B(\mathcal{S})$.

$3 \Rightarrow 1$ : La identidad $R\left(B_{\mathcal{S}}\right)=B(\mathcal{S})$ implica que

$$
\mathcal{H}=B_{\mathcal{S}}^{-1}\left(R\left(B_{\mathcal{S}}\right)\right)=B_{\mathcal{S}}^{-1}(B(\mathcal{S}))=B_{\mathcal{S}}^{-1}\left(B_{\mathcal{S}}(\mathcal{S})\right)=\mathcal{S}+N\left(B_{\mathcal{S}}\right)=\mathcal{S}+B^{-1}\left(\mathcal{S}^{\perp}\right),
$$

porque, por la Proposición 3.3.5, $N\left(B_{\mathcal{S}}\right)=B^{-1}\left(\mathcal{S}^{\perp}\right)$. Por lo tanto, $(B, \mathcal{S})$ es compatible.

Observación 3.3.10. Algunos de los resultados de la sección 4.2, enunciados para pares $(B, \mathcal{S})$ compatibles, siguen valiendo al considerar la nueva definición para el complemento de Schur. Por ejemplo, no es difícil probar que, dados $B \in L(\mathcal{H})^{s}$ y un subespacio cerrado $\mathcal{S}$ de $\mathcal{H}$ tales que $B_{/ \mathcal{S}}$ está bien definido, las siguientes condiciones son equivalentes:

1. $(B, \mathcal{S})$ es compatible y $\mathcal{S}$ es $B$-no negativo;

2. $B / \mathcal{S}=\min _{\leq}\left\{Q^{*} B Q: Q \in \mathcal{Q}, N(Q)=\mathcal{S}\right\}$;

3. $B / \mathcal{S}=\operatorname{máx}_{\leq}\left\{X \in L(\mathcal{H})^{s}: X \leq B, R(X) \subseteq \mathcal{S}^{\perp}\right\}$. 


\section{Capítulo 4}

\section{Complementos de Schur en espacios de Krein}

Sean $\mathcal{H}$ un espacio de Krein con simetría fundamental $J$ y $A \in L(\mathcal{H})$ un operador $J$ autoadjunto con la PFU. Supongamos que $A=D D^{\#}$, donde $D \in L(\mathcal{K}, \mathcal{H})$ con $N(D)=\{0\}$ y $\mathcal{K}$ es un espacio de Krein. Dado un subespacio cerrado $\mathcal{S}$ de $\mathcal{H}$, consideremos el subespacio $\mathcal{M}=\overline{D^{\#}(\mathcal{S})}$ y supongamos que $\mathcal{M}$ es un subespacio de Krein de $\mathcal{K}$.

Definición. Bajo las hipótesis anteriores, definimos el complemento de Schur de $A$ a $\mathcal{S}$ como

$$
A_{/[\mathcal{S}]}=D P_{\mathcal{M}[\perp] / / \mathcal{M}} D^{\#}
$$

y la compresión de $A$ a $\mathcal{S}$ como $A_{[\mathcal{S}]}=D P_{\mathcal{M} / / \mathcal{M}[\perp]} D^{\#}$.

En primer lugar, veamos que los operadores $A_{[\mathcal{S}]}$ y $A_{/[\mathcal{S}]}$ están bien definidos: por la PFU de $A$, si $A=D_{i} D_{i}^{\#} \operatorname{con} D_{i} \in L\left(\mathcal{K}_{i}, \mathcal{H}\right)$ y $N\left(D_{i}\right)=\{0\}$ para $\underline{i=1,2}$, existe un isomorfismo $U \in L\left(\mathcal{K}_{1}, \mathcal{K}_{2}\right)$ tal que $D_{1}=D_{2} U$. Fijados los subespacios $\mathcal{M}_{i}=\overline{D_{i}^{\#}(\mathcal{S})}$, para $i=1,2$, notemos que $\mathcal{M}_{1}$ es un subespacio de Krein de $\mathcal{K}_{1}$ si y sólo si $\mathcal{M}_{2}=U\left(\mathcal{M}_{1}\right)$ es un subespacio de Krein de $\mathcal{K}_{2}$, y en ese caso $U P_{\mathcal{M}_{1} / / \mathcal{M}_{1}^{[\perp]}} U^{\#}=P_{\mathcal{M}_{2} / / \mathcal{M}_{2}^{[\perp]}}$. Luego,

$$
D_{1} P_{\mathcal{M}_{1} / / \mathcal{M}_{1}^{[\perp]}} D_{1}^{\#}=D_{2}\left(U P_{\mathcal{M}_{1} / / \mathcal{M}_{1}^{[\perp]}} U^{\#}\right) D_{2}^{\#}=D_{2} P_{\mathcal{M}_{2} / / \mathcal{M}_{2}^{[\perp]}} D_{2}^{\#}
$$

Las siguientes son algunas propiedades que se desprenden de la definición anterior:

1. $A_{[\mathcal{S}]}, A_{/[\mathcal{S}]} \in L(\mathcal{H})$,

2. $A_{[\mathcal{S}]}, A_{/[\mathcal{S}]}$ son operadores $J_{\mathcal{H}}$-autoadjuntos,

3. $A_{[\mathcal{S}]}+A_{/[\mathcal{S}]}=A$.

La segunda afirmación es consecuencia de que las proyecciones $P_{\mathcal{M} / / \mathcal{M}[\perp]}$ y $P_{\mathcal{M}[\perp] / / \mathcal{M}}$ son $J_{\mathcal{K}^{-}}$ autoadjuntas.

A continuación, caracterizaremos el rango y el núcleo de $A_{[\mathcal{S}]}$ y $A_{/[\mathcal{S}]}$. Para esto necesitaremos el siguiente lema técnico. 
Lema 4.0.11. Sean $\mathcal{H}$ y $\mathcal{K}$ dos espacios de Krein. Si $T \in L(\mathcal{H}, \mathcal{K})$ entonces,

1. $N\left(T^{\#}\right)=R(T)^{[\perp]}$ .

2. $T^{\#}(\mathcal{S})^{[\perp]_{\mathcal{H}}}=T^{-1}\left(\mathcal{S}^{[\perp]_{\mathcal{K}}}\right)$ para todo subespacio $\mathcal{S}$ de $\mathcal{K}$.

Proposición 4.0.12. Sean $A=D D^{\#} \in L(\mathcal{H})$ un operador J-autoadjunto con la PFU y $\mathcal{S}$ un subespacio cerrado de $\mathcal{H}$ tal que $\mathcal{M}=\overline{D^{\#}(\mathcal{S})}$ es un subespacio de Krein de $\mathcal{K}$. Entonces,

1. $A(\mathcal{S}) \subseteq R\left(A_{[\mathcal{S}]}\right) \subseteq \overline{A(\mathcal{S})}$ y $N\left(A_{[\mathcal{S}]}\right)=A(\mathcal{S})^{[\perp]} ;$

2. $R(A) \cap \mathcal{S}^{[\perp]} \subseteq R\left(A_{/[\mathcal{S}]}\right) \subseteq R(D) \cap \mathcal{S}^{[\perp]} \quad$ y $\quad N\left(A_{/[\mathcal{S}]}\right)=\left(D^{\#}\right)^{-1}(\mathcal{M})$.

Demostración. 1. Notemos que,

$$
A(\mathcal{S})=D\left(D^{\#}(\mathcal{S})\right)=A_{[\mathcal{S}]}(\mathcal{S}) \subseteq R\left(A_{[\mathcal{S}]}\right) \subseteq D(\mathcal{M})=D\left(\overline{D^{\#}(\mathcal{S})}\right) \subseteq \overline{D D^{\#}(\mathcal{S})}=\overline{A(\mathcal{S})}
$$

Por otra parte, como $N(D)=\{0\}$, tenemos que

$$
N\left(A_{[\mathcal{S}]}\right)=N\left(P_{\mathcal{M} / / \mathcal{M}^{[\perp]}} D^{\#}\right)=\left(D^{\#}\right)^{-1}\left(\mathcal{M}^{[\perp]}\right)=A^{-1}\left(\mathcal{S}^{[\perp]}\right)=A(\mathcal{S})^{[\perp]} .
$$

2. En primer lugar observemos que, por la Observación $1.2 .24, R(D)$ no depende de la factorización utilizada. Ahora, si $y \in R(A) \cap \mathcal{S}^{[\perp]}$ entonces existe un vector $x \in \mathcal{H}$ tal que $y=A x \in \mathcal{S}^{[\perp]}$. Notemos que $D^{\#} x \in \mathcal{M}^{[\perp]}$ y $A_{/[\mathcal{S}]} x=D P_{\mathcal{M}[\perp] / / \mathcal{M}}\left(D^{\#} x\right)=D D^{\#} x=y$. Luego, $R(A) \cap \mathcal{S}^{[\perp]} \subseteq R\left(A_{/[\mathcal{S}]}\right)$. Por otra parte, $R\left(A_{/[\mathcal{S}]}\right) \subseteq D\left(\mathcal{M}^{[\perp]}\right)=D\left(D^{-1}\left(\mathcal{S}^{[\perp]}\right)\right)=\mathcal{S}^{[\perp]} \cap R(D)$. $\mathrm{Al}$ igual que en el item 1 , se deduce que $N\left(A_{/[\mathcal{S}]}\right)=N\left(P_{\mathcal{M}[\perp] / / \mathcal{M}} D^{\#}\right)=\left(D^{\#}\right)^{-1}(\mathcal{M})$.

En general, las inclusiones entre rangos probadas en la proposición anterior son estrictas. El próximos ejemplo, similar a uno de los presentados en el capítulo anterior, ilustra esta afirmación. De manera análoga, pueden contruirse ejemplos para mostrar que las demás inclusiones son estrictas.

Ejemplo 4.0.13. Dado un espacio de Hilbert $\mathcal{H}$, sea $T \in L(\mathcal{H})$ un operador autoadjunto tal que $N(T)=\{0\}$ y $R(T)$ no es cerrado. Si la descomposición polar de $T$ está dada por $T=U|T|$, con $|T| \in L(\mathcal{H})^{+}, U=U^{*}=U^{-1}$ y $U|T|=|T| U$, consideremos el espacio de Krein $\mathcal{H}_{1}=\mathcal{H}$ con simetría fundamental $U$. Además, sea $\mathcal{H}_{2}=\mathcal{H} \oplus \mathcal{H}$ el espacio de Krein cuya simetría fundamental $J$ está dada por

$$
J=\left(\begin{array}{cc}
I & 0 \\
0 & U
\end{array}\right)
$$

respecto a la descomposición matricial inducida por $\mathcal{H} \oplus\{0\}$. Sea $A \in L\left(\mathcal{H}_{2}\right)$ el operador $J$ autoadjunto definido por

$$
A=\left(\begin{array}{cc}
T & U|T|^{1 / 2} \\
|T|^{1 / 2} & I
\end{array}\right)
$$

y notemos que $A=D D^{\#}$, donde $D \in L\left(\mathcal{H}_{1}, \mathcal{H}_{2}\right)$ está dado por

$$
D=\left(\begin{array}{c}
U|T|^{1 / 2} \\
I
\end{array}\right)
$$

Si $\mathcal{S}=\mathcal{H} \oplus\{0\}$ entonces $D^{\#}(\mathcal{S})=R\left(|T|^{1 / 2}\right) \subsetneq \mathcal{H}_{1}$ y $\mathcal{M}=\overline{D^{\#}(\mathcal{S})}=\mathcal{H}_{1}$. Luego, $N(D)=$ $\mathcal{M}^{[\perp] \mathcal{H}_{1}}=\{0\}$, ya que $\mathcal{H}_{1}=\mathcal{M} \subseteq \overline{R\left(D^{\#}\right)}=N(D)^{[\perp]_{\mathcal{H}_{1}}}$. 
Entonces, $A=D D^{\#}$ es una descomposición como la del Teorema 1.2.21 y

$$
P_{\mathcal{M} / / \mathcal{M}[\perp]}=I \in L\left(\mathcal{H}_{1}\right) \text {. }
$$

Luego, tenemos que $A_{[\mathcal{S}]}=A$ y $A_{/[\mathcal{S}]}=0 \in L\left(\mathcal{H}_{2}\right)$. Por lo tanto,

$$
\begin{aligned}
A(\mathcal{S})=R(T) \oplus R\left(|T|^{1 / 2}\right) & \subsetneq R\left(|T|^{1 / 2}\right) \oplus \mathcal{H}=\left(R(T)+R\left(|T|^{1 / 2}\right)\right) \oplus\left(R\left(|T|^{1 / 2}\right)+\mathcal{H}\right) \\
& =R\left(A_{[\mathcal{S}]}\right) \subsetneq \mathcal{H}_{2}=\overline{A(\mathcal{S})} .
\end{aligned}
$$

Proposición 4.0.14. Dado un operador $J$-autoadjunto $A \in L(\mathcal{H})$ con la $P F U$, sea $D \in L(\mathcal{K}, \mathcal{H})$ tal que $N(D)=\{0\}$ y $A=D D^{\#}$. Sea $\mathcal{S}$ un subespacio cerrado de $\mathcal{H}$ tal que $\mathcal{M}=\overline{D^{\#}(\mathcal{S})}$ es un subespacio de Krein de $\mathcal{K}$. Si $\mathcal{T}$ es un subespacio cerrado de $\mathcal{H}$ tal que $\mathcal{S} \subseteq \mathcal{T} \subseteq\left(D^{\#}\right)^{-1}(\mathcal{M})$ entonces $\overline{D^{\#}(\mathcal{T})}=\mathcal{M} y$

$$
A_{/[\mathcal{T}]}=A_{/[\mathcal{S}]} .
$$

Demostración. Sea $\mathcal{T}$ un subespacio cerrado de $\mathcal{H}$ tal que $\mathcal{S} \subseteq \mathcal{T} \subseteq\left(D^{\#}\right)^{-1}(\mathcal{M})$. Aplicando $D^{\#}$ a estas inclusiones, tenemos que $D^{\#}(\mathcal{S}) \subseteq D^{\#}(\mathcal{T}) \subseteq D^{\#}\left(\left(D^{\#}\right)^{-1}(\mathcal{M})\right) \subseteq \mathcal{M}$. Por lo tanto, $\overline{D^{\#}(\mathcal{T})}=\mathcal{M}$ y $A_{/[\mathcal{T}]}=A_{/[\mathcal{S}]}$.

\subsection{Propiedades extremales para subespacios $J$-definidos}

El objetivo de esta sección es mostrar que, si $\mathcal{S}$ es un subespacio $J$-definido de un espacio de Krein $\mathcal{H}$, el complemento de Schur de un operador $J$-autoadjunto $A \in L(\mathcal{H})$ a $\mathcal{S}$ puede caracterizarse como un elemento extremal dentro de cierto conjunto de operadores. Más precisamente, obtendremos una representación para $A_{/[\mathcal{S}]}$ similar a la probada por W. N. Anderson Jr. y G. E. Trapp [3, Teorema 1] para el complemento de Schur de operadores positivos actuando en un espacio de Hilbert.

Los resultados de esta sección están enunciados tanto para subespacios $J$-no negativos como para subespacios $J$-no positivos, sin embargo presentaremos las demostraciones para subespacios $J$-no negativos. Las demostraciones del otro caso son análogas, realizando las modificaciones pertinentes.

Sea $A \in L(\mathcal{H})$ un operador $J$-autoadjunto con la PFU. Si $A=D D^{\#}$, siendo $D \in L(\mathcal{K}, \mathcal{H})$ con $N(D)=\{0\}$ y $\mathcal{K}$ un espacio de Krein, consideremos el conjunto

$$
\mathcal{I}(A)=\left\{X=E E^{\#}: E \in L(\mathcal{K}, \mathcal{H}), R(E) \subseteq R(D)\right\} .
$$

Por la Observación 1.2.24, el subespacio $R(D)$ sólo depende del operador $A$ y, en consecuencia, lo mismo ocurre con el conjunto $\mathcal{I}(A)$. Luego, dado un subespacio cerrado $\mathcal{S}$ de $\mathcal{H}$, consideremos dos subconjuntos de $\mathcal{I}(A)$ :

$$
\begin{aligned}
\mathcal{M}^{-}\left(A, \mathcal{S}^{[\perp]}\right) & =\left\{X \in \mathcal{I}(A): X \leq_{J} A, R(X) \subseteq \mathcal{S}^{[\perp]}\right\}, \\
\mathcal{M}^{+}\left(A, \mathcal{S}^{[\perp]}\right) & =\left\{X \in \mathcal{I}(A): A \leq_{J} X, R(X) \subseteq \mathcal{S}^{[\perp]}\right\} .
\end{aligned}
$$

El resultado principal de esta sección es el siguiente teorema:

Teorema 4.1.1. Dados un operador J-autoadjunto $A \in L(\mathcal{H})$ con la PFU y un subespacio cerrado $\mathcal{S}$ de $\mathcal{H}$, supongamos que el subespacio $\mathcal{M}=\overline{D^{\#}(\mathcal{S})}$ es un subespacio de Krein de $\mathcal{K}$, siendo $D \in L(\mathcal{K}, \mathcal{H})$ tal que $N(D)=\{0\}$ y $A=D D^{\#}$. Luego, 
1. si $\mathcal{M}$ es $J$-no negativo entonces $A_{/[\mathcal{S}]}=\operatorname{máx}_{\leq J} \mathcal{M}^{-}\left(A, \mathcal{S}^{[\perp]}\right)$.

2. si $\mathcal{M}$ es $J$-no positivo entonces $A_{/[\mathcal{S}]}=\min _{\leq J} \mathcal{M}^{+}\left(A, \mathcal{S}^{[\perp]}\right)$.

Antes de probarlo, estudiaremos un caso particular que resulta interesante en sí mismo. Si consideramos el operador identidad $I \in L(\mathcal{H})$, notemos que $I$ tiene la PFU ya que $I^{2}=I \leq_{J} I$, es decir, satisface una de las condiciones suficientes apuntadas en el Teorema 1.2.25. Además, la única factorización de $I$ (salvo isomorfismos) es la dada por $I=D D^{\#}$, con $D=I \in L(\mathcal{H})$. Luego, dado un subespacio de Krein $\mathcal{S}$, resulta que $\mathcal{M}=\mathcal{S}$ y el complemento de Schur de $I$ a $\mathcal{S}$ coincide con la proyección $J$-ortogonal con núcleo $\mathcal{S}$. Además, $\mathcal{I}(I)=L(\mathcal{H})$ y los conjuntos $\mathcal{M}^{-}\left(I, \mathcal{S}^{[\perp]}\right)$ y $\mathcal{M}^{+}\left(I, \mathcal{S}^{[\perp]}\right)$ son naturalmente comparables con el del Teorema 3.1.1.

El próximo lema describe las condiciones de extremalidad que tienen las proyecciones $J$ autoadjuntas con núcleo $J$-definido, probando el Teorema 4.1.1 en el caso particular del operador identidad.

Lema 4.1.2. Sean $\mathcal{S}$ un subespacio de Krein de $\mathcal{H}$ y $Q=P_{\mathcal{S}^{[\perp]} / / \mathcal{S}}$ la proyección J-autoadjunta con núcleo $\mathcal{S}$. Luego,

1. si $\mathcal{S}$ es $J$-no negativo entonces $Q=\operatorname{máx}_{\leq J} \mathcal{M}^{-}\left(I, \mathcal{S}^{[\perp]}\right)$.

2. si $\mathcal{S}$ es $J$-no positivo entonces $Q=\min _{\leq} \mathcal{M}^{+}\left(I, \mathcal{S}^{[\perp]}\right)$.

Demostración. Supongamos que $\mathcal{S}$ es un subespacio (de Krein) $J$-no negativo de $\mathcal{H}$. Luego, por la Proposición 2.3.7, tenemos que $Q$ es $J$-contractiva y $R(Q)=\mathcal{S}^{[\perp]}$. Por lo tanto, $Q \in \mathcal{M}^{-}\left(I, \mathcal{S}^{[\perp]}\right)$.

Además, si $X \in \mathcal{M}^{-}\left(I, \mathcal{S}^{[\perp]}\right)$ entonces $X \leq{ }_{J} Q$ : de hecho, la inclusión $R(X) \subseteq \mathcal{S}^{[\perp]}$ implica que $Q X=X$, y luego $Q X Q=(Q X) Q=X Q=Q X=X$ porque $X$ y $Q$ son $J$-autoadjuntos. Entonces, si $x \in \mathcal{H}$,

$$
[(Q-X) x, x]=[Q(I-X) Q x, x]=[(I-X) Q x, Q x] \geq 0,
$$

i.e. $X \leq_{J} Q$. Por lo tanto, $Q=\operatorname{máx}_{\leq J} \mathcal{M}^{-}\left(I, \mathcal{S}^{[\perp]}\right)$.

Ahora sí, presentamos la demostración del Teorema 4.1.1.

Demostración del Teorema 4.1.1. Sea $Q=P_{\mathcal{M}}^{[\perp] / / \mathcal{M}}$ y supongamos que $\mathcal{M}$ es $J$-no negativo (i.e. $Q$ es $J$-contractiva). Notemos que $A_{/[\mathcal{S}]}=(D Q)(D Q)^{\#}$ y $R(D Q) \subseteq R(D)$, por lo que $A_{/[\mathcal{S}]} \in \mathcal{I}(A)$. Como $Q \leq_{J} I$ tenemos que $A_{/[\mathcal{S}]}=D Q D^{\#} \leq_{J} D D^{\#}=A$ y, por la Proposición 4.0.12, $R\left(A_{/[\mathcal{S}]}\right) \subseteq \mathcal{S}^{[\perp]}$. Entonces, $A_{/[\mathcal{S}]} \in \mathcal{M}^{-}\left(A, \mathcal{S}^{[\perp]}\right)$.

Además, $A_{/[\mathcal{S}]}=\operatorname{máx}_{\leq} \mathcal{M}^{-}\left(A, \mathcal{S}^{[\perp]}\right)$. De hecho, si $X=E E^{\#} \in \mathcal{M}^{-}\left(A, \mathcal{S}^{[\perp]}\right)$ entonces $R(E) \subseteq$ $R(D)$ y, por el Teorema de Douglas 1.1.1, la ecuación $D Y=E$ admite una solución en $L(\mathcal{K})$. Si $Z \in L(\mathcal{K})$ es una solución de la ecuación anterior, tenemos que $X=D Z Z^{\#} D^{\#}$. Como $X \leq_{J} A$, dado un vector $x \in \mathcal{H}$,

$$
\left[\left(I_{\mathcal{K}}-Z Z^{\#}\right) D^{\#} x, D^{\#} x\right]_{\mathcal{K}}=\left[D\left(I-Z Z^{\#}\right) D^{\#} x, x\right]_{\mathcal{H}}=[(A-X) x, x]_{\mathcal{H}} \geq 0,
$$

entonces $\left[\left(I_{\mathcal{K}}-Z Z^{\#}\right) y, y\right]_{\mathcal{K}} \geq 0$ para todo $y \in \overline{R\left(D^{\#}\right)}=N(D)^{[\perp]_{\mathcal{K}}}=\mathcal{K}$. Luego, $Z Z^{\#} \leq_{J} I_{\mathcal{K}}$. Dado que $R(X) \subseteq \mathcal{S}^{[\perp]}$, podemos concluir que $R\left(Z Z^{\#} D^{\#}\right) \subseteq D^{-1}\left(\mathcal{S}^{[\perp]}\right)=\mathcal{M}^{[\perp]}$ y luego 
$R\left(Z Z^{\#}\right)=Z Z^{\#}\left(\overline{R\left(D^{\#}\right)}\right) \subseteq \overline{R\left(Z Z^{\#} D^{\#}\right)} \subseteq \mathcal{M}^{[\perp]}$. Entonces, $Z Z^{\#} \in \mathcal{M}^{-}\left(I, \mathcal{M}^{[\perp]}\right)$ y, poe el Lema 4.1.2, $Z Z^{\#} \leq_{J} Q$ (notemos que el espacio de Krein considerado en este argumento es $\mathcal{K}$ ). Por lo tanto,

$$
X=D Z Z^{\#} D^{\#} \leq_{J} D Q D^{\#}=A_{/[\mathcal{S}]},
$$

i.e. $A_{/[\mathcal{S}]}=\operatorname{máx}_{\leq J} \mathcal{M}^{-}\left(A, \mathcal{S}^{[\perp]}\right)$.

Corolario 4.1.3. Sea $\mathcal{S}$ un subespacio de Krein de $\mathcal{H}$. Si $Q=P_{\mathcal{S}^{[\perp]} / / \mathcal{S}}$, existen dos subespacios de Krein $\mathcal{S}_{+}$y $\mathcal{S}_{-}$de $\mathcal{H}$ tales que $\mathcal{S}=\mathcal{S}_{+}+\dot{\mathcal{S}} \mathcal{S}_{-} y$

$$
Q=\operatorname{máx}_{\leq J} \mathcal{M}^{-}\left(I, \mathcal{S}_{+}^{[\perp]}\right) \operatorname{mín}_{\leq J} \mathcal{M}^{+}\left(I, \mathcal{S}_{-}^{[\perp]}\right) .
$$

Demostración. Si $\mathcal{S}$ es un subespacio de Krein de $\mathcal{H}$ entonces, por el Teorema 2.3.9, $Q=Q_{+} Q_{-}$, siendo $Q_{+}$una proyección $J$-contractiva y $Q_{-}$una proyección que conmutan entre sí. También podemos probar que $\left(I-Q_{+}\right)\left(I-Q_{-}\right)=0$ (ver la demostración del Teorema 2.3.9) por lo que $I-Q=\left(I-Q_{+}\right)+\left(I-Q_{-}\right)$y $\mathcal{S}=N(Q)=N\left(Q_{+}\right) \dot{+} N\left(Q_{-}\right)$.

Por el Lema 4.1.2, sabemos que $Q_{+}=\operatorname{máx}_{\leq_{J}} \mathcal{M}^{-}\left(I, R\left(Q_{+}\right)\right)$y $Q_{-}=\operatorname{mín}_{\leq_{J}} \mathcal{M}^{+}\left(I, R\left(Q_{-}\right)\right)$. Entonces, considerando los subespacios $\mathcal{S}_{ \pm}=N\left(Q_{ \pm}\right)$, completamos la demostración.

Corolario 4.1.4. Sean $\mathcal{H}$ un espacio de Krein y $A \in L(\mathcal{H})$ un operador $J$-autoadjunto con la PFU. Consideremos una factorización $A=D D^{\#}$, donde $D \in L(\mathcal{K}, \mathcal{H})$ con $N(D)=\{0\}$ y $\mathcal{K}$ es un espacio de Krein. Si $A$ tiene rango cerrado y $\mathcal{S}$ es un subespacio cerrado de $\mathcal{H}$ tal que $\mathcal{M}=\overline{D^{\#}(\mathcal{S})}$ es un subespacio de Krein de $\mathcal{K}$, entonces existen dos subespacios cerrados $\mathcal{S}_{+}$y $\mathcal{S}_{-}$de $\mathcal{H}$ tales que $\mathcal{S}_{+} \dot{+} \mathcal{S}_{-}=\left(D^{\#}\right)^{-1}(\mathcal{M})$ y

$$
A_{/[\mathcal{S}]}=\operatorname{máx}_{\leq J} \mathcal{M}^{-}\left(A, \mathcal{S}_{+}^{[\perp]}\right)+\operatorname{mín}_{\leq J} \mathcal{M}^{+}\left(A, \mathcal{S}_{-}^{[\perp]}\right)-A .
$$

Demostración. Supongamos que $\mathcal{M}$ es un subespacio de Krein de $\mathcal{K}$ y sea $Q=P_{\mathcal{M}}[\perp] / / \mathcal{M}$. Por el Teorema 2.3.9, existen dos proyecciones $Q_{+}$y $Q_{-}$que conmutan entre sí tales que $Q=Q_{+} Q_{-}$, $Q_{+}$es $J$-contractiva, $Q_{-}$es $J$-expansiva y $N(Q)=N\left(Q_{+}\right) \dot{+} N\left(Q_{-}\right)$(esta última afirmación se desprende de la demostración del Teorema 2.3.9).

Sea $\mathcal{S}_{ \pm}=\left(D^{\#}\right)^{-1}\left(N\left(Q_{ \pm}\right)\right)$y definamos $\mathcal{M}_{ \pm}=\overline{D^{\#}\left(\mathcal{S}_{ \pm}\right)}$. Por la Observación 1.2.24, $R\left(D^{\#}\right)=$ $\mathcal{K}$ y en consecuencia $\mathcal{M}_{ \pm}=\overline{D^{\#}\left(\mathcal{S}_{ \pm}\right)}=\overline{N\left(Q_{ \pm}\right) \cap R\left(D^{\#}\right)}=N\left(Q_{ \pm}\right)$. Por lo tanto, $A_{/\left[\mathcal{S}_{ \pm}\right]}=$ $D Q_{ \pm} D^{\#} \mathrm{y}$

$$
A_{[\mathcal{S}]}=D(I-Q) D^{\#}=D\left(\left(I-Q_{+}\right)+\left(I-Q_{-}\right)\right) D^{\#}=A_{\left[\mathcal{S}_{+}\right]}+A_{\left[\mathcal{S}_{-}\right]} .
$$

Además, recordemos que, por la Proposición 2.3.7, los subespacios $\mathcal{M}_{+}$y $\mathcal{M}_{-}$son $J$-no negativo y $J$-no positivo, respectivamente. Luego, utilizando las caracterizaciones del Teorema 4.1.1, tenemos que

$$
\begin{aligned}
A_{/[\mathcal{S}]} & =A-A_{[\mathcal{S}]}=A-\left(A_{\left[\mathcal{S}_{+}\right]}+A_{\left[\mathcal{S}_{-}\right]}\right)=A_{/\left[\mathcal{S}_{+}\right]}+A_{/\left[\mathcal{S}_{-}\right]}-A= \\
& =\operatorname{máx}_{\leq_{J}} \mathcal{M}^{-}\left(A, \mathcal{S}_{+}^{[\perp]}\right)+\operatorname{minn}_{\leq_{J}} \mathcal{M}^{+}\left(A, \mathcal{S}_{-}^{[\perp]}\right)-A .
\end{aligned}
$$


Teorema 4.1.5. Sea $\mathcal{S}$ un subespacio cerrado de $\mathcal{H}$. Supongamos que $A \in L(\mathcal{H})$ es $J$-autoadjunto y satisface la PFU. Si $A=D D^{\#}$ con $D \in L(\mathcal{K}, \mathcal{H}), N(D)=\{0\}$, supongamos que $\mathcal{M}=\overline{D^{\#}(\mathcal{S})}$ es un subespacio de Krein de $\mathcal{K}$. Luego,

1. si $\mathcal{M}$ es J-no negativo entonces $A_{/[\mathcal{S}]}=\inf _{\leq J}\left\{Q^{\#} A Q: Q \in \mathcal{Q}(\mathcal{H}), N(Q)=\mathcal{S}\right\}$.

2. si $\mathcal{M}$ es $J$-no positivo entonces $A_{/[\mathcal{S}]}=\sup _{\leq_{J}}\left\{Q^{\#} A Q: Q \in \mathcal{Q}(\mathcal{H}), N(Q)=\mathcal{S}\right\}$.

Demostración. Supongamos que $\mathcal{M}$ es un subespacio $J$-no negativo y consideremos la proyección $P=P_{\mathcal{M}^{[\perp]} / / \mathcal{M}}$. Notemos que, para todo $x \in \mathcal{K}$,

$$
[P x, P x]_{\mathcal{K}}=\min _{m \in \mathcal{M}}[x-m, x-m]_{\mathcal{K}} .
$$

De hecho,dados $x \in \mathcal{K}$ y $m \in \mathcal{M}$,

$$
\begin{aligned}
{[x-m, x-m] } & =[P x+(I-P) x-m, P x+(I-P) x-m] \\
& =[P x, P x]+[(I-P) x-m,(I-P) x-m] \geq[P x, P x] .
\end{aligned}
$$

Además, $R\left(D^{\#}\right)$ es denso en $\mathcal{K}$ pues $N(D)=\{0\}$. Entonces, si $y \in \mathcal{H}$,

$$
\begin{aligned}
{\left[A_{/[\mathcal{S}]} y, y\right]_{\mathcal{H}} } & =\left[P D^{\#} y, P D^{\#} y\right]_{\mathcal{K}}=\min _{m \in \mathcal{M}}\left[D^{\#} y-m, D^{\#} y-m\right]_{\mathcal{K}}= \\
& =\inf _{s \in \mathcal{S}}\left[D^{\#}(y-s), D^{\#}(y-s)\right]_{\mathcal{K}}=\inf _{s \in \mathcal{S}}[A(y-s), y-s]_{\mathcal{H}} .
\end{aligned}
$$

Si $Q \in \mathcal{Q}(\mathcal{H})$ con $N(Q)=\mathcal{S}$, dado $x \in \mathcal{H}$,

$$
\left[Q^{\#} A Q x, x\right]_{\mathcal{H}}=[A Q x, Q x]_{\mathcal{H}}=[A(x-(I-Q) x), x-(I-Q) x]_{\mathcal{H}} \geq\left[A_{/[\mathcal{S}]} x, x\right]_{\mathcal{H}}
$$

porque $(I-Q) x \in \mathcal{S}$. Luego, $A_{/[\mathcal{S}]} \leq_{J} Q^{\#} A Q$ para toda $Q \in \mathcal{Q}(\mathcal{H})$ con $N(Q)=\mathcal{S}$ i.e. $A_{/[\mathcal{S}]}$ es una cota inferior del conjunto $\left\{Q^{\#} A Q: Q \in \mathcal{Q}(\mathcal{H}), N(Q)=\mathcal{S}\right\}$.

Dada $C$ una cota inferior cualquiera del conjunto $\left\{Q^{\#} A Q: Q \in \mathcal{Q}(\mathcal{H}), N(Q)=\mathcal{S}\right\}$, mostraremos que $C \leq_{J} A_{/[\mathcal{S}]}$. Fijemos un vector $x \in \mathcal{H}$. Si $x \notin \mathcal{S}$, notemos que para todo $s \in \mathcal{S}$ existe una proyección $Q \in \mathcal{Q}$ con $N(Q)=\mathcal{S}$ tal que $(I-Q) x=s$. Entonces,

$$
[A(x-s), x-s]_{\mathcal{H}}=[A Q x, Q x]_{\mathcal{H}} \geq[C x, x]_{\mathcal{H}}
$$

para todo $s \in \mathcal{S}$. Luego, $\left[A_{/[\mathcal{S}]} x, x\right]_{\mathcal{H}} \geq[C x, x]_{\mathcal{H}}$. Por otra parte, si $x \in \mathcal{S}$ entonces $Q^{\#} A Q x=0$ para toda $Q \in \mathcal{Q}(\mathcal{H})$ con $N(Q)=\mathcal{S}$, y tenemos que,

$$
[C x, x]_{\mathcal{H}} \leq\left[Q^{\#} A Q x, x\right]_{\mathcal{H}}=0 .
$$

Pero también $A_{/[\mathcal{S}]} x=D P_{\mathcal{M}[\perp] / / \mathcal{M}} D^{\#} x=0$ porque $D^{\#} x \in \mathcal{M}$. Entonces, $\left[A_{/[\mathcal{S}]} x, x\right]_{\mathcal{H}}=0 \geq$ $[C x, x]_{\mathcal{H}}$. Como el vector $x \in \mathcal{H}$ era arbitrario, concluímos que $A_{/[\mathcal{S}]} \geq_{J} C$ y, por lo tanto,

$$
A_{/[\mathcal{S}]}=\inf _{\leq}\left\{Q^{\#} A Q: Q \in \mathcal{Q}(\mathcal{H}), N(Q)=\mathcal{S}\right\}
$$




\subsection{Complementos de Schur de operadores $J$-positivos en es- pacios de Krein}

En el Teorema 1.2.25 de los Preliminares mencionamos que los operadores $J$-positivos tienen la propiedad de factorización única. Además, observamos que, dada una factorización $A=D D^{\#}$ como la del Teorema 1.2.21, podemos elegir el espacio vectorial $\mathcal{K}$ (que cumple el rol de dominio del factor $D$ ) de manera que resulte un espacio de Hilbert.

Dado un operador (acotado) $J$-positivo $A$ actuando en un espacio de Krein $\mathcal{H}$, en esta sección utilizaremos la siguiente factorización de $A$ : si $|A|=J A \in L(|\mathcal{H}|)^{+}$, consideremos el espacio de Hilbert $\mathcal{K}=J\left(N(A)^{\perp}\right)$ y el operador $D=\left.J|A|^{1 / 2} J\right|_{\mathcal{K}} \in L(\mathcal{K}, \mathcal{H})$. Luego, $N(D)=\{0\}$, $D^{\#}=J|A|^{1 / 2} \in L(\mathcal{H}, \mathcal{K})$ y $D D^{\#}=A$.

Notemos que, si $\mathcal{K}$ es un espacio de Hilbert y $\mathcal{S}$ es cualquier subespacio cerrado de $\mathcal{H}$, el subespacio $\mathcal{M}=\overline{D^{\#}(\mathcal{S})}$ es un subespacio cerrado de $\mathcal{K}$ y por lo tanto resulta un "subespacio de Krein" de $\mathcal{K}$. Entonces, el complemento de Schur $A_{/[\mathcal{S}]}$ está bien definido para todo subespacio cerrado $\mathcal{S}$ de $\mathcal{H}$ y además,

$$
\begin{aligned}
A_{[\mathcal{S}]} & =D P_{\mathcal{M}^{\perp}} D^{\#}=\left(J|A|^{1 / 2} J\right) P_{\mathcal{M}^{\perp}}\left(J|A|^{1 / 2}\right)=J|A|^{1 / 2}\left(J P_{\mathcal{M}^{\perp}} J\right)|A|^{1 / 2}= \\
& =J|A|^{1 / 2} P_{J\left(\mathcal{M}^{\perp}\right)}|A|^{1 / 2},
\end{aligned}
$$

donde $P_{J\left(\mathcal{M}^{\perp}\right)} \in L(\mathcal{K})$ es la proyección ortogonal sobre $J\left(\mathcal{M}^{\perp}\right)$. Esto implica que $A_{/[\mathcal{S}]}$ es un operador $J$-positivo y además si consideramos el operador $E \in L\left(\mathcal{M}^{\perp}, \mathcal{H}\right)$ definido por $E x=D x=J|A|^{1 / 2} J x$, para $x \in \mathcal{M}^{\perp}$, tenemos que

$$
A_{/[\mathcal{S}]}=E E^{\#}, \quad \text { y } \quad N(E)=\{0\},
$$

es decir, esta es la factorización única (salvo isomorfismos) de $A_{/[\mathcal{S}]}$.

Observación 4.2.1. Es importante destacar que $J\left(\mathcal{M}^{\perp}\right)={\overline{J D^{\#}(\mathcal{S})}}^{\perp}=\left(|A|^{1 / 2}(\mathcal{S})\right)^{\perp}$. Entonces, de la Ec. (4.2.1) y el item 1. del Teorema 3.1.5, podemos concluir que, si $A \in L(\mathcal{H})$ es $J$-positivo entonces

$$
A_{/[\mathcal{S}]}=J\left(|A|_{/ \mathcal{S}}\right)
$$

donde $|A|_{/ \mathcal{S}}$ es el operador cortocircuito (en el sentido de operadores actuando sobre un espacio de Hilbert) de $|A|$ a $\mathcal{S}$.

En consecuencia, el complemento de Schur de un operador $J$-positivo $A$, actuando en un espacio de Krein $\mathcal{H}$, está intimamente relacionado con el complemento de Schur del operador positivo $J A$, el cual actúa en el espacio de Hilbert $|\mathcal{H}|$. Las siguientes proposiciones traducen algunos resultados clásicos de operadores cortocircuito al contexto de un espacio de Krein. En primer lugar, enunciaremos una versión del Teorema de Douglas para operadores $J$-positivos en espacios de Krein.

Teorema 4.2.2. Sea $\mathcal{H}$ un espacio de Krein y consideremos operadores $J$-positivos $A, B \in L(\mathcal{H})$. Si $A=D D^{\#}, D \in L\left(\mathcal{K}_{1}, \mathcal{H}\right), N(D)=\{0\}$ es una factorización cualquiera de $A$ como la del Teorema 1.2.21 (resp. $B=E E^{\#}, E \in L\left(\mathcal{K}_{2}, \mathcal{H}\right), N(E)=\{0\}$ ) entonces las siguientes condiciones son equivalentes:

1. la ecuación $D X=E$ admite una solución en $L\left(\mathcal{K}_{2}, \mathcal{K}_{1}\right)$; 
2. $R(E) \subseteq R(D)$;

3. existe una constante $\lambda>0$ tal que $B \leq_{J} \lambda A$.

En este caso, existe un único $X \in L\left(\mathcal{K}_{2}, \mathcal{K}_{1}\right)$ tal que $D X=E$. Además, $N(X)=N(E)$ y

$$
\|X\|=\inf \left\{\lambda>0: B \leq_{J} \lambda A\right\} .
$$

Demostración. Recordemos que si $A$ (resp. $B$ ) es $J$-positivo entonces $\mathcal{K}_{1}$ (resp. $\mathcal{K}_{2}$ ) es un espacio de Hilbert. Luego, $D^{\#}=D^{*} J$ y $E^{\#}=E^{*} J$. Por lo tanto, la desigualdad $A \leq_{J} \lambda B$ es equivalente a $D D^{*} \leq \lambda E E^{*}$ y el resultado es consecuencia del Teorema de Douglas.

Proposición 4.2.3. Si $\mathcal{S}$ y $\mathcal{T}$ son subespacios cerrados de $\mathcal{H}$ y $A, B \in L(\mathcal{H})$ son J-positivos, luego

1. $A_{/[\mathcal{S}]}=\operatorname{máx}_{\leq J} \mathcal{M}^{-}\left(A, \mathcal{S}^{[\perp]}\right)=\operatorname{máx}_{\leq J}\left\{X \in L(\mathcal{H}): 0 \leq_{J} X \leq_{J} A, R(X) \subseteq \mathcal{S}^{[\perp]}\right\}$

2. $A_{/[\mathcal{S}]}=\inf _{\leq J}\left\{Q^{\#} A Q: Q \in \mathcal{Q}(\mathcal{H}), N(Q)=\mathcal{S}\right\}$;

3. si $A \leq_{J} B$ entonces $A_{/[\mathcal{S}]} \leq{ }_{J} B /[\mathcal{S}]$;

4. si $\mathcal{T} \subseteq \mathcal{S}$ entonces $A_{/[\mathcal{S}]} \leq_{J} A_{/[\mathcal{T}]}$.

Demostración. 1. Dados un operador $J$-positivo $A \in L(\mathcal{H})$ y un subespacio cerrado $\mathcal{S}$ de $\mathcal{H}$, $A_{/[\mathcal{S}]}=\operatorname{máx}_{\leq J} \mathcal{M}^{-}\left(A, \mathcal{S}^{[\perp]}\right)$ por el Teorema 4.1.1 (recordemos que $\mathcal{K}$ es un espacio de Hilbert). Además,

$$
\mathcal{M}^{-}\left(A, \mathcal{S}^{[\perp]}\right)=\left\{X \in L(\mathcal{H}): 0 \leq_{J} X \leq_{J} A, R(X) \subseteq \mathcal{S}^{[\perp]}\right\}
$$

Sea $\mathcal{A}=\left\{X \in L(\mathcal{H}): 0 \leq_{J} X \leq_{J} A, R(X) \subseteq \mathcal{S}^{[\perp]}\right\}$. Si $X \in \mathcal{A}$ entonces $X \geq_{J} 0$ y admite una factorization $X=E E^{\#}$, siendo $E \in L\left(\mathcal{K}_{1}, \mathcal{H}\right)$ con $N(E)=\{0\}$ y $\mathcal{K}_{1}$ un espacio de Hilbert. Luego, mediante un isomorfismo, podemos sustituir $\mathcal{K}_{1}$ por el espacio de Hilbert $\mathcal{K}$ que aparece en la descomposición de $A$. Por el Teorema 4.2.2, $R(E) \subseteq R(D)$ ya que $X \leq_{J} A$. Entonces $X \in \mathcal{I}(A)$, y las condiciones $X \leq_{J} A$ y $R(X) \subseteq \mathcal{S}^{[\perp]}$ implican que $X \in \mathcal{M}^{-}\left(A, \mathcal{S}^{[\perp]}\right)$.

Recíprocamente, si $X \in \mathcal{M}^{-}\left(A, \mathcal{S}^{[\perp]}\right)$, existe un operador $E \in L(\mathcal{K}, \mathcal{H})$ tal que $X=E E^{\#}=$ $E E^{*} J$, donde la última igualdad está justificada porque $\mathcal{K}$ es un espacio de Hilbert. Luego, $X \geq_{J} 0$ y las demás condiciones sobre $X$ aseguran que $X \in \mathcal{A}$. Entonces, $\mathcal{M}^{-}\left(A, \mathcal{S}^{[\perp]}\right) \subseteq \mathcal{A}$.

3. Si $A \leq_{J} B$ entonces $|A|=J A \leq J B=|B|$. Por el Teorema 3.1.5, $|A|_{/ \mathcal{S}} \leq|B|_{/ \mathcal{S}}$ y luego $A_{/[\mathcal{S}]}=J\left(|A|_{/ \mathcal{S}}\right) \leq_{J} J\left(|B|_{/ \mathcal{S}}\right)=B_{/[\mathcal{S}]}($ see Eq. $(4.2 .2))$.

Los items 2. y 4. también se demuestran a partir de la identidad (4.2.2).

La siguiente Proposición generaliza el item 3. del Teorema 3.1.5:

Proposición 4.2.4. Sean $\mathcal{S}$ un subespacio cerrado de $\mathcal{H}$ y $A \in L(\mathcal{H})$ un operador J-positivo. Si $A=D D^{\#}($ con $\mathcal{K}$ un espacio de Hilbert y $D \in L(\mathcal{K}, \mathcal{H})$ tal que $N(D)=\{0\})$ y $A_{/[\mathcal{S}]}=E E^{\#}$ (con $\mathcal{E}$ un espacio de Hilbert y $E \in L(\mathcal{E}, \mathcal{H})$ tal que $N(E)=\{0\}$ ), entonces

$$
R(E)=R(D) \cap \mathcal{S}^{[\perp]} .
$$


Demostración. Si $A=D D^{\#}$, siendo $D \in L(\mathcal{K}, \mathcal{H})$ con $N(D)=\{0\}$, entonces $A_{/[\mathcal{S}]}=F F^{\#}$ donde $F \in L\left(\mathcal{M}^{\perp}, \mathcal{H}\right)$ es el operador definido por $F x=D x$ para $x \in \mathcal{M}^{\perp}$. Luego,

$$
R(F)=R\left(D P_{\mathcal{M}^{\perp}}\right)=D\left(\mathcal{M}^{\perp}\right)=D\left(D^{-1}\left(\mathcal{S}^{[\perp]}\right)\right)=R(D) \cap \mathcal{S}^{[\perp]},
$$

y, por la Observación 1.2.24, $R(E)=R(F)=R(D) \cap \mathcal{S}^{[\perp]}$.

Proposición 4.2.5. Sean $\mathcal{H}$ un espacio de Krein and $A \in L(\mathcal{H})$ un operador J-positivo. Si $\mathcal{S}_{1}$ y $\mathcal{S}_{2}$ son subespacios cerrados de $\mathcal{H}$ tales que $\mathcal{S}_{1}+\mathcal{S}_{2}$ es cerrada, entonces

$$
A_{/\left[\mathcal{S}_{1}+\mathcal{S}_{2}\right]}=\left(A_{/\left[\mathcal{S}_{1}\right]}\right)_{/\left[\mathcal{S}_{2}\right]}=\left(A_{/\left[\mathcal{S}_{2}\right]}\right)_{/\left[\mathcal{S}_{1}\right]} .
$$

Demostración. Supongamos que $\mathcal{S}_{1} \operatorname{Si} \mathcal{S}_{1}$ y $\mathcal{S}_{2}$ son subespacios cerrados de $\mathcal{H}$ tales que $\mathcal{S}_{1}+\mathcal{S}_{2}$ es cerrada. Consideremos el operador $|A|=J A \in L(|\mathcal{H}|)^{+}$. Luego, por el item 4. del Teorema 3.1.5, tenemos que $|A|_{/ \mathcal{S}_{1}+\mathcal{S}_{2}}=\left(|A|_{/ \mathcal{S}_{1}}\right)_{/ \mathcal{S}_{2}}=\left(|A|_{/ \mathcal{S}_{2}}\right)_{/ \mathcal{S}_{1}}$. Entonces, por la Ec. (4.2.2),

$$
A_{/\left[\mathcal{S}_{1}+\mathcal{S}_{2}\right]}=J\left(|A|_{/ \mathcal{S}_{1}+\mathcal{S}_{2}}\right)=J\left[\left(|A|_{/ \mathcal{S}_{1}}\right)_{/ \mathcal{S}_{2}}\right]=\left(J\left(|A|_{/ \mathcal{S}_{1}}\right)\right)_{/\left[\mathcal{S}_{2}\right]}=\left(A_{/\left[\mathcal{S}_{1}\right]}\right)_{/\left[\mathcal{S}_{2}\right]} .
$$

Análogamente, $A_{/\left[\mathcal{S}_{1}+\mathcal{S}_{2}\right]}=\left(A_{/\left[\mathcal{S}_{2}\right]}\right) /\left[\mathcal{S}_{1}\right]$.

En los próximos párrafos, dada una sucesión $\left(T_{n}\right)_{n \in \mathbb{N}}$ de operadores (acotados) $J$-positivos, usaremos la notación $T_{n} \stackrel{\text { J-SOT }}{\searrow} T$ para expresar que $T_{n} \underset{n \rightarrow \infty}{\stackrel{\text { sOT }}{\longrightarrow}} T$ y que $T_{n} \geq_{J} T_{n+1}\left(\geq_{J} T\right)$ para todo $n \in \mathbb{N}$.

Notemos que $T_{n} \searrow^{\text {J-SOT }} T$ si y sólo si $J T_{n} \searrow^{\text {SOT }} J T$ : De hecho, si $T_{n} \searrow^{\text {J-SOT }} T$, tenemos que $T_{n} \underset{n \rightarrow \infty}{\stackrel{\text { SOT }}{\longrightarrow}} T$ y $T_{n} \geq_{J} T_{n+1}\left(\geq_{J} T\right)$. Equivalentemente, $J T_{n} \underset{n \rightarrow \infty}{\stackrel{\text { SOT }}{\longrightarrow}} J T$ (ya que $J$ es inversible) y $J T_{n} \geq J T_{n+1}(\geq J T)$, i. e. $J T_{n} \searrow^{\text {sot }} J T$.

La siguiente Proposición puede deducirse fácilmente utilizando la observación anterior y las Proposiciones 3.1.6 y 3.1.7.

Proposición 4.2.6. Sea $\mathcal{H}$ un espacio de Krein.

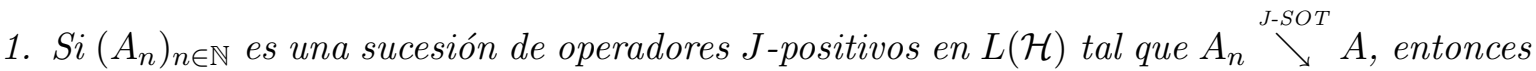

$$
A_{n /[\mathcal{S}]} \searrow^{J-S O T} A_{/[\mathcal{S}]} .
$$

2. Si $\left(\mathcal{S}_{n}\right)_{n \in \mathbb{N}}$ y $\mathcal{S}$ son subespacios cerrados de $\mathcal{H}$ tales que $\mathcal{S}_{n} \subseteq \mathcal{S}_{n+1}$ para todo $n \in \mathbb{N}$ y $\mathcal{S}=\overline{\bigcup_{n \in \mathbb{N}} \mathcal{S}_{n}}$, entonces $A_{/\left[\mathcal{S}_{n}\right]} \searrow^{J-S O T} A_{/[\mathcal{S}]}$ para todo operador $J$-positivo $A \in L(\mathcal{H})$.

Observación 4.2.7. El ejemplo 3.1.8 puede modificarse para probar que el item 2. de la Proposición 4.2.6 no es cierto para sucesiones de subespacios decrecientes, es decir, si $\mathcal{S}_{n} \supseteq \mathcal{S}_{n+1}$ para todo $n \in \mathbb{N}$ y $\mathcal{S}=\bigcap_{n \in \mathbb{N}} \mathcal{S}_{n}$. 


\section{Capítulo 5}

\section{Cuadrados mínimos indefinidos}

Un problema clásico estudiado en procesamiento de señales es la estimación de cuadrados mínimos medios de procesos estocásticos. Suponiendo que los procesos son estacionarios, este problema ha sido estudiado desde 1940 por A. N. Kolmogorov [50] y por N. Wiener [71], quien introdujo esta idea en distintas areas de la ingeniería, como la teoría de control y el procesamiento de señales. Posteriormente, a finales de los años '50, R. E. Kalman [48] extendió esta teoría a procesos no estacionarios y, desde entonces, el fitro de Kalman se transformó en una de las técnicas más útiles en aplicaciones de control. Después del trabajo de Kalman se han desarrollado teorías similares en control óptimo, conocidas como control gaussiano lineal-cuadrático (LQG). En la teoría de control LQG, las propiedades estadísticas de los procesos estocásticos subyacentes se suponen conocidos, una importante limitación en algunas aplicaciones relacionadas con la ingeniería. A comienzos de los años '80, la teoría de control $\mathcal{H}^{\infty}$ apareció como una alternativa para solucionar esta limitación en problemas prácticos (para más detalles sobre el tema, referimos al lector al trabajo de G. Zames [72]). El control $\mathcal{H}^{\infty}$ se volvió un área muy activa, la cual ha sido estudiada desde diferentes puntos de vista, algunos de ellos relacionados con problemas en espacios con métrica indefinida. En particular, la introducción de los espacios de Krein propuesta por B. Hassibi, A. H. Sayed y T. Kailath en técnicas de estimación y control $\mathcal{H}^{\infty}$ permitió adaptar herramientas tradicionales de la teoría de control LQG al control $\mathcal{H}^{\infty}$ (en [42] hay una completa exposición sobre este tema). Además, la introducción de los espacios de Krein otorgó una explicación a algunos aspectos importantes en la teoría de filtrado adaptativo, ver [39] y [41].

Algunos de estos problemas (planteados en espacios de dimensión finita) pueden interpretarse adecuadamente como problemas de cuadrados mínimos indefinidos. Dada una matriz $A \in \mathbb{R}^{m \times n}$ con $m \geq n$ y un vector $y \in \mathbb{R}^{m}$, una solución de cuadrados mínimos indefinidos de la ecuación $A x=y$ es un vector $u \in \mathbb{R}^{n}$ tal que

$$
(A u-y)^{t} J(A u-y)=\operatorname{mí}_{x \in \mathbb{R}^{n}}(A x-y)^{t} J(A x-y),
$$

donde $J=\left(\begin{array}{cc}I_{p} & 0 \\ 0 & -I_{q}\end{array}\right)$ es una matriz de signatura con $p+q=m$. La descripción de las soluciones de cuadrados mínimos indefinidos de $A x=y$ está relacionada con una técnica de estimación lineal en espacios de Krein, estudiada por S. Chandrasekaran et al. [14] y A. H. Sayed et al. [66].

Análogamente, dados dos espacios de Hilbert $\mathcal{H}$ y $\mathcal{K}$, un operador de rango cerrado $C \in$ 
$L(\mathcal{H}, \mathcal{K})$, un operador autoadjunto $B \in L(\mathcal{K})$ y un vector $y \in \mathcal{K}$, diremos que un vector $u \in \mathcal{H}$ es una $B$-solución de cuadrados mínimos (y lo anotaremos $B$-LSS) de la ecuación $C x=y$ si satisface

$$
\langle B(C u-y), C u-y\rangle=\min _{x \in \mathcal{H}}\langle B(C x-y), C x-y\rangle .
$$

En particular, si $B=I$ la ecuación anterior define el problema clásico de cuadrados mínimos, i.e. calcular $u \in \mathcal{H}$ tal que

$$
\|C u-y\|^{2}=\min _{x \in \mathcal{H}}\|C x-y\|^{2} .
$$

Este último problema siempre admite una (única) solución de norma mínima, dada por $u=C^{\dagger} y$, siendo $C^{\dagger}$ la inversa de Moore-Penrose de $C$. Por otra parte, si $B$ es una reflexión, i.e. $B=B^{*}=$ $B^{-1}$, la Ec. (5.0.1) presenta un problema de optimización en espacios de Krein similar a aquellos considerados anteriormente en dimensión finita.

Cuando consideramos la forma sesquilineal $\langle x, y\rangle_{B}=\langle B x, y\rangle$ inducida por un operador autoadjunto arbitario $B \in L(\mathcal{K})$, ni la existencia ni la unicidad de $B$-LSS están garantizadas en general, aún cuando $\mathcal{H}$ tiene dimensión finita. De hecho, la existencia de $B$-LSS de la ecuación $C x=y$ resulta estar intimamente relacionada con la compatibilidad del par conformado por el operador $B$ y el rango del operador $C$.

En [18], utilizando estas ideas y suponiendo que el operador $B$ es (semidefinido) positivo, G. Corach y A. Maestripieri estudiaron el problema de cuadrados mínimos pesados. El presente capítulo puede entenderse como una extensión de dicho trabajo a operadores autoadjuntos.

\subsection{El problema de cuadrados mínimos indefinidos}

Dado un operador $C \in C R(\mathcal{H}, \mathcal{K})$ y un vector $y \in \mathcal{K}$, una solución pesada de cuadrados mínimos de la ecuación $C x=y$ es un vector $u \in \mathcal{H}$ tal que

$$
\|C u-y\|_{A}=\min _{x \in \mathcal{H}}\|C x-y\|_{A},
$$

siendo $A \in L(\mathcal{K})^{+}$y \|\|$_{A}$ la seminorma sobre $\mathcal{K}$ definida por $\|x\|_{A}=\left\|A^{1 / 2} x\right\|=\langle A x, x\rangle^{1 / 2}$ (ver [18]). Notemos que la Ec. (5.1.1) es equivalente a

$$
\langle A(C u-y), C u-y\rangle=\min _{x \in \mathcal{H}}\langle A(C x-y), C x-y\rangle \text {. }
$$

En esta sección, estudiaremos el problema análogo considerando pesos autoadjuntos. Dados $C \in C R(\mathcal{H}, \mathcal{K}), y \in \mathcal{K}$ y un operador $B \in L(\mathcal{K})^{s}$, nos interesa caracterizar, si existe alguno, aquellos vectores $u \in \mathcal{H}$ tales que

$$
\langle B(C u-y), C u-y\rangle=\min _{x \in \mathcal{H}}\langle B(C x-y), C x-y\rangle .
$$

Estableceremos condiciones necesarias y suficientes para asegurar la existencia de vectores $u \in$ $\mathcal{H}$ que satisfagan la ecuación anterior; y, en este caso, parametrizaremos el conjunto de tales soluciones.

Esa clase de problemas ha sido estudiada anteriormente; por ejemplo, por A. H. Sayed et al. [66]. Dadas dos matrices autoadjuntas inversibles $\Pi$ y $W$, un vector columna $y$, y una matriz 
arbitraria $T$ de dimensiones apropiadas, ellos estudiaron el siguiente problema de minimización: caracterizar aquellos vectores $z_{0}$ tales que

$$
z_{0}^{*} \Pi^{-1} z_{0}+\left(y-T z_{0}\right)^{*} W^{-1}\left(y-T z_{0}\right)=\min _{z}\left[z^{*} \Pi^{-1} z+(y-T z)^{*} W^{-1}(y-T z)\right] .
$$

Observemos que, si $\mathcal{H}_{1}$ y $\mathcal{H}_{2}$ son espacios de Hilbert de dimensión finita, $\Pi \in G L\left(\mathcal{H}_{1}\right)^{s}, W \in$ $G L\left(\mathcal{H}_{2}\right)^{s}, y \in \mathcal{H}_{2}$ y $T \in L\left(\mathcal{H}_{1}, \mathcal{H}_{2}\right)$, el problema anterior puede reformularse como: caracterizar aquellos vectores $z_{0} \in \mathcal{H}_{1}$ tales que

$$
\left\langle B\left(w-C z_{0}\right), w-C z_{0}\right\rangle=\min _{z \in \mathcal{H}_{1}}\langle B(w-C z), w-C z\rangle,
$$

siendo

$w=\left(\begin{array}{l}0 \\ y\end{array}\right) \in \mathcal{H}_{1} \oplus \mathcal{H}_{2}, B=\left(\begin{array}{cc}\Pi^{-1} & 0 \\ 0 & W^{-1}\end{array}\right) \in G L\left(\mathcal{H}_{1} \oplus \mathcal{H}_{2}\right)^{s}$ y $C=\left(\begin{array}{c}I \\ T\end{array}\right) \in L\left(\mathcal{H}_{1}, \mathcal{H}_{1} \oplus \mathcal{H}_{2}\right)$.

Definición. Dados $C \in C R(\mathcal{H}, \mathcal{K}), B \in L(\mathcal{K})^{s}$ e $y \in \mathcal{K}$, un elemento $u \in \mathcal{H}$ es una $B$-solución de cuadrados mínimos de la ecuación $C x=y$ (lo abreviaremos $B$-LSS, por las iniciales de " $B$-least square solution") si

$$
\langle C u-y, C u-y\rangle_{B}=\operatorname{mí}_{x \in \mathcal{H}}\langle C x-y, C x-y\rangle_{B} .
$$

En primer lugar, presentaremos condiciones necesarias y suficientes para garantizar la existencia de $B$-LSS de la ecuación $C x=y$. Resultados similares fueron demostrados con anterioridad para operadores positivos [18, Observación 4.3] y para espacios con métrica indefinida $[9$, Teorema 8.4].

Lema 5.1.1. Sean $C \in C R(\mathcal{H}, \mathcal{K}), B \in L(\mathcal{K})^{s}$ e $y \in \mathcal{K}$. Luego, $u \in \mathcal{H}$ es una $B$-LSS de la ecuación $C x=y$ si y sólo si $R(C)$ es $B$-no negativo y además $y-C u \in R(B C)^{\perp}$.

Demostración. Sea $u \in \mathcal{H}$ una $B$-LSS de $C x=y$. Si $x \in \mathcal{H}$ y $\alpha \in \mathbb{R}$, entonces

$$
\begin{aligned}
\langle C u-y, C u-y\rangle_{B} & \leq\langle C u+\alpha C x-y, C u+\alpha C x-y\rangle_{B}= \\
& =\langle C u-y, C u-y\rangle_{B}+2 \alpha \operatorname{Re}\langle C u-y, C x\rangle_{B}+\alpha^{2}\langle C x, C x\rangle_{B} .
\end{aligned}
$$

Luego, $2 \alpha \operatorname{Re}\langle C u-y, C x\rangle_{B}+\alpha^{2}\langle C x, C x\rangle_{B} \geq 0$ para todo $\alpha \in \mathbb{R}$, y un argumento standard prueba que $\operatorname{Re}\langle C u-y, C x\rangle_{B}=0$. De la misma manera, considerando $\beta=i \alpha, \alpha \in \mathbb{R}$, podemos asegurar que $\operatorname{Im}\langle C u-y, C x\rangle_{B}=0$. Por lo tanto, $\langle C u-y, C x\rangle_{B}=0$ y $\langle C x, C x\rangle_{B} \geq 0$ para todo $x \in \mathcal{H}$.

Recíprocamente, supongamos que $R(C)$ es $B$-no negativo y que existe un vector $u \in \mathcal{H}$ tal que $y-C u \in R(B C)^{\perp}$. Luego, para todo $x \in \mathcal{H}$,

$$
\langle y-C x, y-C x\rangle_{B}=\langle y-C u, y-C u\rangle_{B}+\langle C(u-x), C(u-x)\rangle_{B} \geq\langle y-C u, y-C u\rangle_{B} .
$$

Por lo tanto, $u$ es una $B$-LSS de $C x=y$.

Corolario 5.1.2. Sean $C \in C R(\mathcal{H}, \mathcal{K}), B \in L(\mathcal{K})^{s}$ e $y \in \mathcal{K}$. Si $u, v \in \mathcal{H}$ son dos B-LSS de la ecuación $C x=y$, entonces $C(u-v) \in \mathcal{N}=R(C) \cap R(B C)^{\perp}$.

Demostración. Si $u$ y $v$ son $B$-LSS de $C x=y$ entonces, por el Lema 5.1.1, $y-C u, y-C v \in$ $R(B C)^{\perp}$. Por lo tanto,

$$
C(u-v)=(y-C v)-(y-C u) \in R(C) \cap R(B C)^{\perp}=\mathcal{N} .
$$




\subsubsection{La ecuación normal}

Dados $C \in C R(\mathcal{H}, \mathcal{K}), B \in L(\mathcal{K})^{s}$ e $y \in \mathcal{K}$, el Lema 5.1.1 nos motiva a estudiar aquellos $u \in \mathcal{H}$ tales que $y-C u \in R(B C)^{\perp}$. Observemos que, fijado un vector $u \in \mathcal{H}, y-C u \in R(B C)^{\perp}$ si y sólo si $\langle y-C u, C x\rangle_{B}=0$ para todo $x \in \mathcal{H}$, o equivalentemente, $\left\langle C^{*} B(C u-y), x\right\rangle=0$ para todo $x \in \mathcal{H}$. Luego, $y-C u \in R(B C)^{\perp}$ si y sólo si $u$ es una solución de la ecuación normal

$$
C^{*} B(C x-y)=0
$$

Es decir,

Proposición 5.1.3. Sean $C \in C R(\mathcal{H}, \mathcal{K})$ y $B \in L(\mathcal{K})^{s}$. Dado un vector $y \in \mathcal{K}$, el vector $u \in \mathcal{H}$ es solución de la ecuación normal $C^{*} B(C x-y)=0$ si y sólo si $y-C u \in R(B C)^{\perp}$.

La proposición anterior muestra además que, fijado un vector $y \in \mathcal{K}$, existe una solución de la ecuación normal $C^{*} B(C x-y)=0$ si y sólo si

$$
y \in R(C)+R(B C)^{\perp} .
$$

Además, en este caso, el conjunto de soluciones de la ecuación normal puede parametrizarse como una variedad afín paralela al núcleo de un operador acotado.

Proposición 5.1.4. Supongamos que la ecuación normal $C^{*} B(C x-y)=0$ admite una solución $u_{0} \in \mathcal{H}$. Luego, el conjunto de soluciones de $C^{*} B(C x-y)=0$ coincide con

$$
u_{0}+N\left(C^{*} B C\right)
$$

Demostración. La prueba es muy simple ya que, si $v \in N\left(C^{*} B C\right)$ y consideramos el vector $u=u_{0}+v$,

$$
C^{*} B(C u-y)=C^{*} B\left(C u_{0}-y\right)+C^{*} B C v=0 .
$$

Recíprocamente, si $u \neq u_{0}$ es otra solución de la Ec. (5.1.3), entonces $C^{*} B C\left(u-u_{0}\right)=C^{*} B(C u-$ $y)-C^{*} B\left(C u_{0}-y\right)$. Es decir, $u \in u_{0}+N\left(C^{*} B C\right)$.

En algunas aplicaciones, resulta útil suponer que, para cada $y \in \mathcal{K}$, la ecuación normal $C^{*} B(C u-y)=0$ admite al menos una solución. Luego, la Ec. (5.1.4) asegura que esta condición es equivalente a la compatibilidad del par $(B, R(C))$ (ver la Ec. (2.1.1) en los Preliminares). Esto es exactamente lo que enunciaremos a continuación:

Corolario 5.1.5. Sean $C \in C R(\mathcal{H}, \mathcal{K})$ y $B \in L(\mathcal{K})^{s}$. Entonces, existe una solución $u \in \mathcal{H}$ de la ecuación normal $C^{*} B(C x-y)=0$ para todo $y \in \mathcal{K}$ si y sólo si el par $(B, R(C))$ es compatible.

Utilizar esta hipótesis nos permitirá dar otra caracterización de las soluciones de la ecuación normal, ahora a partir de ciertas proyecciones $B$-autoadjuntas.

Proposición 5.1.6. Dados $C \in C R(\mathcal{H}, \mathcal{K})$ y $B \in L(\mathcal{K})^{s}$, supongamos que $(B, R(C))$ es compatible. Si $y \in \mathcal{K} \backslash R(C)$, entonces $u \in \mathcal{H}$ es una solución de $C^{*} B(C x-y)=0$ si y sólo si existe una proyección $Q \in \mathcal{P}(B, R(C))$ tal que $C u=Q y$. 
Demostración. Para $y \in \mathcal{K}$ y $u \in \mathcal{H}$ supongamos que existe $Q \in \mathcal{P}(B, R(C))$ tal que $C u=Q y$. Luego, por el Lema 2.1.1, $y-C u=(I-Q) y \in N(Q) \subseteq R(B C)^{\perp}$ y, aplicando la Proposición 5.1.3, vemos que $u$ es una solución de $C^{*} B(C x-y)=0$.

Recíprocamente, fijado $y \in \mathcal{K} \backslash R(C)$, sea $u$ una solución de $C^{*} B(C x-y)=0$. Por la Proposición 5.1.3, $y=C u+z$ con $z \in R(B C)^{\perp}, z \notin R(C)$. Dado que $z \in R(B C)^{\perp} \backslash R(C)$ y $\mathcal{K}=R(C)+R(B C)^{\perp}$, es fácil ver que existe un subespacio cerrado $\mathcal{S}$ de $R(B C)^{\perp}$ tal que $z \in \mathcal{S}$ y $\mathcal{H}=R(C) \dot{+} \mathcal{S}$. Por lo tanto, $Q=P_{R(C) / / \mathcal{S}} \in \mathcal{P}(B, R(C))$ y

$$
Q y=Q(C u+z)=C u \text {. }
$$

Observación 5.1.7. Con las hipótesis de la Proposición 5.1.6,

(a) se sigue de la demostración de la Proposición 5.1.6 que, dado $y \in \mathcal{K}$, si $C u=Q y$ para alguna $Q \in \mathcal{P}(B, R(C))$, entonces $u$ es una solución de la ecuación normal;

(b) la recíproca de la afirmación anterior no es cierta para $y \in R(C)$. En este caso, $Q y=y$ para toda $Q \in \mathcal{P}(B, R(C))$ y el conjunto de soluciones de $C x=y$ es $C^{\dagger} y+N(C)$ pero el conjunto de soluciones de la ecuación normal $C^{*} B(C x-y)=0$ puede parametrizarse como $C^{\dagger} y+N(B C)$ (ver la Proposición 5.1.14 más adelante).

Corolario 5.1.8. Dados $C \in C R(\mathcal{H}, \mathcal{K})$ y $B \in L(\mathcal{K})^{s}$ tales que $(B, R(C))$ es compatible, supongamos que $\mathcal{N}=\{0\}$. Entonces, fijado $y \in \mathcal{K}, u \in \mathcal{H}$ es una solución de $C^{*} B(C x-y)=0$ si $y$ sólo si $C u=P_{B, \mathcal{S}} y$.

Demostración. Si $(B, R(C))$ es compatible y $\mathcal{N}=\{0\}$ entonces $\mathcal{P}(B, R(C))=\left\{P_{B, R(C)}\right\}$ y $N(B C)=N(C)$. Por lo tanto, el corolario se desprende de la Proposición 5.1.6 y la observación anterior.

Finalmente, presentaremos una última caracterización del conjunto de soluciones de la ecuación normal $C^{*} B(C x-y)=0$; ahora en términos de una solución (acotada) de las ecuaciones $C X C=C$ y $B C X=(C X)^{*} B$.

Proposición 5.1.9. Sean $C \in C R(\mathcal{H}, \mathcal{K})$ y $B \in L(\mathcal{K})^{\text {s }}$ tales que el par $(B, R(C))$ es compatible. Dado y $\in \mathcal{K} \backslash R(C)$, $u \in \mathcal{H}$ es una solución de la ecuación normal $C^{*} B(C x-y)=0$ si y sólo si existe una solución $D \in L(\mathcal{K}, \mathcal{H})$ del sistema

$$
C X C=C, \quad B C X=(C X)^{*} B,
$$

que satisface $D y=u$.

Demostración. Dado $y \in \mathcal{K} \backslash R(C)$, supongamos que $u=D y$, con $D \in L(\mathcal{K}, \mathcal{H})$ cumpliendo las ecuaciones $C D C=C$ y $B C D=(C D)^{*} B$. Luego, es fácil ver que $Q=C D$ es una proyección $B$-autoadjunta. Además, $R(Q) \subseteq R(C)=R(C D C) \subseteq R(Q)$ i.e. $Q \in \mathcal{P}(B, R(C))$. Entonces, $C u=C D y=Q y$ con $Q \in \mathcal{P}(B, R(C))$ y, por la Proposición 5.1.6, $u$ es una solución de la ecuación normal.

Recíprocamente, si $u \in \mathcal{H}$ es una solución de $C^{*} B(C x-y)=0$, existe $Q \in \mathcal{P}(B, R(C))$ tal que $C u=Q y$. Luego, $u=C^{\dagger} Q y+z$ para algún $z \in N(C)$. Consideremos un operador $T \in L(\mathcal{K}, \mathcal{H})$ 
con $R(T) \subseteq N(C)$ tal que $T y=z$, y definamos $D=C^{\dagger} Q+T$. Como $C D=C\left(C^{\dagger} Q+T\right)=Q$ es fácil ver que $D$ es una solución de

$$
C X C=C, \quad B C X=(C X)^{*} B
$$

y cumple $D y=C^{\dagger} Q y+T y=u$.

Como afirmamos en el Lema 5.1.1, una condición necesaria para asegurar la existencia de $B$-LSS de la ecuación $C x=y$ es que $R(C)$ sea $B$-no negativo. El siguiente teorema describe el problema de cuadrados mínimos indefinidos suponiendo esta hipótesis.

Teorema 5.1.10. Dado $B \in L(\mathcal{K})^{s}$, sea $C \in C R(\mathcal{H}, \mathcal{K})$ tal que $R(C)$ es $B$-no negativo. Luego,

1. dado $y \in \mathcal{K}, u \in \mathcal{H}$ es una $B-L S S$ de la ecuación $C x=y$ si y sólo si u es una solución de la ecuación normal $C^{*} B(C x-y)=0$;

2. existe una $B-L S S$ de la ecuación $C x=y$ para todo $y \in \mathcal{K}$ si y sólo si el par $(B, R(C))$ es compatible. En este caso, si $y \in \mathcal{K} \backslash R(C), u \in \mathcal{H}$ es una $B-L S S$ de $C x=y$ si y sólo si existe $Q \in \mathcal{P}(B, R(C))$ tal que $C u=Q y$.

Demostración. Este teorema es la conclusión a la que arribamos al interpretar los resultados anteriores en el caso en que $R(C)$ sea $B$-no negativo.

El siguiente resultado muestra que, si $Q$ es una proyección $B$-autoadjunta con rango $B$-no negativo, ésta se comporta de manera análoga a una proyección ortogonal (con respecto al producto interno del espacio de Hilbert $\mathcal{H}$ ) en el siguiente sentido: fijado un vector $x \in \mathcal{H}, Q x$ resulta un mínimo para un funcional lineal definido sobre $\mathcal{H}$.

Corolario 5.1.11. Sean $Q \in L(\mathcal{H})$ una proyección B-autoadjunta y $x \in \mathcal{H}$. Si $R(Q)$ es $B$-no negativo entonces

$$
\langle(I-Q) x,(I-Q) x\rangle_{B}=\operatorname{mín}_{s \in R(Q)}\langle x-s, x-s\rangle_{B} .
$$

Más aún, si $R(Q)$ es $B$-positivo, $Q x$ es el único vector en $R(Q)$ que alcanza el valor mínimo.

Demostración. Es una consecuencia del teorema anterior, considerando la $B$-LSS de la ecuación $P z=x$, donde $P \in L(\mathcal{H})$ es la proyección ortogonal sobre $R(Q)$.

Observación 5.1.12. Dados $C \in C R(\mathcal{H}, \mathcal{K})$ y $B \in L(\mathcal{K})^{s}$, si $R(C)$ es un subespacio $B$-no positivo de $\mathcal{K}$, los vectores $u \in \mathcal{H}$ que satisfacen

$$
\langle B(C u-y), C u-y\rangle=\operatorname{máx}_{x \in \mathcal{H}}\langle B(C x-y), C x-y\rangle \quad \text { para todo } x \in \mathcal{H},
$$

pueden caracterizarse siguiendo las mismas ideas que utilizamos para resolver el problema de $B$-cuadrados mínimos. De hecho, resultados similares al Teorema 5.1.10 y al Corolario 5.1.11 pueden demostrarse mutatis mutandis. 


\subsubsection{El conjunto de $B$-soluciones de cuadrados mínimos}

Dados los operadores $C \in C R(\mathcal{H}, \mathcal{K})$ y $B \in L(\mathcal{K})^{s}$, supongamos que $(B, R(C))$ es compatible. Luego, si $y \in \mathcal{K}$, es posible caracterizar, cuando existen, todas las soluciones de la ecuación normal $C^{*} B(C x-y)=0$. Con este propósito, definimos una solución particular de la ecuación normal a partir de la proyección distinguida $P_{B, R(C)} \in \mathcal{P}(B, R(C))$.

Definición. Sean $C \in C R(\mathcal{H}, \mathcal{K}), B \in L(\mathcal{K})^{s}$ e $y \in \mathcal{K}$. Supongamos que el par $(B, R(C))$ es compatible. Luego,

$$
u_{y}:=C^{\dagger} P_{B, R(C)} y
$$

es la solución minimal de la ecuación $C^{*} B(C x-y)=0$.

Observando que $C u_{y}=P_{B, R(C)} y$ y aplicando la Observación 5.1.7 vemos que $u_{y}$ es una solución de $C^{*} B(C x-y)=0$. El siguiente resultado caracteriza la solución minimal de la ecuación $C^{*} B(C x-y)=0$.

Proposición 5.1.13. Sean $C \in C R(\mathcal{H}, \mathcal{K})$ y $B \in L(\mathcal{K})^{\text {s }}$ tales que $(B, R(C))$ es compatible. Dado $y \in \mathcal{K}, u_{y} \in \mathcal{H}$ es la única solución de $C^{*} B(C x-y)=0$ en $N(C)^{\perp}$ que satisface

$$
\left\|y-C u_{y}\right\|=\operatorname{mín}\left\{\|y-C u\|: \text { u es una solución de } C^{*} B(C x-y)=0\right\} .
$$

Demostración. Si $y \in R(C)$ es fácil ver que $u_{y}$ satisface la Ec. (5.1.7). Ahora, supongamos que $y \in \mathcal{K} \backslash R(C)$. Si $u_{0}$ es una solución de $C^{*} B(C x-y)=0$ entonces existe una proyección $Q \in \mathcal{P}(B, R(C))$ tal que $C u_{0}=Q y$ y, por la Proposición 2.1.14,

$$
\left\|y-C u_{0}\right\|=\|(I-Q) y\| \geq\left\|\left(I-P_{B, R(C)}\right) y\right\|=\|y-C u\| .
$$

Luego, $u_{0}$ satisface $\left\|y-C u_{0}\right\|=\operatorname{mín}\left\{\|y-C u\|: u\right.$ es una solución de $\left.C^{*} B(C x-y)=0\right\}$ si y sólo si $Q y=P_{B, R(C)} y$, i.e. $C u_{0}=C u_{y}$, o equivalentemente, $u_{0} \in u_{y}+N(C)$.

Dado $y \in \mathcal{K}$, la próxima proposición presenta una parametrización del conjunto de soluciones de la ecuación normal $C^{*} B(C x-y)=0$ en términos de la solución minimal de $C^{*} B(C x-y)=0$. Con este propósito observemos que, si $(B, R(C))$ es compatible, entonces $N\left(C^{*} B C\right)=N(B C)$.

Proposición 5.1.14. Sean $C \in C R(\mathcal{H}, \mathcal{K}), B \in L(\mathcal{K})^{s}$ e $y \in \mathcal{K}$. Si $(B, R(C))$ es compatible entonces el conjunto de soluciones de $C^{*} B(C x-y)=0$ es la variedad afin

$$
u_{y}+N(B C) .
$$

Demostración. Es una consecuencia de la Proposición 5.1.4 y el comentario anterior.

Considerando un operador $C \in C R(\mathcal{H}, \mathcal{K})$ con rango $B$-no negativo y aplicando el Teorema 5.1 .10 , obtenemos el siguiente corolario de la proposición anterior.

Corolario 5.1.15. Sean $C \in C R(\mathcal{H}, \mathcal{K}), B \in L(\mathcal{K})^{s}$ e $y \in \mathcal{K}$. Supongamos que $(B, R(C))$ es compatible y que $R(C)$ es un subespacio $B$-no negativo. Entonces, la variedad afín

$$
u_{y}+N(B C)
$$

coincide con el conjunto de B-LSS de la ecuación $C x=y$. 
Corolario 5.1.16. Sean $C \in C R(\mathcal{H}, \mathcal{K}), B \in L(\mathcal{K})^{s}$ e $y \in \mathcal{K}$. Supongamos que $(B, R(C))$ es compatible y $R(C)$ es $B$-no negativo. Entonces, las siguientes condiciones son equivalentes:

1. $\mathcal{N}=\{0\} ;$

2. $R(C)$ es B-positivo;

3. $u_{y}$ es la única $B$-LSS de $C x=y$ en $N(C)^{\perp}$.

Demostración. Como $C^{*} B C \in L(\mathcal{H})^{+}, C x \in \mathcal{N}$ si y sólo si $\langle C x, C x\rangle_{B}=0$. Por lo tanto, el item 1 es equivalente al item 2. Por otra parte, por el Corolario 5.1.15, el item 1 es equivalente al item 3.

En [40], dados un espacio de Hilbert (de dimensión finita) $\mathcal{H}$, un operador $B \in L(\mathcal{H})^{s}$, un subespacio (cerrado) $B$-no negativo $\mathcal{S}$ de $\mathcal{H}$ y un vector $y \in \mathcal{H}$, B. Hassibi et al. estudiaron el problema de encontrar vectores $u \in \mathcal{S}$ tales que

$$
\langle u-y, u-y\rangle_{B}=\min _{s \in \mathcal{S}}\langle s-y, s-y\rangle_{B}=\min _{x \in \mathcal{H}}\left\langle P_{\mathcal{S}} x-y, P_{\mathcal{S}} x-y\right\rangle_{B},
$$

siendo $P_{\mathcal{S}}$ la proyección ortogonal sobre $\mathcal{S}$. Ellos estaban particularmente interesados en aquellos casos en los que hay una única solución del problema. Por el Lema 5.1.1, $u \in \mathcal{S}$ satisface la Ec. (5.1.8) si y sólo si $y-u \in R\left(B P_{\mathcal{S}}\right)^{\perp}$. Es fácil ver que esta condición se satisface si y sólo si

$$
P_{\mathcal{S}} B P_{\mathcal{S}} u=P_{\mathcal{S}} B y \text {. }
$$

Cuando $\mathcal{H}$ es un espacio de Hilbert de dimensión finita, si existe una única solución $u \in \mathcal{S}$ de (5.1.9) para algún $y_{0} \in \mathcal{H}$, entonces el operador $\left.P_{\mathcal{S}} B P_{\mathcal{S}}\right|_{\mathcal{S}}$ es inyectivo. Por lo tanto, $\left.P_{\mathcal{S}} B P_{\mathcal{S}}\right|_{\mathcal{S}}$ es inversible, y existe una única solución de (5.1.9) para todo $y \in \mathcal{H}$.

Si $\mathcal{H}$ es un espacio de Hilbert de dimensión infinita esto puede ser falso, ya que $\left.P_{\mathcal{S}} B P_{\mathcal{S}}\right|_{\mathcal{S}}$ puede ser inyectivo sin ser inversible. De hecho, en este caso, existe una solución de la Ec. (5.1.9) para todo $y \in \mathcal{H}$ si y sólo si la ecuación

$$
\left(P_{\mathcal{S}} B P_{\mathcal{S}}\right) X=P_{\mathcal{S}} B
$$

admite una solución en $L(\mathcal{H})$, o equivalentemente, el par $(B, \mathcal{S})$ es compatible, ver la Proposición 2.1.7.

\subsubsection{Minimizando en el conjunto de $B$-LSS de una ecuación lineal}

Recordemos que, dado $y \in \mathcal{K}$, el problema de cuadrados mínimos clásico (en espacios de Hilbert) asociado a la ecuación $C x=y$ admite una (única) solución de norma mínima, más precisamente, aquella definida por $u=C^{\dagger} y$.

En los siguientes párrafos estudiaremos un problema de minimización en el conjunto de $B$ LSS de $C x=y$. Dados $y \in \mathcal{K}, C \in C R(\mathcal{H}, \mathcal{K}), B_{1} \in L(\mathcal{H})^{s}, B_{2} \in L(\mathcal{K})^{s}$ tales que $\left(B_{2}, R(C)\right)$ es compatible y $R(C)$ es $B_{2}$-no negativo, consideremos el conjunto $u_{y}+N\left(B_{2} C\right)$ de $B_{2}$-LSS de la ecuación $C x=y$. Intentaremos caracterizar aquellas $B_{2}$-LSS $w \in \mathcal{H}$ de $C x=y$ tales que

$$
\langle w, w\rangle_{B_{1}} \leq\langle u, u\rangle_{B_{1}}, \quad \text { para todo } u \in u_{y}+N\left(B_{2} C\right) .
$$

En lo que sigue, denotaremos $\mathcal{N}_{2}=R(C) \cap R\left(B_{2} C\right)^{\perp}$. Observemos que, si $\left(B_{2}, R(C)\right)$ es compatible entonces $\mathcal{N}_{2}=N\left(B_{2} C\right)$. 
Definición. Un elemento $w \in \mathcal{H}$ es una $B_{1} B_{2}$-solución de cuadrados mínimos (ó brevemente, una $B_{1} B_{2}$-LSS) de $C x=y$ si $w$ es una $B_{2}$-LSS de $C x=y$ y

$$
\langle w, w\rangle_{B_{1}} \leq\langle u, u\rangle_{B_{1}}
$$

para toda $B_{2}$-LSS $u$ de $C x=y$.

Proposición 5.1.17. Sean $C \in C R(\mathcal{H}, \mathcal{K}), B_{1} \in L(\mathcal{H})^{s}$ y $B_{2} \in L(\mathcal{K})^{s}$ tales que $\left(B_{2}, R(C)\right)$ es compatible y $R(C)$ es $B_{2}$-no negativo. Luego, existe una $B_{1} B_{2}-L S S$ de la ecuación $C x=y$ para todo y $\in \mathcal{K}$ si y sólo si $\left(B_{1}, N\left(B_{2} C\right)\right)$ es compatible y $N\left(B_{2} C\right)$ es $B_{1}$-no negativo. Además, si $y \in \mathcal{K} \backslash R\left(B_{2} C\right)^{\perp}$, w es una $B_{1} B_{2}-L S S$ de $C x=y$ si y sólo si existen una $Q \in \mathcal{P}\left(B_{1}, N\left(B_{2} C\right)\right)$ y una $P \in \mathcal{P}\left(B_{2}, R(C)\right)$ tales que

$$
w=(I-Q) C^{\dagger} P y
$$

Demostración. En primer lugar, supongamos que, para todo $y \in \mathcal{K}$, existe una $B_{1} B_{2}$-LSS $w_{y} \in \mathcal{H}$ de la ecuación $C x=y$, i.e.

$$
\left\langle w_{y}, w_{y}\right\rangle_{B_{1}}=\operatorname{mín}_{u \in u_{y}+N\left(B_{2} C\right)}\langle u, u\rangle_{B_{1}}
$$

donde $u_{y}=C^{\dagger} P_{B_{2}, R(C)} y$ es la $B_{2}$-LSS minimal de la ecuación $C x=y$. Para cada $y \in \mathcal{K}$, sea $z_{y} \in N\left(B_{2} C\right)$ tal que $w_{y}=u_{y}+z_{y}=u_{y}+E z_{y}$, siendo $E=P_{N\left(B_{2} C\right)}$ la proyección ortogonal sobre $N\left(B_{2} C\right)$. Luego,

$$
\begin{aligned}
\left\langle u_{y}+E z_{y}, u_{y}+E z_{y}\right\rangle_{B_{1}} & =\left\langle w_{y}, w_{y}\right\rangle_{B_{1}}=\min _{z \in N\left(B_{2} C\right)}\left\langle u_{y}+z, u_{y}+z\right\rangle_{B_{1}}= \\
& =\operatorname{mín}_{x \in \mathcal{H}}\left\langle u_{y}+E x, u_{y}+E x\right\rangle_{B_{1}} .
\end{aligned}
$$

Por lo tanto, $z_{y}$ es una $B_{1}$-LSS de la ecuación $E x=-u_{y}$. Entonces, por el Lema 5.1.1, $R(E)=$ $N\left(B_{2} C\right)$ es $B_{1}$-no negativo.

La compatibilidad del par $\left(B_{2}, R(C)\right)$ asegura que $\left\{u_{y}: y \in \mathcal{K}\right\}=N(C)^{\perp}$, es decir, la ecuación $E x=z$ admite una $B_{1}$-LSS para todo $z \in N(C)^{\perp}$. Además, la ecuación $E x=z$ admite una solución exacta - la cual resulta ser también una $B_{1}$-LSS - para todo $z \in N(C)$, porque $N(C) \subseteq R(E)$ es un subespacio $B_{1}$-no negativo. Por lo tanto, $E x=z$ admite una $B_{1}$-LSS para todo $z \in \mathcal{K}$ y, aplicando el Teorem 5.1.10, tenemos que el par $\left(B_{1}, N\left(B_{2} C\right)\right)$ es compatible.

Observemos que, si $y \notin R\left(B_{2} C\right)^{\perp}$ entonces $u_{y} \notin R(E)=N\left(B_{2} C\right)$ : De hecho, $u_{y} \in N\left(B_{2} C\right)$ si y sólo si $B_{2} P_{B_{2}, R(C)} y=0$ y $N\left(B_{2} P_{B_{2}, R(C)}\right)=R\left(B_{2} P_{B_{2}, R(C)}\right)^{\perp}=R\left(B_{2} C\right)^{\perp}$. Luego, dado $y \in \mathcal{K} \backslash R\left(B_{2} C\right)^{\perp}$, por el Teorema 5.1.10, existe una $Q \in \mathcal{P}\left(B_{1}, N\left(B_{2} C\right)\right)$ tal que $E z_{y}=-Q u_{y}$. Por lo tanto,

$$
w_{y}=u_{y}+z_{y}=u_{y}+E z_{y}=(I-Q) u_{y}=(I-Q) C^{\dagger} P_{B_{2}, R(C)} y .
$$

Recíprocamente, supongamos que $\left(B_{1}, N\left(B_{2} C\right)\right)$ es compatible, $N\left(B_{2} C\right)$ es $B_{1}$-no negativo y sea $Q \in \mathcal{P}\left(B_{1}, N\left(B_{2} C\right)\right)$. Si $P \in \mathcal{P}\left(B_{2}, R(C)\right)$, notemos que $(I-Q) C^{\dagger} P=(I-Q) C^{\dagger} P_{B_{2}, R(C)}$. De hecho, si $P \in \mathcal{P}\left(B_{2}, R(C)\right)$, existe un operador $Z \in L\left(R(C)^{\perp}, \mathcal{N}_{2}\right)$ tal que $P=P_{B_{2}, R(C)}+Z$ (ver la Proposición 2.1.12). Entonces, $(I-Q) C^{\dagger} P=(I-Q) C^{\dagger} P_{B_{2}, R(C)}$ porque $(I-Q) C^{\dagger} Z=0$.

Dado $y \in \mathcal{K}$, consideremos el vector $w=(I-Q) C^{\dagger} P_{B_{2}, R(C)} y$. Luego, $w \in u_{y}+N\left(B_{2} C\right)$ y, por el Corolario 5.1.15, es una $B_{2}$-LSS de $C x=y$. 
Por otra parte, dada cualquier $B_{2}$-LSS $u$ de $C x=y$, existe un vector $z \in N\left(B_{2} C\right)$ tal que $u=u_{y}+z=u_{y}+Q z \mathrm{y}$

$$
\begin{aligned}
\langle u, u\rangle_{B_{1}} & =\left\langle(I-Q) u_{y}+Q\left(u_{y}+z\right),(I-Q) u_{y}+Q\left(u_{y}+z\right)\right\rangle_{B_{1}}= \\
& =\langle w, w\rangle_{B_{1}}+2 \operatorname{Re}\left\langle(I-Q) u_{y}, Q\left(u_{y}+z\right)\right\rangle_{B_{1}}+\left\langle Q\left(u_{y}+z\right), Q\left(u_{y}+z\right)\right\rangle_{B_{1}}= \\
& =\langle w, w\rangle_{B_{1}}+\left\langle Q\left(u_{y}+z\right), Q\left(u_{y}+z\right)\right\rangle_{B_{1}} \geq\langle w, w\rangle_{B_{1}},
\end{aligned}
$$

porque $R(Q) \perp_{B_{1}} N(Q)$ y $R(Q)=N\left(B_{2} C\right)$ es $B_{1}$-no negativo. Por lo tanto, $w$ es una $B_{1} B_{2}$-LSS de $C x=y$.

Como consecuencia de la demostración de la Proposición 5.1.17 tenemos que, dado y $\in$ $\mathcal{K} \backslash R\left(B_{2} C\right)^{\perp}$, el conjunto de $B_{1} B_{2}$-LSS de la ecuación $C x=y$ es

$$
\left\{(I-Q) C^{\dagger} P_{B_{2}, R(C)} y: Q \in \mathcal{P}\left(B_{1}, N\left(B_{2} C\right)\right)\right\} .
$$

Por otra parte, si $y \in R\left(B_{2} C\right)^{\perp}$ entonces $u_{y} \in N\left(B_{2} C\right)$ ya que

$$
B_{2} C u_{y}=B_{2} C P_{B_{2}, R(C)} y=\left(P_{B_{2}, R(C)}\right)^{*} B_{2} y,
$$

y $N\left(\left(P_{B_{2}, R(C)}\right)^{*}\right)=R\left(P_{B_{2}, R(C)}\right)^{\perp}=R(C)^{\perp}$. Por lo que, dado $y \in R\left(B_{2} C\right)^{\perp}$, al problema de caracterizar las $B_{1} B_{2}$-LSS de $C x=y$ podemos traducirlo en encontrar aquellos $u \in N\left(B_{2} C\right)$ tales que $\langle u, u\rangle_{B_{1}}=0$ (ver la Ec. (5.1.10)), es decir, el conjunto de $B_{1} B_{2}$-LSS es

$$
\mathcal{N}_{1}=N\left(B_{1}\right) \cap N\left(B_{2} C\right) .
$$

Corolario 5.1.18. Sean $C \in C R(\mathcal{H}, \mathcal{K}), B_{1} \in L(\mathcal{H})^{s}$ y $B_{2} \in L(\mathcal{K})^{s}$ tales que $\left(B_{2}, R(C)\right)$ es compatible y $R(C)$ es $B_{2}$-no negativo. Supongamos que $\left(B_{1}, N\left(B_{2} C\right)\right)$ es compatible, $N\left(B_{2} C\right)$ es $B_{1}$-no negativo y $\mathcal{N}_{1}=\{0\}$. Si $y \in \mathcal{K}$ entonces, $w$ es una $B_{1} B_{2}-L S S$ de $C x=y$ si y sólo si

$$
w=\left(I-P_{B_{1}, N\left(B_{2} C\right)}\right) C^{\dagger} P_{B_{2}, R(C)} y .
$$

Como mencionamos anteriormente, si $y \in R\left(B_{2} C\right)^{\perp}$, la Ec. (5.1.11) describe un subconjunto propio del conjunto de todas las $B_{1} B_{2}$-LSS de $C x=y$. Sin embargo, este subconjunto contiene a la $B_{1} B_{2}$-LSS de norma mínima.

Proposición 5.1.19. Sean $C \in C R(\mathcal{H}, \mathcal{K}), B_{1} \in L(\mathcal{H})^{s}$ y $B_{2} \in L(\mathcal{K})^{s}$ tales que $\left(B_{2}, R(C)\right)$ es compatible y $R(C)$ es un subespacio $B_{2}$-no negativo de $\mathcal{K}$. Supongamos además que el par $\left(B_{1}, N\left(B_{2} C\right)\right)$ es compatible y $N\left(B_{2} C\right)$ es un subespacio $B_{1}$-no negativo de $\mathcal{H}$. Si $y \in \mathcal{K}$ entonces $v_{y}=\left(I-P_{B_{1}, N\left(B_{2} C\right)}\right) C^{\dagger} P_{B_{2}, R(C)}$ y es el único elemento de norma mínima en el conjunto de $B_{1} B_{2}-L S S$ de $C x=y$.

Demostración. Si $y \in \mathcal{K} \backslash R\left(B_{2} C\right)^{\perp}$ y $v$ es una $B_{1} B_{2}$-LSS de $C x=y$, sabemos que existe $Q \in \mathcal{P}\left(B_{1}, N\left(B_{2} C\right)\right)$ tal que $v=(I-Q) u_{y}$ y, por la Proposición 2.1.14,

$$
\|v\|=\left\|(I-Q) u_{y}\right\| \geq\left\|\left(I-P_{B_{1}, N\left(B_{2} C\right)}\right) u_{y}\right\|=\left\|v_{y}\right\| .
$$

Por lo tanto, $v_{y}=\left(I-P_{B_{1}, N\left(B_{2} C\right)}\right) C^{\dagger} P_{B_{2}, R(C)} y$ es el único elemento de norma mínima en el conjunto de $B_{1} B_{2}$-LSS de $C x=y$.

Por otra parte, si $y \in R\left(B_{2} C\right)^{\perp}$ tenemos que $u_{y} \in N\left(B_{2} C\right)$ y, en consecuencia, el vector $v_{y}=\left(I-P_{B_{1}, N\left(B_{2} C\right)}\right) C^{\dagger} P_{B_{2}, R(C)} y$ tiene norma mínima porque $v_{y}=0$. 


\subsection{Inversas generalizadas pesadas}

Dados $C \in C R(\mathcal{H}, \mathcal{K})$ y $B_{2} \in L(\mathcal{K})^{s}$, supongamos que $\left(B_{2}, R(C)\right)$ es compatible y $R(C)$ es $B_{2}$-positivo. Luego, por el Corolario 5.1.16, tenemos que $\mathcal{N}_{2}=\{0\}$ y en consecuencia $N\left(B_{2} C\right)=$ $N(C)$. Consideremos un operador $B_{1} \in L(\mathcal{H})^{s}$ tal que $\left(B_{1}, N(C)\right)$ es compatible y $N(C)$ es $B_{1}$-no negativo. Entonces, por la Proposición 5.1.17, $w \in \mathcal{H}$ es una $B_{1} B_{2}$-LSS de la ecuación $C x=y$ si y sólo si

$$
w=(I-Q) C^{\dagger} P_{B_{2}, R(C)} y,
$$

siendo $Q \in \mathcal{P}\left(B_{1}, N(C)\right)$. Esto nos motiva a estudiar el siguiente conjunto de operadores:

$$
\left\{(I-Q) C^{\dagger} P: Q \in \mathcal{P}\left(B_{1}, N(C)\right) \text { and } P \in \mathcal{P}\left(B_{2}, R(C)\right)\right\} .
$$

Sean $Q \in \mathcal{P}\left(B_{1}, N(C)\right), P \in \mathcal{P}\left(B_{2}, R(C)\right)$ y consideremos $D=(I-Q) C^{\dagger} P$. Luego, a partir de las identidades $C^{\dagger} C C^{\dagger} P=P$ y $(I-Q) C^{\dagger} C=(I-Q)$, es fácil ver que

$$
C D=P \text { and } D C=I-Q .
$$

Por lo tanto, $D$ es una inversa generalizada pesada de $C$ en el siguiente sentido:

Definición. Dado un operador $C \in C R(\mathcal{H}, \mathcal{K})$ y pesos $B_{1} \in L(\mathcal{H})^{s}$ y $B_{2} \in L(\mathcal{K})^{s}, D \in L(\mathcal{K}, \mathcal{H})$ es una inversa generalizada pesada de $C$ si $D$ es una solución de

$$
C X C=C, \quad X C X=X, \quad B_{1}(X C)=(X C)^{*} B_{1}, \quad B_{2}(C X)=(C X)^{*} B_{2} .
$$

Las ecuaciones anteriores pueden entenderse como una extensión de las definiciones previas, dadas para pesos positivos, por L. Eldén [32] (en espacios de dimensión finita) y por G. Corach y A. Maestripieri [18] (para espacios de Hilbert de dimensión infinita).

Esta sección está dedicada a presentar condiciones necesarias y suficientes para la existencia de inversas generalizadas pesadas (respecto a pesos autoadjuntos $B_{1}$ y $B_{2}$ ) de un operador $C$ con rango cerrado, y a caracterizar estas inversas en términos de la inversa de Moore-Penrose de $C$ y el conjunto de proyecciones $B_{i}$-autoadjuntas $(i=1,2)$. La prueba del siguiente Teorema 5.2.1 es análoga a la presentada en el caso de pesos positivos en [18, Sección 3].

Teorema 5.2.1. Dados $C \in C R(\mathcal{H}, \mathcal{K}), B_{1} \in L(\mathcal{H})^{s}$ y $B_{2} \in L(\mathcal{K})^{s}$ existe $D \in L(\mathcal{K}, \mathcal{H})$ tal que $D$ es una solución de (5.2.1) si y sólo si $\left(B_{1}, N(C)\right)$ y $\left(B_{2}, R(C)\right)$ son pares compatibles. En este caso,

$$
G I\left(C, B_{1}, B_{2}\right)=\left\{(I-Q) C^{\dagger} P: Q \in \mathcal{P}\left(B_{1}, N(C)\right) \text { and } P \in \mathcal{P}\left(B_{2}, R(C)\right)\right\}
$$

coincide con el conjunto de soluciones de (5.2.1).

Demostración. Supongamos que $\left(B_{1}, N(C)\right)$ y $\left(B_{2}, R(C)\right)$ son pares compatibles. Luego, dadas $P \in \mathcal{P}\left(B_{2}, R(C)\right)$ y $Q \in \mathcal{P}\left(B_{1}, N(C)\right)$, consideremos el operador $D=(I-Q) C^{\dagger} P$ y notemos que, por el comentario que precede a la definición de inversa generalizada pesada,

$$
C D=P \quad \text { y } \quad D C=I-Q .
$$

Por lo tanto, $D$ es una solución de (5.2.1), es decir, es una inversa generalizada pesada de $C$.

Recíprocamente, supongamos que existe una inversa generalizada pesada de $C$. Notemos entonces que $P=C D$ (resp. $E=D C$ ) es una proyección $B_{2}$-autoadjunta (resp. $B_{1}$-autoadjunta). 
Además, $R(P) \subseteq R(C)=R(C D C) \subseteq R(C D)=R(P)$ y $N(C) \subseteq N(E)=N(D C) \subseteq$ $N(C D C)=N(C)$. Por lo tanto, $P$ es una proyección $B_{2}$ autoadjunta con $R(P)=R(C)$ y $I-E$ es una proyección $B_{1}$ autoadjunta con $R(I-E)=N(Q)=N(C)$, es decir, los pares $\left(B_{1}, N(C)\right)$ y $\left(B_{2}, R(C)\right)$ son compatibles.

Además, $D=D C D=D\left(C C^{\dagger} C\right) D=(D C) C^{\dagger}(C D)=E C^{\dagger} P$ y, si llamamos $Q=I-E$, entonces $D=(I-Q) C^{\dagger} P$ con $P \in \mathcal{P}\left(B_{2}, R(C)\right)$ y $Q \in \mathcal{P}\left(B_{1}, N(C)\right)$. Por lo tanto, el conjunto de soluciones acotadas de (5.2.1) coincide con $G I\left(C, B_{1}, B_{2}\right)$.

Para poder caracterizar el conjunto de $B_{1} B_{2}$-LSS de $C x=y$ necesitaremos los siguientes lemas técnicos.

Lema 5.2.2. Sea $C \in C R(\mathcal{H}, \mathcal{K})$ y consideremos un subespacio cerrado $\mathcal{S} \subseteq R(C)$. Entonces, $P_{\mathcal{S}} C \in C R(\mathcal{H}, \mathcal{K}) y$

$$
\left(P_{\mathcal{S}} C\right)^{\dagger}=P_{N\left(P_{\mathcal{S}} C\right)^{\perp}} C^{\dagger} .
$$

Demostración. Dado el operador $\tilde{C}=P_{\mathcal{S}} C \in L(\mathcal{H}, \mathcal{K})$, notemos que $R(\tilde{C})=\mathcal{S}$. Consideremos el operador $T=P_{N(\tilde{C}) \perp} C^{\dagger} \in L(\mathcal{K}, \mathcal{H})$. Luego,

$$
\tilde{C} T=\tilde{C} P_{N(\tilde{C})^{\perp}} C^{\dagger}=\tilde{C} C^{\dagger}=P_{\mathcal{S}} C C^{\dagger}=P_{\mathcal{S}} P_{R(C)}=P_{\mathcal{S}}=P_{R(\tilde{C})} .
$$

Por lo tanto, $\tilde{C} T \tilde{C}=\tilde{C}$. Además, $(T \tilde{C})^{2}=T(\tilde{C} T \tilde{C})=T \tilde{C}$, i.e. $T \tilde{C}$ es una proyección. Como $N(\tilde{C}) \subseteq N(T \tilde{C}) \subseteq N(\tilde{C} T \tilde{C})=N(\tilde{C})$ y $R(T \tilde{C}) \subseteq R(T) \subseteq N(\tilde{C})^{\perp}$, tenemos que $T \tilde{C}=P_{N(\tilde{C})^{\perp}}$. Entonces, $T \tilde{C} T=T$. Por lo tanto, $\left(P_{\mathcal{S}} C\right)^{\dagger}=T=P_{N\left(P_{\mathcal{S}} C\right)^{\perp}} C^{\dagger}$.

Lema 5.2.3. Sean $C \in C R(\mathcal{H}, \mathcal{K}), B_{1} \in L(\mathcal{H})^{s}$ y $B_{2} \in L(\mathcal{K})^{s}$. Si $Q \in \mathcal{P}\left(B_{1}, N\left(B_{2} C\right)\right)$, entonces

$$
(I-Q) C^{\dagger}=(I-Q) \tilde{C}^{\dagger},
$$

siendo $\tilde{C}=P_{R(C) \ominus \mathcal{N}_{2}} C \in L(\mathcal{H}, \mathcal{K})$.

Demostración. Si $\tilde{C}=P_{R(C) \ominus \mathcal{N}_{2}} C$ entonces, por el Lema 5.2.2, $\tilde{C}^{\dagger}=P_{N\left(P_{R(C) \ominus \mathcal{N}_{2}} C\right)^{\perp} C^{\dagger}}=$ $P_{N\left(B_{2} C\right)^{\perp}} C^{\dagger}$ ya que

$$
N\left(P_{R(C) \ominus \mathcal{N}_{2}} C\right)=C^{-1}\left(\mathcal{N}_{2}\right)=N\left(B_{2} C\right) .
$$

Además, si $Q \in \mathcal{P}\left(B_{1}, N\left(B_{2} C\right)\right)$ entonces $(I-Q) P_{N\left(B_{2} C\right)}=0$. Por lo tanto, $(I-Q) \tilde{C}^{\dagger}=$ $(I-Q) P_{N\left(B_{2} C\right)^{\perp}} C^{\dagger}=(I-Q) C^{\dagger}$.

Proposición 5.2.4. Sean $C \in C R(\mathcal{H}, \mathcal{K}), B_{1} \in L(\mathcal{H})^{s}$ y $B_{2} \in L(\mathcal{K})^{s}$ tales que $\left(B_{2}, R(C)\right)$ y $\left(B_{1}, N\left(B_{2} C\right)\right)$ son compatibles, $R(C)$ es $B_{2}$-no negativo y $N\left(B_{2} C\right)$ es $B_{1}$-no negativo. Dado $y \in \mathcal{K} \backslash R\left(B_{2} C\right)^{\perp}$, el conjunto de $B_{1} B_{2}-L S S$ de la ecuación $C x=y$ está dado por

$$
\left\{T y: T \in G I\left(P_{R(C) \ominus \mathcal{N}_{2}} C, B_{1}, B_{2}\right)\right\} .
$$

Demostración. Por la Proposición 5.1.17 sabemos que, dado $y \in \mathcal{K} \backslash R\left(B_{2} C\right)^{\perp}$, el conjunto de $B_{1} B_{2}$-LSS de $C x=y$ es

$$
\left\{(I-Q) C^{\dagger} P y: Q \in \mathcal{P}\left(B_{1}, N\left(B_{2} C\right)\right), P \in \mathcal{P}\left(B_{2}, R(C)\right)\right\} .
$$


Dada $P \in \mathcal{P}\left(B_{2}, R(C)\right)$, sea $E=P_{\mathcal{N}_{2} / / R(C) \ominus \mathcal{N}_{2}+N(P)}$. Si $\tilde{C}=P_{R(C) \ominus \mathcal{N}_{2}} C$ entonces $R(\tilde{C})=$ $R(C) \ominus \mathcal{N}_{2}$ y, de acuerdo a la Proposición 2.1.16, $P=P_{B_{2}, R(\tilde{C})}+E$. Por otra parte, dada cualquier $Q \in \mathcal{P}\left(B_{1}, N\left(B_{2} C\right)\right)$, puede comprobarse que $(I-Q) C^{\dagger} E=0$ ya que $R\left(C^{\dagger} E\right) \subseteq N\left(B_{2} C\right)$. Luego, el conjunto de $B_{1} B_{2}$-LSS de $C x=y$ puede describirse como

$$
\left\{(I-Q) C^{\dagger} P_{B_{2}, R(\tilde{C})} y: Q \in \mathcal{P}\left(B_{1}, N\left(B_{2} C\right)\right)\right\} .
$$

Aplycando el Lema 5.2.3, y gracias a que $\mathcal{P}\left(B_{2}, R(\tilde{C})\right)=\left\{P_{B_{2}, R(\tilde{C})}\right\}$, es fácil ver que este conjunto coincide con $\left\{T y: T \in G I\left(\tilde{C}, B_{1}, B_{2}\right)\right\}$.

\subsection{Una solución minimax para la ecuación $C x=y$}

En la Sección 5.1 establecimos la relación existente entre las $B$-LSS de $C x=y$ y las soluciones de la ecuación normal $C^{*} B(C x-y)=0$. Más precisamente, suponiendo que $R(C)$ es un subespacio $B$-no negativo de $\mathcal{K}$, probamos que $u \in \mathcal{H}$ es una $B$-LSS de $C x=y$ si y sólo si éste es una solución de la ecuación normal $C^{*} B(C x-y)=0$. También mostramos que la ecuación normal $C^{*} B(C x-y)=0$ admite solución para todo $y \in \mathcal{K}$ si y sólo si el par $(B, R(C))$ es compatible, y que las soluciones de la ecuación normal están relacionadas con los elementos de $\mathcal{P}(B, R(C))$.

El propósito de esta sección es estudiar las soluciones de la ecuación normal en el caso en que $R(C)$ no es un subespacio $B$-definido. Recordemos que, por el Lema 5.1.1, en este caso no existen $B$-LSS de la ecuación $C x=y$. Sin embargo, las soluciones de la ecuación normal $C^{*} B(C x-y)=0$ pueden relacionarse con las soluciones de un problema minimax:

Dados $C \in C R(\mathcal{H}, \mathcal{K}), B \in L(\mathcal{K})^{s}$ y un vector $y \in \mathcal{K} \backslash R(C)$, supongamos que $(B, R(C))$ es compatible y $u \in \mathcal{H}$ es una solución de $C^{*} B(C x-y)=0$. Luego, por la Proposición 5.1.6, existe $Q \in \mathcal{P}(B, R(C))$ tal que $C u=Q y$ y, por la Proposición 3.2.14,

$$
\langle y-C u, y-C u\rangle_{B}=\langle(I-Q) y,(I-Q) y\rangle_{B}=\min _{s \in \mathcal{S}_{+}} \operatorname{máx}_{t \in \mathcal{S}_{-}}\langle y-(s+t), y-(s+t)\rangle_{B},
$$

donde $R(C) \ominus \mathcal{N}=R(C) \ominus N(B)=\mathcal{S}_{+} \oplus \mathcal{S}_{-}$es la descomposición dada en el Teorema 2.3.2.

Observemos que esta representación depende de la existencia de una descomposición $B$ ortogonal de $R(C)$. En consecuencia, introduciremos un nuevo concepto relacionado con la métrica indefinida $\langle,\rangle_{B}$ definida sobre $\mathcal{K}$.

Definición 5.3.1. Dado $B \in L(\mathcal{K})^{s}$, diremos que un subespacio cerrado $\mathcal{S}$ de $\mathcal{K}$ es $B$-descomponible si puede ser representado como la suma directa $B$-ortogonal de un subespacio $B$-neutro $\mathcal{S}_{0}$, un subespacio $B$-positivo $\mathcal{S}_{+}$y un subespacio $B$-negativo $\mathcal{S}_{-}$, i.e.

$$
\mathcal{S}=\mathcal{S}_{0} \dot{+} \mathcal{S}_{+} \dot{+} \mathcal{S}_{-}
$$

Cabe destacar que ésta es un caso particular de la definición dada en la sección 1.2.1, por lo tanto, recomendamos al lector referirse a dicha sección para una exposición completa sobre este tema. En principio, notemos que no todo subespacio cerrado $\mathcal{S}$ de $\mathcal{H}$ es $B$-descomponible (ver el Ejemplo 1.2.11) y recordemos además que, si $\mathcal{S}$ es $B$-descomponible, entonces $\mathcal{S}_{0}=\mathcal{N}$. 
Definición. Sean $C \in C R(\mathcal{H}, \mathcal{K})$ y $B \in L(\mathcal{K})^{s}$ tales que $R(C)$ es $B$-descomponible. Dado $y \in \mathcal{K}$, un vector $u \in \mathcal{H}$ es una solución $B$-minimax $(B$-MMS) de la ecuación $C x=y$ si

$$
\begin{aligned}
\langle y-C u, y-C u\rangle_{B} & =\operatorname{mím}_{s \in \mathcal{S}_{+}} \operatorname{máx}_{t \in \mathcal{S}_{-}}\langle y-(s+t), y-(s+t)\rangle_{B}= \\
& =\operatorname{máx}_{t \in \mathcal{S}_{-}} \min _{s \in \mathcal{S}_{+}}\langle y-(s+t), y-(s+t)\rangle_{B}
\end{aligned}
$$

siendo $R(C)=\mathcal{N} \dot{+} \mathcal{S}_{+} \dot{+} \mathcal{S}_{-}$una descomposición como la de la Definición 5.3.1.

Observación 5.3.2. Dada una descomposición $R(C)=\mathcal{N} \dot{+} \mathcal{S}_{+} \dot{+} \mathcal{S}_{-}$como la mencionada anteriormente, notemos que

$$
\operatorname{máx}_{t \in \mathcal{S}_{-}} \min _{s \in \mathcal{S}_{+}}\langle y-(s+t), y-(s+t)\rangle_{B}=\operatorname{máx}_{t \in \mathcal{S}_{-}} \operatorname{mín}_{x \in \mathcal{S}_{+}+\mathcal{N}}\langle y-(x+t), y-(x+t)\rangle_{B} .
$$

De hecho, para un vector $t \in \mathcal{S}_{-}$fijo,

$$
\min _{x \in \mathcal{S}_{+}+\mathcal{N}}\langle y-(x+t), y-(x+t)\rangle_{B} \leq \min _{s \in \mathcal{S}_{+}}\langle y-(s+t), y-(s+t)\rangle_{B}
$$

porque $\mathcal{S}_{+} \subseteq \mathcal{S}_{+}+\mathcal{N}$. Por otra parte, si $w \in \mathcal{S}_{+}+\mathcal{N}$ satisface

$$
\langle y-(w+t), y-(w+t)\rangle_{B}=\min _{x \in \mathcal{S}_{+}+\mathcal{N}}\langle y-(x+t), y-(x+t)\rangle_{B},
$$

por el Lema 5.1.1 tenemos que $y-(w+t)$ es $B$-ortogonal a $\mathcal{S}_{+}+\mathcal{N}$, es decir, $\langle y-(w+t), x\rangle_{B}=$ 0 para todo $x \in \mathcal{S}_{+}+\mathcal{N}$. Supongamos que $w=s_{0}+n_{0}$, con $s_{0} \in \mathcal{S}_{+}$y $n_{0} \in \mathcal{N}$. Luego, $\left\langle(y-t)-w, n_{0}\right\rangle_{B}=0 \mathrm{y}\left\langle n_{0}, n_{0}\right\rangle_{B}=0$ porque $n_{0} \in \mathcal{N}$. Por lo tanto,

$$
\langle y-(w+t), y-(w+t)\rangle_{B}=\left\langle(y-t)-s_{0},(y-t)-s_{0}\right\rangle_{B} \geq \min _{s \in \mathcal{S}_{+}}\langle y-(t+s), y-(t+s)\rangle_{B} .
$$

Finalmente, si calculamos el máximo sobre los vectores $t \in \mathcal{S}_{-}$, probamos la Ec. (5.3.3).

Recordemos además que la descomposición $R(C)=\mathcal{N} \dot{+} \mathcal{S}_{+} \dot{+} \mathcal{S}_{-}$de la Definición 5.3.1 no es necesariamente única. Sin embargo, el próximo resultado muestra que la definición de $B$-MMS es independiente de la descomposición seleccionada. Mas aún, la próxima proposición caracteriza a las $B$-MMS de la ecuación $C x=y$. En lo que resta de esta sección, $\mathcal{M}$ denotará al conjunto de vectores $B$-neutros de $\mathcal{K}$.

Proposición 5.3.3. Sean $C \in C R(\mathcal{H}, \mathcal{K})$ y $B \in L(\mathcal{K})^{s}$ tales que $R(C)$ es B-descomponible. Dado y $\in \mathcal{K}, u \in \mathcal{H}$ es una $B-M M S$ de $C x=y$ si y sólo si $u \in u_{0}+C^{-1}(\mathcal{M})$, siendo $u_{0} \in \mathcal{H}$ una solución de la ecuación normal $C^{*} B(C x-y)=0$.

Demostración. Supongamos que $u \in \mathcal{H}$ es una $B$-MMS de $C x=y$. Luego, por la Observación 5.3 .2 ,

$$
\langle y-C u, y-C u\rangle_{B}=\operatorname{máx}_{t \in \mathcal{S}_{-}} \min _{s \in \mathcal{S}_{+}}\langle y-(s+t), y-(s+t)\rangle_{B},
$$

donde $R(C)=\mathcal{N} \dot{+} \mathcal{S}_{+}+\mathcal{S}_{-}$es una descomposición como la de la Definición 5.3.1. Fijado un vector $t \in \mathcal{S}_{-}$, por el Lema 5.1.1, tenemos que el $\min _{s_{\in} \mathcal{S}_{+}}\langle(y-t)-s,(y-t)-s\rangle_{B}$ se alcanza en $s_{0}(t) \in \mathcal{S}_{+}$si y sólo si $\left\langle y-t-s_{0}(t), x\right\rangle_{B}=0$ para todo $x \in \mathcal{S}_{+}$. Entonces, $\left\langle y-s_{0}(t), x\right\rangle_{B}=$ 
$\left\langle y-t-s_{0}(t), x\right\rangle_{B}=0$ para todo $x \in \mathcal{S}_{+}$porque $\mathcal{S}_{-}$es $B$-ortogonal a $\mathcal{S}_{+}$. Por lo tanto, $s_{0}(t)=s_{0}$ es el único vector en $\mathcal{S}_{+}$que satisface

$$
\left\langle(y-t)-s_{0},(y-t)-s_{0}\right\rangle_{B}=\min _{s \in \mathcal{S}_{+}}\langle(y-t)-s,(y-t)-s\rangle_{B} .
$$

En conclusión, $\langle y-C u, y-C u\rangle_{B}=\operatorname{máx}_{t \in \mathcal{S}_{-}}\left\langle\left(y-s_{0}\right)-t,\left(y-s_{0}\right)-t\right\rangle_{B}$.

Analogamente, por la Observación 5.1.12, este máximo se alcanza en $t_{0} \in \mathcal{S}_{-}$si y sólo si $\left\langle y-s_{0}-t_{0}, t\right\rangle_{B}=0$ para todo $t \in \mathcal{S}_{-}$. Además, por la Observación 5.3.2, $\left\langle y-s_{0}-t_{0}, x\right\rangle_{B}=0$ para todo $x \in \mathcal{S}_{-}+\mathcal{N}$.

Sea $u_{0} \in \mathcal{H}$ tal que $C u_{0}=s_{0}+t_{0}$. Como $R(C)=\mathcal{N} \dot{+} \mathcal{S}_{+} \dot{+} \mathcal{S}_{-}$, es fácil ver que $\left\langle y-C u_{0}, z\right\rangle_{B}=$ 0 para todo $z \in R(C)$. Luego, $u_{0}$ es una solución de la ecuación normal $C^{*} B(C x-y)=0 \mathrm{y}$

$$
\langle y-C u, y-C u\rangle_{B}=\left\langle y-C u_{0}, y-C u_{0}\right\rangle_{B} .
$$

Como $\left\langle y-C u_{0}, z\right\rangle_{B}=0$ para todo $z \in R(C)$, tenemos que

$$
\begin{aligned}
\left\langle y-C u_{0}, y-C u_{0}\right\rangle_{B} & =\langle y-C u, y-C u\rangle_{B}= \\
& =\left\langle\left(y-C u_{0}\right)+\left(C u_{0}-C u\right),\left(y-C u_{0}\right)+\left(C u_{0}-C u\right)\right\rangle_{B}= \\
& =\left\langle y-C u_{0}, y-C u_{0}\right\rangle_{B}+\left\langle C u_{0}-C u, C u_{0}-C u\right\rangle_{B},
\end{aligned}
$$

o equivalentemente, $\left\langle C u_{0}-C u, C u_{0}-C u\right\rangle_{B}=0$. Por lo tanto, $u \in u_{0}+C^{-1}(\mathcal{M})$.

Recíprocamente, consideremos una solución $u_{0} \in \mathcal{H}$ de la ecuación normal $C^{*} B(C x-y)=0$ y sea $u \in u_{0}+C^{-1}(\mathcal{M})$. Notemos que $\langle C u-y, C u-y\rangle_{B}=\left\langle C u_{0}-y, C u_{0}-y\right\rangle_{B}$.

Supongamos que $R(C)=\mathcal{N} \dot{+} \mathcal{S}_{+} \dot{+} \mathcal{S}_{-}$es una descomposición como la de la Definición 5.3.1, y sean $x_{0} \in \mathcal{S}_{+}+\mathcal{N}$ y $t_{0} \in \mathcal{S}_{-}$tales que $C u_{0}=x_{0}+t_{0}$. Como $\left\langle y-C u_{0}, x\right\rangle_{B}=0$ para todo $x \in R(C)$, y $\mathcal{S}_{+}+\mathcal{N}$ es $B$-ortogonal a $\mathcal{S}_{-}$, es fácil ver que $\left\langle y-x_{0}-t, x\right\rangle_{B}=\left\langle y-x-t_{0}, t\right\rangle_{B}=0$ para todo $x \in \mathcal{S}_{+}+\mathcal{N}$ y $t \in \mathcal{S}_{-}$. Luego, dada $P$ la proyección ortogonal sobre $\mathcal{S}_{+}+\mathcal{N}$ y considerando la ecuación $P x=y-t_{0}$, por el Lema 5.1.1 tenemos que

$$
\begin{aligned}
\langle y-C u, y-C u\rangle_{B} & =\left\langle y-x_{0}-t_{0}, y-x_{0}-t_{0}\right\rangle_{B}=\min _{x \in \mathcal{H}}\left\langle y-t_{0}-P x, y-t_{0}-P x\right\rangle_{B}= \\
& =\min _{s \in \mathcal{S}_{+}+\mathcal{N}}\left\langle y-t_{0}-s, y-t_{0}-s\right\rangle_{B}=\min _{s \in \mathcal{S}_{+}}\left\langle y-t_{0}-s, y-t_{0}-s\right\rangle_{B}= \\
& =\operatorname{mín}_{s \in \mathcal{S}_{+}+\mathcal{N}} \operatorname{máx}_{t \in \mathcal{S}_{-}}\langle s+t-y, s+t-y\rangle_{B} .
\end{aligned}
$$

Corolario 5.3.4. Sean $C \in C R(\mathcal{H}, \mathcal{K})$ y $B \in L(\mathcal{K})^{\text {s }}$ tales que $R(C)$ es $B$-descomponible. Dado $y \in \mathcal{K}$, si $(B, R(C))$ es compatible entonces $u$ es una $B-M M S$ de $C x=y$ si y sólo si $C u \in$ $P_{B, R(C)} y+\mathcal{M}$.

Demostración. Supongamos que $(B, R(C))$ es compatible. Si $u$ es una $B$-MMS de $C x=y$ entonces $u=u_{0}+z$, siendo $u_{0}$ una solución de la ecuación normal $C^{*} B(C x-y)=0$ y $z \in$ $C^{-1}(\mathcal{M})$. Por el Corolario 5.1.15, $u_{0}=C^{\dagger} P_{B, R(C)} y+n$ con $n \in N(B C)=C^{-1}(\mathcal{N})$. Por lo tanto, $C u=C u_{0}+C z=P_{B, R(C)} y+C(n+z) \in P_{B, R(C)} y+\mathcal{M}$.

Recíprocamente, si $C u=P_{B, R(C)} y+z$ con $z \in \mathcal{M}$, luego $u=C^{\dagger} P_{B, R(C)} y+\left(C^{\dagger} z+P_{N(C)} u\right) \in$ $u_{y}+C^{-1}(\mathcal{M})$, donde $u_{y}$ es la solución minimal de la ecuación normal $C^{*} B(C x-y)=0$. Entonces, por la Proposición 5.3.3, $u$ es una $B$-MMS de la ecuación $C x=y$. 


\section{Bibliografía}

[1] A. Albert, Conditions for positive and nonnegative definiteness in terms of pseudoinverses, SIAM J. Appl. Math. 17, No. 2 (1969), 434-440.

[2] W. N. Anderson Jr., Shorted operators, SIAM J. Appl. Math. 20 (1971), 520-525.

[3] W. N. Anderson Jr. \& G. E. Trapp, Shorted operators II, SIAM J. Appl. Math. 28 (1975), $60-71$.

[4] T. Ando, Linear operators on Krein spaces, Hokkaido University, Sapporo, Japan, 1979.

[5] T. Ando, Generalized Schur complements, Linear Algebra Appl. 27 (1979), 173-186.

[6] J. Antezana, G. Corach \& D. Stojanoff, Spectral Shorted Operators, Integ. equ. oper. theory 55 (2006), 169-188.

[7] J. Antezana, G. Corach \& D. Stojanoff, Bilateral shorted operators and parallel sums, Linear Algebra Appl. 414, No. 2-3 (2006), 570-588.

[8] A. Ben-Israel \& T. N. E. Greville, Generalized inverse: Theory and Applications, SpringerVerlag, 2003.

[9] J. Bognár, Indefinite inner product spaces, Springer-Verlag, 1974.

[10] J. Bognár \& A. Krámli, Operators of the form $C^{*} C$ in indefinite inner product spaces, Acta Sci. Math. (Szeged) 29 (1968), 19-29.

[11] R. Bouldin, The product of operadors with closed range, Tohoku Math. J. 25 (1973), 359363.

[12] S. Boyd, L. Ghaoui, E. Feron \& V. Balakrishnan, Linear Matrix Inequalities in System and Control Theory, Studies in Applied Mathematics, SIAM, 1994.

[13] D. Carlson, E. Haynsworth \& T. Markham, A generalization of the Schur complement by means of the Moore-Penrose inverse, SIAM J. Appl. Math. 26, No. 1 (1974), 169-175.

[14] S. Chandrasekaran, M. Gu \& A. H. Sayed, A stable and efficient algorithm for the indefinite least squares problem, SIAM J. Matrix Anal. Appl. 20, No. 2 (1998), 354-362.

[15] J. S. Chipman, On least squares with insufficient observations, J. Amer. Statist. Assoc. 59 (1964), 1078-1111. 
[16] T. Constantinescu \& A. Gheondea, Representations of Hermitian kernels by means of Krein spaces, Publ. Res. Inst. Math. Sci. 33, No. 6 (1997), 917-951.

[17] J. B. Conway, A Course in Functional Analysis, Springer-Verlag, 1990.

[18] G. Corach \& A. Maestripieri, Weighted generalized inverses, oblique projections and least squares problems, Numer. Funct. Anal. Optim. 26, No. 6 (2005), 659-673.

[19] G. Corach, A. Maestripieri \& D. Stojanoff, Oblique projections and Schur complements, Acta Sci. Math. (Szeged) 67 (2001), 337-256.

[20] G. Corach, A. Maestripieri \& D. Stojanoff, A classification of projectors, Banach Center Publ., Institute of Mathematics, Polish Academy of Sciences, Warszawa, 67 (2004), 145160.

[21] G. Corach, A. Maestripieri \& D. Stojanoff, Projections in operator ranges, Proc. Amer. Math. Soc. 134, No. 3 (2006), 765-778.

[22] G. Corach, A. Maestripieri \& D. Stojanoff, Generalized Schur complements and oblique projections, Linear Algebra Appl. 341 (2002), 259-272.

[23] G. Corach, A. Maestripieri \& D. Stojanoff, Oblique projections and abstract splines, J. Approx. Theory 117 (2002), 189-206.

[24] G. Corach, A. Maestripieri \& D. Stojanoff, Generalized orthogonal projections and shorted operators, Margarita mathematica, Univ. La Rioja, España, 2001, 607-625.

[25] G. Corach, H. Porta \& L. Recht, The geometry of spaces of projections in $C^{*}$-algebras, Adv. Math. 101, No. 1 (1993), 59-77.

[26] F. Deutsch, The angle between subespacios in Hilbert spaces, "Aproximation theory, wavelets and applications" (S. P. Singh, editor), Kluwer, Netherlands, 1995, 107-130.

[27] J. Dixmier, Étude sur less variétés et les opérateurs de Julia avec quelques applications, Bull. Soc. Math. France 77 (1949), 11-101.

[28] R. G. Douglas, On majorization, factorization and range inclusion of operators in Hilbert space, Proc. Amer. Math. Soc. 17 (1966), 413-416.

[29] M. A. Dritschel \& J. Rovnyak, Operators on indefinite inner product spaces, Fields Institute Monographs No. 3, Amer. Math. Soc. Edited by Peter Lancaster, 1996, 141-232.

[30] M. A. Dritschel \& J. Rovnyak, Extension theorems for contraction operators on Krein spaces, Oper. Theory Adv. Appl. 47 (1990), 221-305.

[31] G. E. Dullerud \& F. Paganini, A Course in Robust Control Theory, Springer, 2000.

[32] L. Eldén, Perturbation theory for the least squares problem with linear equality contraints, SIAM J. Numer. Anal. 17, No. 3 (1980), 338-350.

[33] P. A. Fillmore \& J. P. Williams, On operator ranges, Advances in Math. 7 (1971), 254-281. 
[34] K. Friedrichs, On certain inequalities and characteristic value problems for analytic fuctions and for functions of two variables, Trans. Amer. Math. Soc. 41 (1937), 321-364.

[35] Yu. P. Ginzburg \& I. S. Iokhvidov, Investigation by geometry of infinite-dimensional spaces with a bilinear metric (Russ.), Uspekhi Mat. Nauk., 1962, 17(4), 3-56.

[36] J. I. Giribet, A. Maestripieri \& F. Martínez Pería, Shorting selfadjoint operators in Hilbert spaces, Linear Algebra Appl. 428 (2008), 1899-1911.

[37] P. R. Halmos, Introduction to Hilbert spaces and the theory of spectral multiplicity, Chelsea Publishing Company, New York, 1972.

[38] S. Hassi \& K. Nordström, On projections in a space with an indefinite metric, Linear Algebra Appl. 208/209 (1994), 401-417.

[39] B. Hassibi, A. H. Sayed \& T. Kailath, $\mathcal{H}^{\infty}$ Optimality Criteria for LMS and Backpropagation, Advances in Neural Information Processing Systems, Vol. 6 (1994), 351-359.

[40] B. Hassibi, A. H. Sayed \& T. Kailath, Linear Estimation in Krein Spaces - Part I: Theory, IEEE Transactions on Automatic Control 41, No. 1 (1996), 18-33.

[41] B. Hassibi, A. H. Sayed \& T. Kailath, Linear Estimation in Krein Spaces - Part II: Application, IEEE Transactions on Automatic Control 41, No. 1 (1996), 33-49.

[42] B. Hassibi, A. H. Sayed \& T. Kailath, Indefinite-Quadratic Estimation and Control. A Unified Approach to $\mathcal{H}^{2}$ and $\mathcal{H}^{\infty}$ Theories, SIAM. Studies in Applied and Numerical Mathematics, 1999.

[43] I. S. Iokhvidov \& T. Ya. Azizov, Linear Operators in spaces with an indefinite metric, John Wiley and sons, 1989.

[44] I. S. Iokhvidov \& M. G. Krein, Spectral theory of operators in spaces with an indefinite metric I, (Russ.) Trudy Moskov, Mat. Obshch. 5 (1956), 367-432; Amer. Math. Soc. Translations (2), No. 13 (1960), 105-175.

[45] I. S. Iokhvidov \& M. G. Krein, Spectral theory of operators in spaces with an indefinite metric II, (Russ.) Trudy Moskov, Mat. Obshch. 8 (1959), 413-496; Amer. Math. Soc. Translations (2), No. 34 (1963), 283-374.

[46] I. S. Iokhvidov, M. G. Krein \& H. Langer, Introduction to the Spectral Theory of Operators in Spaces with an Indefinite Metric, Akademie-Verlag, Berlin, 1982.

[47] S. Izumino, The product of operadors with closed range and an extension of the reverse order law, Tohoku Math. J. 34 (1982), 43-52.

[48] R. Kalman, A new approach to linear filtering and prediction problems, Journal of Basic Eng., ASME, Series D, Vol. 82 (1960), 35-45.

[49] T. Kato, Perturbation theory for linear operators (reprint of the 1980 edition), SpringerVerlag, Berlin, 1995. 
[50] A. N. Kolmogorov, Stationary sequences in Hilbert spaces, Bull. Math. Univ. Moscow 2 No. 6 (1941).

[51] M. G. Krein, Introduction to the geometry of indefinite $J$-spaces and the theory of operators in those spaces (Russ.), "Second Summer Mathematical School I", 15-92, Kiev, 1965.

[52] M. G. Krein, The theory of self-adjoint extensions of semibounded Hermitian operators and its applications, Mat. Sb. (N. S.) 20 (62) (1947), 431-495.

[53] A. Maestripieri \& F. Martínez Pería, Decomposition of Selfadjoint Projections in Krein Spaces, Acta Sci. Math. 72 (2006), 611-638.

[54] P. Massey \& D. Stojanoff, Generalized Schur Complements and P-Complementable Operators, Linear Algebra Appl. 393 (2004), 299-318.

[55] E. H. Moore, On the reciprocal of the general algebraic matrix, Bull. Amer. Math. Soc. 26 (1920), 394-395.

[56] M. Z. Nashed, Inner, outer, and generalized inverses in Banach and Hilbert spaces, Numer. Funct. Anal. Optim. 9, No. 3-4 (1987), 261-325.

[57] M. Z. Nashed \& G. F. Votruba, A unified operator theory of generalized inverses, Generalized inverses and applications (Proc. Sem., Math. Res. Center, Univ. Wisconsin, Madison, Wis., 1973), 1-109. Publ. Math. Res. Center Univ. Wisconsin, No. 32, Academic Press, New York, 1976.

[58] M. Z. Nashed \& G. Wahba, Generalized inverses in reproducing kernel spaces: an approach to regularization of linear operator equations, SIAM J. Math. Anal. 5 (1974), 974-987.

[59] E. L. Pekarev, Shorts of operators and some extremal problems, Acta Sci. Math. (Szeged) 56 (1992), 147-163.

[60] E. L. Pekarev \& Ju. L. Smul'jan, parallel addition and parallel subtraction of operators, (Russian) Izv. AN USSR 49 (1976), 366-387.

[61] R. Penrose, A generalized inverse for matrices, Proc. Cambridge Philos. Soc. 51 (1955), 406-413.

[62] L. S. Pontryagin, Hermitian operator in spaces with indefinite metric (Russ.), Izvestiya Akad. Nauk USSR, Ser. Matem. 8 (1944), 243-280.

[63] C. R. Rao \& S. K. Mitra, Generalized inverse of a matrix and its applications, Wiley and Sons, New York, 1971.

[64] C. R. Rao \& S. K. Mitra, Generalized inverse of a matrix and its applications. Proceedings of the Sixth Berkeley Symposium on Mathematical Statistics and Probability (Univ. California, Berkeley, California, 1970/1971), Vol. I: Theory of statistics, 601-620. Univ. California Press, Berkeley, California, 1972.

[65] J. Rovnyak, Methods on Krein space operator theory, Interpolation theory, systems theory and related topics (Tel Aviv/Rehovot, 1999), Oper. Theory Adv. Appl., 134 (2002), 31-66. 
[66] A. H. Sayed, B. Hassibi \& T. Kailath, Fundamental Inertia Conditions for the Minimization of Quadratic Forms in Indefinite Metric Spaces, Oper. Theory: Adv. Appl., Birkhauser, Cambridge, 1996.

[67] A. H. Sayed, B. Hassibi \& T. Kailath, Inertia properties of indefinite quadratic forms, IEEE Signal Processing Letters 3, No. 2 (1996), 57-59.

[68] I. Schur, Potenzreihen im Innern des Einheitskreises, J. Reine Agnew. Math. 147 (1917), 205-232.

[69] Ju. L. Smul'jan, A Hellinger operador integral, (Russian) Mat. Sb. (N.S.) 49 (91) 1959 381-430; (English) Amer. Math. Soc. Translations 22 (1962), 289-337.

[70] X. Sheng \& G. Chen, The generalized weighted Moore-Penrose inverse, J. Appl. Math. \& Computing, 25 (2007), 407-413.

[71] N. Wiener, Extrapolation, interpolation and smoothing of stationary time series with engineering applications, Technology Press and Wiley, New York, NY, 1949.

[72] G. Zames, Feedback and optimal sensitivity: Model reference transformations, multiplicative seminorms, and approximate inverses, IEEE Trans. on Automatic Control 26 (1981), 301-320. 